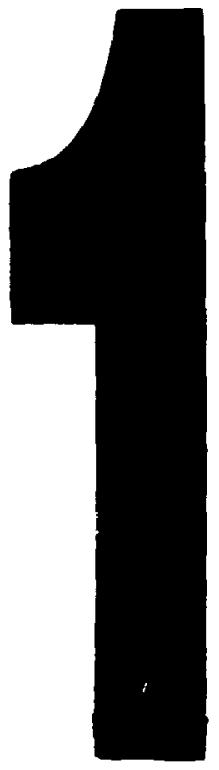

PM-1 31/2"x4" PHOTOGRAPHIC MICROCOPY TARGET NBS 1010a ANSI/ISO \#2 EQUIVALENT

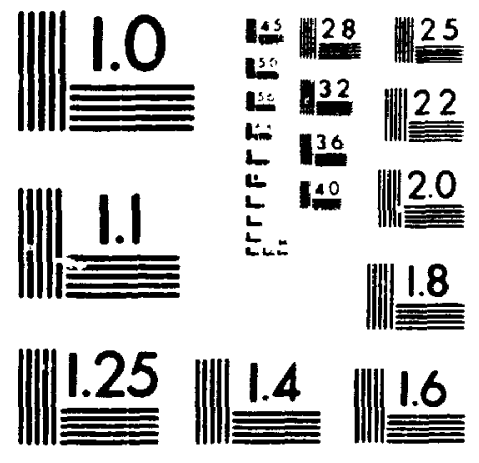

PRECISIONSM RESOLUTION TARGETS 
Acquisitions and

Bibliographic Serv ce; Branch

395 Wellington Sireet

Ottawa Ontario

KIA ONA

\section{Bibliothèque nationale}

du Canada

Direction des acquisitions et

des services bibilographiques

395, rue Wellington

Ottawa (Ontario)
NOTICE
AVIS
The quality of this microform is heavily dependent upon the quality of the original thesis submitted for microfilming. Every effort has been made to ensure the highest quality of reproduction possible.

If pages are missing, contact the university which granted the degree.

Some pages may have indistinct print especially if the original pages were typed with a poor typewriter ribbon or if the university sent us an inferior photocopy.

Reproduction in full or in part of this microform is governed by the Canadian Copyright Act, R.S.C. 1970, c. C-30, and subsequent amendments.
La qualité de cette microforme dépend grandement de la qualité de la thèse soumise au microfilmage. Nous avons tout fait pour assurer une qualité supérieure de reproduction.

S'il manque des pages, veuillez communiquer avec l'université qui a conféré le grade.

La qualité d'impression de certaines pages peut laisser à désirer, surtout si les pages originales ont été dactylographiées à l'aide d'un ruban usé ou si l'université nous a fait parvenir une photocopie de qualité inférieure.

La reproduction, même partielle, de cette microforme est soumise à la Loi canadienne sur le droit d'auteur, SRC 1970, c. C-30, et ses amendements subséquents. 


\title{
AN INVESTIGATION OF THE EFFECTS OF ROUGHNESS ON THE PERFORMANCE OF A TURBINE CASCADE
}

by

\author{
Peter John Serjak \\ B.Eng. (Mechanical Engineering)
}

\begin{abstract}
A thesis submitted to
the Faculty of Graduate Studies and Research

in partial fulfilment of the requirements

for the degree of
\end{abstract}

\section{MASTER OF ENGINEERING \\ in}

Mechanical Engineering

Ottawa-Carleton Institute for Mechanical and Aerospace Engineering

Department of Mechanical and Aerospace Engineering

Carleton University

Ottawa, Ontario, Canada

، Til, 1995

Copyright @ P. J. Serjak 1995 
National Library

of Canada

Acquisitions and

Bibliographic Services Branch

395 Wellington Street

Ottawa. Ontano

KIA ONA
Bibliotheque nationale

du Canada

Direction des acquisitions et des services bibliographiques

395. ne Wellington

Ottawa (Ontano)
THE AUTHOR HAS GRANTED AN IRREVOCABLE NON-EXCLUSIVE LICENCE ALLOWING THE NATIONAL LIBRARY OF CANADA TO REPRODUCE, LOAN, DISTRIBUTE OR SELL COPIES OF HIS/HER THESIS BY ANY MEANS AND IN ANY FORM OR FORMAT, MAKING THIS THESIS AVAILABLE TO INTERESTED PERSONS.
L'AUTEUR A ACCORDE UNE LICENCE IRREVOCABLE ET NON EXCLUSIVE PERMETTANT A LA BIBLIOTHEQUE NATIONALE DU CANADA DE REPRODUIRE, PRETER, DISTRIBUER OU VENDRE DES COPIES DE SA THESE DE QUELQUE MANIERE ET SOUS QUELQUE FORME QUE CE SOIT POUR METTRE DES EXEMPLAIRES DE CETTE THESE A LA DISPOSITION DES PERSONNE INTERESSEES.
THE AUTHOR RETAINS OWNERSHIP OF THE COPYRIGHT IN HIS/HER THESIS. NEITHER THE THESIS NOR SUBSTANTIAL EXTRACTS FROM IT MAY BE PRINTED OR OTHERWISE REPRODUCED WITHOUT HIS/HER PERMISSION.
L'AUTEUR CONSERVE LA PROPRIETE DU DROIT D'AUTEUR QUI PROTEGE SA THESE. NI LA THESE NI DES EXTRAITS SUBST ANTIELS DE CELLECI NE DOIVENT ETRE IMPRIMES OU AUTREMENT REPRODUITS SANS SON AUTORISATION.

ISBN $\quad 0-612-03034-2$ 
Name Pete. I T- jek

Dissertation Abstracts International is arranged by broad, general subject categories Please select the one subject which most nearly describes the content of your dissertation Enter the corresponding four-digit code in the spaces provided

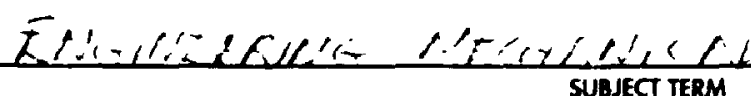

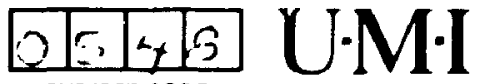

SUBJECT CODE

\section{Subject Categories}

\section{THE HUMANITIES AND SOCIAL SCIENCFS}

\section{COMMUNICATONS AND THE ARTS}

Architecture

Ant Hision

Cinemo

Dance

Informotion Science

Journalism

Librory Science

Mass Communications

Music

Speech Communication

Theoter

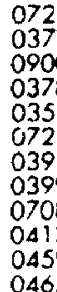

\section{coucation}

Genero

Adult and Cantinuing

Agricultural

Bilingual and Mulheultural

Business

Community College

Curriculum and instruction

Early Childhood

Elemeritary

Guidonce and Counseling

Heolith

Higher

Home Economic

Industriol

Language and Literoture

Mathernatics

Music

Philosophy of

Physi ol

0517

0273

0282

0278

0280
Psychology

Reoding

Sciences

Secondary

Socidi Sciences

Sociology of

Special

Teacher Training

Technology

Tests and Meosurements

Vocational

LANGUAGE, LITERATURE ANO

LINGUISTICS

0515

0514

0516

0688
0275

0275
0727

0727
0518

0524

0277

0519

0680

0745

0520

0521
0279

0522

0523
Lonquage

Genero

Ancient

Moder

Literature

General

Comparative

Medievol

Modern

Americon

Americon

Canodion (English)

Conadian (French)

Englis?

Germanic

Latin Americon

Middie Eastern

Romance

Slavic and East Europeon

0679

028

0290

0291

0401

0294

0297

0298

0316

059

305

0352

0355

(1)

$03 ! 1$

0312

0315
0313

0314
PHILOSOPHY, RELIGION AND

THEOLOGY

Philosophy

Religion

Generd

Bibliral Studies

Clergy

History of

Philosophy of

Theology

SOCIAL SCIENCES

American Studies

Anthropology

Archoeology

Culpural

Physical Adminto

Get stol

Accounting

Eanking

Moriagement

Norkpeing

Canodian Studies

Economics

Genera!

Agricuterat

Commerce Busines:

funenes

History

ator

Theory

Folklore

Geography

Geronitongy

History

Generd
0422

0318

0321

0320

0322

0469

0323

0324

0326

0327

C 310

0272

0770

0338

0385

2501

0503

0505

0508

0509

0510

0358

0351

0578

\section{THE SCIENCES AND ENGINEERING}

\section{DIOLOGICAL SCIENCES}

General

Agronomy

Animal Culture and

Nutrition

Animal Pathulogy

Food Science and

Technology

Forestry and Wildlite

Plant Culture

Piant Pathology

Plant Physiology

Ronge Management

ology

Anotomy

Giostolistics

Botony

Cell

Ecology

Entomology

Genefics

Limnology

Nitcrobiolog

Molecular

Neuroscrence

Physiology

Radiation

Veterinory Science

Zoology

Biophysics

Generol

MTH sciences

Biogeochemisiny

Geochemistry
Geodesy

0473

0285

0475

0476

0359

0478

0479

0480

0817

0777

0746

0306

$028 \%$

0308

0309

0379

0329

0353

0369

0410

0410
0307

0307
$03 ! 7$

0416

0433

0821

0778

0472

0780

0760

0425

Geophysics

Aydrology

SCIENCES

Poleobotony

Palececology

Poleontology

Paleozoology

Physical Geography

Fhysical Oceonography

MEALTH AND ENVIRONMENTAL

Envirormental Sciences

Health Sciences

Generd

Audiology

Che: $r$ therapy

Denusiry

Hospital Manogement

Human Development

Immunology

Medirine and Surgery

Menta! Healt:

Nursing

Nutrition 0570

Obstetrics and Gynecology 0380

Occupalional Health and

Theropy

Ophthalmology

Pathology 
The undersigned recommend to the Faculty of Graduate Studies and Research acceptance of the thesis

\section{AN INVESTIGATION OF THE EFFECTS OF ROLGHNESS ON THE PERFORMANCE OF A TURBINE CASCADE}

submitted by

Peter John Serjak, B.Eng.

in partial fulfilment of the requirements for the degree of Master of Engineermg

Profensor R.J. Kind, Thess Supersicor

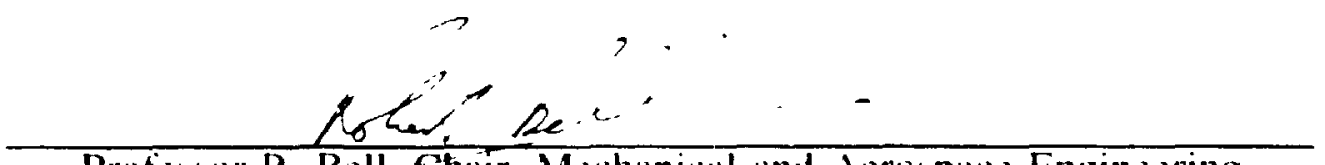

Professor R. Bẹll Chair, Mechanical and Aerospace Engineering

Carleton Liniversity

April 17. 1995 


\begin{abstract}
In-service damage to compressor or turbine blades is a common cause of performance deterioration in gas-turbines. One type of damage is blade surface roughening due to fouling, erosion, or corrosion. The present research examines the effect of surface rougriening on the aerodynamics of turbine blades. A combination of experimental measurements and computations were used during the investigation.
\end{abstract}

Experimental measurements were conducted using a low-speed, large-scale cascade. Computations were made using a viscous/inviscid interaction approach. Experimental and predicted static pressure distributions, profile loss and flow deflection results were compared for design and off-design conditions with various configurations of surface roughness. Random!y distributed sand grains adhered to the surface of the blades were used to simulate surface icughening. The roughness height, the width of the roughness band, and the location of the band were varied.

The surface roughness significantly increased profile losses when present on the suction surface especially near the leading edge. It has only minor effects when present on the pressure surface and has little effect on flow turning in either case. The computational method gives good predictions of the effects of roughness on the pressure distribution, profile losses and flow turning. 


\section{Acknowledgements}

I would like to extend my most sincere appr sciation to my thesis supervisor. Professor R. J. Kind, for his continued interest, encouragement and expertise provided during this research.

The financial support from GasTOPS Ltd., Ottawa, Canada and the Government of Ontario under the University Research Incentives Fund is gratefully acknowledged.

To all my colleagues, too many to name, thanks for everything. My work could not have been completed without the assistance of two colleagues in particular, Michael W. Benner and Stefano F. De Cecco, whose knowledge and guidance were instrumental throughout. In addition, a special thanks to my parents and friend Angela M. Pears for their support and encouragement throughout.

I would also like to extend my thanks to the employees of Engineering Technical Centre (Mechanical), in particular to Stephen J. Szick who always took the time to lerd a helping hand. Finally, to the staff of the Department of Mechanical and Aerospace Engineering office, thanks sor everything. 


\section{Table of Contents}

Abstract

page

Acknowledgements

i

Table of Contents

ii

List of Figures

iii

List of Tables

viii

xiii

Nomenclature

CHAPTER 1 INTRODUCTION

2.1 Cascade Terminology 3

2.2 Losses

2.3 Flow Over Rough Surfaces

3.1 Wind Tunnel

3.1.1 Cascade Test Section

3.1.2 Cascade Blades

3.2 Data Acquisition Equipment and Procedures

3.2.1 Data Acquisition Equipment

3.2.2 Three-Hole Pressure Probes

3.2.2.1 Calibration and Measurements 
3.2.2.2 Accuracy of the Three-Hole Probe 29

3.3 Measurement of Cascade Performance 30

3.3.1 Ope rating Point 30

3.3.2 Inlet Measurements 30

3.3.3 Outlet Flow Uniformity and Periodicity 33

3.3.4 Flow Two-Dimensionality and Axial Velocity Ratio 34

3.3.5 Data Reduction 36

3.3.6 Blade Static Pressure Measurements 39

3.3.7 Boundary Layer Transition Measurements 40

3.4 Roughness Selection and Application 40

3.4.1 Roughness Selection Procedure 40

3.4.2 Roughness Application Equipment $\quad 42$

3.4.3 Roughness Application Procedure 43

3.4.4 Examination of the Roughness Distribution 44

CHAPTER 4 COMPUTATIONAL ANALYSIS

4.1 Outline of the Computational Method 46

4.2 Application of the Computational Method 50

4.2.1 Modified Blade Geometry 50

4.2.2 Kutta Condition 52

4.2.3 Computational Procedures and Practices 53

4.2.3.1 Cascade Configuration 53

4.2.3.2 Boundary Layer Transition 53

4.2.3.3 Surface-Source Distribution 56

4.3 Post-Processing of Computational Results 57

4.3.1 Pressure Coefficients 57

4.3.2 Loss Prediction Schemes $\quad 58$

$\begin{array}{lll}4.4 & \text { Potential Sources of Error } & 60\end{array}$ 
5.1 Experimental Results 62

5.1.1 Blade Static Pressure Distribution 66

$\begin{array}{lll}\text { 5.1.1.1 Rough-Surface Blades } & 69\end{array}$

5.1.1.1.1 Design Incidence $\quad 69$

5.1.1.1.2 Off-Design Incidence 70

5.1.2 Total Pressure Loss and Flow Deviation 73

$\begin{array}{ll}\text { 5.1.2.1 Smooth Blades } & 73\end{array}$

5.1.2.2 Effect of Roughness 76

5.1.2.2.1 Design Incidence 76

5.1.2.2.2 Off-Design Incidence $\quad 79$

5.2 Computational Results 83

5.2.1 Validation of the Inviscid Solution 83

5.2.2 Computational Results and Comparison with Experimental 83

5.2.2.1 Static Pressure Distribution 83

5.2.2.1.1 Smooth Blades 83

5.2.2.1.2 Rough-Surface Blades 87

5.2.2.1.2.1 Design Incidence 87

5.2.2.1.2.2 Off-Design Incidence 88

5.2.2.2 Pressure Loss and Flow Deviation 91

5.2.2.2.1 Design Incidence 91

5.2.2.2.2 Off-Design Incidence 93

CHAPTER 6 CONCLUSIONS AND RECOMMENDATIONS 99

6.1 Conclusions 99

6.2 Recommendations for Further Study 100

$\begin{array}{ll}\text { REFERENCES } & 102\end{array}$ 
APPENDICES

105

Appendix A Blade and Cascade Geometry

Appendix B Static Pressure Blade Geometry

105

Appendix C Calibration Data

107

Appendix D Control Surface

109

Appendix E Spacing Parameter Calculations

116

Appendix F Three-Hole Probe Drawings

118

Appendix G Sample Input File of the Modified Blade Geometry

120

Appendix $\mathrm{H}$ Derivations of the Computational Loss Coefficient

121

124

vii 


\section{List of Figures}

Figure 2.1 Cascade geometry and nomenclature. 4

Figure 2.2 Static pressure distribution for various roughness heights -

reproduced from Barnmert and Sandstede (1980).

Figure 2.3 Velocity distribution along profile $(\mathrm{k} / \mathrm{c}=0.00331)$ reproduced from Bammert and Sandstede (1980).

Figure 2.4 Shape factor on the suction surface - reproduced from Bammert and Sandstede (1980).

Figure 2.5 Momentum thickness on the suction surface - reproduced from Bammert and Sandstede (1980).

Figure 2.6 Calculated momentum thickness on the suction surface.

Measured points are represented by the open symbols reproduced from Bammert and Sandstede (1980).

Figure 2.7 Efficiency for smooth and rough blades (where: $U$ = mean blade speed, Co $=$ Overall isentropic velocity total-total) reproduced from Boynton et al. (1992).

Figure 2.8 Smooth and rough blade predicted and measured efficiency (where: $U_{m}=$ mean wheel speed, $V_{\text {IDEAL }}$ - ideal isentropic velocity) - reproduced from Bcyle (1993).

Figure 2.9 Efficiency-pressure ratio characteristics at design speed reproduced from Suder et al. (1994).

Figure 2.10 Pressure rise characteristics for the rough coatings at design speed - reproduced from Suder et al. (1994).

Figure 2.11 Comparison between predicted and measured pressure rise versus massflow characteristics at $70 \%$ span for the smooth coated blade - reproduced from Suder et al. (1994).

Figure 2.12 Comparison between predicted and measured pressure rise versus massflow characteristics at $70 \%$ span for the rough coated blade - reproduced from Suder et al. (1994).

Figure 3.1 Schematic of wind tunnel - reproduced from Rodger (1992). 
Figure 3.2 Variable incidence cascade test section - reproduced from Sjolander et al. (1993).

Figure 3.3 Cascade geometry and measuring plane nomenclature. 21

Figure 3.4 Data acquisition system set up for three-hole pressure probe 23 measurements.

Figure 3.5 Three-nole pressure probe geometry. 26

$\begin{array}{lll}\text { Figure 3.6 Data acquisition setup for calibration apparatus. } & 27\end{array}$

$\begin{array}{lll}\text { Figure 3.7 } & \text { Test section control surfaces. } & 31\end{array}$

Figure 3.8 Typical inlet flow angle distribution at design incidence. 32

Figure 3.9 Typical outlet flow angle distribution at design incidence. 32

Figure 3.10 Typical outlet total-pressure-loss coefficient distribution at 33 design incidence.

Figure 3.11 Schematic top view of the cascade and endplates presented with the interpolation scheme used to determine the axial velocity ratio.

Figure 3.12 Data acquisition setup for static blade measurements. 38

Figure 3.13 Relative size of sand grains. 41

Figure 3.14 Schematic of roughness application apparatus. 43

Figure 4.1 Schematic illustration of the inviscid computational model. 47

Figure 4.2 Schematic illustration of a finite array of profiles. 48

Figure 4.3 Original and modified blade profile. 51

Figure 4.4 Schematic illustration of the vorticity distribution at the trailing 52 edge for the third form of the Kutta condition.

Figure 4.5 Input parameters for computations on the present cascade. 54

Figure 4.6 Typical pressure distribution determined both experimentally 55 and computationally.

Figure 4.7 Trailing edge loss (energy) coefficient correlated against the ratio of trailing edge thickness to throat opening - reproduced from Kacker and Okapuu (1982).

Figure 4.8 Schematic representation of the trailing edge with the additional source strength scheme. 
Figure 5.1 Locations and roughness band widths on the blade profile.

Figure 5.2 Midspan blade static pressure distributions at design incidence; comparison with Whitehouse (1993).

Figure 5.3 Midspan blade static pressure distributions with and with roughness applied to either the pressure and suction surfaces at design incidence.

Figure 5.4 Midspan static pressure distrikution with and without roughness applied to the suction surface at $+15^{\circ}$ incidence.

Figure 5.5 Midspan blade static pressure distribution with and without roughness applied to the suction and pressure surface at $+10^{\circ}$ incidence.

Figure 5.6 Midspan blade static pressure distribution with various roughness sizes applied to the pressure surface at $-10^{\circ}$ incidence.

Figure 5.7 Total-pressure-loss coefficient versus incidence for smooth blades; comparison with Whitehouse (1993) and Abbott (1993).

Figure 5.8 Flow deviation angle versus incidence for smooth blades; comparison with Whitehouse (1993) and Abbott (1993).

Figure 5.9 Total-pressure-loss coefficient versus roughness height, for design incidence; comparison with Abbott (1993).

Figure 5.10 Flow deviation angle versus roughness height, for design incidence; comparison with Abbott (1993).

Figure 5.11 Total-pressure-loss coefficient versus roughness height, for design incidence.

Figure 5.12 Flow deviation angle versus roughness height, for design incidence.

Figure 5.13 Total-pressure-loss coefficient versus incidence for various roughness band widths (with $k / c=0.00445, L E=0.10 \mathrm{x} / \mathrm{c}$ ).

Figure 5.14 Total-pressure-loss coefficient versus incidence for various roughness configurations applied to the suction and pressure surface $(\mathrm{LE}=0.25 \mathrm{x} / \mathrm{c})$.

Figure 5.15 Flow deviation angle versus incidence for various roughness band widths (with $k / c=0.00445, L E=0.10 \mathrm{x} / \mathrm{c}$ ). 
Figure 5.16 Flow deviation angle versus incidence for various roughness configurations applied to the suction and pressure surrace (LE $=0.25 \times / c)$.

Figure 5.17 Pressure distributions and blade profile for the Gostellow cusped blade cascade; comparison with inviscid solution.

Figure 5.18 Pressure distributions and blade profile for the CC3 blade cascade; comparison with the experimental results, and the inviscid and viscous/inviscid predictions.

Figure 5.19 Predicted pressure distribution with and without roughness applied to the suction and pressure surface at design incidence.

Figure 5.20 Predicted pressure distributions at off-design incidence angle; comparison with experimental data.

Figure 5.21 Predicted pressure distribution with and without roughness applied to the pressure and suction surfaces at $+10^{\circ}$ incidence.

Figure 5.22 Predicted pressure distribution with and without roughness applied to the pressure surface at $-10^{\circ}$ incidence.

Figure 5.23 Predicted and measured total-pressure-loss coefficient versus roughness height, for design incidence.

Figure 5.24 Predicted and measured flow deviation angle versus roughness height, for design incidence.

Figure 5.25 Predicted and measured total-pressure-loss coefficient versus incidence for various roughness locations on the suction surface (with $\mathrm{k} / \mathrm{c}=0.00445, \Delta \mathrm{s} / \mathrm{c}=0.24$ ).

Figure 5.26 Predicted and measured total-pressure-loss roefficient versus incidence for medium and large size roughness on the pressure surface (with $\lambda=0.25 \mathrm{PS}, \Delta \mathrm{s} / \mathrm{c}=0.71$ ).

Figure 5.27 Predicted and measured flow deviation angle versus incidence for various roughness locations on the suction surface (with $\mathrm{k} / \mathrm{c}$ $=0.00445, \Delta \mathrm{s} / \mathrm{c}=0.24$ ).

Figure 5.28 Predicted and measured flow deviation angle versus incidence for medium and large size roughness applicd on the pressure surface (with $\mathrm{x} / \mathrm{c}=0.25 \mathrm{PS}, \Delta \mathrm{sic}=0.71$ ).

Figure 5.29 Predicted and measured total-pressure-loss coefficient versus incidence for large and small roughness sizes and for $0.24 \Delta \mathrm{s} / \mathrm{c}$ and $0.47 \Delta s / c$ roughness band widths $(\mathrm{LE}=0.25 \mathrm{x} / \mathrm{c}$ ). 
Figure 5.30 Predicted and measured flow deviation angle versus incidence for large and small roughness sizes and for $0.24 \Delta \mathrm{s} / \mathrm{c}$ and 0.47 $\Delta s / c$ roughness band widths (LE $=0.25 \mathrm{x} / \mathrm{c}$ ).

Figure B.1 Static tap location and orientation nomenclature for the instrumented blade.

Figure C.1 Three-hole probe port numbering.

Figure C.2 Variation of $K_{1}$ with yaw angle for the outlet probe.

Figure C.3 Variation of $\mathbf{K}_{12}$ with yaw angle for the outlet probe.

Figure C.4 Variation of $K_{13}$ with yaw angle for the outlet probe.

112

Figure C.5 Variation of $\mathbf{K}_{23}$ with yaw angle for the outlet probe.

Figure C.6 Variation of $\mathrm{K}_{62}$ with yaw angie for the outlet probe.

Figure C.7 Variation of $\mathrm{K}_{63}$ with yaw angle for the outiet probe.

113

Figure C.8 Rusemount (Serial \#567) pressure transducer calibration curve.

Figure C.9 Baratron (Serial \#79508-2) pressure transducer calibration curve.

Figure D.1 Endplate nomenclature and settings - reproduced from Whitehouse (1993).

Figure D.2 Control surface locations and labelling - reproduced from Rodger (1992).

Figure E.1 Approximate locations of the eight areas investigated for suction- and pressure- surface roughness samples.

Figure F.1 Three-hole probes.

Figure H.2 Cross section of blade wake far downstream. 


\section{List of Tables}

page

Table 2.1 Smooth and rough blade averages at 104\% RPL equivalent conditions - reproduced from Boynton et al. (1992).

Table 3.1 Sand grain sizes.

Table 5.1 Roughness configurations, measured and predicted data (experimental data reproduced from Abbott (1993)).

Table 5.1b Roughness configurations, measured and predicted data (all data shown are from the present study).

Table A.1 Summary of geometric and aerodynamic parameters of the cascade blades.

Table A.2 Blade profile coordinates.

$\begin{array}{lll}\text { Table B.1 Static pressure tap coordinates and orientation. } & 108\end{array}$

Table D.1 Control surface and endplate settings. 116

Table E.1 Spacing parameter calculation data. 


\section{Nomenclature}

\section{English Symbols}

$A_{F} \quad=$ total average frontal area of particles

$A_{T}=$ total area

c - blade chord length

$\mathrm{c}_{\mathrm{f}} \quad=$ skin friction coefficient

$c_{x}=$ blade axial chord length

$\mathrm{Cp}=\frac{P_{s}-P_{1}}{\frac{1}{2} \rho V_{1}^{2}}=$ static pressure coefficient referenced to cascade inlet

$\mathrm{C}_{\mathrm{pmes}}=\frac{P_{s}-P_{\text {ref }}}{\frac{1}{2} \rho V_{\text {ref }}^{2}}=$ static pressure coefficient referenced far upstream

$\mathrm{CP}_{\mathrm{P}}=\frac{P_{01}-P_{0_{\text {nf }}}}{\frac{1}{2} \rho V_{\text {nf }}^{2}}=$ total-pressure-loss coefficient

h = blade span

$\mathrm{H}=\frac{\delta^{*}}{\theta}-$ boundary layer shape factor

i $\quad=\alpha_{1}-\beta_{1}=$ incidence angle

k $\quad$ - nominal grain size

$\dot{m}=$ mass flow rate

M = Mach number

$\mathrm{N} \quad$ - number of junction points

P $\quad$ - static pressure 
$P_{S} \quad$ - blade surface pressure

$q \quad-\frac{1}{2} \rho V^{2}=$ dynamic pressure

Q - point source strength

$\operatorname{Re} \quad=\frac{\rho V_{\text {ref }} c}{\mu}=$ Reynolds number based on blade chord

s $\quad$ - streamwise coordinate

S = blade spacing

$\mathrm{t} \quad=$ trailing edge thickness

$\mathrm{U}_{\mathrm{E}}$ - local inviscid velocity

$\mathrm{V}=$ resultant velocity

We = leading-edge wedge angle

$x, y=$ coordinates in axial and tangential direction

$\mathrm{Y}_{\mathrm{m}}=$ total-pressure-loss coefficient (profile)

\section{Greek Symbols}

$$
\begin{array}{ll}
\alpha & =\text { flow angle } \\
\beta & =\text { blade metal angle } \\
\gamma & =\text { blade stagger angle } \\
\gamma_{N} & =\text { vorticity } \\
\Delta & =\text { change } \\
\Delta \phi^{2} & =1-\frac{V_{2}^{2}}{V_{2 \text { sen }}^{2}}=\text { kinetic-energy-loss coefficient } \\
\delta & =\alpha_{2}-\beta_{2}=\text { flow deviation angle }
\end{array}
$$


$\delta^{*} \quad$ - boundary layer displacement thickness

$\eta \quad$ - efficiency

$\theta \quad$ - boundary layer momentum thickness

$\lambda \quad-\frac{A_{T}}{A_{F}}=$ spacing parameter

$\mu \quad$ - dynamic viscosity

$v \quad$ - kinematic viscosity

$\rho \quad$ = density

$\sigma \quad$ - blade passage throat opening

\section{Subscripts}

$$
\begin{aligned}
& \text { des } \quad \text { - design incidence } \\
& \text { far } \quad \text { - far downstream of the cascade } \\
& \text { isen } \quad \text { - isentropic } \\
& \text { le } \quad \text { - leading edge } \\
& \text { m } \quad \text { - mixed-out losses } \\
& \text { meas } \quad \text { - measured } \\
& \text { o } \quad \text { - stagnation (total) quantity } \\
& \text { PS } \quad \text { - pressure surface } \\
& \text { ref } \quad \text { - reference plane } \\
& \text { SS } \quad \text { - suction surface } \\
& \text { te } \quad \text { - trailing edge } \\
& \text { wake } \quad \text { - in the blade wake } \\
& 1,2 \quad=\text { cascade inlet and outlet }
\end{aligned}
$$




\section{Abbreviations}
AVR - axial velocity ratio
CCD = charged coupled device
CC2 - Carleton Cascade Two
CC3 - Carleton Cascade Three
EHM - engine health monitoring
HPFTP= high pressure fuel turbopump
LE - leading edge of roughness band
NBLS = number of blades
PS = blade pressure surface
RMS = root mean square
SS = blade suction surface
SSME - space shuttle main engine
Trans - onset of transition point 


\section{Chapter 1}

\section{INTRODUCTION}

In-service damage to compressor or turbine blades is a common cause of performance deterioration in gas-turbines. Engine Health Monitoring (EHM) systems are routinely used by civilian and military gas-turbine operators to detect gradual or abrupt engine deterioration (eg., Cue and Muir (1991)). Three of the most common forms of blade damage include, blade surface roughening due to fouling, erosion or corrosion; blade tip damage; and leading or trailing edge damage due to foreign-object ingestion or thermal effects. Current EHM systems cannot distinguish between these types of damage. they can only detect the presence of damage, and sometimes even the general area in the engine where the problem exists. Development of an EHM system that can distinguish the different forms of damage is the goal of future EHM systems.

In order to distinguish between the different forms of damage one must understand the effects each form of damage has on the blade aerodynamics. The present study examines the effect of surface roughening on the aerodynamics of turbine blades. Randomly distributed sand grains adhered to the surface of the blades were used to simulate surface roughening. The roughness height, the width of the roughness band, and the location of the band were varied. A combination of experimental measurements and computations were used during the investigation. The experimental part of the present study is largely an extension of earlier measurements made by Abbott (1993). Abbott (1993) compiled a database of profile loss and flow turning information, for randomly distributed roughness applied to the suction surface of cascade turbine blades. The present study expands on this database by examining the effects of both pressure- and 
suction-surface roughness on the losses, flow turning, midspan pressure distribution and the onset of boundary layer transition.

An extensive review of the rough surface flow literature was completed by Abbott (1994). A review of the more recent literature is given in Chapter 2. As well, Chapter 2 contains some brief material on the losses developed in turbines and the cascade terminology used in the present study.

Experimental measurements were conducted on a low-speed, large-scale cascade. In a number of respects, the flow conditions in the test section are idealized compared to the actual machine. That is, all measurements were made under essentially incompressible conditions and with low levels of turbulence in ensity. A description of the experimental apparatus and measurement procedures are presented in Chapter 3.

The experimental cascade was modelled computationally using a viscous/inviscid interaction approach. The objective was to investigate the ability of the computational method to predict the measured results. An outline of the computational method is presented in Chapter 4, along with the computational procedures and practices used in the present study.

The experimental results are presented in the first section of Chapter 5, the computational results are presented and compared to the experimental results in the later portion of Chapter 5. Conclusions and recommendations for future work are presented in Chapter 6. 


\section{Chapter 2}

\section{LITERATURE REVIEW}

The efficiency and operational behaviour of a turbomachine are influenced in great measure by the surface quality of the blading. Blade surface roughening due to fouling, erosion, or corrosion can influence the blade's flow turning ability and losses.

Early research on the effects of surface roughness was conducted using pipe flow and flat plates, only later was research conducted using cascade blades and actual turbines. An extensive review of the rough surface flow literature was completed by Abbott (1993). The following is essentially a summary of that earlier work with emphasis focused on the more recent literature.

\subsection{Cascade Terminology}

Figure 2.1 illustrates the notation commonly used for cascade geometry. The inlet and exit metal angles of the blade are $\beta_{1}$ and $\beta_{2}$. The difference between the inlet design flow angle, $\alpha_{\text {ldes }}$ and the inlet metal angle is often referred to as design incidence, $i_{\text {des }}$. The off-design incidence, $i$, is generally defined as the difference between the inlet flow angle, $\alpha_{1}$, and the inlet desi zn flow angle. The flow deviation angle, $\delta$, is the difference between the outlet flow angle and blade exit metal angle (i.e. $\delta=\alpha_{2}-\beta_{2}$ ). The throat width, $\sigma$, is the perpendicular distance between the trailing edge and the suction surface of the adjacent blade. Other parameters defined in Figure 2.1 include the stagger angle, $\gamma$, blade spacing, $S$, the chord and axial chord, $c$ and $c_{x}$ respectively. 


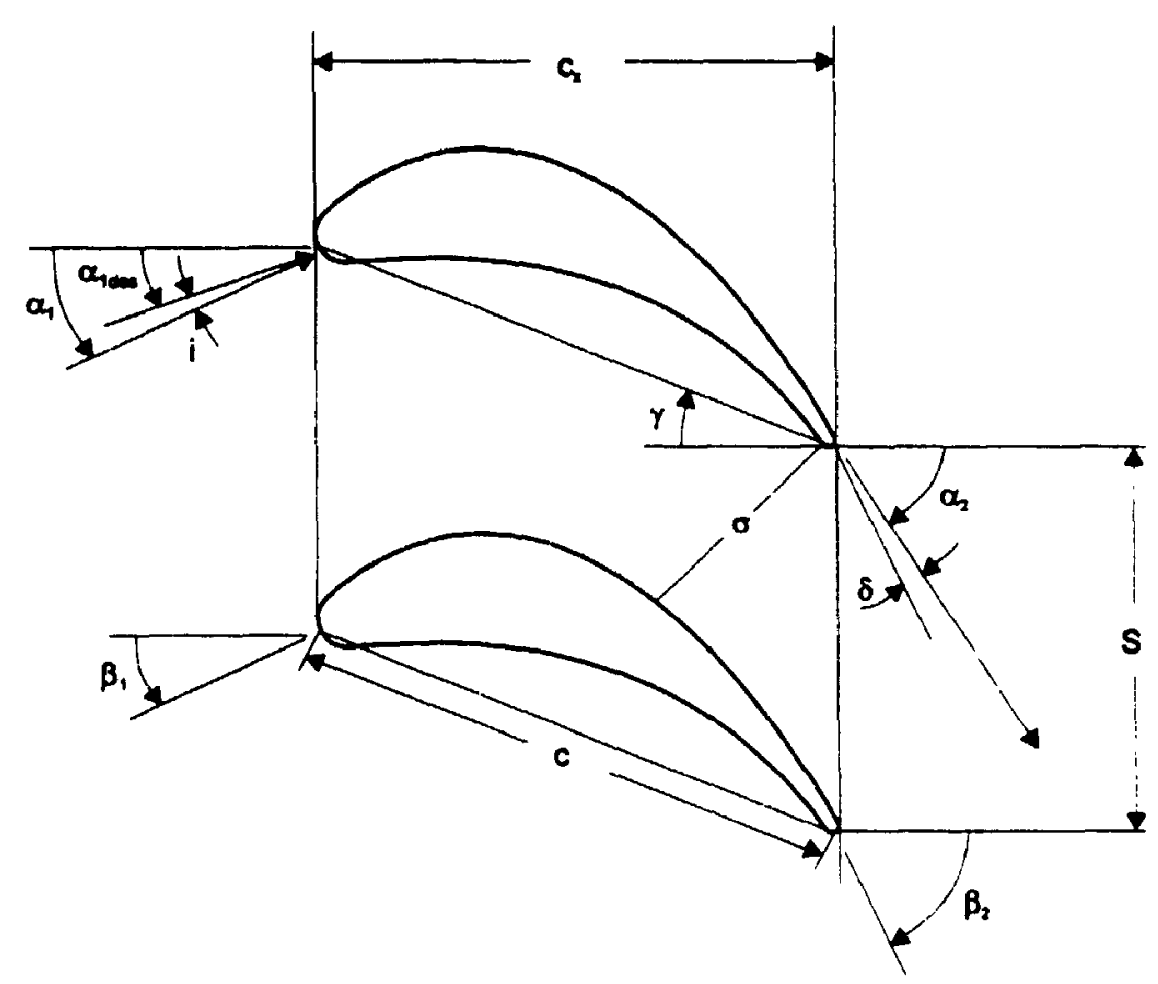

Figure 2.1: Cascade geometry and nomenclature.

\subsection{Losses}

The overall performance of the turbine can only be evaluated on a full scale machine. Assessing the performance of the turbine involves examining the losses developed in the machine. The overall loss of a turbomachine blade row can be divided into the following basic loss components:

1. Profile loss: loss generated in the blade suction and pressure surface 
boundary layers in a uniform two-dimensional flow. The loss due to the finite trailing edge thickness of the blade is usually included.

2. Secondary loss: loss due to the cross flows near the endwall boundaries within the blade row.

3. Tip-leakage loss: loss due to the leakage flow over the blade tips.

This study is mainly concerned with the effect of surface roughness on the profile loss. The two most important results discussed in this study are the total-pressure-loss coefficient, $Y$, and the outlet-flow angle, $\alpha_{2}$, at midspan, in a region of two-dimensional flow. The total-pressure-loss coefficient is defined as the loss in total pressure through the stage normalized with the blade outlet dynamic pressure:

$$
Y=\frac{P_{01}-P_{02}}{P_{02}-P_{2}}
$$

Another parameter which is used to quantify losses is the kinetic-energy-loss coefficient, which is defined as:

$$
\Delta \phi^{2}=1-\frac{V_{2}^{!}}{V_{\text {2isen }}^{2}}
$$

where $V_{\text {2isen }}$ is the isentropic outlet velocity obtained in an isentropic expansion to the same final pressure as the actual process. The conversion from kinetic-energy-loss coefficient to the total-pressure-loss coefficient is given by: 


$$
Y=\frac{\left[1-\frac{\gamma-1}{2} M_{2}^{2}\left(\frac{1}{1-\Delta \phi^{2}}-1\right)\right]^{-\frac{\gamma}{\gamma-1}}-1}{1-\left(1+\frac{\gamma-1}{2} M_{2}^{2}\right)^{-\frac{\gamma}{\gamma-1}}}
$$

which reduces to

$$
Y=\frac{1}{1-\Delta \phi^{2}}-1
$$

for incompressible flows (Tremblay (1989)). In this thesis, the total-pressure-loss coefficient always refers to the profile loss coefficient only.

\subsection{Flow Over Rough Surfaces}

Numerous papers have been written since the early 1900 's examining the effects of surface roughness in both turbomachinery and generai fiuid flow applications. One of the more recent papers pertaining to turbornachinery applications was written by Bammert and Sandstede (1980). They examined the effect of surface roughness on a cascade of turbine blades completely roughened with emery grains. The follcwing measurements were carried out on cascades of varying roughness height: profile midspan static pressure distribution, total losses, outlet-flow angle, and velocity distribution and turbulence in the boundary layer.

Figure 2.2 illustrates the midspan pressure distributions for the profile with and without roughness applied to the surface of the turbine blades. As can be seen, the pressure distribution is not changed very much by the roughness. This confirmed the previous findings of Bammert and Milsch (1972) on compressor blades. On the suction 
side the roughness causes a reduction in velocity, whereas on the pressure surface near the trailing edge, the opposite effect can be observed. The rougher the surface the more pronounced the effects described were.

A small Pitot tube was used to measure the total pressure in the boundary layer at various locations along the profile surface (the tip of the probe was small as compared to the thickness of the boundary layer). Figure 2.3 shows a selection of velocity distributions measured in the boundary layer. The velocity ratio $w / w_{k}$ is plotted as a function of wall distance, $y$; where $w_{k}$ is the undisturbed velocity and $w$ is the local velocity.

- smooth

$\triangle \quad K / C=0.00057$

$\nabla K / C=0.00102$

$\checkmark \quad k / c=0.00331$

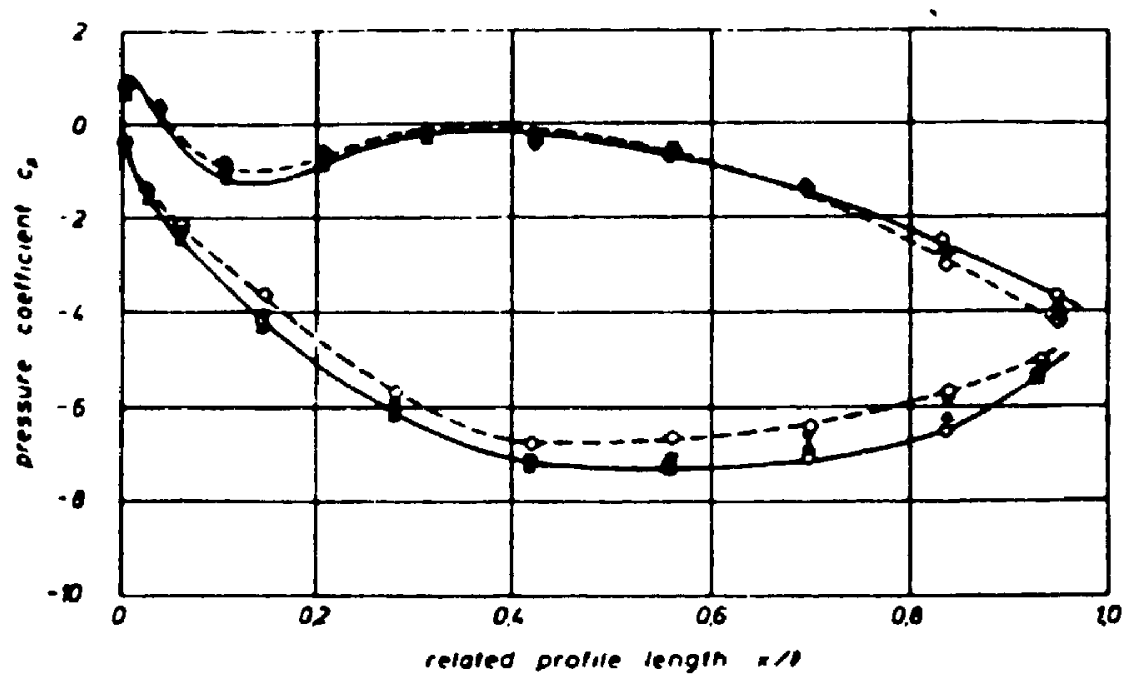

Figure 2.2: Static pressure distribution for various roughness t.eights - reproduced from Bammert and Sandstede (1980). 


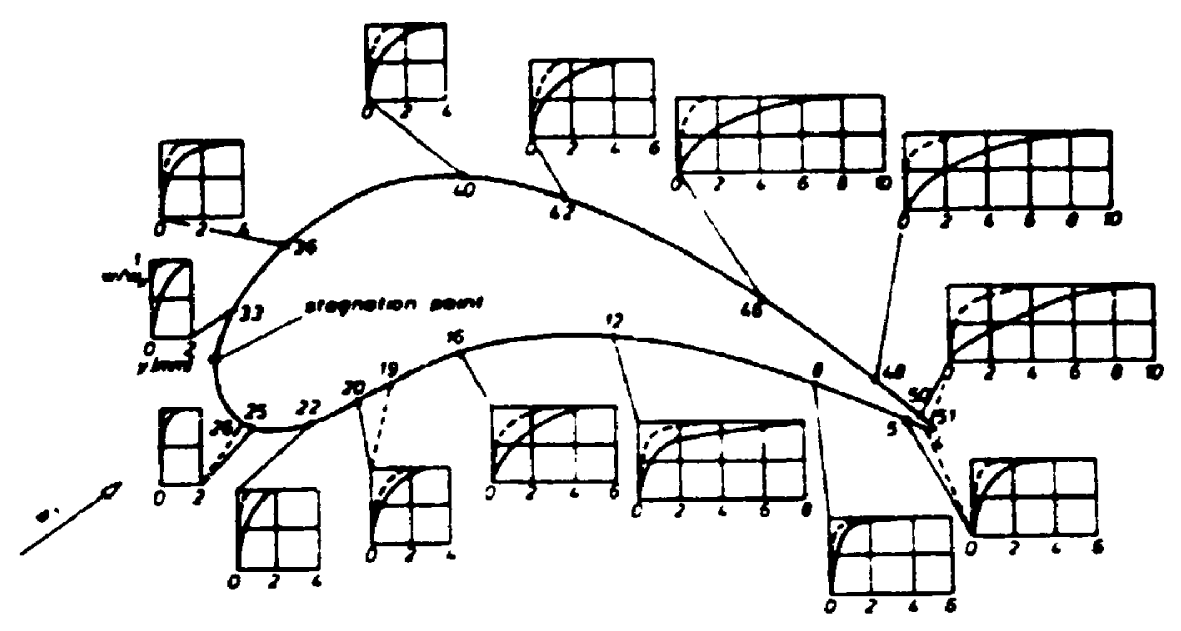

Figure 2.3: Velocity distribution along profile: ( - ) smooth, (-----) rough (k/c = 0.00331 ) - reproduced from Bammert and Sandstede (1980).
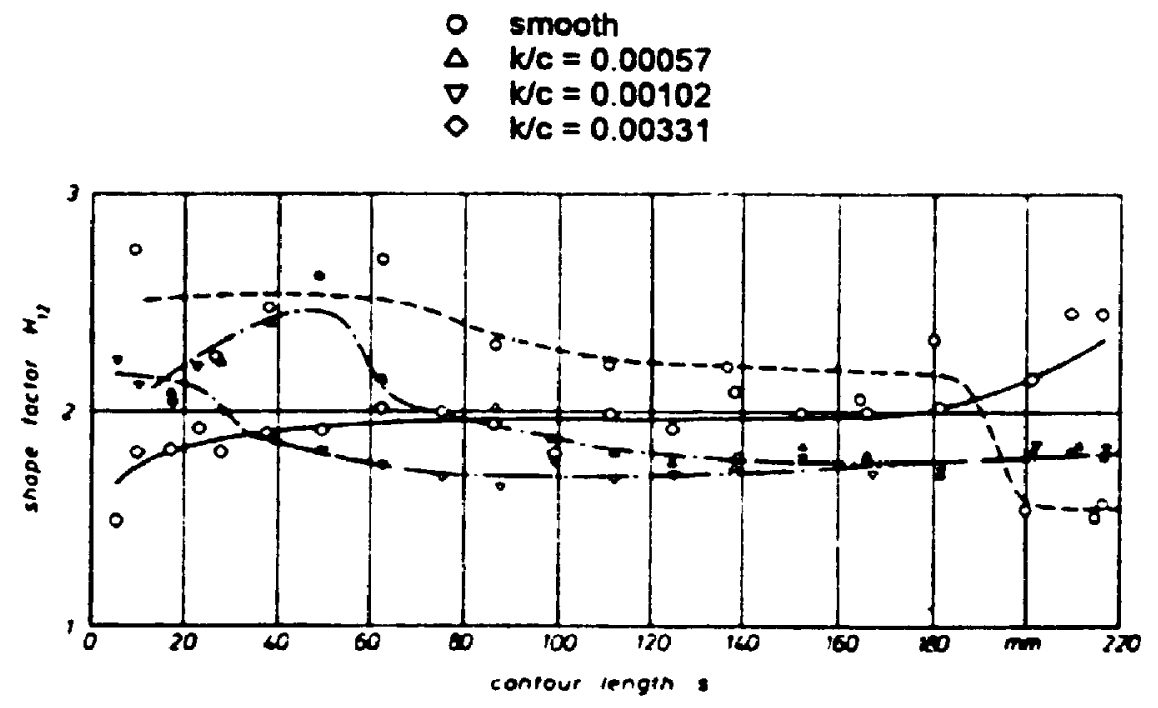

Figure 2.4: Shape factor on the suction surface - reproduced from Bammert and Sandstede (1980). 

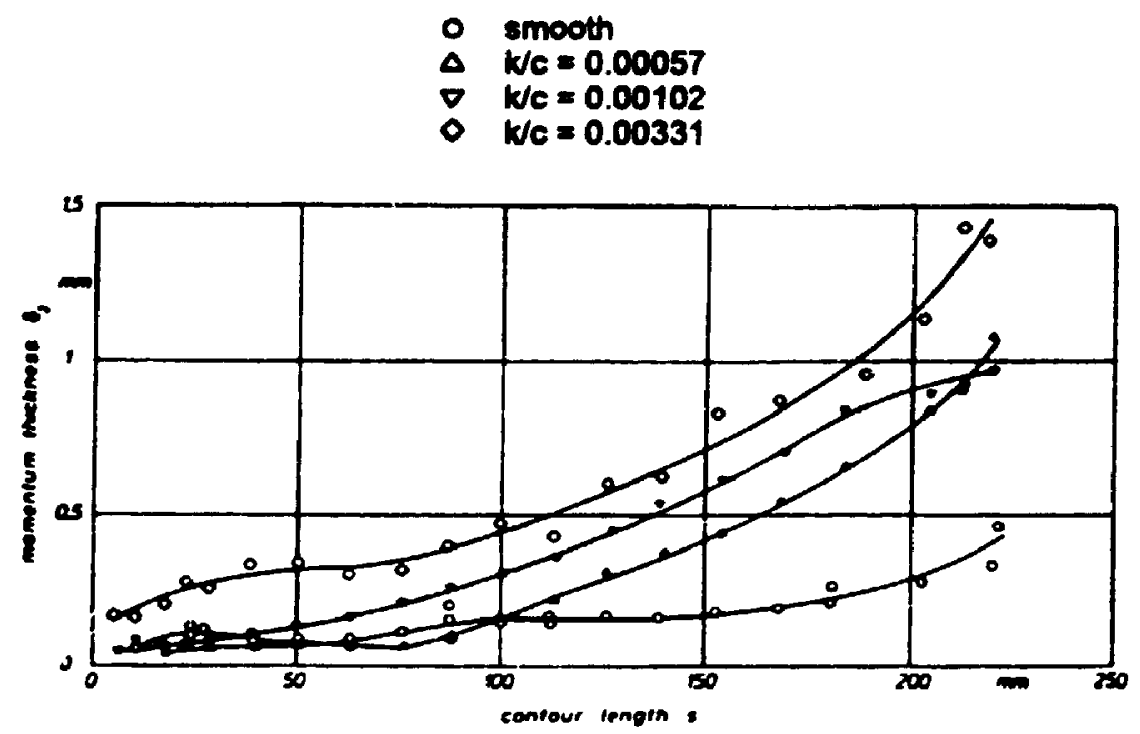

Figure 2.5: Momentum thickness on the suction surface - reproduced from Bammert and Sandstede (1980).

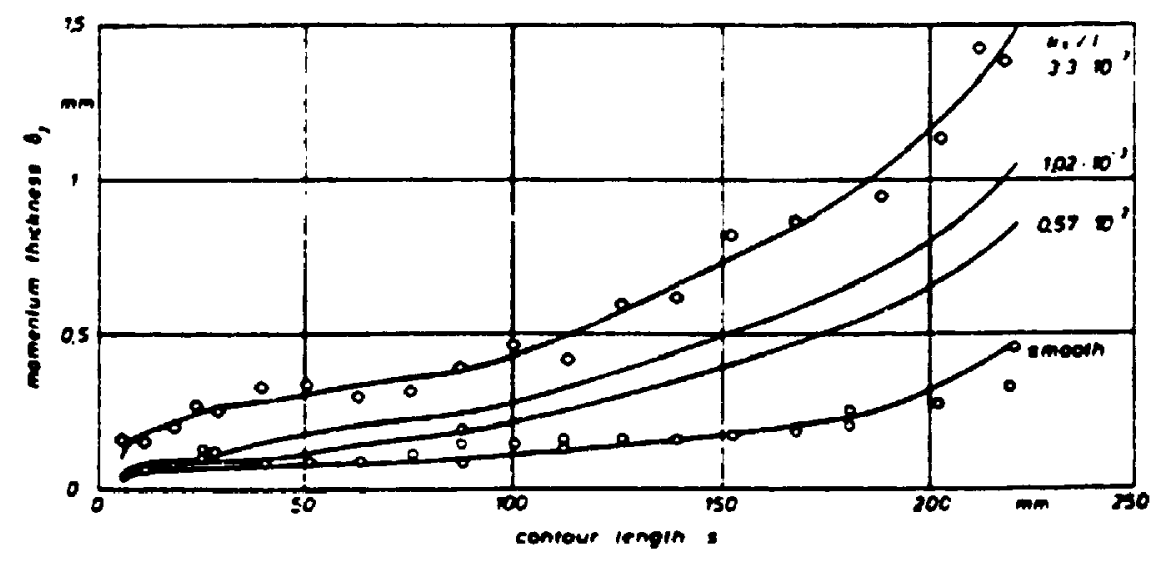

Figure 2.6: Calculated momentum thickness on the suction surface. Measured points are represented by the open symbols - reproduced from Bammert and Sandstede (1980). 
The shape factor and momentum thickness are calculated from these data and are plotted for the suction surface in Figures 2.4 and 2.5, respectively. The transition point was determined from the shape factor, that is when $H$ fell below 2 transition was assumed to have occurred. As the roughness size was increased the transition point moved further upstream.

Theoretical calculations of the boundary layer were performed for the measured pressure distributions. The experimentally determined shape factor was used to determine the transition point for each of the roughness grades. An integral methor was used based on the skin friction crefficient, $c_{p}$ on a flat plate. The skin friction coefficients were calculated for laminar flow according to

$$
c_{f}=\frac{1.328}{\sqrt{\operatorname{Re}}} k_{r}
$$

and for a turbulent flow with a smooth surface according to

$$
c_{f}=0.455(\log (R e))^{-2.58}
$$

and for a rough surface according to

$$
c_{f}=0.418\left(2+\log \left(\frac{c}{k}\right)\right)^{-2.53} k_{r}
$$

where $R e$ is the Reynolds number at inlet referred to the blade chord $c, k$ is the thickness of the sand roughness, $k_{r}$ is a roughness correction factor. The calculated results agreed satisfactorily with the measurements only for a smooth surface. The correction factor, $k_{r}$, was used for the rough surfaces, due to the calculated results having underpredicted the affects of roughners, and is defined as 


$$
k_{r}=5.7+0.48 \ln \left(\frac{k}{c}\right)
$$

for the laminar boundary layer and

$$
k_{r}=3.4+0.26 \ln \left(\frac{k}{c}\right)
$$

for turbulent boundary layers.

The solid lines shown Figure 2.6 represent the computational predictions of momentum thickness. The overall losses and outlet flow angle were determined from downstream measurements in the wake. The overall losses of the cascade rose from $1.85 \%$ for a smooth profile to $6.39 \%$ for the largest roughness $(k / c=0.00331)$. The outlet flow angle results were not presented or discussed.

Bynton et al. (1992) performed a 'case study' of roughness effects on the Space Shuttle Main Engine (SSME) High Pressure Fuel Turbopump (HPFTP). A full scale m.udel of the two-stage SSME HPFTP reaction turbine in a cold test rig at the Marshall Space Flight Centre was tested with smooth and rough surface blades. The smooth blade results were obtained by polishing rotor blades which had a rough NiCrAlY coating. The rough coated blades had a surface finish of $10.16 \mu \mathrm{m}$ and the smooth blades a surface finish of $0.75 \mu \mathrm{m}$. Turbine efficiency and flow characteristics were compared at design and off-design points.

The efficiency improvement of the smooth blades compared to the rough blades is shown in Figure 2.7. The efficiency increase at the 104\% RPL (Rated Power Level) equivalent condition was $2.5 \%$ for the smooth blades. Table 2.1 summarizes the rough and smooth blade averages for various parameters measured at 104\% RPL. 


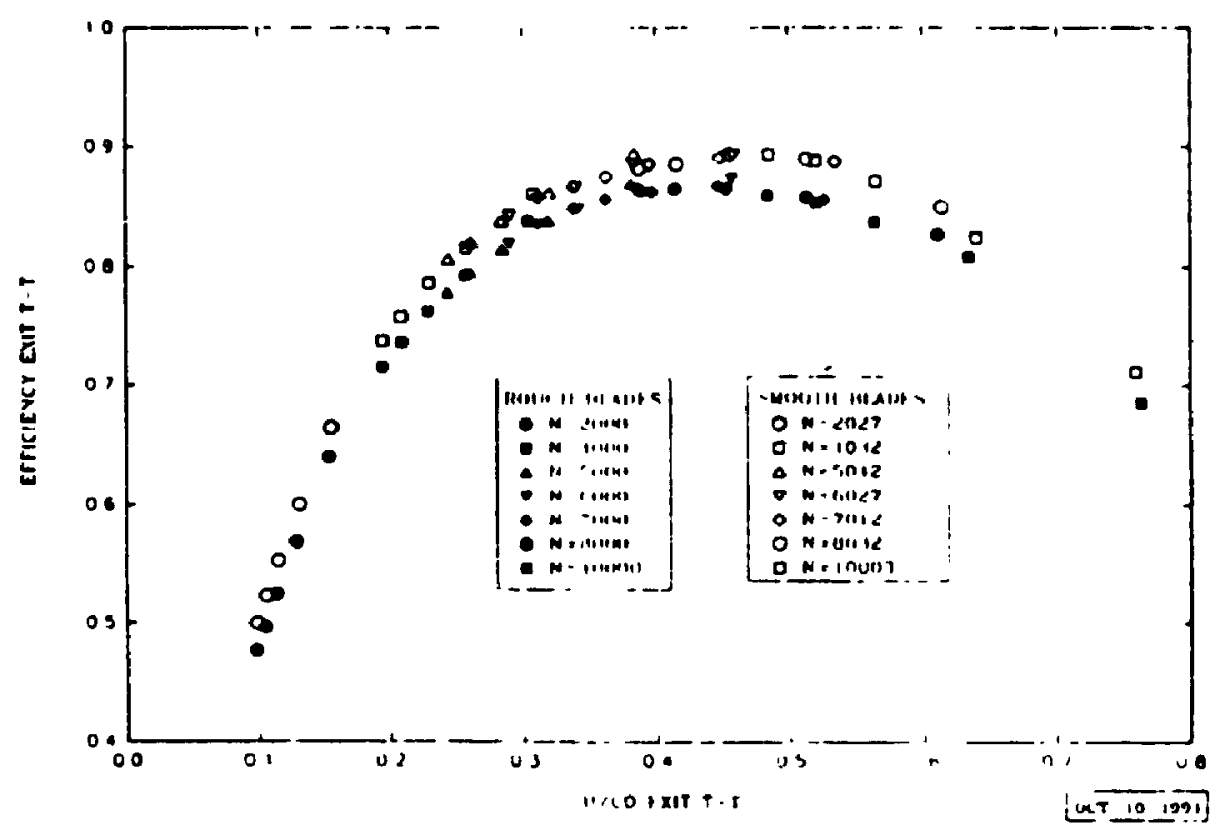

Figure 2.7: Efficiency for smooth and rough blades (where: U-mean blade speed, Co-overall isentropic velocity total-total) - reproduced from Boynton et al. (1992).

\begin{tabular}{|c|c|c|c|c|}
\hline \multicolumn{2}{|l|}{ Rovor Blades } & \multirow{2}{*}{$\begin{array}{l}\text { Roungh } \\
\text { Blodes } \\
\text { Averaexs } \\
\text { math } \\
\text { Mits }\end{array}$} & \multirow{2}{*}{$\begin{array}{l}\text { Smooth } \\
\text { aledes } \\
\text { trerages } \\
1041 \\
\operatorname{mis} 81\end{array}$} & \multirow[t]{2}{*}{ Derita } \\
\hline PTV & $\begin{array}{c}\text { PSIAT } \\
\text { KPaI }\end{array}$ & & & \\
\hline$\pi \pi$ & $\begin{array}{c}R \\
|h|\end{array}$ & $\begin{array}{l}506 \\
\text { in } 21\end{array}$ & 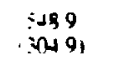 & \\
\hline Speed &. $\mathrm{om}$ & $-m^{-}$ & $\cdot m 8$ & \\
\hline PRT-T ETIt & - & $H i$ &.+67 & \\
\hline $\mathrm{LC}_{13}$ & - & $11: 2$ & $113=$ & \\
\hline Elt-Deira trmo & - & $(18507)$ & .18792 & $-\geq+3 \mathrm{c}$ \\
\hline Elf-Inta & - & 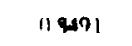 & $1372=$ & $-2=$ \\
\hline Eft-tora mest & - & 08311 & 08550 & $-28 . e_{c}$ \\
\hline 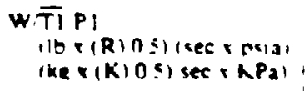 & & $\begin{array}{l}3290 \\
1016\end{array}$ & $\begin{array}{l}749 \\
1691\end{array}$ & $\begin{array}{l}-464 \% \\
-464 \%\end{array}$ \\
\hline Ourlet swril ill & & $-2 i$ & +17 & $+38 \mathrm{deg}$ \\
\hline \multirow{2}{*}{\multicolumn{5}{|c|}{$\begin{array}{l}\text { (1) Outlet swirl in degrees trom axial. dverage of cobras } 3 \text { and } 4 \text { at } 525 \% \\
\text { span. positive in direction of rotor rotation } \\
\text { Where }\end{array}$}} \\
\hline & & & & \\
\hline \multicolumn{5}{|c|}{ 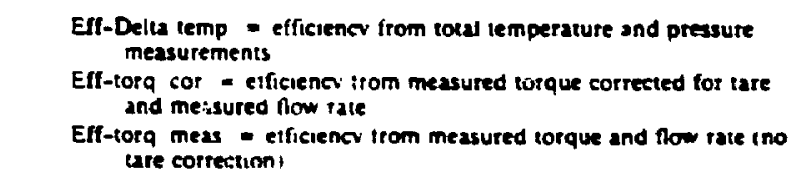 } \\
\hline
\end{tabular}

Table 2.1: Smooth and rough blade averages at $104 \%$ RPL equivalent conditions reproduced from Boynton et al. (1992). 
Boyle (1993) used a Nevier-Stokes analysis to predict the change in turbine efficiency resulting from changes in blade surface roughness or incidence angles. The Navier-Stokes analysis used the mixing length turbulence model of Cebeci and Chang (1978) to determine the effects of surface roughness. The results of the midspan Navier-Stokes analysis were combined with those from a quasi-3D flow analysis code to predict turbine performance. Verification of this approach was done by comparing the results with the experimental data of Boynton et al. (1992).

Figure 2.8 shows a comparison of the turbine efficiency using a curve fit of the Navier-Stokes results. The predicted effect of surface roughness on turbine efficiency was strongly affected by the choice of parameters in the rough surface turbulent eddy viscosity model. Conventional values used in the model resulted in poor predictions of the surface roughness effect on turbine efficiency. Manipulations of the parameters was necessary to get good agreement with the experimental data.

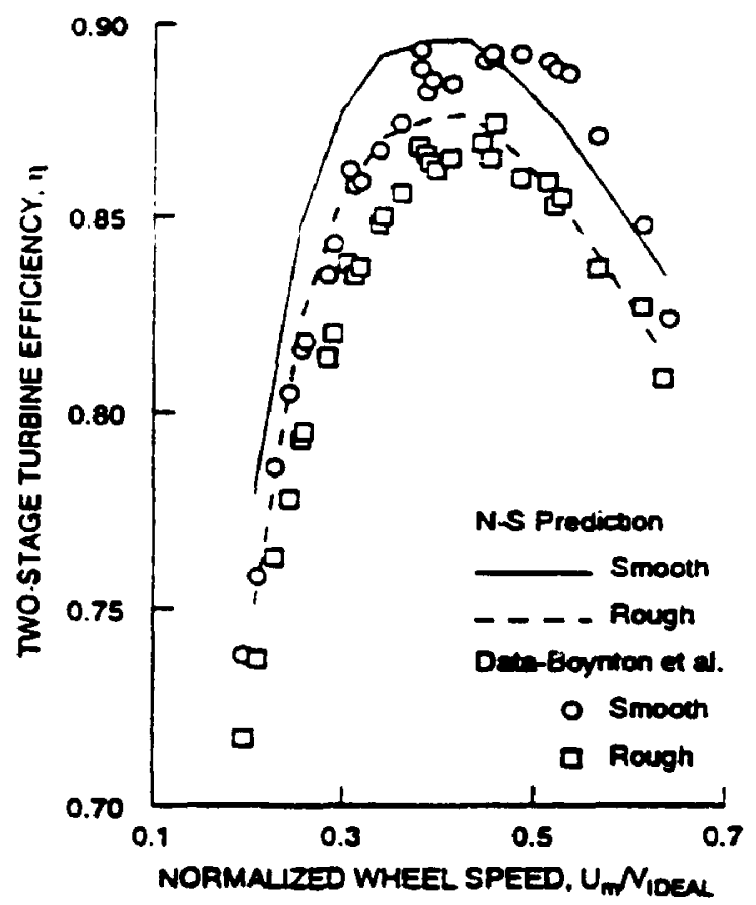

Figure 2.8: Smooth and rough blade predicted and measured efficiency (where: $\mathrm{U}_{\mathrm{m}}-$ mean wheel speed, $\mathrm{V}_{\mathrm{DEAL}}-$ ideal isentropic velocity) - reproduced from Boyle (1993). 
Suder et al. (1994) investigated the effects of adding thickness and roughness to a high speed axial compressor rotor. A thick rough coating with a surface finish of 2.54 3.18 RMS $\mu m$ was applied to the pressure and suction surface of the rotor blades. To separate the effects of thickness and roughness, a smooth coating of equal thickness was also applied to the blades. The smooth coating surface finish was $0.254-0.508$ RMS $\mu \mathrm{m}$ compared to the bare metal blade surface finish of $0.508 \mathrm{RMS} \mu \mathrm{m}$. Both coatings were then applied to different portions of the blade surface to determine which portion of the airfoil was most sensitive to thickness/roughness variations.

Aerodynamic performance measurements were performed on the low aspect ratio transonic core, test compressor inlet stage. A number of smooth and rough coating configurations were investigated over a wide range of operating conditions. To complement the experimental measurements a quasi-3D Navier-Stokes flow solver was used to provide insight into the flow development.

The smooth- and rough-coate: performance was compared to the baseline performance in Figure 2.9. Also sh wn in Figure 2.9, is an approximate throttle line along which aircraft engine fans and compressors operate. As the compressor deteriorates in service, the operating point will move along a constant-throttle line. The efficiency loss along the approximate throttle line shown is 3.5 points for the smooth coating and 6 points for the rough coating.

Figure 2.10 shows the decrease in design speed pressure ratio for partially coated blades. The full coverage of rough paint, case I. gave the largest deterioration in performance. There was little deterioration in performance when the leading edge was left uncoated, as shown by Cases D, E. F. Examining the results for Cases I in which the blades were fully covered and Case $\mathrm{H}$ in which the leading edge and $50 \%$ of the suction surfaces were covered revealed that pressure-surface roughness had little effect on performance. Cases $\mathrm{H}$ and $\mathrm{G}$ differed only in the extent of the roughness on the suction surface, (i.e. an extra $40 \%$ of the suction surface was covered in Case $\mathrm{H}$ ) and resulted in 
a larger impact on performance degradation. Case $\mathrm{C}$, not shown in Figure 2.10, was geometrically identical to Case $\mathrm{G}$ although Case $\mathrm{C}$ was for a smooth coating and Case G for a rough coating. A significantly larger performance loss resulted for the rough coating in Case G.

To complement the experimental measurements, a quasi-3D Navier-Stokes flow solver was used. The results were generated using a thin-layer Navier-Stokes analysis code which employed the Baldwin-Lomax algebraic turbulence model (1978). Surface roughness was modelled by increasing the turbulent mixing length using the approach of Cebeci and Chang (1978). Transition was modelled by calculating a turbulent viscosity profile along the blade.

Figure 2.11 shows the predicted results for the smooth-coated blade and the baseline blade compared to experimental data. For a smooth leading edge the predicted performance deterioration between the baseline blade $\left({ }^{*}\right)$ and the smooth-coated blade $(\Delta)$ is far less than that observed experimentally. Predicted transition on the smooth coated blades occurred naturally at $36 \%$ chord on the suction surface and $29 \%$ chord on the pressure surface. By forcing transition to occur on both surfaces at the leading edge resulted in closer agreement with the measured results. This result indicated that the performance deterioration observed for the smooth-coated blade was not due to an increase in blade thickness but rather from the boundary layer being tripped at the leading edge. The smooth coating was relatively soft and therefore resulted in a smooth-coated blade which was more susceptible to damage at the leading edge than was the baseline blade.

The predicted results for the rough-coated and baseline blade are compared to the experimental data in Figure 2.12. The open symbol curve represents a roughness equal to that measured in the experiment, while the solid symbol curve represents a roughness height which is four times that used in the experiment. As can be seen, the compressor performance characteristics are sensitive to the addition of surface roughness although the model significantly underpredicts the performance changes measured in the experiment. 


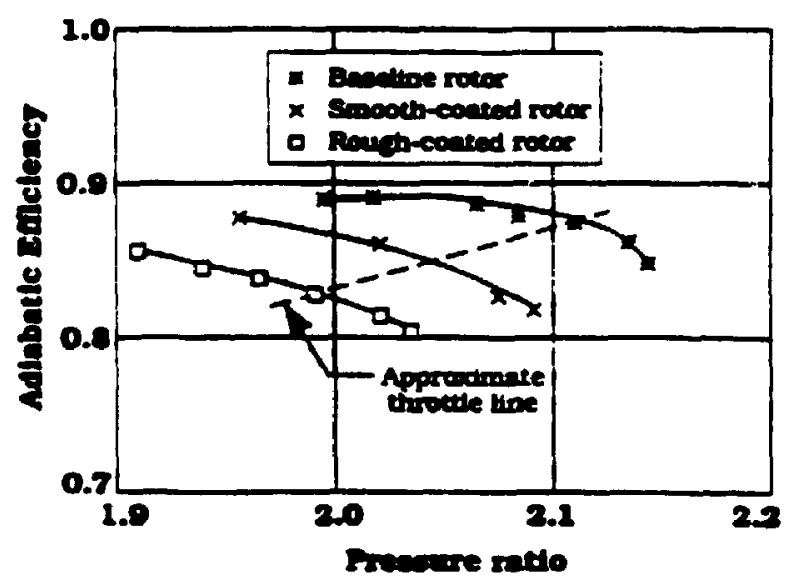

Figure 2.9: Efficiency-pressure ratio characteristics at design speed - reproduced from Suder et al. (1994).

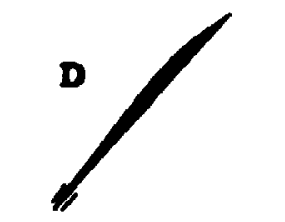

$\Delta$

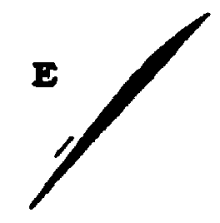

varear ss

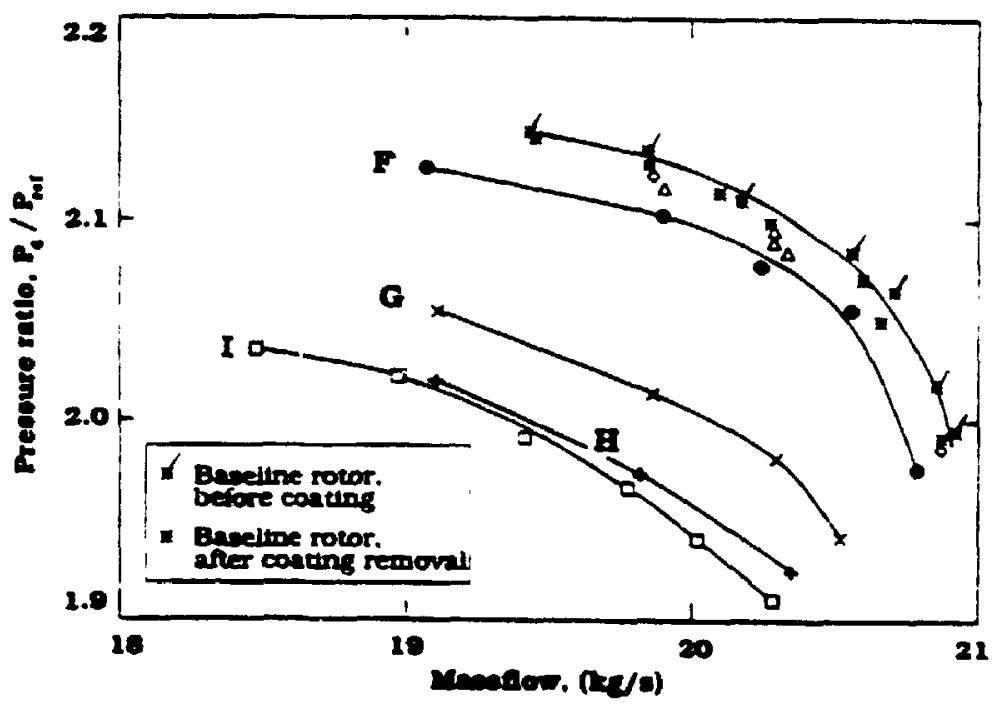

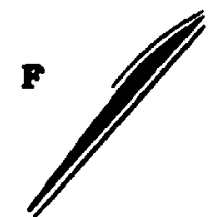

so-100m en: 2100m to

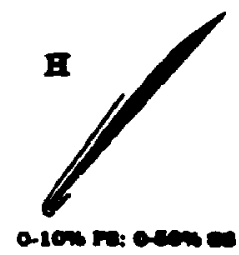

+ orsen Hi cenos

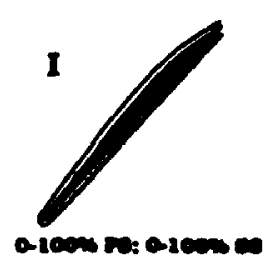

Figure 2.10: Pressure rise characteristics for the rough coatings at design speed reproduced from Suder et al. (1994). 


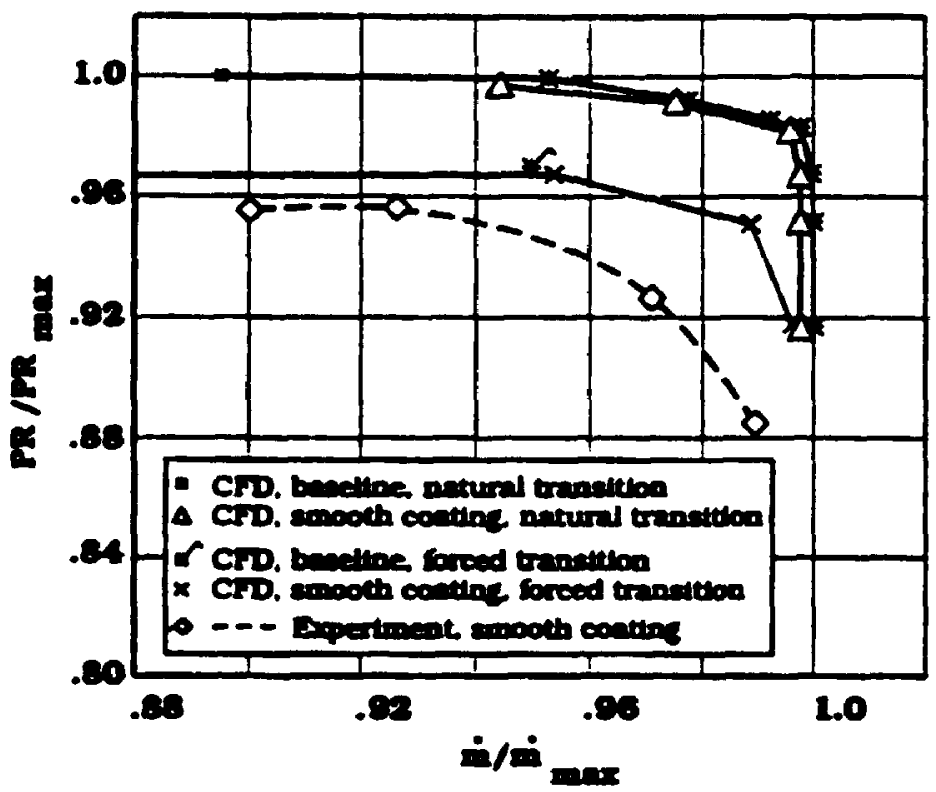

Figure 2.11: Comparison between predicted and measured pressure rise versus massflow characteristics at $70 \%$ span for the smooth coated blade reproduced from Suder et al. (1994).

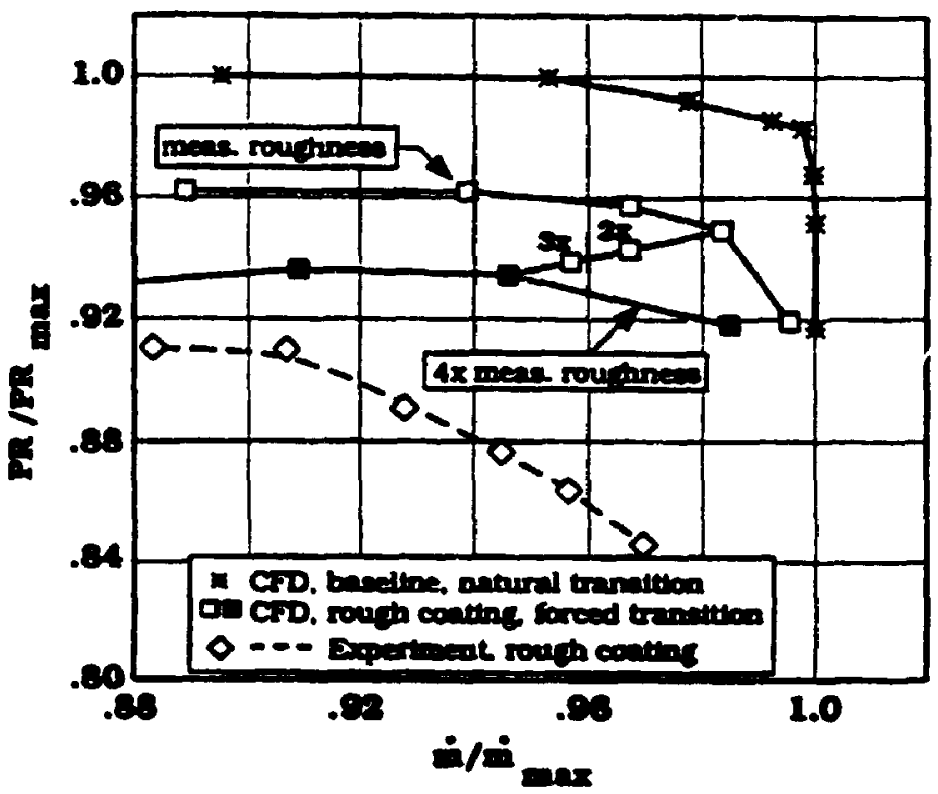

Figure 2.12: Comparison between predicted and measured pressure rise versus massflow characteristics at $70 \%$ span for the rough coated blade reproduced from Suder et al. (1994). 


\section{Chapter 3}

\section{DESCRIPTION OF APPARATUS AND PROCEDURE}

\subsection{Wind Tunnel}

The wind tunnel used in this research is shown in Figure 3.1; it was commissioned by Rodger (1992). As shown, the wind tunnel is comprised of a centrifugal fan, followed by a diffuser, a large settling chamber and a contraction. The centrifugal fan is driven by an AC motor. The motor speed is controlled by a remote variable frequency controller. The fan forces air through the diffuser comprised of five screens. Following the diffuser, the air is passed through a large settling chamber containing four screens. The four screens are used primarily to remove non-uniformities in the flow. From the settling chamber, air enters a 14:1 contraction. The maximum exit velocity from the contraction is about $30 \mathrm{~m} / \mathrm{s}$ so that conditions in the test section are essentially incompressible. The free stream turbulent intensity at the test section inlet is about $0.3 \%$. Detailed information on the wind tunnel components can be found in the work of Rodger (1992).

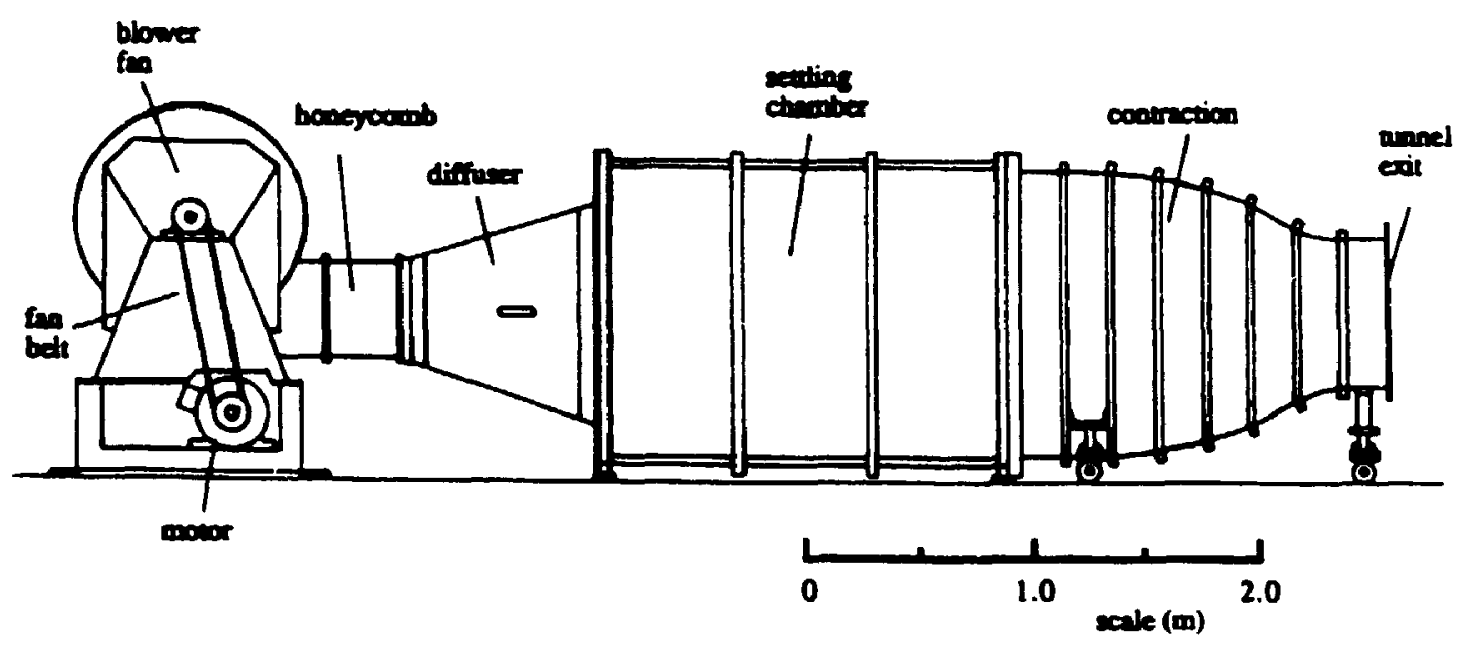

Figure 3.1: Schematic of the wind tunnel - reproduced from Rodger (1992). 


\subsubsection{Cascade Test Section}

The cascade test section used for the present study was designed by Gonbie (1989) and is shown in Figure 3.2. The test section inlet flange is rectangular and is bolted to the outlet flange of the wind tunnel contraction. The entire assembly is mounted on wheels to allow it to be removed from the tunnel outlet. Two vertical boundary layer bleed slots, near the inlet of the test section, help reduce the thickness of boundary layers developed inside the contraction.

The linear cascade of five blades is mounted on a tumtable to allow for measurements at design and off-design angles. The turntable can be rotated in excess of $\pm 45^{\circ}$. A protractor and vernier scale engraved on the back of the turntable allows a positioning accuracy to $0.1^{\circ}$. The blades are bolted at a fixed stagger angle to a removable mounting plate that is in tum bolted to the tumtable. The test section is equipped with side flaps, tailboards, and endplates. Flow uniformity at the inlet to the cascade, and periodicity at the outlet are achieved by adjusting the side flaps and tailboards. The two sliding endplates can be adjusted such that the passage between them is slightly divergent; they are adjusted such that the mean axial velocity at midspan at inlet and exit of the cascade are equal, as required for two-dimensional flow in the midspan region. These endplates are described in more detail by Rodger (1992).

\subsubsection{Cascade Blades}

The cascade of blades, Carleton Cascade Three (CC3), used in the present research, was scaled from a Pratt and Whitney Canada power turbine rotor at mid height. The cascade has been used extensively to study the off-design losses and blade damage in turbines (eg., Whitehouse (1993), Issacs (1994), Abbott (1993)). Figure 3.3 shows the geometric parameters of the cascade of five blades and the inlet and outlet measuring 


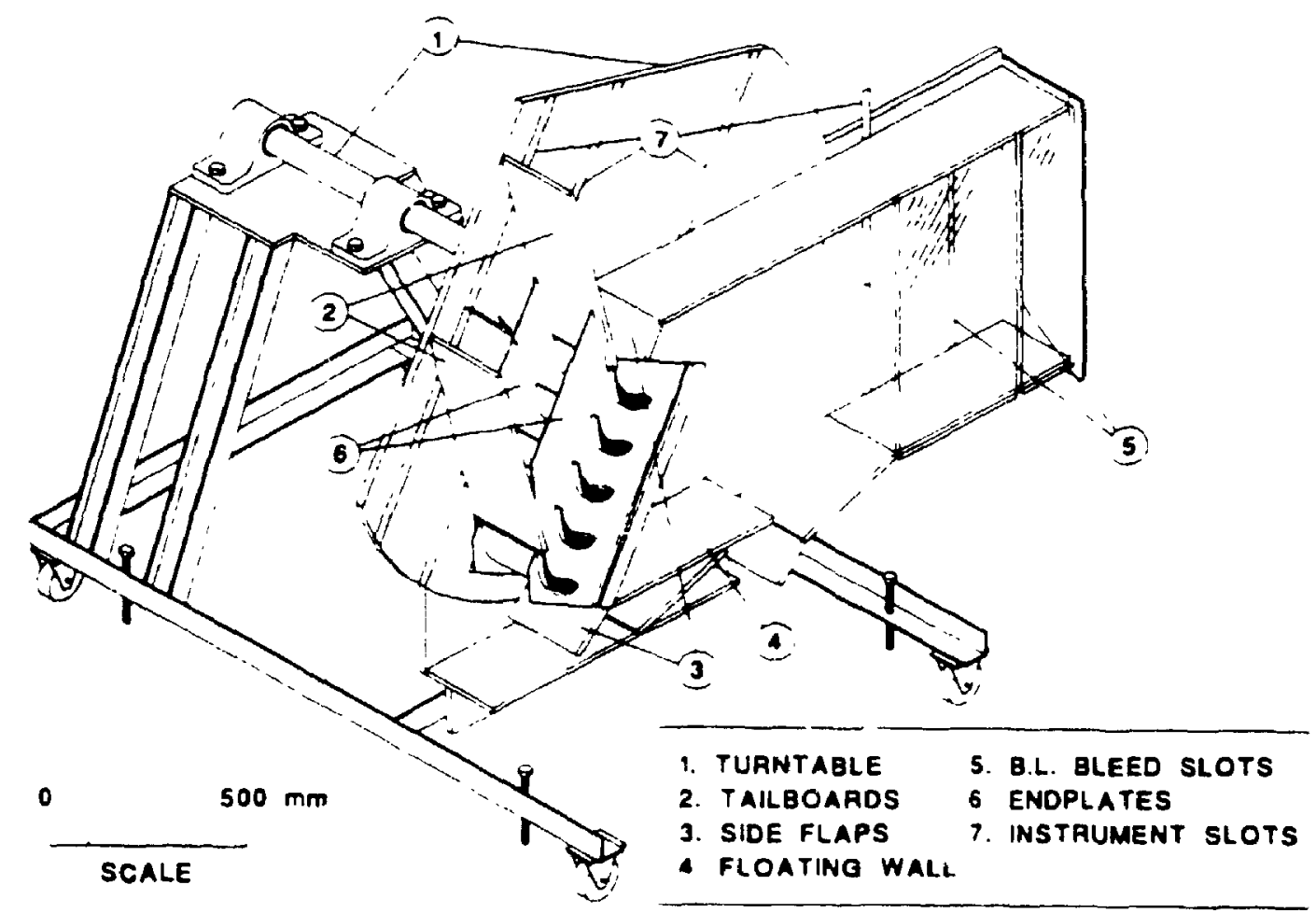

Figure 3.2: Variable incidence cascade test section - reproduced from Sjolander et al. (1993). 


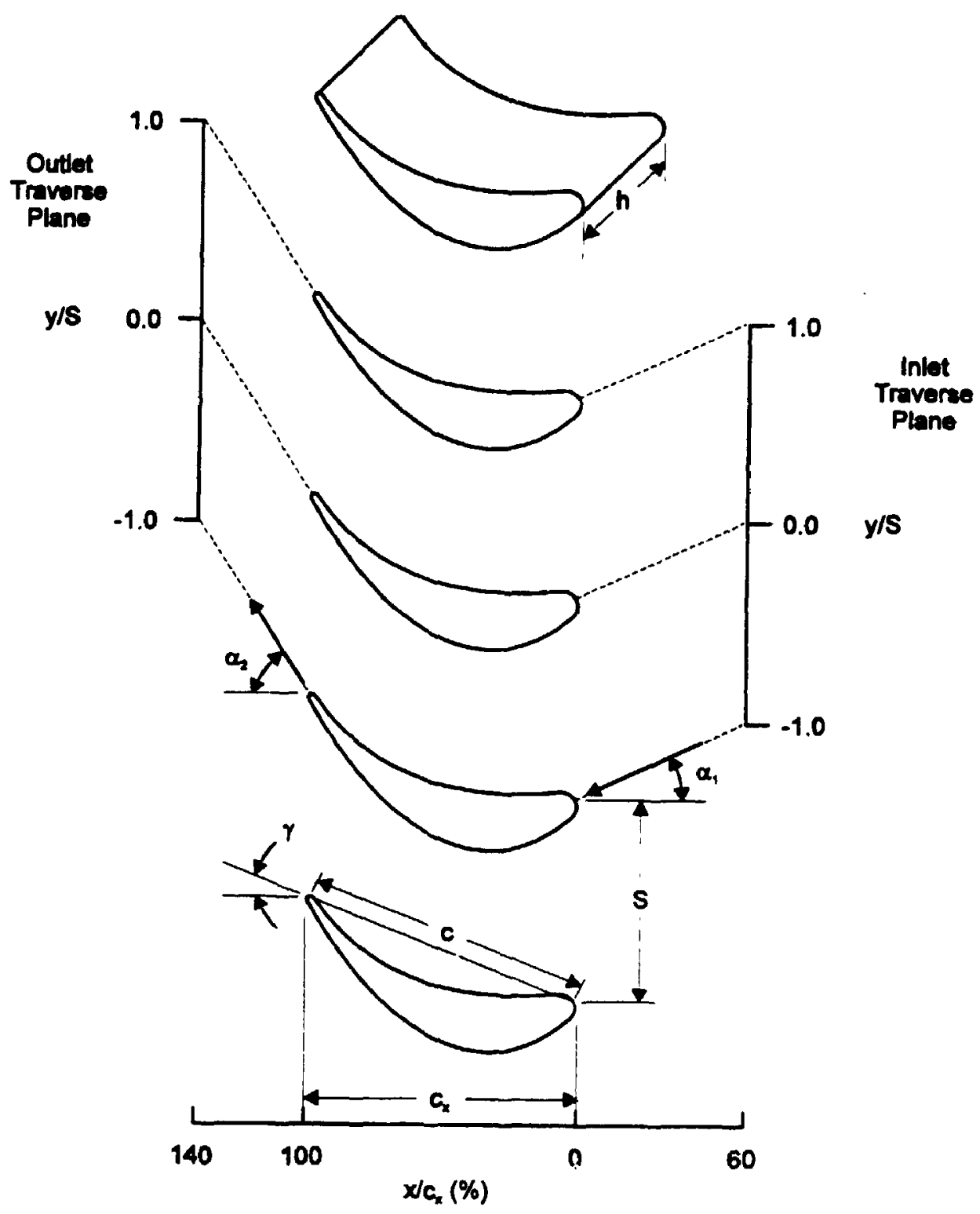

Chord Length, c Axial Chord Length, $c_{\text {. }}$ $162.3 \mathrm{~mm}$ Blade Span, $h$ Blade Specing, s Leading Edgo Diameter Trailing Edgo Diemoter Stageer Angle, $\gamma$ Inlet Flow Angle $149.4 \mathrm{~mm}$ : $200 \mathrm{~mm}$ : $111 \mathrm{~mm}$ : $16.7 \mathrm{~mm}$

: $4.2 \mathrm{~mm}$

Outiot Flow Angle

: $21.6 \mathrm{deg}$.

: $\boldsymbol{\alpha}_{\mathbf{1}}$

$\alpha_{2}$

Figure 3.3: Cascade geometry and measuring plane nomenclature. 
planes. The coordinates used to describe the profile are contained in Appendix A. Details of the design and manufacturing process can be found in Whitehouse (1993).

A sixth identical blade was used to measure the stacic pressure distribution on the blade surface. The outer half of the blade contains 10 spanwise rows of 43 static pressure taps per row. Each spanwise row of taps shares the same internal channel, therefore only one row of taps can be exposed at any one time. The midspan pressure distribution of the blade was of concern in the present study therefore the remaining nine rows of taps were covered with tape. The static pressure tap coordinates and orientation along with graphical depictions are contained in Appendix B. Pressure tap pairs 23 and 27, 24 and 26, 42 and 43 share the same channel inside the blade due to manufacturing difficulties. Therefore, one tap of each pair must be taped over during sampling. After the open holes have been sampled the wind tunnel is stopped, the tape is removed, and the previously exposed taps are covered. The wind tunnel is then restarted and the sampling is resumed.

\subsection{Data Acquisition Equipment and Procedures}

\subsubsection{Data Acquisition Equipment}

The arrangement of the data acquisition equipment is shown in Fig'je 3.4. A three-hole probe is used to measure the flow parameters of velocity, a. gle and total pressure. The three-hole probe will be detailed in Section 3.2.2.

As shown in Figure 3.4, each tube of the three-hole probe is connected to the inputs of a scani-valve system. The scani-valve is a multi-port pneumatic switching device with 48 inputs and one output. The measured pressures from the output of the 


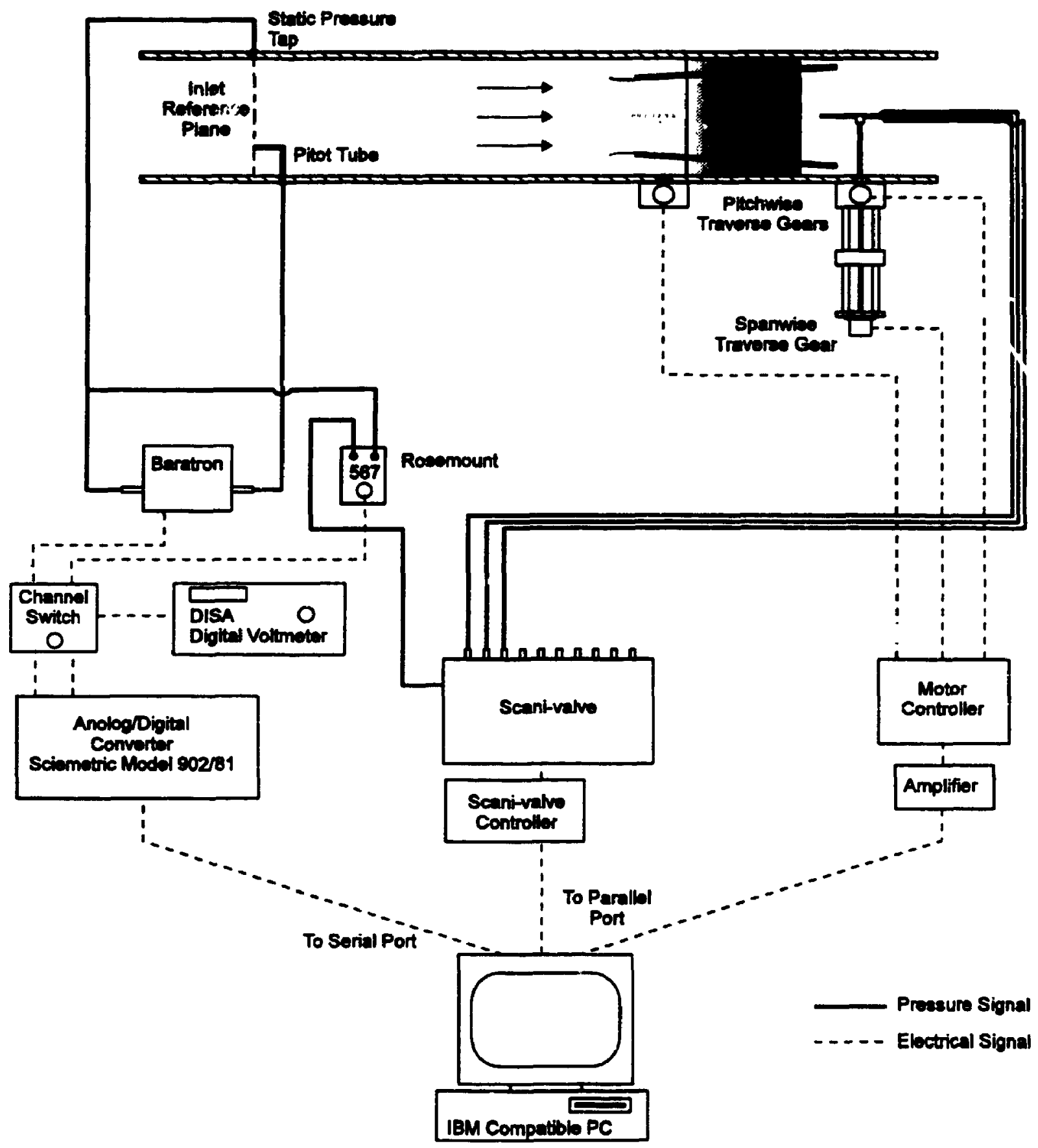

Figure 3.4: Data acquisition system set up for three-hole pressure probe measurements. 
scani-valve are converted to analog voltage signals using a Rosemount (Model 831 A3 serial\# 567) differential pressure transducer with a range of $\pm 3.43 \mathrm{KPa}$ ( $\pm 0.5 \mathrm{psid})$ and a full scale output of \pm 2.5 volts DC. A Baratron (Model 220DC. serial\# 79508-2) differential pressure transducer with a range of $\pm 6.86 \mathrm{KPa}( \pm 1.0 \mathrm{psid})$ and a full scale output of \pm 10.0 volts $D C$ is used to measure the reference dynamic pressure. The two transducers were calibrated prior to this research and checked periodically. The calibration was performed using a micromanometer, a voltmeter and two $100 \mathrm{CC}$ syringes in parallel as a pressure source. The resultant calibration curves are presented in Appendix C.

The voltage signals from the transducers are then sent to a Sciemetric Instruments 12-bit Analog/Digital converter (Model 81/902, serial\# 9023J/19006). The transducer voltages can be simultaneously monitored using a DISA 55D31 time-averaging voltmeter. A manual switching device allows the user to monitor either the reference dynamic pressure or the output pressure of the scani-valve. The 12-bit Analog/Digital converter is connected directly to the microcomputer via the serial port and communication is carried out with a special set of commands. The pressure signal received is then adjusted for the zero reading and transformed into a pressure value using the calibration coefficient of the transducer. The pressure value is then normalized by the reference dynamic pressure at the test section inlet. The sampling rate is roughly 5 samples per second.

Flow measurements are made in planes upstream and downstream of the cascade using three-hole pressure probes. The probe locations are adjusted using stepper-motor driven traverse gears. The traverse gears are mounted on the outside wall of the turntable. Removable probe mounting rods are attached to the traverse geal: ind pass through the instrument slots machined in the turntable wall. Two traverse gears can be mounted in the downstream measurement planes at one time. One traverse gear allows for pitchwise measurements the other for spanwise measurements. The stepping motors are powered by a motor controller unit connected to the microcomputer parallel port via an amplifier. 
The data acquisition system is controlled by the microcomputer using the Pascal program THP2-35. This program is described by Whitehouse (1993).

\subsubsection{Three-Hole Pressure Probes}

Flow parameters were measured at the inlet and outlet of the cascade using threehole pressure probes aligned with the nominal flow direction. Figure 3.5, shows a drawing of the three-hole probes used in the present research. The shorter of the two probes was used to make measurements at the inlet plane which is $60 \%$ of axial chord upstream of the leading edge. Outlet measurements were made with the larger probe in a plane $140 \%$ of axial chord downstream of the leading edge. The shorter probe was necessary at the inlet plane to allow for measurements close to the cascade without the probe tail interfering with the leading edges of the blades. A third probe was designed and built in the early stages of the experimental work. The probe was designed with smaller tubing than the two probes shown in Figure 3.5. Appendix F contains detailed drawings of all three probes.

\subsubsection{Calibration and Sampling Procedure}

A schematic diagram of the calibration apparatus and data acquisition system is shown in Figure 3.6. Calibration of the three-hole pressure probe was performed by the author in May 1994, and September 1994. The probes had been calibrated previously by Abbott (1993) in May 1993. The results indicated that the calibrations remained essentially unchanged over the aforementioned periods.

The probe calibration was carried out in the inviscid core of a jet flow, according to the method established by Lewis (1966). The core velocity was approximately equal 

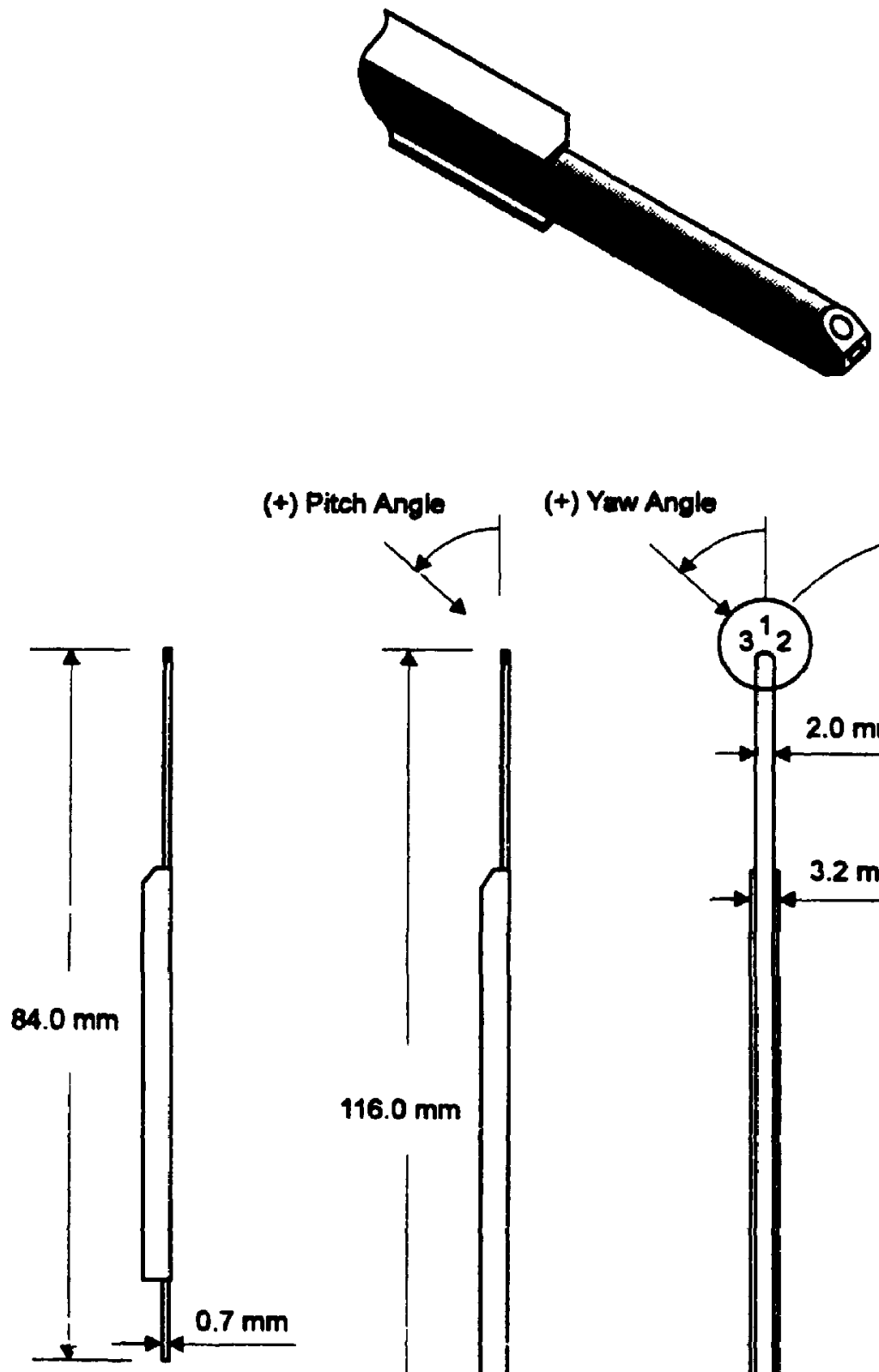

(+) Pitch Angle

(+) Yaw Angle

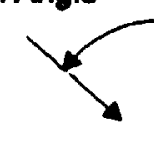

Inlet Probe

(t) Yam Anglo

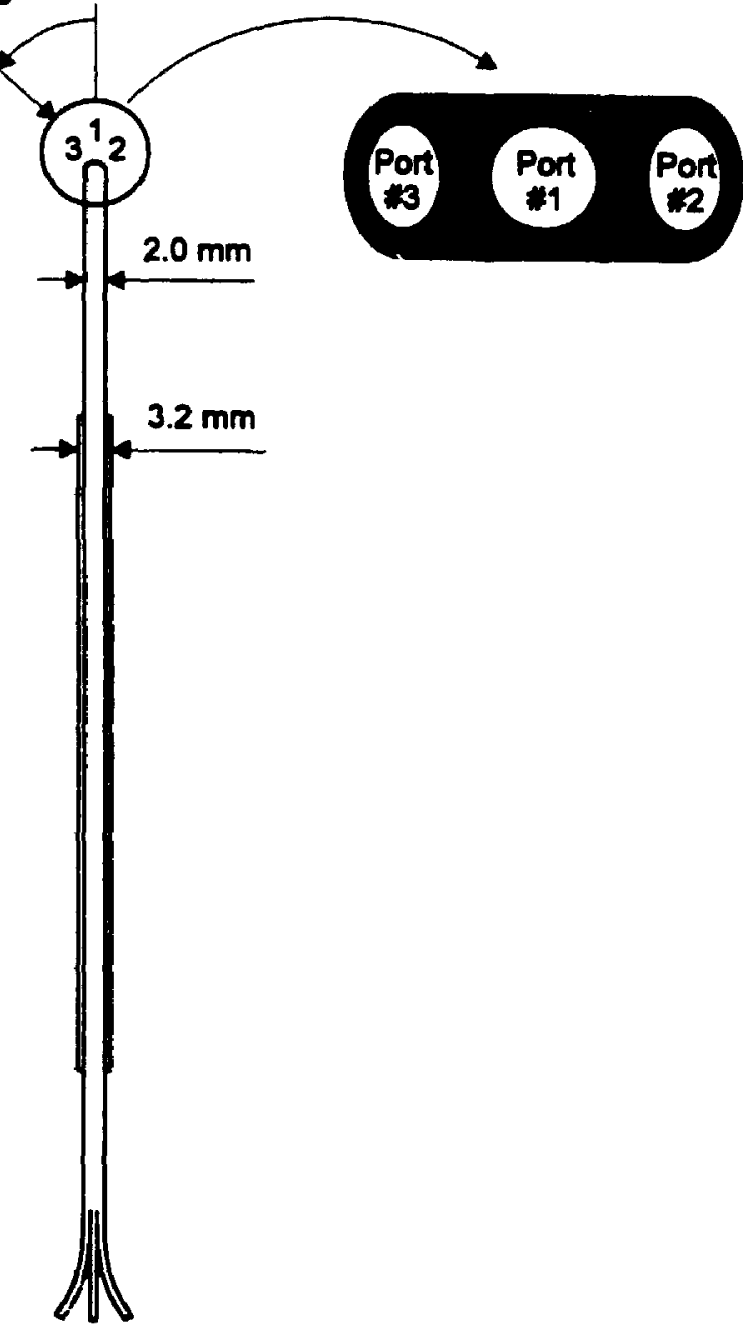

QutlatProbe

Figure 3.5: Three-hole pressure probe geometry. 


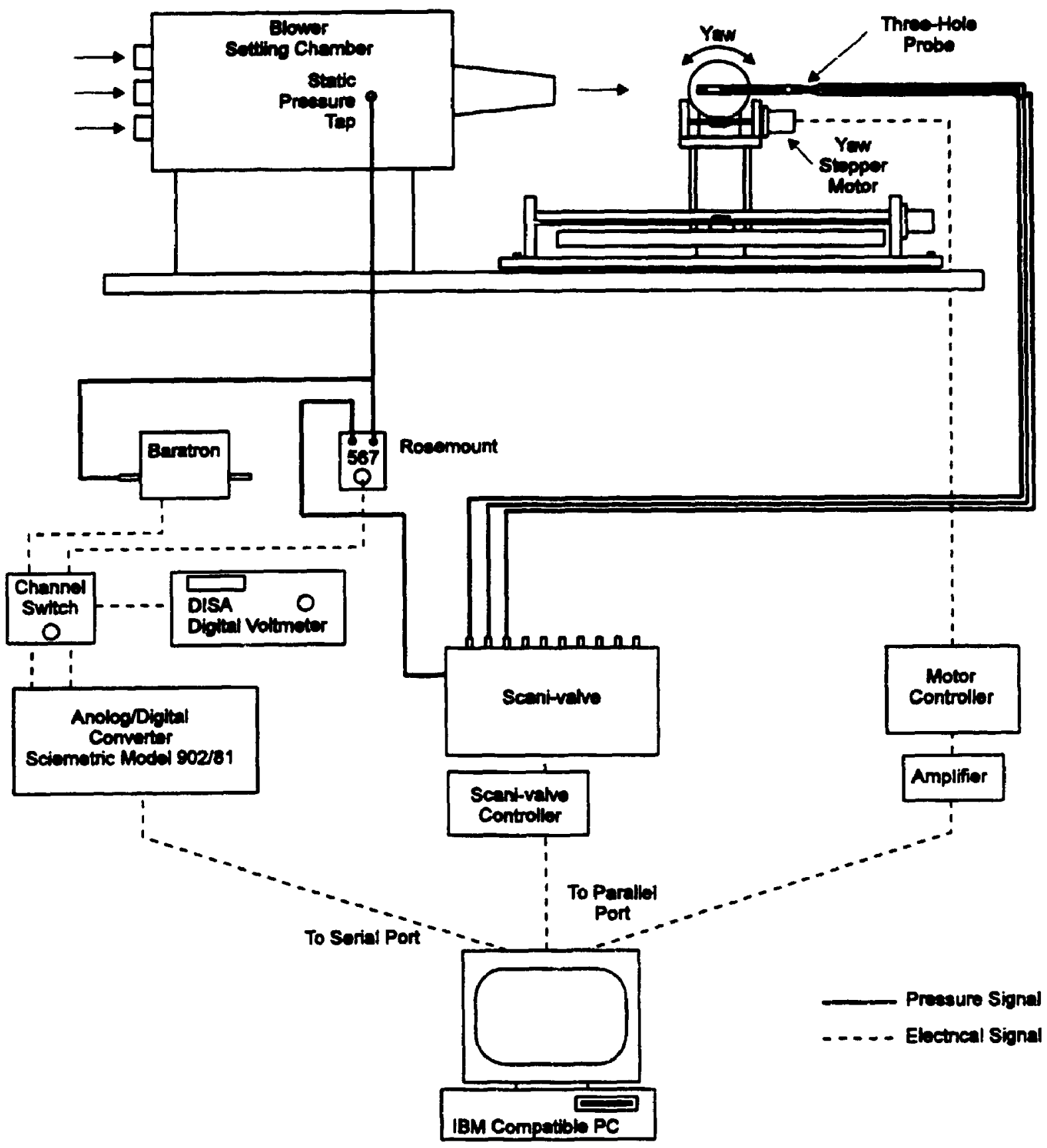

Figure 3.6: Data acquisition setup for calibration apparatus. 
to the cascade test section inlet or outlet velocities depending on which probe was being calibrated. The probe yaw angle was varied from $-10^{\circ}$ to $+10^{\circ}$ in $0.5^{\circ}$ increments, with a fixed pitch angle. At each increment the pressures of all three probe ports were recorded. The measured pressures were then used to obtain calibration curves for total pressure, velocity, flow angle and static and dynamic pressure. The calibration coefficients and resulting curves are shown in Appendix C. For a detailed description of the calibration procedure see Rodger (1992).

The waiting and sampling time required to achieve a good average for the signal was investigated. The use of a scani-valve impedes the efficiency of the data acquisition system. When the scani-valve switches from one input to the next a certain amount of time must be allotted to ensure that the pressure in the pneumatic circuit has reached the new value. A worst case scenario was investigated in order to resolve the necessary waiting time and sampling time. The outlet three-hole probe was installed in the calibration apparatus at a yaw angle of $+10^{\circ}$. At a high probe yaw angle the scani-valve would have to step from a high positive to a high negative value. The cumulative averages of the transducer output voltages were plotted versus time for all three ports. Results showed that a 20 second waiting period followed by a 20 second sampling period is conservative for the probe. The acquisition system sampling rate is approximately 5 samples per second. The pressure signals are then averaged over the $\mathbf{2 0}$ second sampling period, reduced to pressure values, non-dimensionalized by reference values, and stored in the data files on the microcomputer. For example, during the 20 second sampling period roughly 110 samples are taken from each of the two transaiucers. These samples arc thes: non-dimensionlized to form roughiy $11 \mathrm{C}$. 10 -dimensional pressure coefticients which are then averaged to give one value for the sampling period.

The TYGON tubing used to connect the three-hole probe to the scani-valve during the waiting and sampling time tests was the same tubing used for the cascade measurements. Therefore, the required waiting and sampling time determined were also applied to the wind tunnel testing. 


\subsubsection{Accuracy of the Three-Hole Probes}

The accuracy of the three-hole probe measurements was investigated by Yaras (1987) and Tremblay (1989).

Yaras (1987) investigated Reynolds number effects on the accuracy of the probe measurements. He concluded that for small flow yaw angles (within $-30^{\circ}$ to $+30^{\circ}$ range) the error in measured flow angle was less than $0.5^{\circ}$ for a probe tip Reynolds number in the range of 2000 to $\mathbf{3 7 0 0}$. The range of flow angles and Reynolds numbers used in the present study are well within the acceptable ranges (i.e. yaw angles $\pm 10^{\circ}$, probe Reynolds numbers from 2100 to 3100 ).

An accuracy assessment on the probe was also performed by Tremblay (1989). Using the uniform jet flow from the calibration apparatus he found that flow angles were accurate to within $\pm 0.5^{\circ}$ in the $-10^{\circ}$ to $+10^{\circ}$ range and the error in the measured dy samic pressure was less than $1 \%$ of the actual local dynamic pressure. He also determined that there was an additional error introduced in the flow angle when measuring in a nonuniform flow, such as in the wake of a blade. Tremblay (1989) investigating a very similar cascade geometry, namely $\mathrm{CC} 2$, found the error in flow angle in the wake was estimated to be within $\pm 1 \%$. Using a pitot tube in the blade wake he found that the error in dynamic pressure was less than $3 \%$. He concluded that there was a $\pm 5 \%$ error on the mass average total-pressure-loss coefficient. In the pifsint research attempts were made at reducing this error by introducing the new smaller outlet probe design. The probe was designed using smaller tubing than previously used (shown in Appendix F). Unfortunately, attempts at calibrating the new probe failed. At high yaw angles the low pressure at one of the probe ports could not be measured accurately. Therefore, the new probe design was abandoned and the outlet probe shown in Figure 3.5 was used for all downstream rneasurements. 


\subsection{Measurement of Cascade Performance}

\subsubsection{Operating Point}

The tunnel operating point is established in terms of the Reynolds number. The present experiments have been performed at a Reynolds number of 300,000 which is the same Reynolds number used by previous investigators (eg., Abbott (1993), Whitehouse (1993), Rodger (1992)). The Reynolds number is based on the blade chord, inlet centerline velocity, atmospheric pressure and stagnation temperature inside the contraction. This differs slightly from past investigations where the room temperature of the laboratory was used to evaluate the Reynolds number properties. During the early stages of the present study, investigations showed that the temperature in the test section could be up to $10^{\circ} \mathrm{C}$ higher than the temperature of the room. Therefore, modifications were made to the wind tunnel contraction to allow the temperature to be monitored from within. To obtain a Reynolds number of 300,000 in the wind tunnel test section, the fan speed controller is adjusted until the dynamic pressure measured at the inlet corresponds to the necessary velocity (approximately $30 \mathrm{~m} / \mathrm{s}$ ).

\subsubsection{Inlet Measurements}

Before loss measurements can be made it is first necessary to establish a uniform flow across the inlet of the cascade; that is, the incoming flow at each blade ' hould be parallel to the axial direction. Several control surfaces on the test section, shown in Figure 3.7, are used to adjust the flow. The primary control surfaces affecting the inlet flow are the top and bottom flaps. The inlet flow angle is measured using the short threehole pressure probe. The probe is mounted at midspan in a holder socket which is attached to the end of a mounting rod. The mounting rod is attached to a traversing gear which allows the probe to be traversed in a pitchwise direction $60 \%$ axial chord upstream 


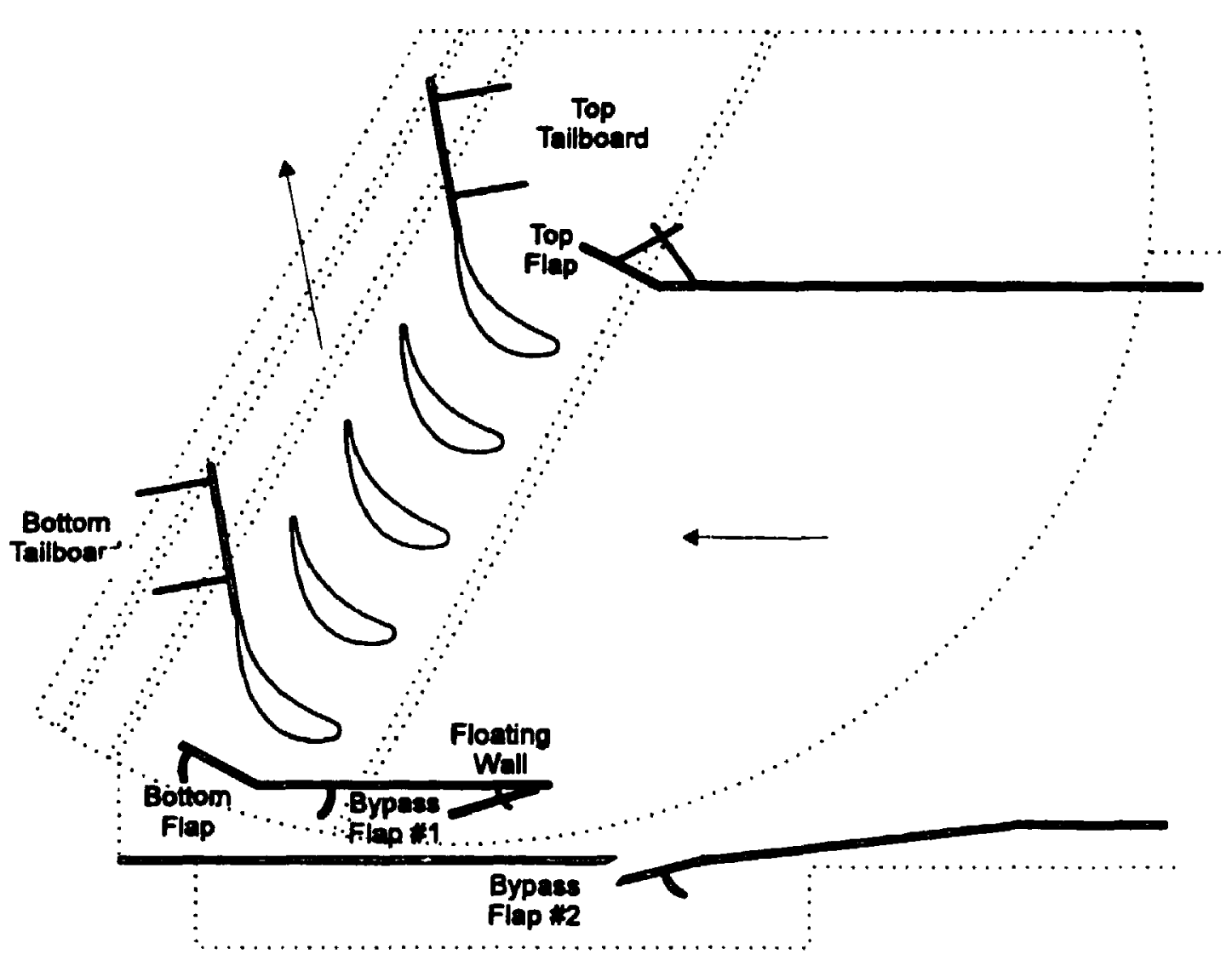

Figure 3.7: Test section control surfaces.

of the leading edge plane. A bubble level is used to align the probe at an angle of $0^{\circ}$ to the horizontal with an estimated alignment accuracy of $\pm 0.5^{\circ}$.

The control surfaces are initially set to values established by previous investigators. A very coarse traverse of the inlet flow is carried out to get an overall impression of the flow. The control surfaces at the top and bottom are then adjusted and the flow is re-measured. This procedure is repeated until the inlet flow angle is within $\pm .5^{\circ}$ of zero and does not vary more than $0.5^{\circ}$ across the front of the two central blade passages. Once this criterion is met a fine traverse of the inlet plane is performed. Figure 3.8 shows a typical inlet flow result after the adjustment of the control surfaces has been completed. 


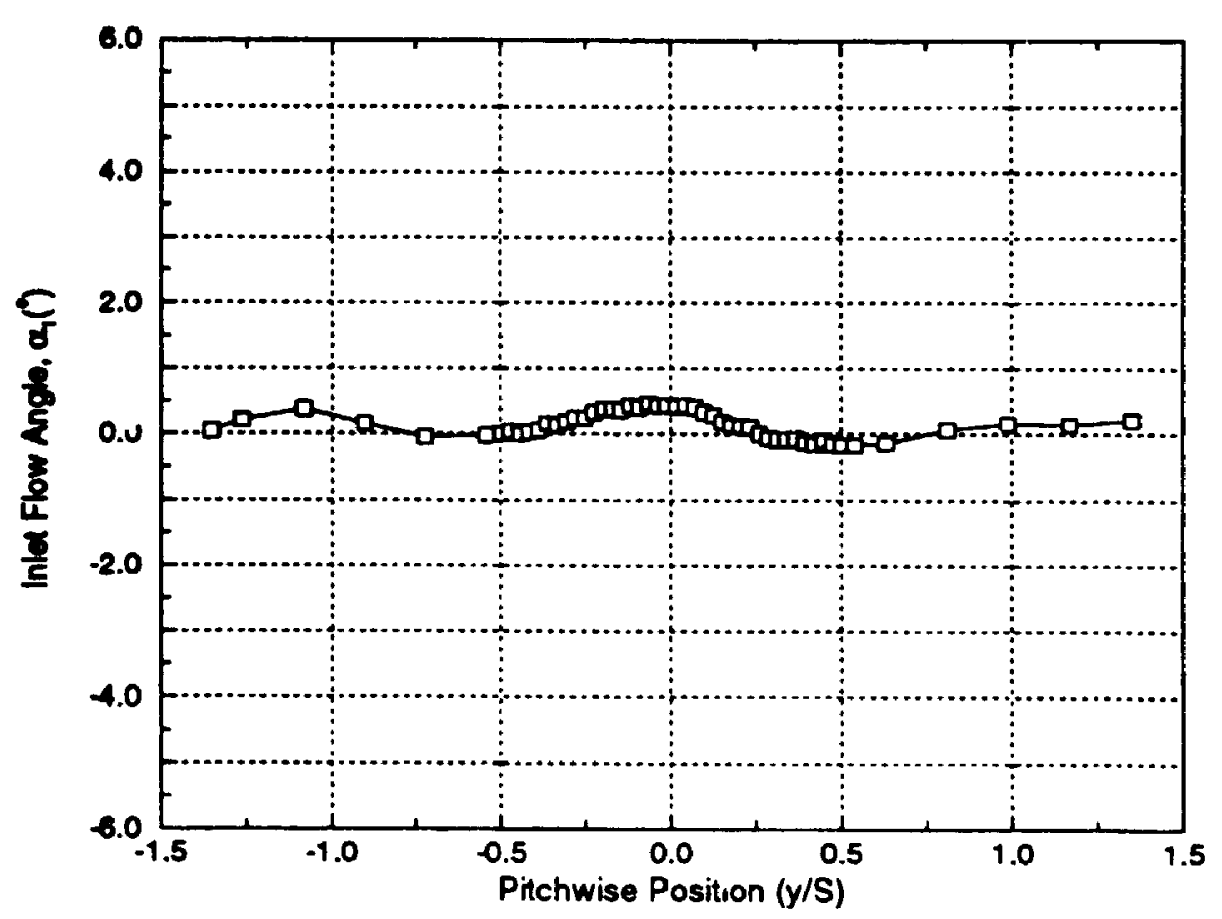

Figure 3.8: Typical inlet flow angle distribution at design incidence.

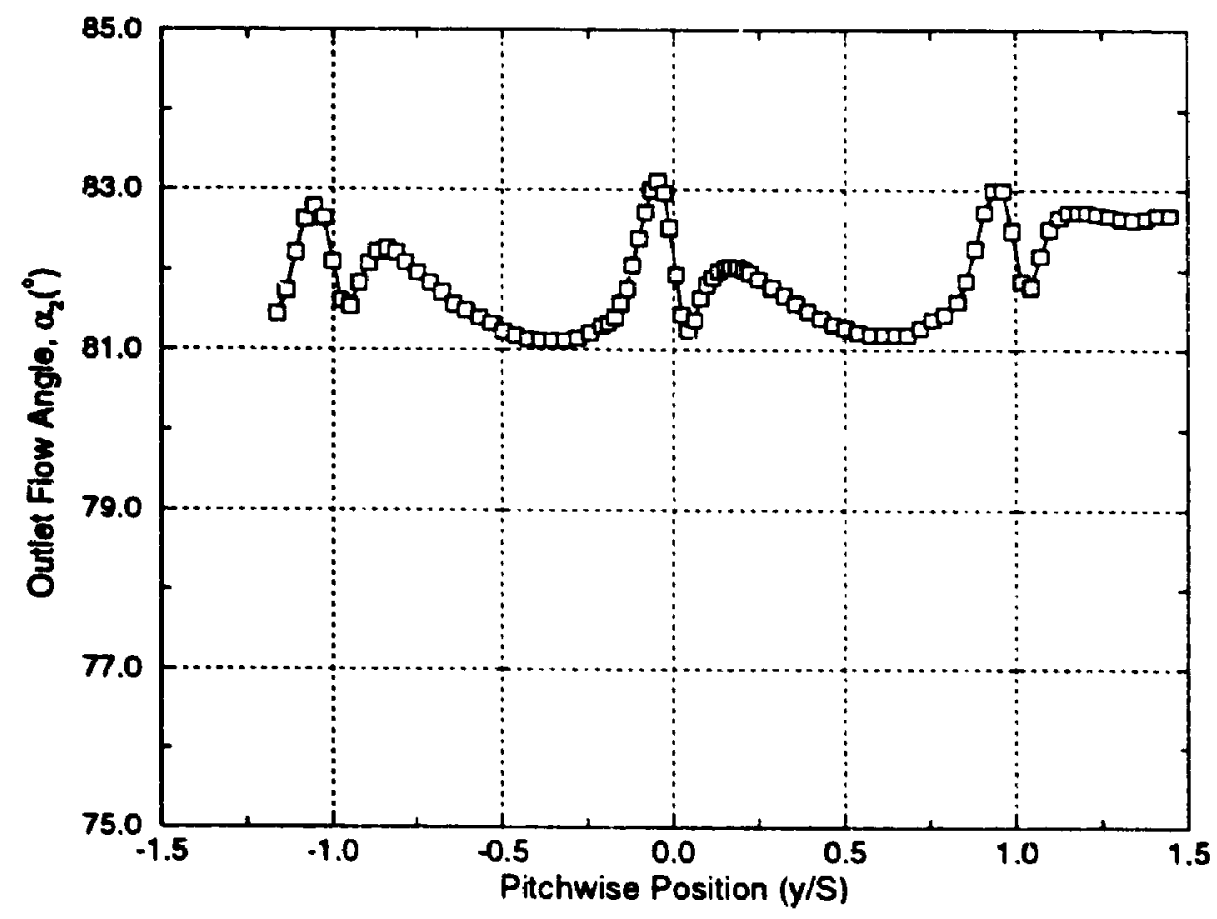

Figure 3.9: Typical outlet flow angle distribution at design incidence. 


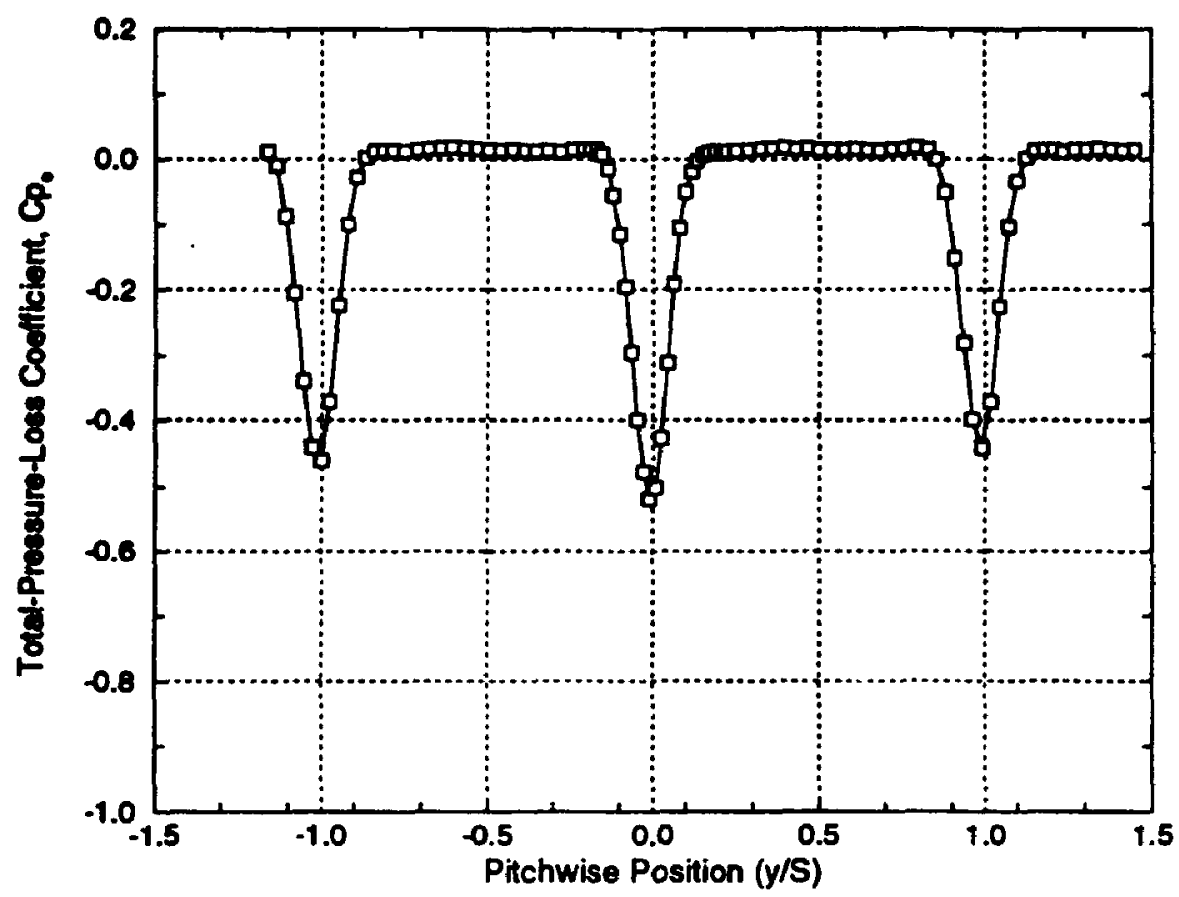

Figure 3.10: Typical outlet total-pressure-loss coefficient distribution at design incidence.

\subsubsection{Outlet Flow Uniformity and Periodicity}

Once a uniform inlet flow has been achieved, the inlet probe is removed and the traverse slot machined in the endplate is taped over. The outlet probe is then mounted aligned with the nominal flow direction at midspan in a holder socket which is attached to a mounting rod. The mounting rod extends back through a slot in the endwall where it is attached to a traversing gear. The traversing gear has two degrees of freedom allowing the probe to make spanwise and pitchwise measurements. The probe was roughly aligned with the flow using a bubble level with an attached adjustable protractor.

Before performing outlet loss measurements, the flow and losses must be periodic so that an infinite cascede is simulated. Use of the control surfaces in an iterative process enables the flow to be adjusted so that it leaves with periodicity. A coarse pitchwise 
traverse $40 \%$ axial chord downstream of the trailing edge is performed with the three-hole probe. The outflow angle and total pressure are observed and plotted. If the flow angles between two adjacent blade passages are not similar and or the wake geometries behind three adjacent blades are not similar the position of the tailboards can be adjusted and the flow re-measured. Adjustment of the tailboards may affect conditions at the inlet so the flow uniformity at the inlet also has to be re-measured. This procedure is repeated until the outlet flow angle at $y / S= \pm 0.5$ is periodic within $1^{\circ}$ and the wake shape of the three blades is similar. Once this is achieved a fine traverse of the outlet plane is performed. Typical results of outlet flow angle and total-pressure-loss coefficient are shown in Figure 3.9 and Figure 3.10 respectively.

Initial tailboard settings used in the present research can be found in Appendix D.

\subsubsection{Flow Two-Dimensionality and Axial Velocity Ratio}

The measurements to be dissussed in Chapter $\mathbf{5}$ are presented as two-dimensional losses. This can only be considered valid if the flow at the location being measured can be considered two-dimensional. Rodger (1992) proposed that one could infer the existence of two-dimensional flow if the following three conditions are met: the axial velocity ratio (AVR) is equal to 1.0, a spanwise region of uniform total pressure in the middle of the cascade outlet exists, and a spanwise region of uniform mass-average velocity and flow angle at the outlet of the cascade exists.

The axial velocity ratio is a common measure of the flow acceleration through the cascade passage; it is defined as the ratio of the trailing edge axial velocity to the leading edge axial velocity (see Figure 3.11). The velocities obtained at the inlet and outlet plane are mass averaged over one pitch. Ideally, the mass averaged velocities would be evaluated at the blade leading edge and trailing edge, but due to equipment constraints 
this is impossible. A linear interpolation is used to determine the values at the leading edge and the trailing edge.

By adjusting the two endplates the flow can be accelerated or decelerated through the cascade passage thereby increasing or decreasing the axial velocity ratio. Adjustment of the endplates will affect the conditions at the inlet and outlet of the cascade thereby requiring the inlet and otitlet uniformity to be retested. This procedure was repeated until an AVR $=1.0 \pm 0.01$ was achieved.

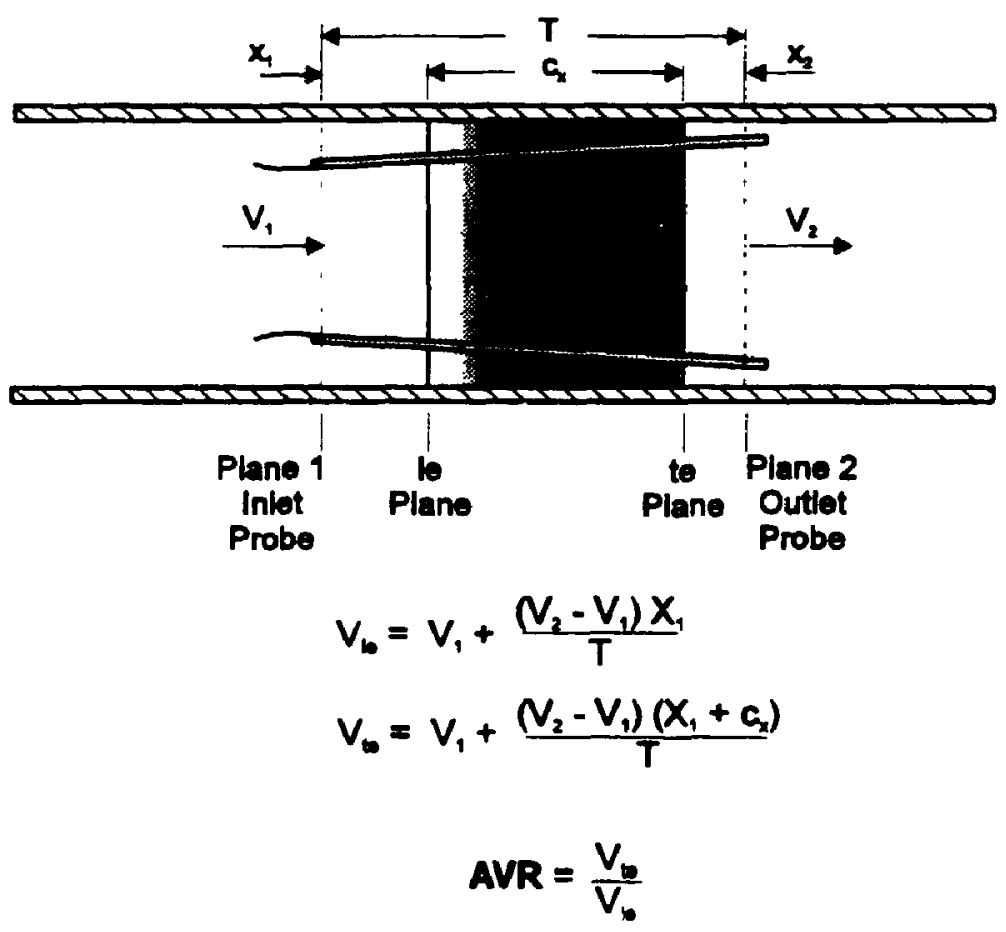

Figure 3.11: Schematic top view of the cascade and endplates presented with the interpolation scheme used to determine the axial velncity ratio. 
A spanwise region of uniform total pressure, mass-average velocity and flow angle at the outlet of the cascade are also necessary conditions for two-dimensional flow. However several studies have demonstrated that substantial regions of uniform flow exists for the tested cassade configuration. Rodger (1992) and Whitehouse (1993) investigated the spanwise total-pressure-loss distribution for smooth blades. The midspan losses were found to be uniform in the midspan region. Abbott (1993) also investigated the spanwise distribution of the outlet flow velocity, angle and total pressure with roughness applied to the blade surface at design and off-design incidence angles. He found that a spanwise extent of uniformity to axial chord ratio of $15 \%$ exists at the cascade outlet for a worst case scenario (i.e. at high positive incidences). During this investigation a few checks were made to confirm the earlier results regarding spanwise unifonnity.

\subsubsection{Data Reduction}

After the inlet and outlet flow uniformity had been firmly established and an axial velocity ratio near unity confirmed, proper measurements could be made. Total-pressure loss and flow deviation could be calculated readily from the data files created during the outlet traverse. The data files contain pressure coefficients for each probe port at each traverse station. Using the probe calibration coefficients established in Section 3.2.2.1 the pressure coefficients are converted to flow angles, dynamic pressures, static pressures, and total pressures at every traverse station. A total-pressure-loss coefficient is calculated at each traversing station as follows:

$$
C p_{0}=\frac{P_{02}-P_{0 \text { ref }}}{q_{\text {ref }}}
$$

where: $\quad P_{\text {oref }}:$ total pressure at reference plane

$P_{02} \quad:$ total pressure at outlet measurement plane

$q_{\text {ref }}:$ dynamic pressure at reference plane 
The inlet dynamic and total pressures are measured using a pitot-static system shown in Figure 3.4 or 3.12 at the reference plane, not at the inlet of the cascade. This assumes that the total pressure measured at the reference plane is equal to the total pressure at the cascade inlet. Abbott (1993) investigated this and found that the pressure loss from the reference plane to the cascade inlet is indeed negligible.

A modified version of the program THP, written by Whitehouse (1993) and modified by Abbott (1993) was used to process the data. The total-pressure loss, flow angle, and axial velocity are mass averaged quantities at midspan over one blade spacing, $S$. The total-pressure-loss coefficient is defined as follows:

$$
Y_{m}=\frac{P_{\text {orf }}-P_{02}}{q_{2}}
$$

where $q_{2}$ is the mass-averaged dynamic pressure measured at the plane of the probe downstream of the cascade. The coefficient is evaluated as follows:

$$
Y_{m}=\left(\frac{1}{\dot{m}} \int_{0}^{S} C p_{o} d m\right)\left(\frac{q_{\text {rff }}}{q_{2}}\right)+\left(1-2 \frac{\int_{0}^{1} V_{2}^{2} d(y / S)}{\left(\int_{0}^{1} V_{2} d(y / S)\right)^{2}}+\frac{\int_{0}^{1} V_{2}^{3} d(y / S)}{\left(\int_{0}^{1} V_{2} d(y / S)\right)^{3}}\right)
$$

The first term is the mass-averaged total-pressure-loss coefficient calculated by integrating the area under the pressure loss coefficient curve $\left(C p_{o}\right)$ and the second term accounts for the downstream mixing loss. The mixing loss term accounts for the additional loss which occurs as the wakes mix out to a uniforn flow at some distance downstream. It assumes constant-area mixing. The derivation of the mixing loss component is given in Goobie (1989).

The flow deviation is calculated by subtracting the mass-averaged outlet flow angle calculated by the data reduction program from the blade exit metal angle. 


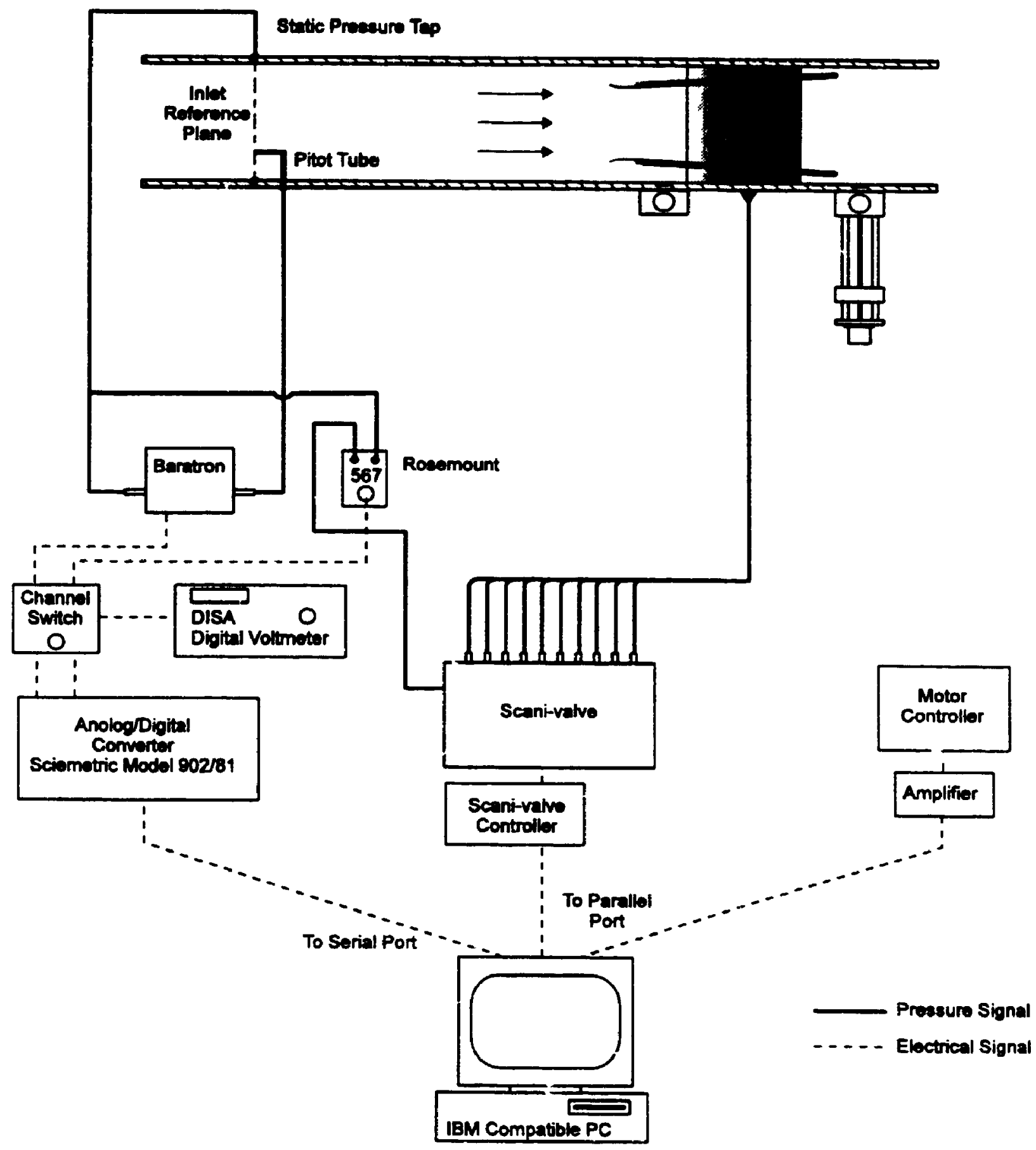

Figure 3.12: Data acquisition setup for static blade measurements. 


\subsubsection{Blade Static Pressure Measurements}

The blade static pressure measurements were performed ising the instrumented blade as described in Section 3.1.2. Figure 3.12 shows the data acquisition system arrangement. The static pressure measurements were made immediately following the profile loss and flow deflection measurements, without altering the flap settings for a specific test case. This ensured that an axial velocity ratio close to unity was still present through the cascade. Pressure distributions were obtained with and without surface roughness for flows at $0^{\circ}, \pm 10^{\circ}$, and $+15^{\circ}$ effective incidence. Each static tap is sampled in the same manner used for the three-hole probe measurements. A conservative waiting time of $\mathbf{3 0}$ seconds and sampling time of $\mathbf{3 0}$ seconds at $\mathbf{5}$ samples per second was used. The results are stored as pressure coefficients based on the measured value and the upstream dynamic and static pressures as follows:

$$
C_{p \text { meas }}=\frac{\left(P_{s}-P_{r f f}\right)}{q_{\text {ref }}}
$$

The reference values used in the above equation are located far upstream of the cascade leading edge plane (see Figure 3.12); thus they are not exactly representative of the true inlet conditions of the cascade. Inlet uniformity tests are made at $60 \%$ chord upstream of the leading edge and these data are also normalized by upstream conditions $P_{\text {ref }}$ and $q_{\text {ref }}$ Therefore, using the followirg simple relationship, the proper value of $C p$ can be calculated:

$$
\begin{aligned}
C_{p} & =\frac{\left(P_{s}-P_{1}\right)}{q_{1}} \\
& =\left(\frac{P_{s}-P_{\text {ref }}}{q_{\text {ref }}}-\frac{P_{1}-P_{\text {ref }}}{q_{\text {ref }}}\right) \frac{q_{\text {ref }}}{q_{1}} \\
& =\left(C_{p \text { meas }}-\frac{P_{1}-P_{\text {ref }}}{q_{\text {ref }}}\right) \frac{q_{\text {ref }}}{q_{1}}
\end{aligned}
$$


The value of $P_{I}$ and $q_{I} / q_{\text {ref }}$ are extracted from the corresponding inlet uniformity tests. Repeatability tests made on the blade pressure measurements show errors in static pressure coefficients of less than one percent on the value of $C_{p}$.

\subsubsection{Boundary Layer Transition Measurements}

A small pitot tube, attached by TYGON tubing to a stethoscope, was used to determine the approximate point at which the laminar boundary layer begins transition to a turbulent boundary layer. The outside diameter of the pitot tube tip was $1 \mathrm{~mm}$ and the length of the tube was approximately $35 \mathrm{~mm}$. A second tube was placed in a region known to be laminar. This enabled the listener to differentiate between turbulent and laminar flow. The pitot tube was essentially aligned with the flow direction and placed on the blade surface initially near the leading edge. The pitot tube was then traversed slowly along the blade surface toward the trailing edge. Near the point where transition begins, the fluctuations caused by the turbulence become larger and therefore more distinguishable to the ear. The approximate point for the onset of transition was marked on the blade surface. Repeatability tests demonstrated that this point could be determined with an accuracy of $\pm 5 \mathrm{~mm}$.

\subsection{Roughness Selection and Application}

\subsubsection{Roughness Selection Procedure}

Three different sizes of sand grains were used in this study. Their relative sizes are shown schematically in Figure 3.13. Defined as large, medium and small, their approximate nominal grain size, $k$, were $1020 \mu \mathrm{m}, 722 \mu \mathrm{m}$ and $337.5 \mu \mathrm{m}$ respectively. 


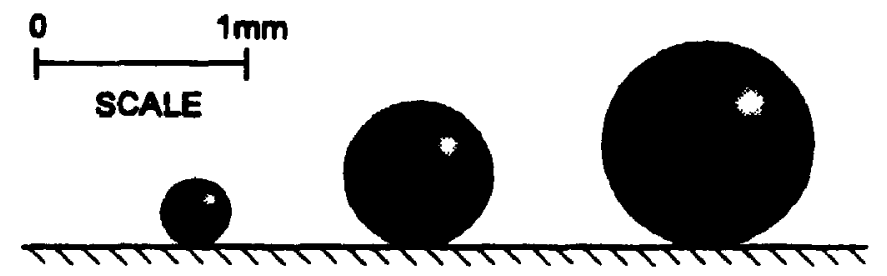

Figure 3.13: Relative size of sand grains.

Table 3.1: Sand Grain Sizes (data reproduced from Abbott (1993)).

\begin{tabular}{|c|c|c|c|c|c|}
\hline $\begin{array}{l}\text { Grade \# of } \\
\text { Catching } \\
\text { Screen }\end{array}$ & $\begin{array}{l}\text { Mesh Size of } \\
\text { Passed Screen } \\
\text { (pm) }\end{array}$ & $\begin{array}{c}\text { Mesh Size of } \\
\text { Catching Screen } \\
(\mu \mathrm{m})\end{array}$ & $\begin{array}{l}\text { Nominal Sand } \\
\text { Grain Size, k } \\
\text { ( } \mu \mathrm{m})\end{array}$ & $\begin{array}{c}\text { Non-Dimensional } \\
\text { Sand Grain Size } \\
\text { k/c }\end{array}$ & Classification \\
\hline $\begin{array}{c}20 \\
\text { (Silica) }\end{array}$ & 1190 & 850 & 1020 & 0.00628 & Large \\
\hline $\begin{array}{c}30 \\
\text { (Silica) }\end{array}$ & 850 & 594 & 722 & 0.00445 & Medium \\
\hline $\begin{array}{c}60 \\
\text { (Beach) }\end{array}$ & 425 & 250 & 337.5 & 0.00208 & Small \\
\hline
\end{tabular}


Silica sand made up the medium and large grain samples whereas treach sand made up the small grain samples. The sand was sifted using screens and a vibrating platform. The screens were stacked such that the top screen (passed screen) had mesh openings slightly larger than the desired nominal size, and the second screen (carching screen) had mesh openings slightly less than the desired nominal size. The nominal grain size of the sand is taken as the average of the mesh opening size of the screen or which the sand has been caught and that of the last screen through which the sand has passed. The types of sand collected, the approximate grain sizes, and the screen sizes used are listed in Table 3.1.

\subsubsection{Roughness Application Equipment}

The roughness application apparatus was designed and developed by Abbott (1993) and is shown in Figure 3.14. The apparatus includes a sand hopper, parallel tracks, a wheeled cart and a small AC electric motor.

The sand hopper consists of a wooden frame, a sand container and two aluminum plates. One of the aluminum plates is fixed to the wooden frame whereas the other is clamped to the frame with C-clamps such that a specific gap is maintained between the two plates. The size of the gap is determined by the size of the sand being used. A rectangular hole in the bottom of the container allows the sand to flow through the gap between the aluminum plates, onto the surface of the cascade blade.

The wooden track is 2.3 meters long and 0.26 meters wide. A cart 0.3 meters wide and 0.24 meters long is pulled along the track by a string, which is wound on a pulley attached to the AC electric motor. A variac (Superior Electric Type 3PN116B) was used to control the speed of rotation of the AC electric motor and hence the speed of the cart. 


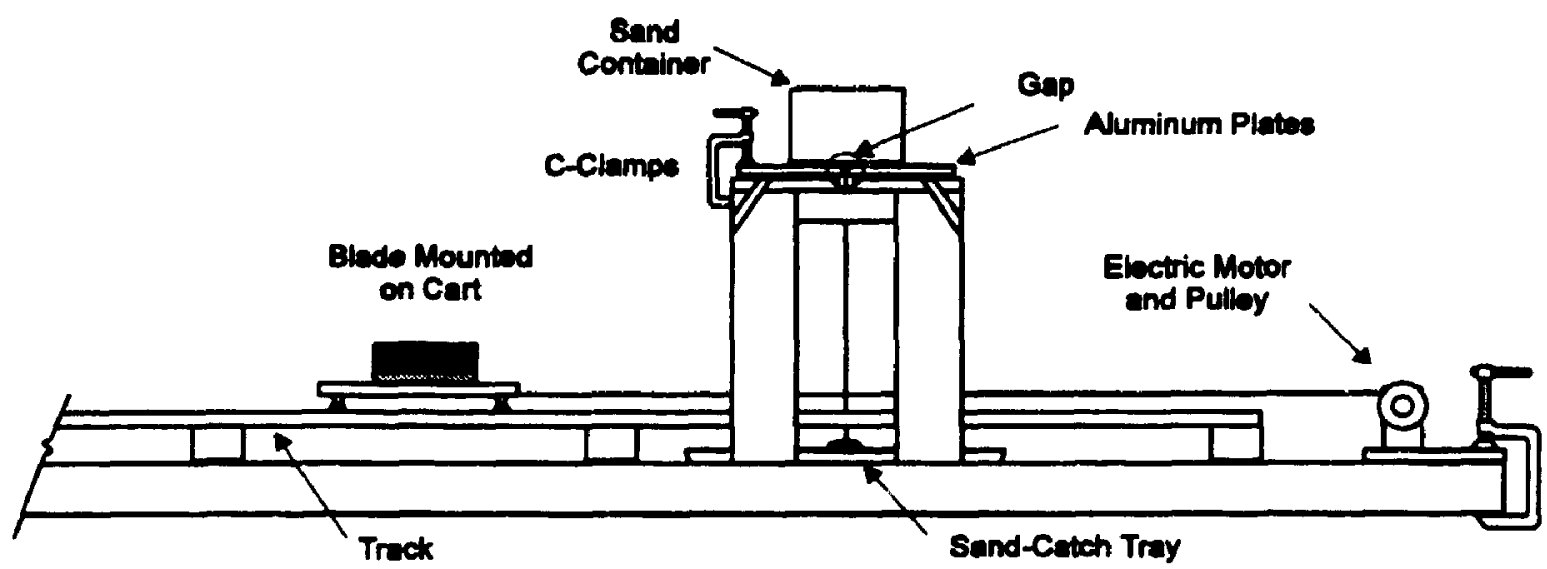

Figure 3.14: Schematic of roughness application apparatus.

The sand is adhered to the surface of the blade using a vinyl strippable coating (Crown Vinyl Strippable Coating product \#68040). Abbott (1993), using a Digital Scanning Electron Microscope investigated the effects of using the vinyl strippable coating. He determined that the adhesive did not change the apparent shape of the sand grains and that the thickness of the adhesive was relatively small in comparison to the sand grains.

\subsubsection{Roughness Application Procedure}

A uniform sand grain distribution with the capability for attaining a large range of the spacing between grains was desired. This was accomplished by adjusting the variac setting (and hence the velocity of the cart) and the gap setting of the hopper.

The gap size between the aluminum plates was set to a predetermined value (determined by trial and error) corresponding to the size of sand grains used. The 
container filled with sand of a uniform size was placed on top of the hopper with a sheet of paper covering the gap between the aluminum plates. The area of the blade to be covered with said was outlined with masking tape. The blade was secured to the cart and placed at the far end of the track. A coating of the vinyl strippable paint was applied to the blade and the sheet of paper between the sand container and aluminum plates was removed to start the sand flowing. The electric motor was started and the cart with the blade atop was pulled down the track. As the cart passed under the sand hopper the sand fell onto the adhesive and remained attached. When the cart reached the end of the track, the cart was arrested and the masking tape was removed. The vinyl coating completely dries within 5 minutes. If the roughness on the blade was not uniformly distributed, the roughness was removed and the application process was repeated. The gap size and the variac setting were recorded. The blade was then removed from the cart and subjected to a more thorough examination using digital image processing (Section 3.4.4). A value of approximately 10 for the spacing parameter, $\lambda$, was desired. If the roughness distribution was within \pm 1 the sample was preserved; if not the roughness was removed and the gap size or cart velocity varied and the application process repeated.

\subsubsection{Examination of the Roughness Distribution}

Digital image processing was used to evaluate the spacing parameter of the sample. A charged coupled device (CCD) camera mounted on a support stand is focused on the sample surface. The camera is connected to an IBM compatible personal computer. A software package, Optimas, is used to digitize the sample surface to a 640 $x 480,8$ bit grey scale image. Using thresholding techniques the individual sand grains were identified and separated from the background sample surface. Knowing the average particle size and the number of individual sand grains, the spacing parameter was then easily determined using the following relation: 


$$
\lambda=\frac{A_{T}}{A_{F}}
$$

where $A_{T}$ is the total area and $A_{F}$ is average frontal area of one particle multiplied by the number of particles within the area $A_{T}$.

Prior to digitizing the sample, a scale was laid on top of the sample surface in order to calibrate the camera. Once calibrated, eight different surface areas were used to calculate an average spacing parameter per blade. Appendix E contains a figure showing approximately the eight different areas of the roughness samples investigated and a table with the calculated spacing parameters for the various roughness applications. 


\section{Chapter 4}

\section{COMPUTATIONAL ANALYSIS}

The experimental cascade was modelled computationally using a viscous/inviscid interaction approach. The objective was to investigate the ability of the computational method to predict the pressure distribution, total pressure loss and flow deflection for smooth and rough surface cascades at design and off-design angles. This chapter describes how the computational method was used in the present research. The first section briefly outlines the computational method. The following sections describe how the experimental cascade was modelled computationally and describe the post-processing used to compare the computational predictions with the experimental results. The final section highlights some of the potential sources of error in the computational method.

\subsection{Outline of the Computational Method}

Inviscid flow computations for the experimental cascade were done using a vortex panel method developed at Carleton University. Vorticity varies linearly along the panels and is continuous at the junction points between adjacent panels, schematically illustrated in Figure 4.1. Linearly varying source distributions are also used on the panels to model boundary layer displacement effects. The numerical formulation of the panel method is not provided here; details are available for isolated airfoils in Kind (1973) and Bittle (1985), and for profiles in cascade in Kind (1992). The approach taken is to compute the potential flow over a finite array of identical profiles; the 'primary' profile is in the middle of the array and the other profiles are referred to as 'companion' profiles. As 
shown in Figure 4.2, the companion profiles are represented by panels having the same geometric configuration and the same vorticity and source distribution as those representing the primary profile. The boundary condition of zero-velocity normal to the profile is applied at the control points on the primary profile to obtain a set of equations that is solved for the unknown vorticity values at the junction points.

The pressure distribution obtained from the inviscid flow computations provides the free-stream velocity distributions for the computation of the boundary-layer development. The upper and lower surface computations begin at the leading edge stagnation point. Thwaites' (1949) integral method was used to predict the growth of the

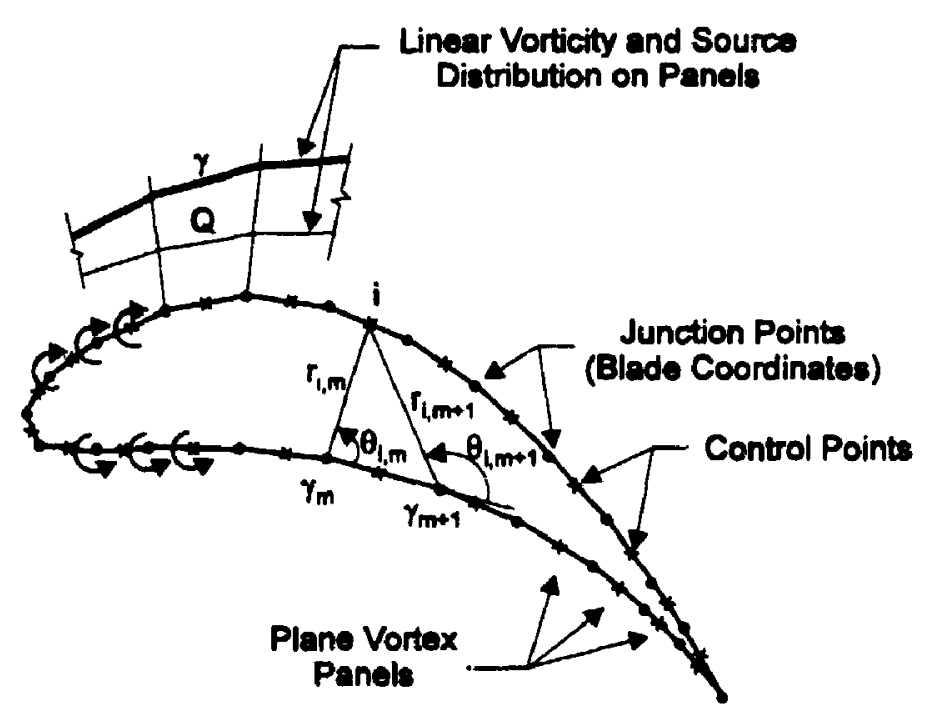

Figure 4.1: Schematic illustration of the inviscid computational model. 


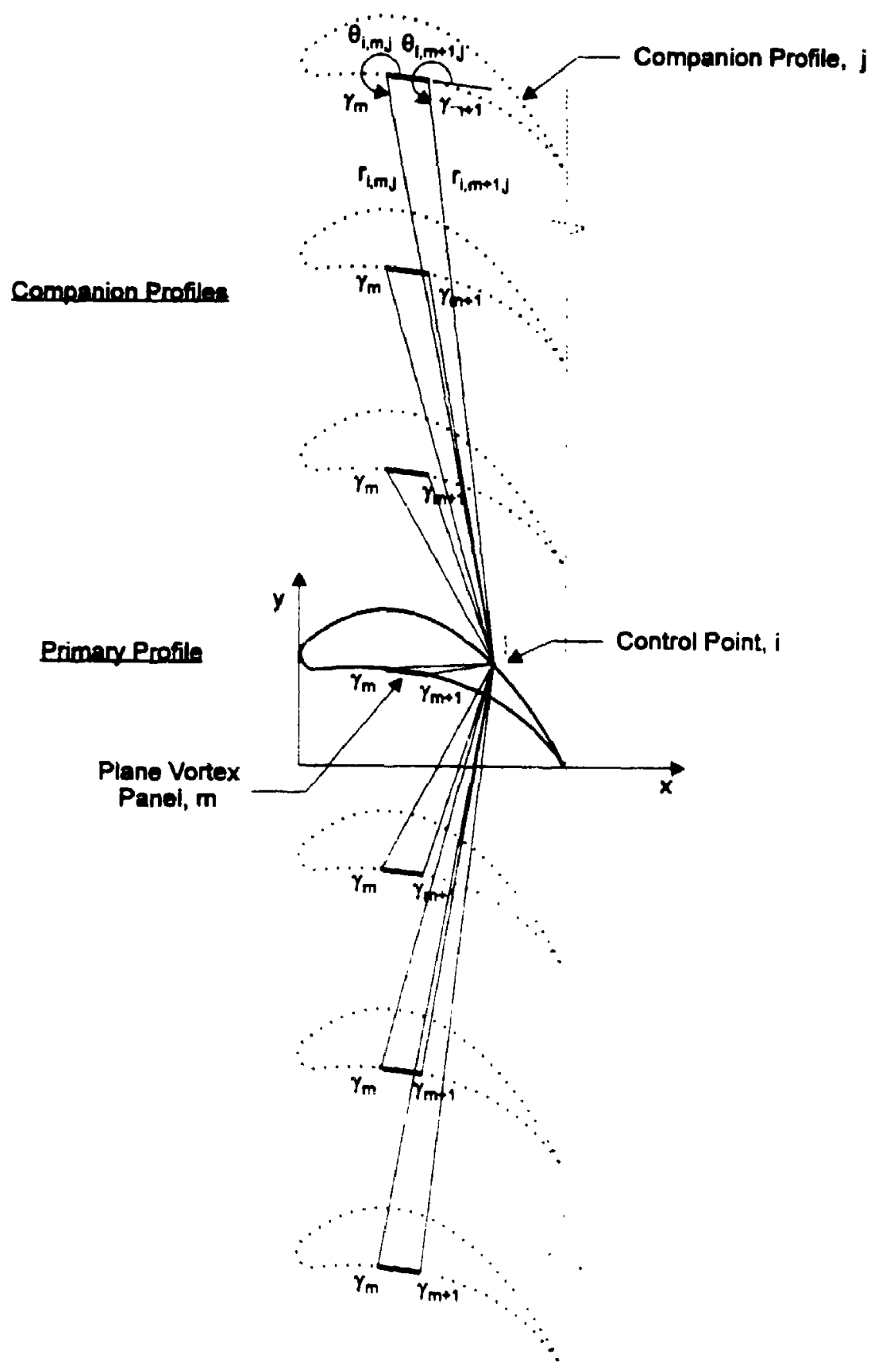

Figure 4.2: Schematic illustration of a finite array of profiles. 
laminar boundary layer and the $H-R_{x}$ method developed by Wazzan et al. (1981) was used on smooth upper and lower surfaces to predict transition. Transition on rough surfaces was predicted if the local dimensionless roughness height was greater than 20 , i.e:

$$
\left|\frac{U_{\tau} k}{v}\right|_{\text {Trans }} \geq 20
$$

where $U_{T}$ is the local shear velocity, $k$ is the roughness height and $v$ is the kinematic viscosity. Turbulent boundary-layer parameters were calculated by using the improved entrainment method developed by Head and Patel (1968), coupled with Dvorak's (1969) skin-f: . ion law for rough surfaces. The improved entrainment method was shown by Head and Patel to give substantially better results than Head's (1958) original entrainment method for a wide variety of boundary layer flows. Dvorak's skin friction law recognizes the effects of both roughness height and roughness spacing. The boundiry-layer computations provided the displacement thickness required to determine the st ngth of the surface-source distribution in the inviscid flow computations. The required junction point source strength is given by:

$$
Q=\frac{d}{d S}\left(U_{E^{*}}\right)
$$

where $S$ denotes the arc iength, $U_{E}$ is the local inviscid flow velocity and $\delta^{*}$ is the local boundary layer displacement thickness. The source strengths during the second and subsequent passes through the panel-method computation are thus known and appear on the right hand side of the aforementioned boundary condition equations. 


\subsection{Application of the Computational Method}

\subsubsection{Modified Blade Geometry}

The blunt trailing edge of the actual blade was approximated by a sharp trailing edge as shown in Figure 4.3. The blade geometry was modified in this way to avoid extreme velocities and pressure gradients at the trailing edge. The inviscid flow solution in the region of the trailing edge could then be used directly by the boundary layer computations. This approximation represents a small compromise in the accuracy of the computational predictions for repeatability of the predictions. Section 4.3.3 describes some of the implications of modifying the trailing edge geometry.

In order to modify the trailing edge of the actual blade, curves were fit to three of the original blade coordinates on both the upper and lower surfaces. The first of the three coordinates chosen was at approximately $85 \%$ axial chord downstream of the leading edge and the third coordinate was taken as the centre point on the trailing edge (see Figure 4.3). Several additional surface coordinates were then selected on the curves on both surfaces. These became junction points in the panel-method computations. The modified blade profile was tested by visually inspecting the pressure distribution determined by the inviscid computations. By "trial and error" small alterations of the newly obtained coordinates were sometimes necessary to develop a smooth flow over the modified region. Appendix $G$ contains a sample input data file with the coordinates of the modified biade profile. 
51

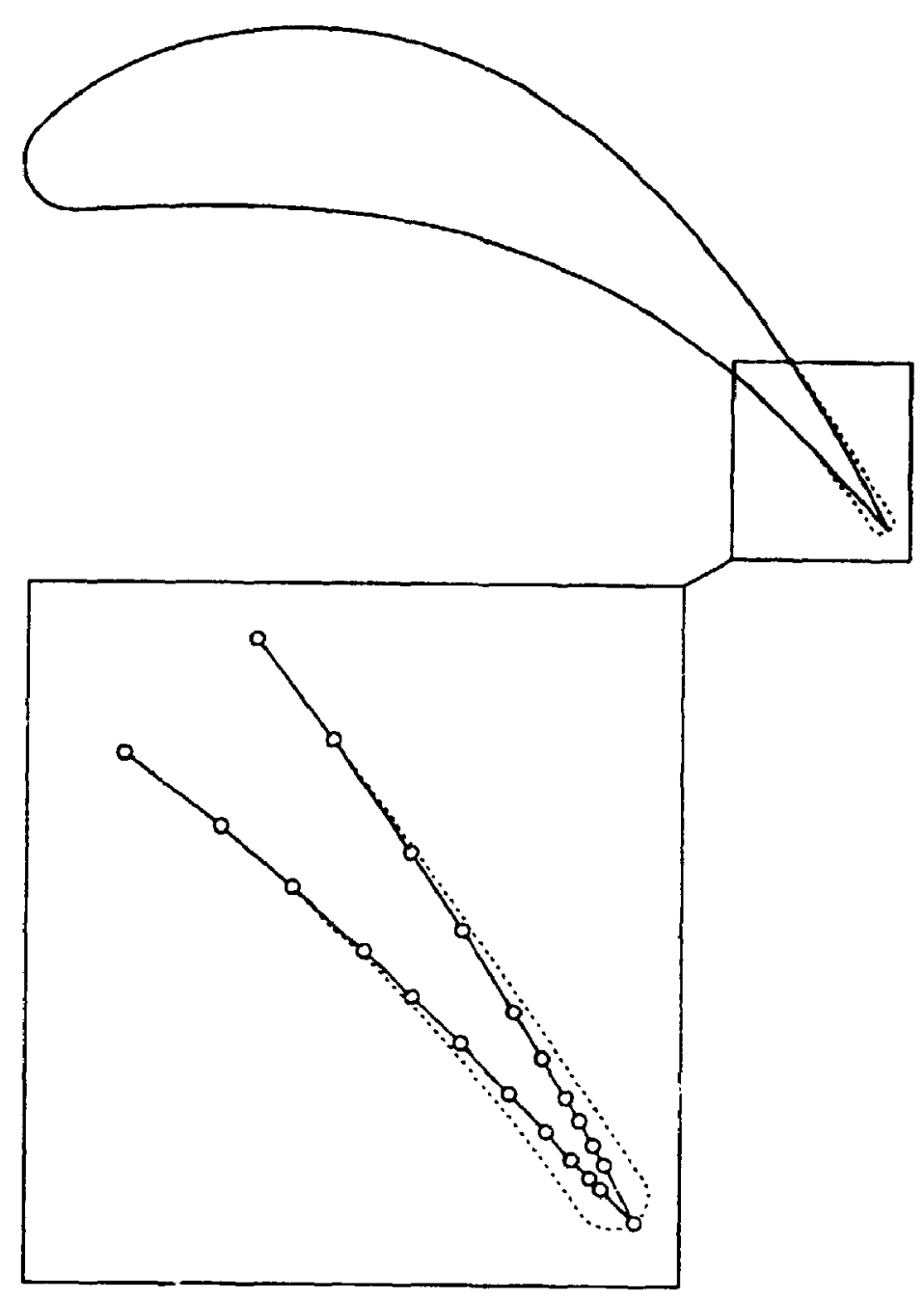

Original Blade

Modified Blade

Figure 4.3: Original and modified blade profile. 


\subsubsection{Kutta Condition}

A Kutta condition was applied to give a smooth flow over the trailing edge of the cascade tiades. Various forms of the Kutta condition exist and three versions were available in the panel-method program, namely:

(1) Vorticity magnitude (absolute values) on upper and lower surface at the trailing edge set equal to one another, the value being unconstrained.

(2) Trailing edge made a stagnation point.

(3) Vorticity magnitude (absolute values) on upper and lower surface at the trailing edge set equal to one another with the value constrained to equal the mean of the nearby upstream values on the upper and lower surface.

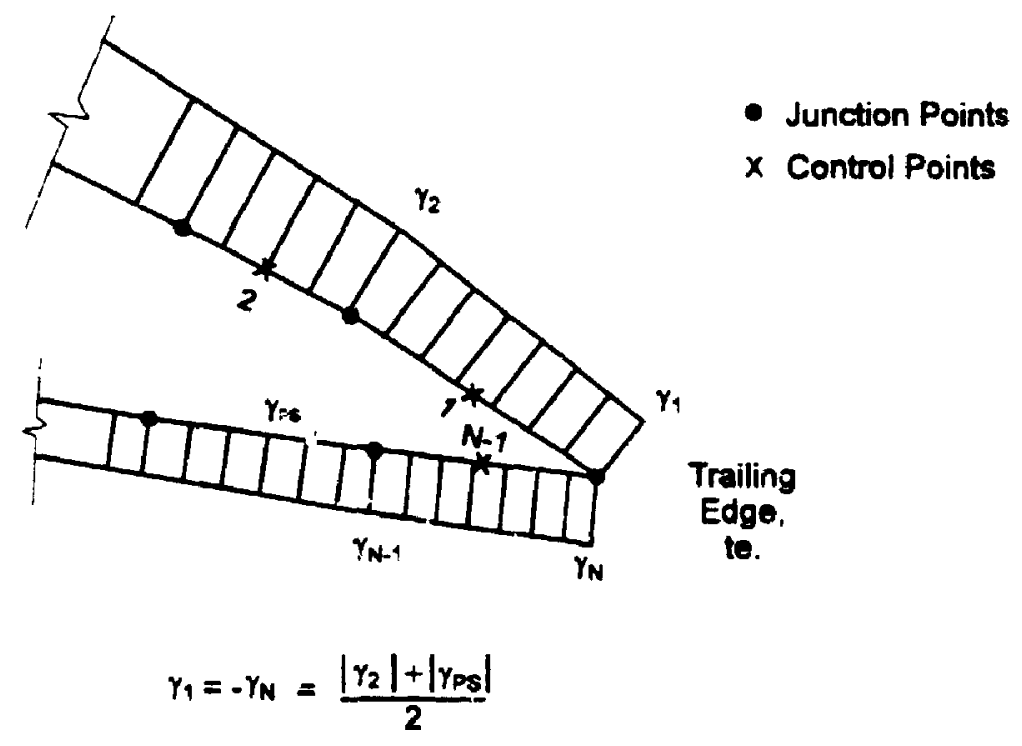

Figure 4.4: Schematic illustration of the vorticity distribution at the trailing edge for the third form of the Kutta condition. 
Kutta conoitions (1) and (3) are based on the requirement that zero net vorticity must be shed inte the wake in steady flow, in accordance with Kelvin's theorem. Figure 4.4 shows a schematic illustration of the vorticity distribution at the trailing edge for the third form of the Kutta condition. Kutta condition (3) gave somewhat better behaviour than the other two near the trailing edge and hence gave better source distributions by the boundary layer analysis code.

\subsubsection{Computational Procedures Practices}

\subsubsection{Cascade Configuration}

Figure 4.5 shows a cascade and defines the cascade nomenclature used with the computational model. The axial chord of the primary profile was chosen as the $x$ axis of the global $x, y$ coordinate system. The inflow and outflow angles, $\alpha_{1}$ and $\alpha_{2}$, are measured relative to the $x$ axis and are considered positive when there is a positive velocity component in the plus $y$ direction. The stagger angle, $y$, is normally defined as the angle of the chordline with respect to the throughflow direction for the cascade, as shown in Figure 4.5; however, in the present computations the axial chord, $c_{x}$, was chosen as the $x$ axis instead of the chordline so the stagger angle for input to the computer program is considered zero. The blade coordinate values and the spacing between each blade are normalized by the axial chord. Thirty companion blades were used to approximate an infinite cascade and ninety vortex panels were used to represent the blade surface.

\subsubsection{Boundary Layer Transition}

Figure 4.6 shows a typical pressure distribution obtained experimentally and one predicted by the inviscid flow computations for the same conditions. Both the 

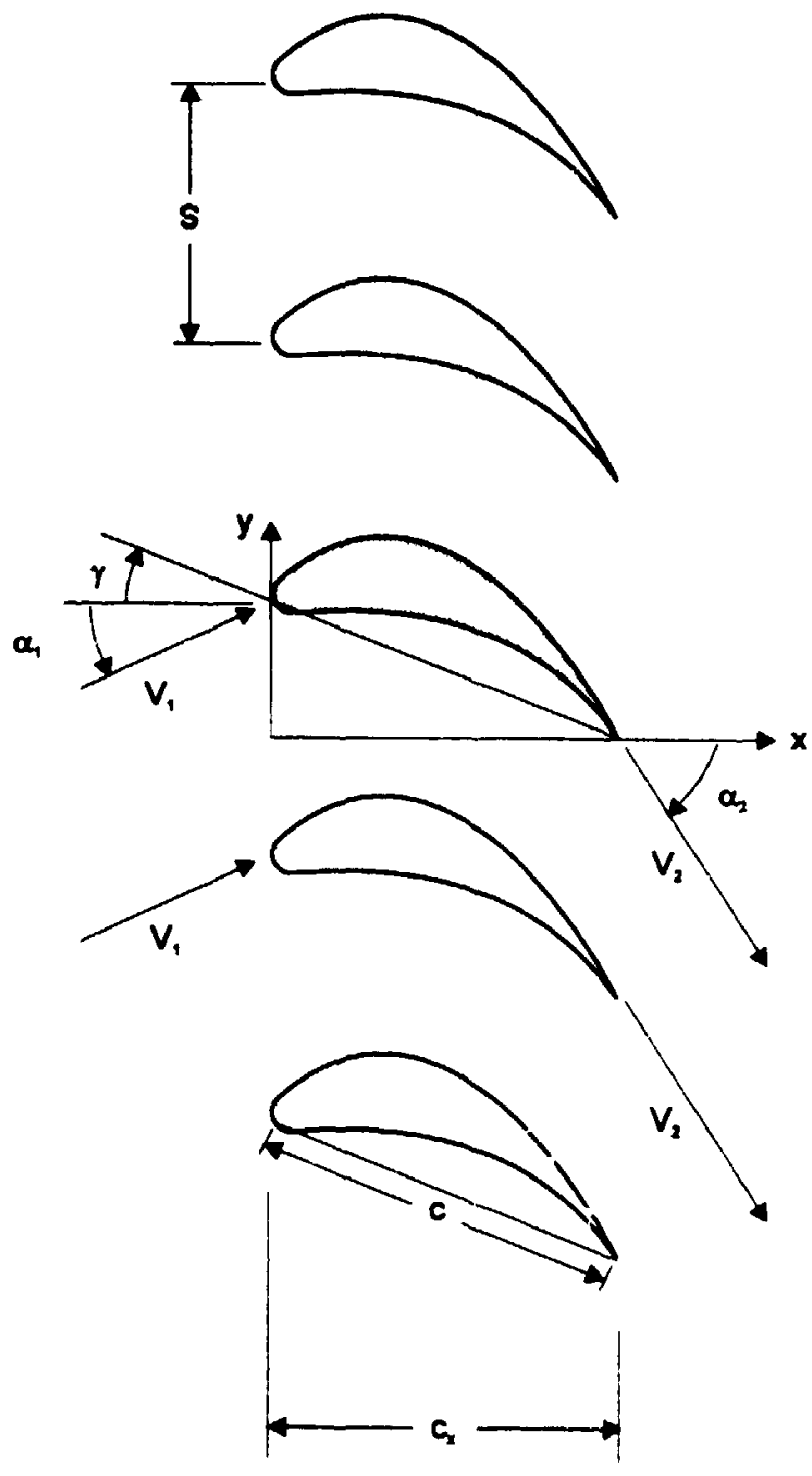

Primary Profice

Stayger Angie, $\gamma$ Inlet Flow Angle

$0^{\circ}$

Estimate of Outlet Flow Angle

: $\alpha_{1} \quad$ (e.g. $\alpha_{1}=28.35^{\circ}$ at design incidence)

Blade Spacing, S/C

$: a_{2}$

Number of Companion Blades, NBLS

0.741

(e.g. $\alpha_{2}=-55.00^{\circ}$ at design incidence)

30

Figure 4.5: Input parameters for computations on the present cascade. 


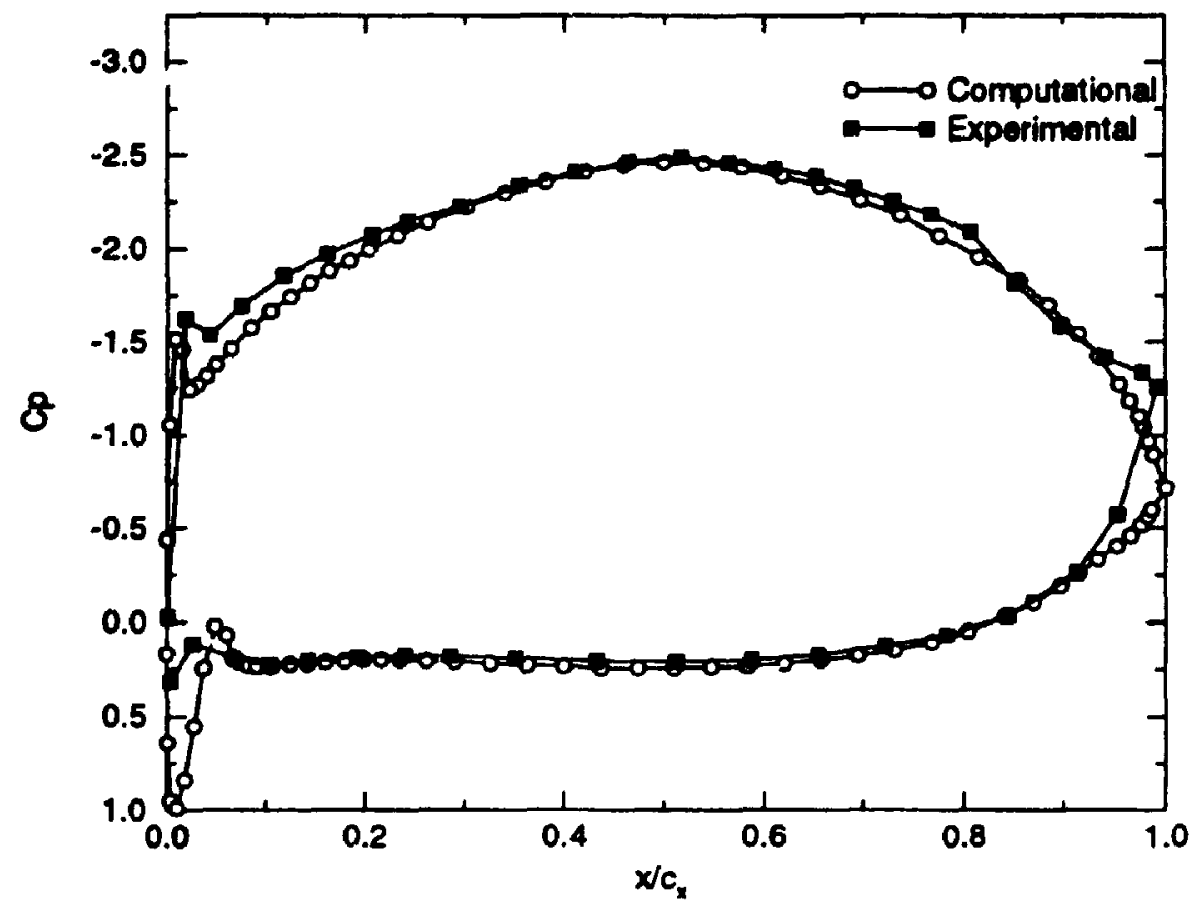

Figure 4.6: Typical pressure distribution determined both experimentally and computationally.

experimental data and the computational prediction show evidence of a pressure-surface and suction-surface leading edge velocity spike or 'overspeed'. Benner et al. (1995) examining the same blade profile attributes the leading edge velocity spikes to a change in surface curvature there. As can be seen, the overspeeds predicted by the potential flow code tends to be somewhat larger than those in the experimental data. The strong adverse pressure gradient immediately after the overspeed can result in the incorrect prediction of laminar to turbulent transition by the $H-R_{x}$ method or of laminar flow separation by Thwaites' method in the boundary layer analysis code. Therefore, one must rely on experimental evidence to determine whether the boundary layer is laminar or turbulent, separated or attached. For example, for smooth blades at design incidence, experimental evidence by Whitehouse (1993) and this author (discussed in Chapter 5) suggests that laminar flow exists on the suction-surface from the leading edge up to approximately mid chord. Also, experimental data acquired by this author suggests that laminar flow exists over most of the pressure-surface. Based on these findings, if the boundary layer analysis 
predicted transition or flow separation near the leading edge, the local velocity values from the potential flow solution at the velocity spike were adjusted slightly such that the $H-R_{x}$ or Thwaites' methods would not erroneously predict transition or separation. This procedure was used for all cascade incidence angles that were investigated.

The boundary-layer momentum thickness, $\theta$, was assumed to remain constant through transition on smooth upper and lower surfaces. In the case of transition on rough surfaces, a momentum thickness value based on the roughness height, $k$ and spacing parameter, $\lambda$, was assumed at the start of the turbulent calculations (i.e. $\theta=10 \mathrm{k} / \lambda$ ). The initial turbulent shape factor $H$, was assigned the value of 1.4 at the start of all turbulent calculations. Instantaneous transition was assumed.

\subsubsection{Surface-Source Distribution}

Computations were done for various extents of surface roughness on the upper and lower surfaces of the profile. The non-dimensional roughness height, $k / c_{x}$, was made to increase approximately linearly over a short distance (approximately $0.1 \Delta s / c_{x}$ ) at the upstream edge of roughness bands. This is both more realistic than an abrupt start and also avoids potential difficulties with the boundary layer computations that an abrupt start with large $k / c_{x}$ might have caused. A boundary layer step size of $\Delta s / c_{x}=0.01$ was used for all cases investigated. Only one iteration was used between the inviscid and boundary-layer computations (i.e. inviscid-viscous-inviscid).

The surface-source distribution determined by the boundary layer computations would sometimes entail the modification and/or "smoothing" of some of the source strengths in the trailing edge region prior to performing a second inviscid pass. The boundary layer computations on the suction surface in some cases investigated (i.e. large surface roughness heights and roughness band widths or large incidence angles) would 
predict separation near the trailing edge. For these cases the upper surface separation point was predicted anywhere between $95 \%$ to $100 \%$ of axial chord downstream of the leading edge. The boundary layer analysis program deals with separated flow by setting the source-strength values to zero on the panels downstream of the separation point. Because the separation bubble could be considered small (i.e. less than $5 \%$ of axial chord) its effects were crudely approximated by adding source strengths manually in the separation region prior to performing the second inviscid pass. Also, because of extremely rapid predicted increases of the boundary layer shape factor, $H$, the surfacesource values immediately upstream of the separation region would sometimes be unrealistic, requiring some manual "smoothing" prior to a second inviscid pass. The computed pressure distributions and losses were relatively insensitive to variations in the source strengths in the trailing-edge region, provided that there were no sharp discontinuities or extreme values. Thus the modifications mentioned here served only to avoid numerical difficulties with the computational model and did not introduce significant arbitrariness into the results.

\subsection{Post-Processing of Computational Results}

\subsubsection{Pressure Coefincients}

The pressure coefficients obtained experimentally are based on cascade inlet conditions as discussed in Section 3.3.6, whereas the pressure coefficients predicted by the computational model are based on the mean velocity through the cascade. In order to compare the two pressure coefficient values (experimental and computational) the following conversion is performed on the computational predictions:

$$
C p_{\text {comp }}=1-\left(1-C p_{\text {comp }}^{\prime}\right) \frac{\bar{V}^{2}}{V_{1}^{2}}
$$


where $C_{p_{\text {comp }}^{\prime}}^{\prime}$ denotes the pressure coefficient predicted computationally, $V_{1}{ }^{2}$ is the cascade inlet velocity squared and $\tilde{V}^{2}$ is the mean velocity squared through the cascade (i.e. $\left.\bar{V}^{2}=\left(V_{1}^{2}+V_{2}^{2}\right)\right)$. The resulting pressure coefficient, $C P_{\text {comp }}$, is based on the inlet conditions of the cascade.

\subsubsection{Loss Prediction Schemes}

The profile losses are taken to include the loss production in the blade-surface boundary layers as well as trailing edge blade-drag effects. The loss coefficient (derived in detail in Appendix $\mathrm{H}$ ) takes the form:

$$
\frac{\Delta P_{o}}{\frac{1}{2} \rho V_{2}^{2}}=\frac{2 \theta_{\text {wake }}}{S \cos \alpha_{2}}+Y_{\text {tet }}
$$

where $S$ is blade to blade spacing and $\alpha_{2}$ is the outlet flow angle. The first term accounts for the direct loss production in the boundary layers and the second term, $Y_{\text {tee }}$, is the trailing edge base-loss contribution. The wake momentum thickness far downstream, $\theta_{\text {wake }}$ must be related to the momentum thickness calculated at the trailing edge by the boundary-layer computations. The semi-empirical relation of Squire and Young (1938) is used for this purpose:

$$
\boldsymbol{\theta}_{\text {wake }}=\left(\theta_{u p}+\theta_{\text {low }}\right)\left(\frac{\bar{U}_{\text {te }}}{U_{\text {far }}}\right)^{3.2}
$$

where $\theta_{u p}$ and $\theta_{\text {low }}$ are the trailing edge momentum thickness values predicted by the boundary-layer computations on the upper and lower blade surfaces respectively, $\bar{U}_{\text {re }}$ is the mean velocity at the trailing edge upper and lower surfaces calculated by the inviscid 
solution during the second pass and $U_{\text {far }}$ is the velocity far downstream of the trailing edge defined as $U_{\text {for }}=V_{2}=V_{1} \cos \alpha_{1} / \cos \alpha_{2}$ by continuity.

The profile loss coefficient is determined solely by $\theta_{\text {mate }}$ if the trailing edge is sharp. A trailing edge thickness contribution must be added in the case of blunt trailing edges. The correlation of Kacker and Okapuu (1982) is used for this purpose. They defined the trailing edge losses, $Y_{\text {ur }}$ in terms of the trailing edge thickness/throat opening ratio (i.e. $t / \sigma$ )of a cascade. Two distinct curves exist for axial entry nozzles and impulse blades. Figure 4.7 shows the two curves and the interpolating equation used to deduce the trailing edge loss (energy) coefficient.

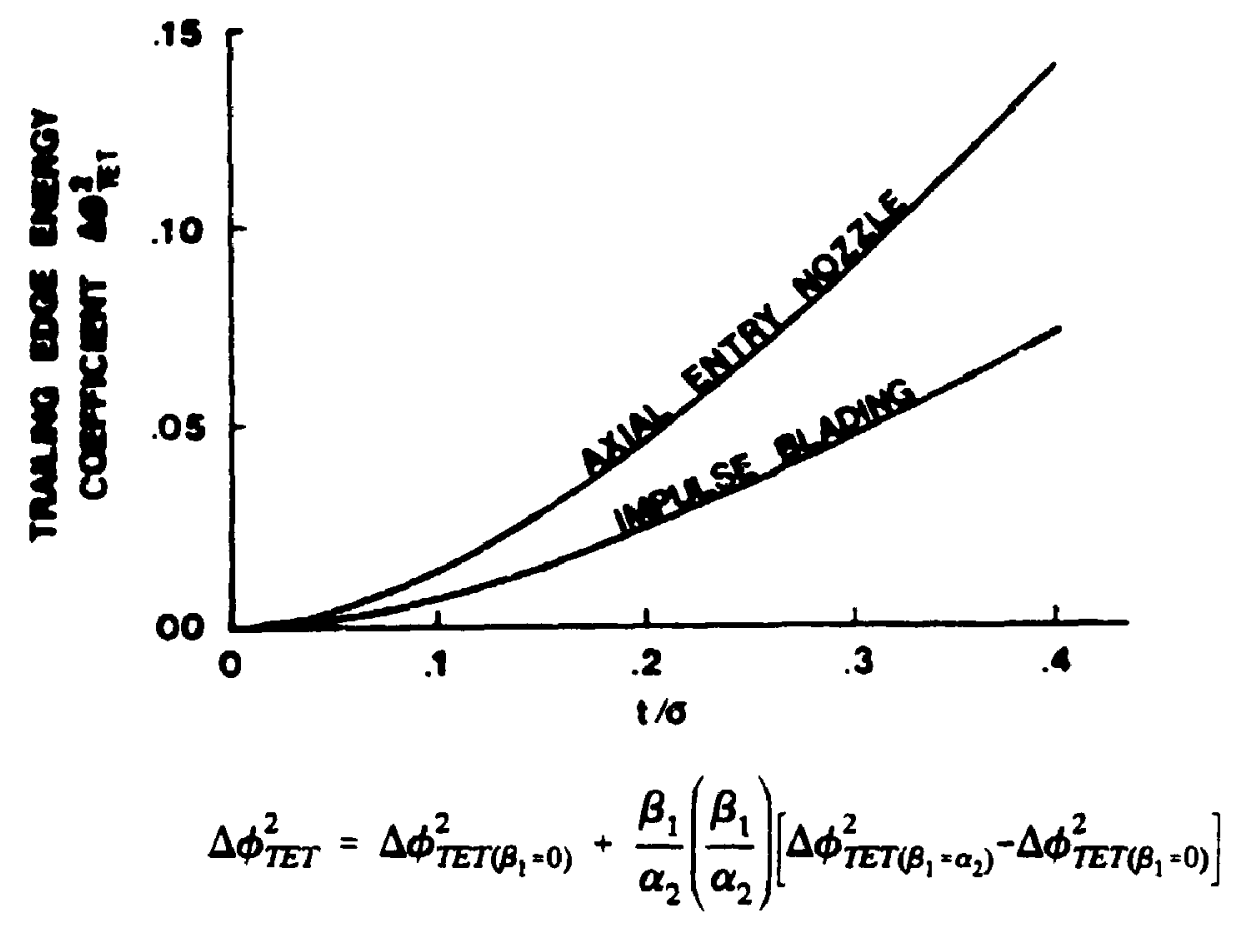

Figure 4.7: Trailing edge loss (energy) coefficient correlated against the ratio of twaling edge thickness to throat opening (where $\beta_{1}$-inlet blade metal angle, $\alpha_{2}$-outlet flow angle) - reproduced from Kacker and Okapuu (1982)). 
The conversion from the kinetic energy loss coeffic:ent to a total-pressure loss coefficient is given by

$$
Y_{\text {tet }}=\frac{1}{1-\Delta \phi_{\text {tet }}^{2}}-1
$$

for incompressible flow (Tremblay (1989).

\subsection{Potential Sources of Error}

It would be very difficult to accurately quantify the error in predicting the various aerodynamic parameters due to the many variables involved. The following section describes some of the key areas where error could occur.

Section 4.2.1 describes the modifications made to the trailing edge of the actual blade profile. Modifications to the trailing edge region can lead to inaccuracies in the predictions of the outlet flow angle, base pressure, and boundary-layer development. In order to verify the implications of using the modified blade geor..ztry the following work was performed. Source strengths were added to the trailing edge region prior to performing a second inviscid pass. The values of the additional source strengths were calculated based on the local inviscid velocities and the 'displacements' between the modified geometry (see Figure 4.8). This approach is based on the same principle as the use of sources to represent boundary-layer displacement thickness effects. Once the source strengths were added the second inviscid pass was performed followed by a second viscous pass. The resulting outlet flow angle, base pressure, and pressure loss coefficient were compared for the corrected and non-corrected computations. The differences in outflow angles and base pressures were found to be insignificant (less than $0.01 \%$ ) and the difference in the pressure loss coefficient was less than $1 \%$. Since the differences between the two results were small, no corrections for the modified trailing edge 
geometry were applied for the results presented in Chapter 5.

The semi-empirical reiation due to Squire and Young (1938), described in Section 4.3.2, can result in prediction errors for total-pressure loss coefficients. Squire and Young (1938) based their relation on the integral-momentum equation together with data from airfoils at small angles of attack. Since there is no corresponding data for cascades, and since one can expect a cascade blade wake to develop in essentially the same way as the wake of an isolated airfoil the Squire and Young relation is used. At large cascade off-design incidence angies the result due to Squire and Young was also used despite the fact that their empirical relation was based on small angles of attack.

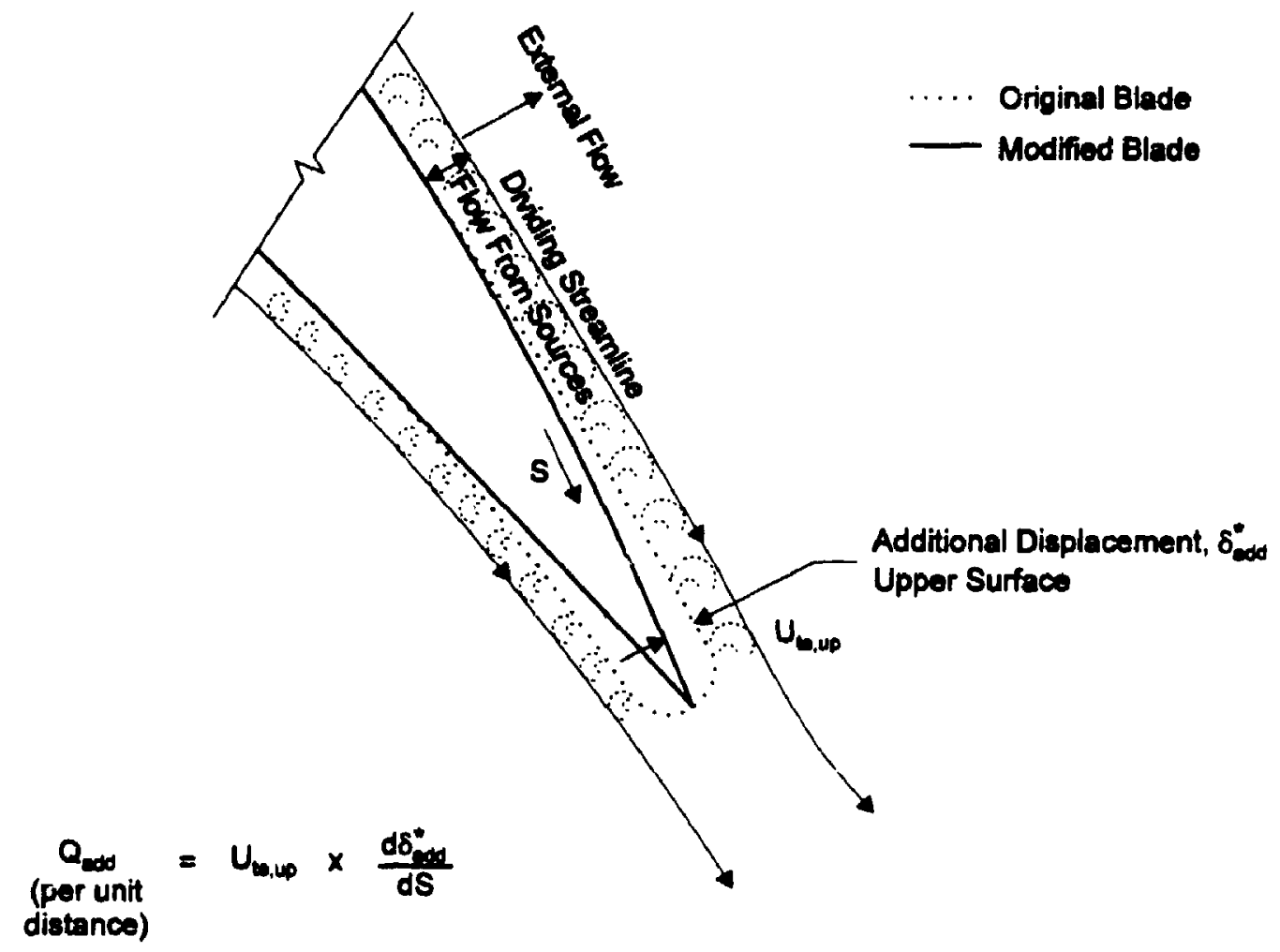

Figure 4.8: Schematic representation of the trailing edge with the additional sourc strength scheme. 


\section{Chapter 5}

EXPERIMENTAL AND COMPUTATIONAL RESULTS

This chapter presents both the computational and experimental results. The experimental results are presented in Section 5.1 and the computational results are presented and compared to the experimental results in Section 5.2 .

\subsection{Experimental Results}

The two main parameters which will be used to analyze the flow are the massaveraged total-pressure loss, $Y_{m}$, and the deviation angle, $\delta$. There exists a close relationship between the blade pressure distributions and the development of the boundary layer. Therefore, prior to presenting the total-pressure loss and deviation angle data, midspan static pressure measurements will be presented. Table 5.1 and $5.1 \mathrm{~b}$ give a summary of all test cases investigated along with the total-pressure loss and flow deviation data by Abbott (1993) and from the present study. The curves presented in the figures to follow will be identified by the Trial \# listed in Table 5.1. The experimental data determined during the present study are presented along with the axial velocity ratios (AVR) and the approximate point at which the laminar boundary layer begins transition to a turbulent boundary layer. Also shown in Table 5.1 are the computational predictions: these will be discussed in detail in Section 5.2 of this Chapter. Figure 5.1 illustrates the locations and the roughness band widths of the various samples on the blade profile. 
Table 5.1: Rougíness configurations, measured and predicted data (experimental data reproduced from Abbott (1993)).

\begin{tabular}{|c|c|c|c|c|c|c|c|c|c|}
\hline \multicolumn{6}{|c|}{ Roughness Configurauons } & \multicolumn{2}{|c|}{ Experimental } & \multicolumn{2}{|c|}{ Compurational } \\
\hline Trial & $\begin{array}{c}\text { Roughoess } \\
\text { LE } \\
\text { Position } \\
\text { SS*, PS }^{* *} \\
; / c\end{array}$ & $\begin{array}{c}\text { Sand } \\
\text { Grain } \\
\text { Size } \\
\text { W/c }\end{array}$ & $\begin{array}{l}\text { : Jhoss } \\
\text { Baud } \\
\text { Widtb } \\
\Delta s / c\end{array}$ & $\begin{array}{l}\text { Incideose } \\
\text { Angle }\end{array}$ & $\begin{array}{l}\text { Spacing } \\
\text { Parameter }\end{array}$ & $\begin{array}{c}\text { Totai } \\
\text { Pressure } \\
\text { Loss } \\
Y_{\mathrm{a}}\end{array}$ & $\begin{array}{c}\text { Fow } \\
\text { Devia- } \\
\text { tion }\end{array}$ & $\begin{array}{c}\text { Total } \\
\text { Pressure } \\
\text { Loss } \\
\mathbf{Y}_{\text {. }}\end{array}$ & $\begin{array}{c}\text { Flow } \\
\text { Devia- } \\
\text { vion } \\
\delta\end{array}$ \\
\hline $12 \mathrm{a}$ & $\cdots$ & $\cdots$ & $\cdots$ & $\sigma^{\circ}$ & - & $0.6: 75$ & $-3.99^{\circ}$ & 0.0235 & $-4.66^{\circ}$ \\
\hline $12 b$ & $\cdots$ & $\cdots$ & $-\cdots$ & $+10^{\circ}$ & - & 0.0327 & $-3.41^{\circ}$ & 0.0329 & $.5 .14^{\circ}$ \\
\hline $12 \mathrm{c}$ & $\cdots$ & $\cdots$ & $\cdots$ & $-10^{\circ}$ & .. & 0.0279 & $-4.15^{\circ}$ & 0.0241 & $-4.65^{\circ}$ \\
\hline $1 d$ & -- & -- & $\cdots$ & $+20^{\circ}$ & -. & 0.2370 & $-9.82^{\circ}$ & $\ldots$ & - \\
\hline $1 e$ & $\cdots$ & $\cdots$ & - & $-20^{\circ}$ &.. & $0.05 v 4$ & $-3.94^{\circ}$ & 0.0239 & $-4.66^{\circ}$ \\
\hline $2 \mathrm{a}$ & 0.645 SS & 0.00209 & 0.235 & $0^{\circ}$ & 7.4 & 0.0316 & $-4.29^{\circ}$ & 0.0314 & $-5.23^{\circ}$ \\
\hline $2 b$ & $0.645 \mathrm{SS}$ & 0.00208 & 0.235 & $+10^{\circ}$ & 7.4 & 0.0372 & $-4.32^{\circ}$ & 0.0397 & $-5.33^{\circ}$ \\
\hline $2 c$ & 0.645 SS & 0.00208 & 0.235 & $-10^{\circ}$ & 7.4 & 0.0302 & $-4.35^{\circ}$ & 0.0320 & $-5.48^{\circ}$ \\
\hline $2 d$ & 0.645 SS & 0.00208 & 0.235 & $+20^{\circ}$ & 7.4 & 0.1763 & $-9.24^{\circ}$ & $\cdots$ & - \\
\hline $2 e$ & $0.645 \mathrm{SS}$ & 0.00208 & 0.235 & $-20^{\circ}$ & 7.4 & 0.0319 & $-4.61^{\circ}$ & 0.0314 & $-5.76^{\circ}$ \\
\hline $3 a$ & 0.645 SS & 0.00445 & 0.235 & $\sigma^{\infty}$ & 10.0 & 0.0310 & $-4.47^{\circ}$ & 0.0338 & $-5.39^{\circ}$ \\
\hline $3 b$ & $0.645 \mathrm{SS}$ & 0.00445 & 0.235 & $+10^{\circ}$ & 10.0 & 0.0358 & $-3.86^{\circ}$ & 0.0407 & $-5.60^{\circ}$ \\
\hline $3 c$ & 0.645 SS & 0.00445 & 0.235 & $-10^{\circ}$ & 10.0 & 0.0317 & $-4.05^{\circ}$ & 0.0348 & $-5.67^{\circ}$ \\
\hline $4 a$ & 0.534 SS & 0.00445 & 0.470 & $+0^{\circ}$ & 9.0 & 0.0367 & $-4.38^{\circ}$ & $\cdots$ & -. \\
\hline $4 b$ & 0.534 SS & 0.00445 & 0.470 & $+10^{\circ}$ & 9.0 & 0.0363 & $-3.82^{\circ}$ & -- & -. \\
\hline $5 a$ & $0.250 \mathrm{SS}$ & 0.00445 & 0.470 & $\sigma^{\circ}$ & 13.7 & 0.0543 & $-478^{\circ}$ & 0.0510 & $-6.11^{\circ}$ \\
\hline $5 b$ & $0.250 \mathrm{SS}$ & 0.00445 & 0.470 & $+10^{\circ}$ & 13.7 & 0.0573 & $-4.51^{\circ}$ & 0.0543 & $.5 .79^{\circ}$ \\
\hline $5 \mathrm{c}$ & $0.250 \mathrm{SS}$ & 0.00445 & 0.470 & $-10^{\circ}$ & 13.7 & 0.0572 & $-5.76^{\circ}$ & 0.0507 & $-6.33^{\circ}$ \\
\hline Sd & $0.250 \mathrm{SS}$ & 0.00445 & 0.470 & $+20^{\circ}$ & 13.7 & 0.2247 & $-9.56^{\circ}$ & $\ldots$ & $\because$ \\
\hline $5 \mathrm{e}$ & $0.250 \mathrm{SS}$ & i 20445 & 0.470 & $.20^{\circ}$ & 13.7 & 0.0567 & $-4.64^{\circ}$ & 0.0502 & $-5.99^{\circ}$ \\
\hline
\end{tabular}


Table 5.1: (cont.) Roughness configurations, measured and predicted data (experimental data reproduced from Abbott (1993)).

\begin{tabular}{|c|c|c|c|c|c|c|c|c|c|}
\hline \multicolumn{6}{|c|}{ Roughness Configurations } & \multicolumn{2}{|c|}{ Experimental } & \multicolumn{2}{|c|}{ Compulational } \\
\hline T\# & $\boldsymbol{x} / \mathbf{c}$ & $\mathbf{k} / \mathbf{c}$ & $\Delta e / c$ & $i$ & $\lambda$ & $\mathbf{Y}_{\mathbf{n}}$ & $\boldsymbol{\delta}$ & $\mathbf{Y}_{\mathbf{m}}$ & $\boldsymbol{\delta}$ \\
\hline $6 a$ & $0.250 \mathrm{SS}$ & 0.00445 & 0.235 & $\infty^{\circ}$ & 13.7 & 0.0477 & $-4.93^{\circ}$ & 0.0435 & $-5.68^{\circ}$ \\
\hline $6 \mathrm{~b}$ & $0.250 \mathrm{SS}$ & 0.00445 & 0.235 & $+10^{\circ}$ & 13.7 & 0.0491 & $.4 .47^{\circ}$ & 0.0471 & $-5.70^{\circ}$ \\
\hline $6 \mathrm{c}$ & $0.250 \mathrm{SS}$ & 0.00445 & 0.235 & $-10^{\circ}$ & 13.7 & 0.0468 & $-5.47^{\circ}$ & 0.0406 & $-5.71^{\circ}$ \\
\hline $7 \mathbf{a}$ & $0.250 \mathrm{SS}$ & 0.00208 & 0.470 & $\sigma^{\circ}$ & 8.8 & 0.0464 & $-4.81^{\circ}$ & 0.0501 & $-6.08^{\circ}$ \\
\hline $7 \mathrm{~b}$ & $0.250 \mathrm{SS}$ & 0.00208 & 0.470 & $+10^{\circ}$ & 8.8 & 0.0494 & $-4.46^{\circ}$ & 0.0501 & $-5.70^{\circ}$ \\
\hline $7 \mathrm{c}$ & $0.250 \mathrm{SS}$ & 0.00208 & 0.470 & $-10^{\circ}$ & 8.8 & 0.0481 & $-5.19^{\circ}$ & 0.0504 & $-6.17^{\circ}$ \\
\hline $8 \mathrm{a}$ & $0.250 \mathrm{SS}$ & 0.00208 & 0.235 & $\sigma$ & 8.8 & 0.0405 & $-4.35^{\circ}$ & $0.045 r$ & $-5.80^{\circ}$ \\
\hline $8 b$ & $0.250 \mathrm{SS}$ & 0.00208 & 0235 & $+10^{\circ}$ & 8.8 & 0.0438 & $-4.08^{\circ}$ & 0.0448 & $-5.59^{\circ}$ \\
\hline $8 c$ & $0.250 \mathrm{SS}$ & 0.00208 & 0.235 & $-10^{\circ}$ & 8.8 & 0.0413 & $-4.45^{\circ}$ & 0.0437 & $-5.60^{\circ}$ \\
\hline $9 \mathbf{a}$ & $0.250 \mathrm{SS}$ & 0.00628 & 0.470 & $\sigma^{\circ}$ & 15.9 & 0.0605 & $-4.01^{\circ}$ & 0.0533 & $-5.80^{\circ}$ \\
\hline $10 \mathrm{a}$ & $0.250 \mathrm{SS}$ & 0.00628 & 0235 & $\sigma^{\circ}$ & 15.9 & 0.0551 & $-4.72^{\circ}$ & 0.0446 & $-5.83^{\circ}$ \\
\hline $11 \mathrm{a}$ & $0.250 \mathrm{sS}$ & 0.00208 & 0.470 & $\sigma^{\circ}$ & 10.0 & .0458 & $-4.50^{\circ}$ & 00482 & $-5.91^{\circ}$ \\
\hline
\end{tabular}


Table 5.1b: Roughness configurations, measured and predicted data (all data shown are from the present study).

\begin{tabular}{|c|c|c|c|c|c|c|c|c|c|c|c|}
\hline \multicolumn{6}{|c|}{ Roughness Config Irations } & \multicolumn{4}{|c|}{ Experimental } & \multicolumn{2}{|c|}{ Computational } \\
\hline T* & $\boldsymbol{x} / \mathbf{c}$ & $\mathbf{b} / \mathbf{c}$ & Ank & $i$ & $\lambda$ & $\mathbf{Y}_{\mathbf{0}}$ & 8 & $\mathbf{A} \mathbf{V R}$ & $\boldsymbol{v} / \mathrm{c}$ & $\mathbf{Y}_{\mathbf{D}}$ & $\boldsymbol{\delta}$ \\
\hline 1 & --- & $\cdots$ & -- & $-10^{\circ}$ & $\because$ & 0.0282 & $-4.6^{\circ}$ & 0.997 & $0.70 \mathrm{SS}$ & 0.0241 & $-4.65^{\circ}$ \\
\hline $1 \mathrm{~b}$ & -- & -- & $\cdots$ & $0^{\circ}$ & -- & 0.0280 & $-4.2^{\circ}$ & 0.995 & $0.28 \mathrm{SS}$ & 0.0235 & $-4.66^{\circ}$ \\
\hline 18 & $\cdots$ & -- & $\cdots$ & $+10^{\circ}$ & 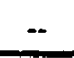 & 0.0388 & $-4.9^{\circ}$ & 1.005 & - & 0.0329 & $-5.14^{\circ}$ \\
\hline $1 \mathrm{c}$ & - & -- & $\cdots$ & $+15^{\circ}$ & -- & 0.0948 & $-8.1^{\circ}$ & 1.006 & - & -.. & -- \\
\hline $13 \mathrm{a}$ & $0.25 \mathrm{SS}$ & 0.00208 & 0.47 & $0^{\circ}$ & 11.1 & 0.0459 & $-4.4^{\circ}$ & 1.010 & - & 0.0456 & $-5.31^{\circ}$ \\
\hline 9 a & $0.25 \mathrm{SS}$ & 0.00628 & 0.47 & $\infty^{\circ}$ & 10.2 & 0.0654 & $-4.7^{\circ}$ & 1.002 & $-\cdot$ & 0.0626 & $-5.76^{\circ}$ \\
\hline $9 \mathrm{~b}$ & $0.25 \mathrm{SS}$ & 0.00628 & 0.47 & $+10^{\circ}$ & 10.2 & 0.0761 & $-4.9^{\circ}$ & 1.000 & -- & 0.0604 & $-6.43^{\circ}$ \\
\hline $9 c$ & $0.25 \mathrm{SS}$ & 0.00628 & 0.47 & $+15^{\circ}$ & 10.2 & 0.1849 & $-8.9^{\circ}$ & 1.007 & - & $\cdots$ & -- \\
\hline $10 a$ & $0.25 \mathrm{SS}$ & 0.00628 & 0.24 & $0^{\circ}$ & 10.2 & 0.0544 & $-4.8^{\circ}$ & 0.999 & $0.26 \mathrm{SS}$ & 0.0505 & $-5.38^{\circ}$ \\
\hline $10 \mathrm{~b}$ & 0.25 SS & 0.00628 & 0.24 & $+10^{\circ}$ & 10.2 & 0.0634 & -517 & 1.000 & 0.04 SS & 0.0500 & $-6.13^{\circ}$ \\
\hline $10 \mathrm{c}$ & $0.25 \mathrm{SS}$ & 0.00628 & 0.24 & $+15^{\circ}$ & 10.2 & 0.1642 & $-9.1^{\circ}$ & 1.007 & 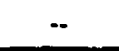 & $\cdots$ & $\because$ \\
\hline $14 a$ & $0.10 \mathrm{SS}$ & 0.000445 & 0.47 & $0^{\circ}$ & 11.0 & 0.0648 & $-4.8^{\circ}$ & 0.996 & $0.13 \mathrm{sS}$ & 0.0505 & $.6 .15^{\circ}$ \\
\hline $14 \mathrm{~b}$ & $0.10 \mathrm{SS}$ & 0.00445 & 0.47 & $+10^{\circ}$ & 11.0 & 0.0731 & $-4.9^{\circ}$ & 0.993 & 0.03 SS & 0.0510 & $-5.86^{\circ}$ \\
\hline $14 \mathrm{c}$ & $0.10 \mathrm{SS}$ & 0.00445 & 0.47 & $+15^{\circ}$ & 11.0 & 0.2042 & $-9.2^{\circ}$ & 1.000 & $-\cdot$ & -- & -. \\
\hline 152 & $0.10 \mathrm{SS}$ & 0.00445 & 0.24 & $0^{\circ}$ & 11.0 & 0.0507 & $-4.9^{\circ}$ & 1.001 & 0.14 SS & $0.041 ?$ & $-5.85^{\circ}$ \\
\hline $15 \mathrm{~b}$ & $0.10 \mathrm{SS}$ & 0.00445 & 0.24 & $+10^{\circ}$ & 11.0 & 0.0620 & $-5.1^{\circ}$ & 0.993 & $\cdots$ & 0.0474 & $-5.92^{\circ}$ \\
\hline $15 \mathrm{c}$ & $0.10 \mathrm{SS}$ & 0.00445 & 0.24 & $+15^{\circ}$ & 11.0 & 0.1419 & $-8.7^{\circ}$ & 1.003 & $0.02 \mathrm{SS}$ & $--\cdot$ & .. \\
\hline
\end{tabular}


Table 5.1b: (cont.) Roughness configurations and measured and predicted data (all data shown are from the present study).

\begin{tabular}{|c|c|c|c|c|c|c|c|c|c|c|c|}
\hline \multicolumn{6}{|c|}{ Roughness Configurations } & \multicolumn{4}{|c|}{ Experimental } & \multicolumn{2}{|c|}{ Computational } \\
\hline T* & $\boldsymbol{x} / \mathrm{c}$ & $\mathbf{k} / \mathbf{c}$ & Aete & $\boldsymbol{i}$ & $\lambda$ & $\mathbf{Y}_{\mathbf{E}}$ & $\boldsymbol{\delta}$ & $\mathbf{A V R}$ & $\boldsymbol{x} / \mathbf{c}$ & $\mathbf{Y}_{\mathbf{m}}$ & $\boldsymbol{\delta}$ \\
\hline $16 \mathrm{a}$ & $0.25 \mathrm{PS}$ & 0.00445 & 0.71 & $-10^{\circ}$ & IC.0 & 0.0332 & $-4.9^{\circ}$ & 0.997 & $0.51 \mathrm{SS}$ & 0.0293 & $-4.71^{\circ}$ \\
\hline $16 \mathrm{~b}$ & 0.25 PS & 0.00445 & 0.71 & $0^{\circ}$ & 10.0 & 0.0360 & $-4.2^{\circ}$ & 0.993 & $0.31 \mathrm{PS}$ & 0.0307 & $-4.74^{\circ}$ \\
\hline $16 \mathrm{c}$ & 0.25 PS & 0.00445 & 0.71 & $+10^{\circ}$ & 10.0 & 0.0415 & $-4.8^{\circ}$ & 1.001 & $0.31 \mathrm{PS}$ & 0.0387 & $.5 .17^{\circ}$ \\
\hline $16 \mathrm{~d}$ & 0.25 PS & 0.00445 & 0.71 & $+15^{\circ}$ & 10.0 & 0.1199 & $.8 .7^{\circ}$ & 0.998 & 0.31 PS & $\cdots$ & $-\cdot$ \\
\hline $17 \mathbf{a}$ & 0.25 PS & 0.00628 & 0.71 & $-10^{\circ}$ & 10.5 & 0.0324 & $-4.8^{\circ}$ & 0.997 & $-\cdot$ & 0.0300 & $-4.73^{\circ}$ \\
\hline $17 \mathrm{~b}$ & $0.25 \mathrm{PS}$ & 0.00628 & 0.71 & $\infty$ & 10.5 & 0.0389 & $-4.1^{\circ}$ & 0.997 & $0.27 \mathrm{sS}$ & 0.0314 & $.4 .74^{\circ}$ \\
\hline $17 \mathrm{c}$ & 0.25 PS & 0.00628 & 0.71 & $+10^{\circ}$ & 10.5 & 0.0434 & $-4.6^{\circ}$ & 0.999 & $0.10 \mathrm{SS}$ & 0.0395 & $-5.14^{\circ}$ \\
\hline $17 \mathrm{~d}$ & 0.25 PS & 0.00628 & 0.71 & $+15^{\circ}$ & 10.5 & 0.1539 & $-9.2^{\circ}$ & 0.996 & $0.09 \mathrm{SS}$ &.- & -. \\
\hline
\end{tabular}

SS $^{\circ}$ - Suction Surface Roughness

PS"• - Pressure Surface Roughness

Trans "- - Onset of Transition Point

\subsubsection{Blade Static Pressure Distribution}

Midspan static pressure measurements were obtained for design and off-design incidence angles with and without roughness applied to the blade surfaces. Of the many pressure distributions ibtaneci, a small portion covering the key aspects were selected for presentation in this section. 


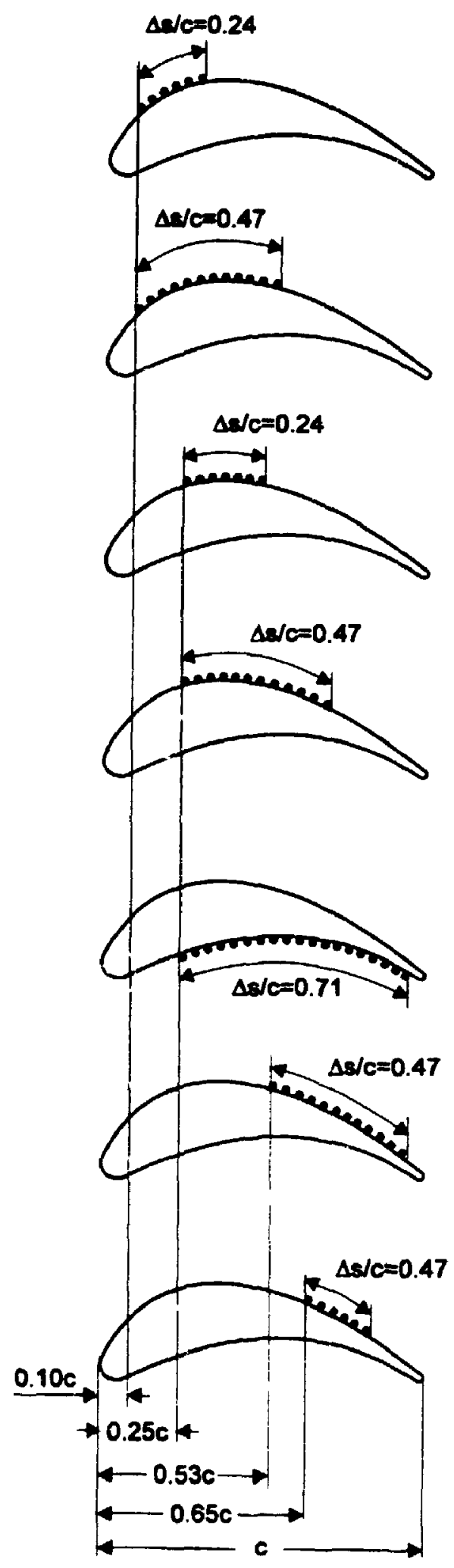

Figure 5.1: Locations and roughness band widths on the blade profile. 


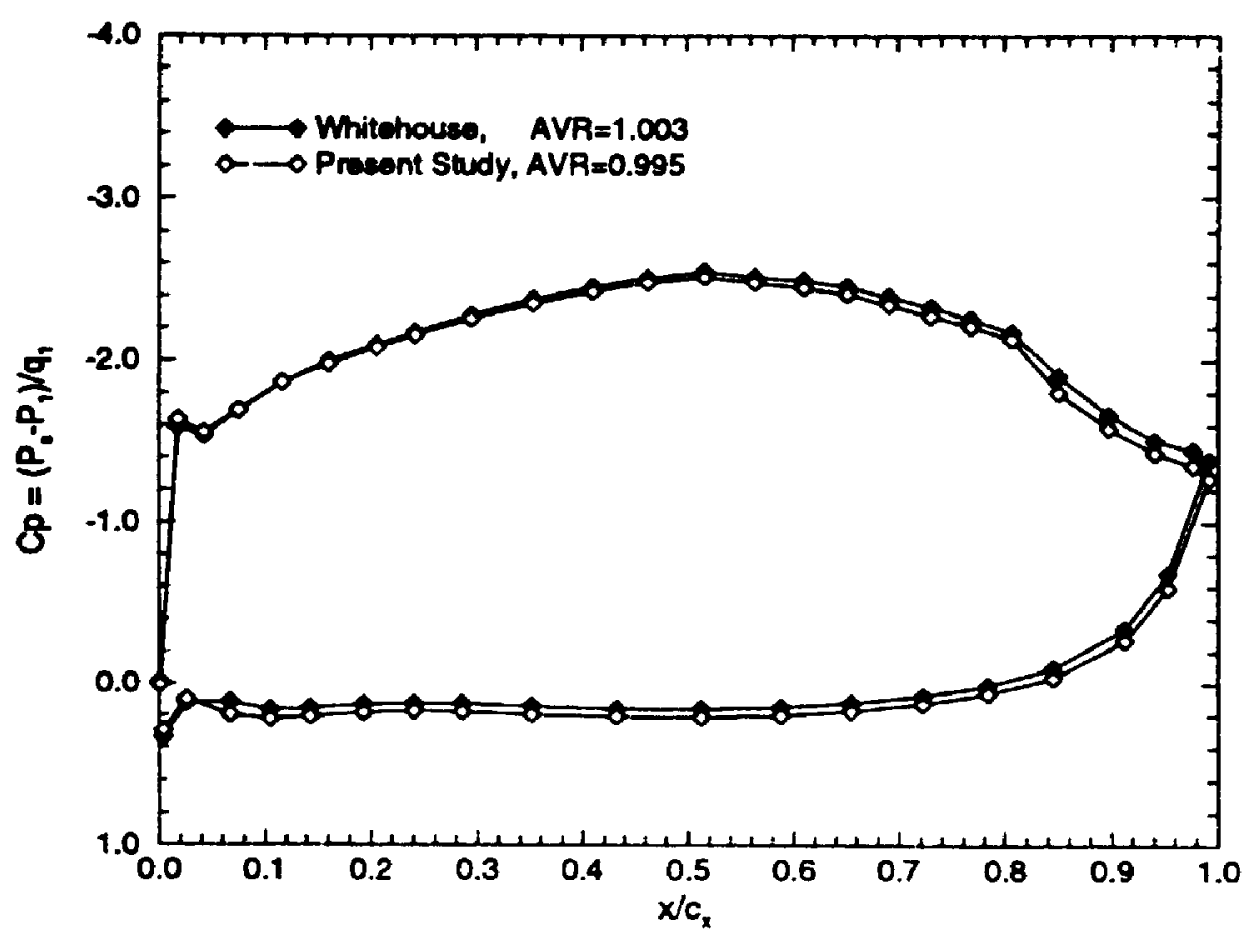

Figure 5.2: Midspan blade static pressure distributions at Casign incidence; comparison with Whitehouse (1993).

The initial tests jy the author attempted to reproduce the midspan static pressure measurements obtained by Whitehouse (1993). Whitehouse (1993) obtained midspan pressure distributions for a full range of incidence angles using the same cascade (i.e. CC3 blades). Figure 5.2 shows a comparison of the pressure distribution obtained by Whitehouse (1993) and one obtained from the present study at design incidence. As can be seen, there is good agreement between the two results, although there is a subtle difference over the last $20 \%$ of axial chord on the suction side. The discrepancy is possibly due to the difference in the axial velocity ratios (AVR) between the two results. The AVR values for both curves are given in the legend. Rodger (1992) showed that increasing the AVR resulted in higher blade loading on the rear part of the blade. 


\subsubsection{Rough-Surface Blades}

\subsection{Design Incidence}

Figure 5.3 shows a comparison between the pressure distributions obtained for smooth blades and two obtained with different surface roughness configurations for similar AVR values. As can be seen, the static pressure distributions are not greatly affected by the presence of the roughness. This coincides with the findings of Speidel (1954) and Bammert and Sandstede (1980). Trial 14a consists of medium size roughness with the roughness leading edge located at a $0.10 x / c$ and covering $0.47 \Delta s / c$ of the blades suction surface and Trial $17 \mathrm{~b}$ consist of large size roughness with the roughness leading edge located at $0.25 x / c$ and covering $0.71 \Delta s / c$ of the pressure surface.
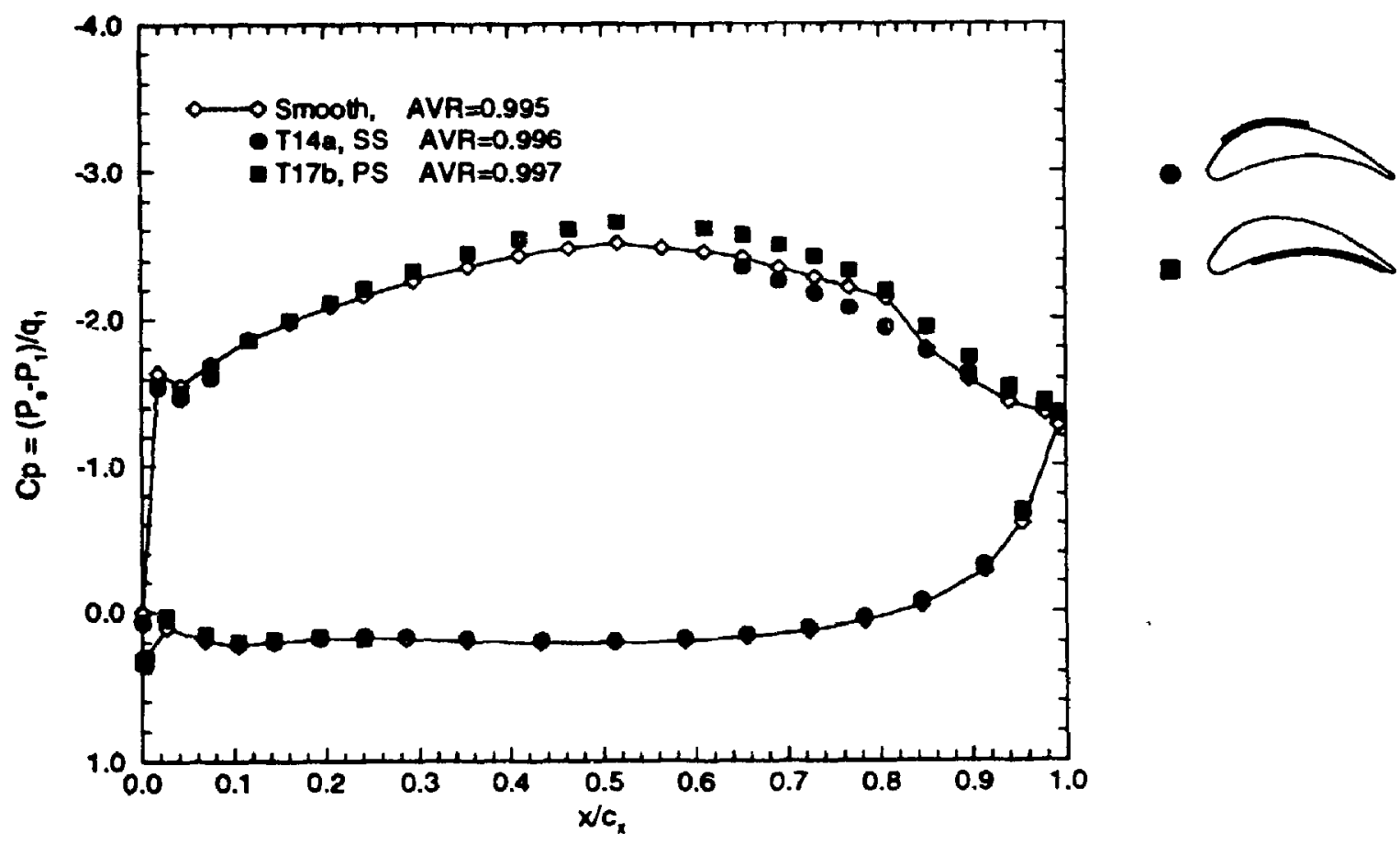

Figure 5.3: Midspan blade static pressure distributions with and without roughness applied to either the pressure and suction surfaces at design incidence. 
The roughness bands cover the local blade static taps, therefore these points are omitted in Figure 5.3. Although the presence of the roughness does not appear to greatly affect the static pressure distribution on the pressure surface, there is evidence of a change in blade loading on the suction surface for both Trials $14 \mathrm{a}$ and $17 \mathrm{~b}$.

The blade loading near the trailing edge is reduced with roughness applied to the suction surface (eg. T14a). Laminar to turbulent transition occurs naturally on the smooth blade cascade near mid chord. Measurements made using the stethoscope described in Section 3.3.7 showed that, with roughness, laminar to turbulent transition occurred near the leading edge of the roughness band. The roughness therefore triggers early transition and increases the boundary layer thickness over the remaining blade surface which in turn reduces the curvature of the streamlines near the trailing edge and hence reduces the suction and the blade loading.

Roughness applied to the pressure surface (e.g. T17b) once again triggers transition to a turbulent boundary layer thereby increasing the boundary layer thickness. This in turn reduces the effective passage width thus increasing the velocity of the core flow in the passage. The effect of the higher passage velocity is most evident on the suction surface, as seen in Figure 5.3. This is because pressures are dependent on the square of velocity and velocity on the suction surface is much higher than on the pressure surface. The effects are also somewhat evident in the corresponding flow deviation data, which will be discussed further in Section 5.1.2.

\subsection{Off-Design Incidence}

Figure 5.4 shows three pressure distributions obtained at $+15^{\circ}$ incidence. A smooth blade pressure distribution and two obtained with the roughness leading edge located at $0.10 x / c$ on the suction surface and covering $0.24 \Delta s / c$ and $0.47 \Delta s / c$ of the blades' surfaces. Once again, the pressure distribution is not greatly affected by the 
surface roughness, although there is slight evidence of a change in loading at the trailing edge on the suction surface. Although there is no available experimental data discerning whether the boundary layer separates from the blade surface near the trailing edge at $+15^{\circ}$ incidence, and the pressure distribution for the smooth blades shown in Figure 5.4 does not clearly show separated flow, experiments by Whitehouse (1993) examining the same cascade of blades at $+20^{\circ}$ incidence concluded, using flow visualization, that the aft portion of the blade suction surface did experience flow separation. The midspan loss measurements made at $+15^{\circ}$ and $+20^{\circ}$ angles of incidence are significantly higher than the values obtained at design incidence, which suggests the flow at $+15^{\circ}$ is subject to flow separation near the trailing edge. Examination of the pressure distribution with roughness applied to the suction surfaces shows evidence of the flow separation point moving forward with larger roughness band width thereby increasing the midspan total pressure loss.

Figure 5.5 shows three pressure distributions obtained at $+.0^{\circ}$ incidence. Trial $17 \mathrm{c}$ consists of large roughness with the leading edge of the roughness located at $0.25 x / c$ and covering $0.71 \Delta s / c$ of the pressure surface and Trial 14b consists of medium size roughness located at $0.10 x / c$ covering $0.47 \Delta s / c$ of the suction surface. As can be seen, roughness applied to the pressure surface does not greatly affect the pressure distribution at this incidence. Once again, measurements made using a stethoscope suggested laminar to turbulent transition on the pressure surface occurred near the leading edge of the roughness band. This would tend to increase the boundary layer thickness, although, due to the high positive incidence angle the increase in boundary layer thickness on the pressure side has relatively little affect on the passage velocity compared to roughness on the pressure surface at design incidence. Roughness applied to the suction surface reduces the loading at the trailing edge as it does for suction surface roughness at design incidence. 


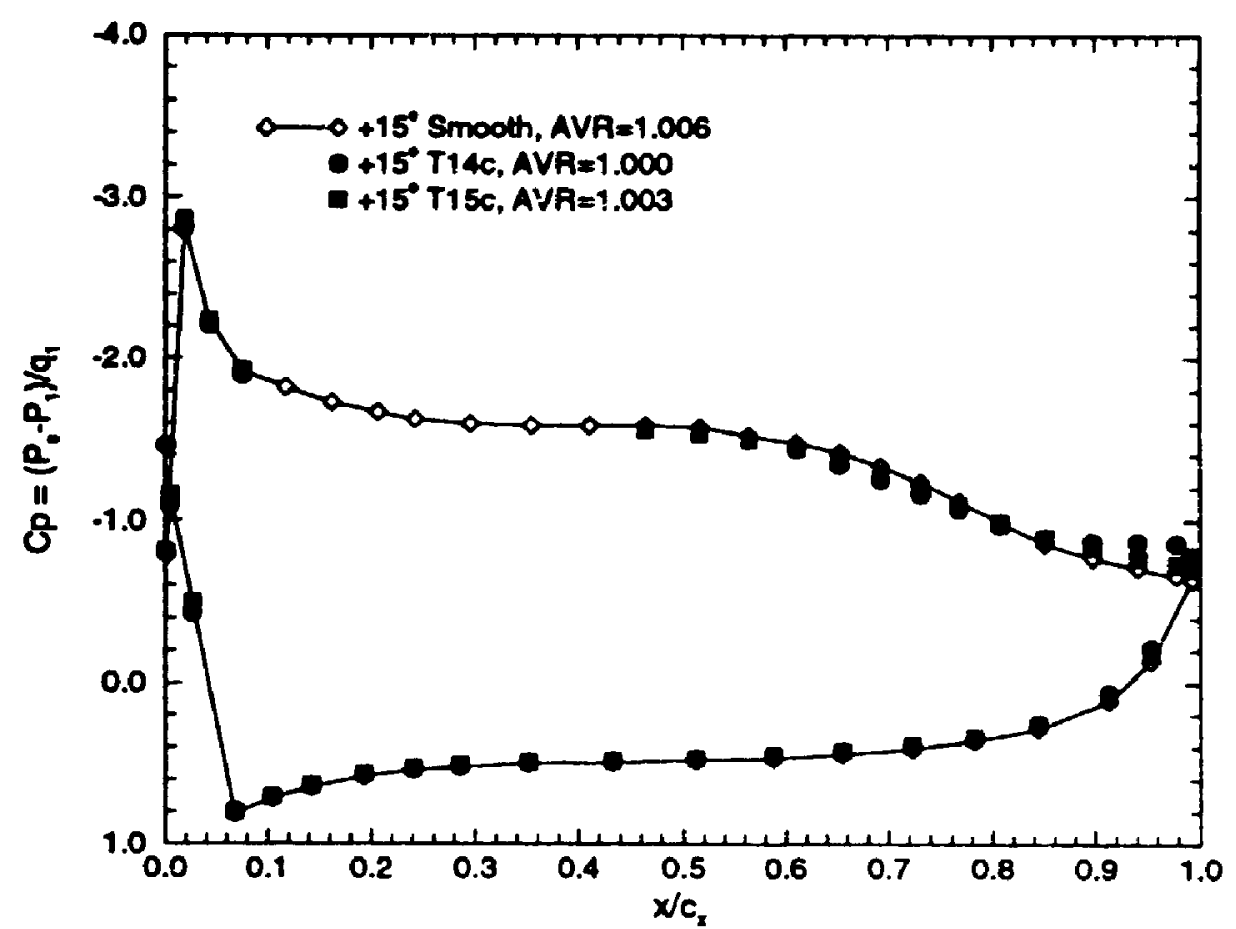

Figure 5.4: Midspan static pressure distribution with and without roughness applied to the suction surface at $+15^{\circ}$ incidence.
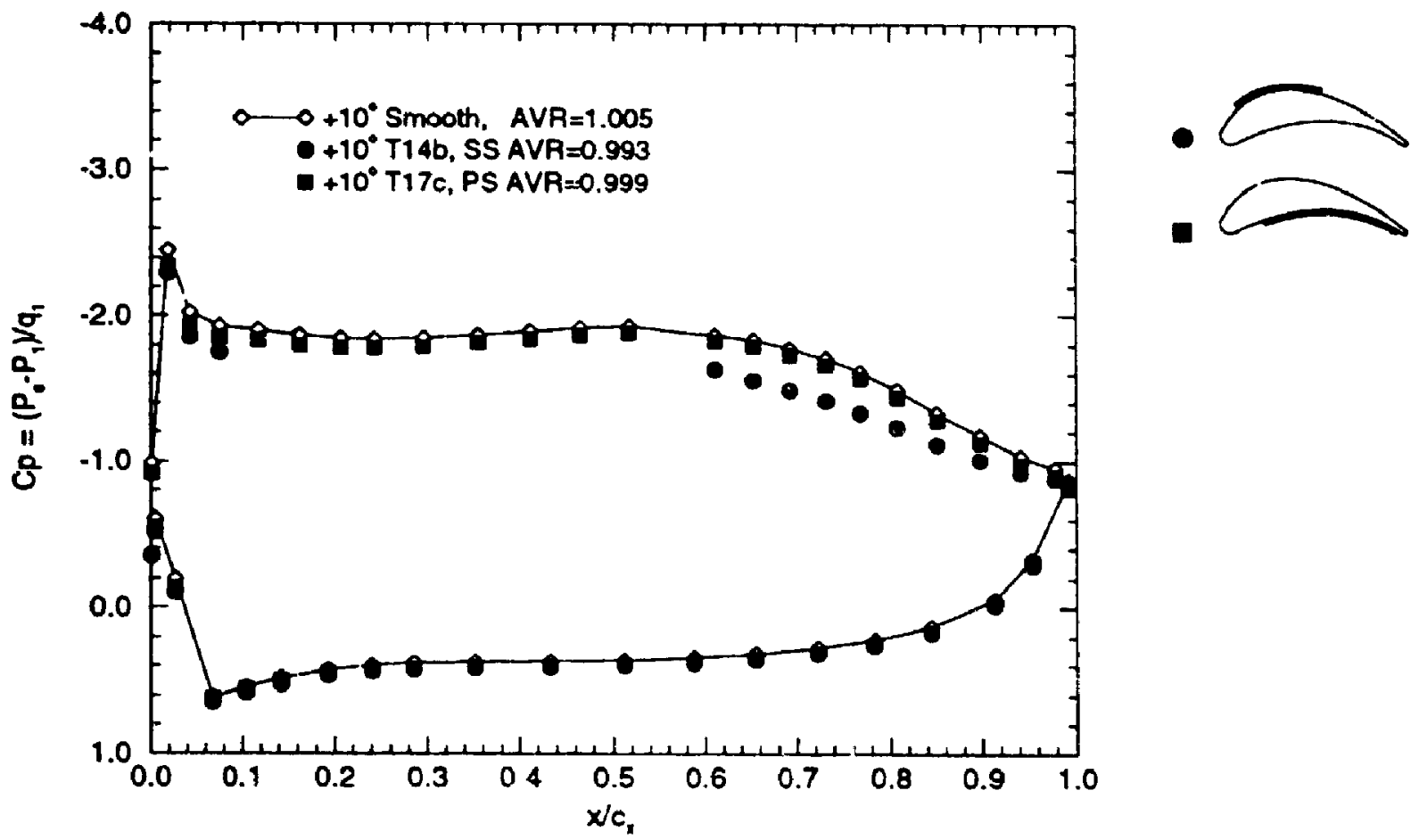

Figure 5.5: Midspan blade static pressure distribution with and without roughness applied to the suction and pressure surface at $+10^{\circ}$ incidence. 

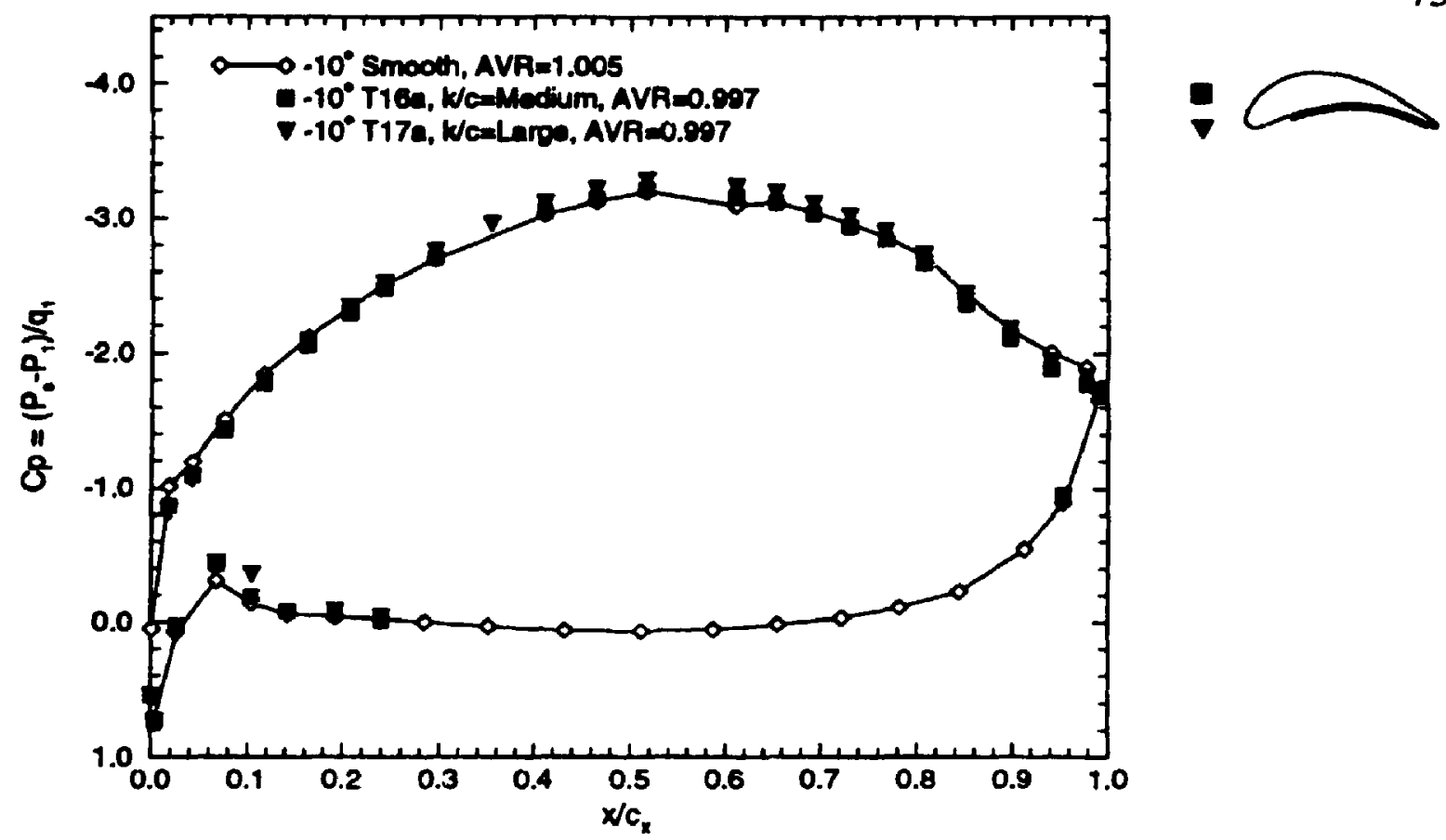

Figure 5.6: Midspan blade static pressure distribution with various roughness sizes applied to the pressure surface at $-10^{\circ}$ incidence.

Figure 5.6 shows the pressure distributions obtained at $-10^{\circ}$ incidence. For both Trial 16a and 17a the roughness leading edge is located at $0.25 x / c$ and it covers approximately $0.71 \Delta s / c$ of the pressure surface and the roughness is of medium size and large size respectively. A: $-10^{\circ}$ incidence a definite increase in the blade loading on the suction side is evident with increasing roughness height

\subsubsection{Total-Pressure Loss and Flow Deviation}

\subsubsection{Smooth Blades}

The initial tests by the author attempted to reproduce the off-design loss data obtained by Whitehouse (1993) and Abbott (1993) for the same cascade (i.e. CC3). 
Figures 5.7 and 5.8 shows the comparisons of total-pressure loss and flow deviation respectively. As shown, the present study was limited to a data range between $-10.0^{\circ}$ to $+15.0^{\circ}$ effective incidence.

The data for total-pressure loss, $Y_{m}$. compare very well except at the higher incidence angles. The difference in loss at $+15.0^{\circ}$ between Whitehouse (1993) and the present study and at $+20.0^{\circ}$ between Whitehouse (1993) and Abbott (1993) may be attributed to a difference in the axial velocity ratio (AVR) (at $+15.0^{\circ}$ Whitehouse: $A$ VR= 1.009, present study: $A V R=1.002$, at $+20.0^{\circ}$ Whitehouse: $A V R=0.985$, Abbott: $A V R=$ 1.031). Whitehouse (1993) showed a very strong sensitivity to AVR at higher incidence angles. As the AVR is increased past unity, a gradual reduction in the midspan loss was observed. This was linked to the effect of the flow acceleration on the position of flow separation. An increase in AVR would tend to move the separation point toward the blade trailing edge and reduce the losses.

The deviation angle, $\delta$, shown in Figure 5.8, does not compare as well as the totalpressure loss data, although the trends are similar from the present data and those of Whitehouse (1993) and Abbott (1993). The difference between the present deviation angle data and that of Whitehouse (1993) and Abbott (1993) is in the order of $1^{\circ}$. The discrepancies can be attributed to the accuracy of the three-hole probes. as discussed in Section 3.2.2.2. 


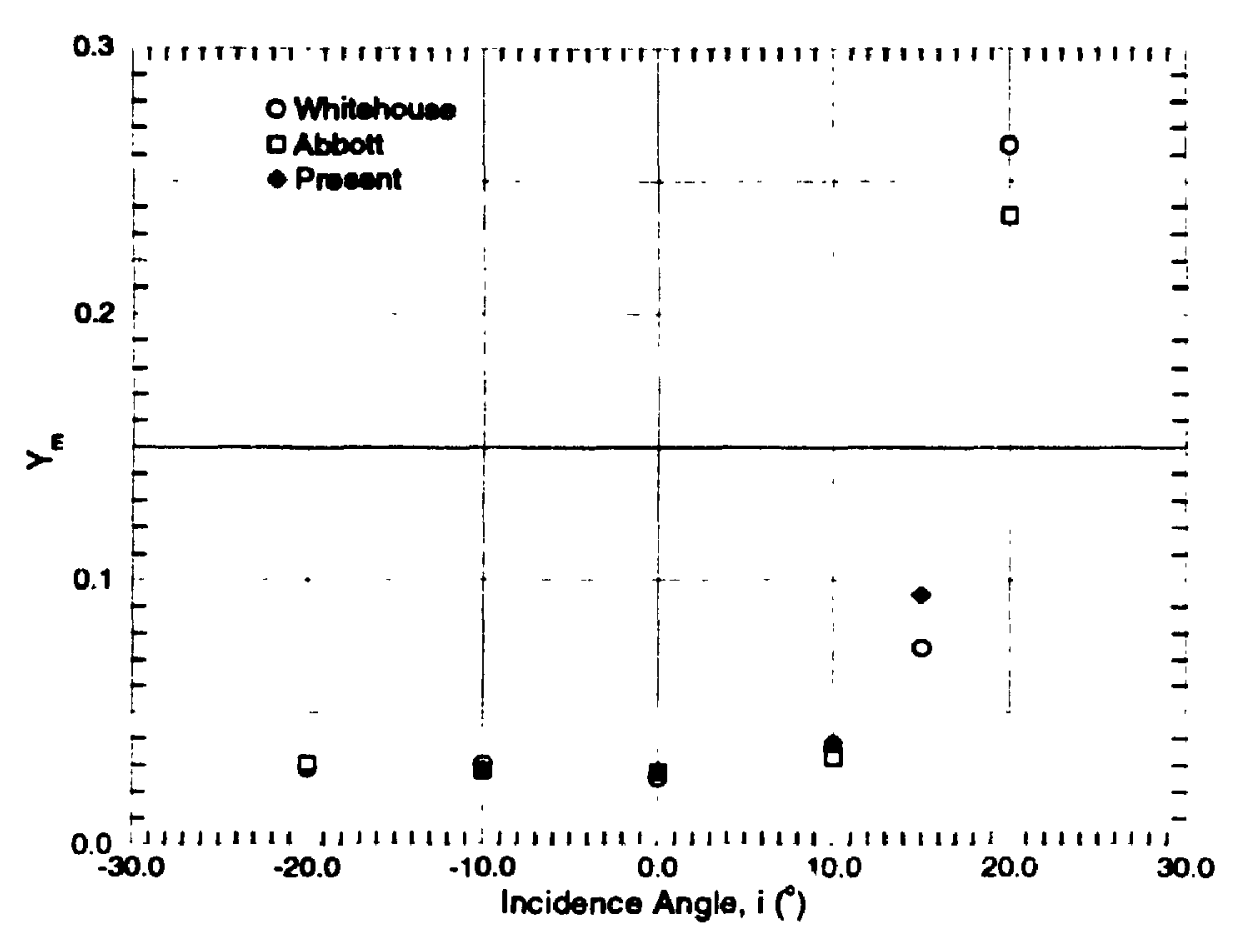

Figure 5.7: Total-pressure-loss coefficient versus incidence for smooth blades; comparison with Whitehouse (1993) and Abbott (1993).

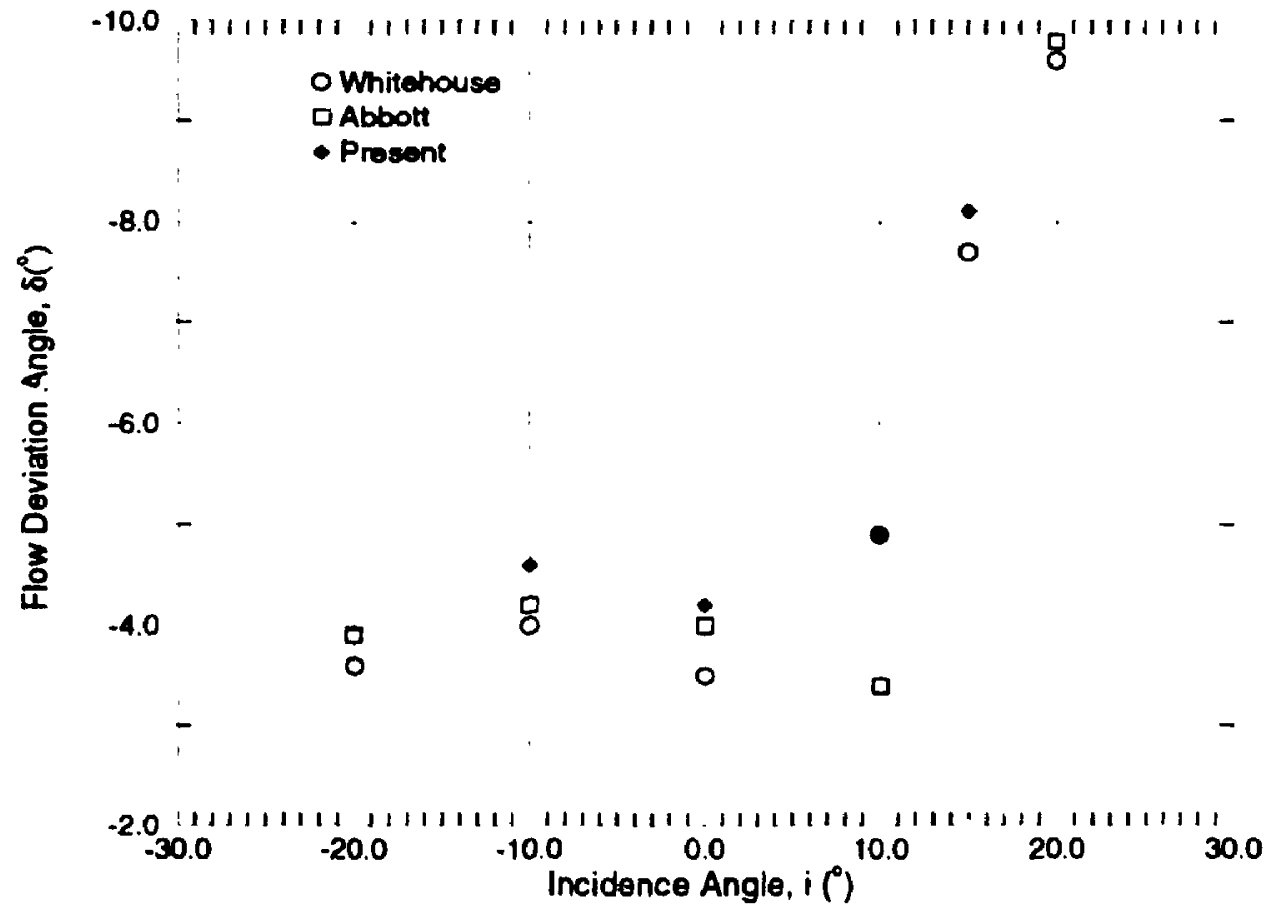

Figure 5.8: Flow deviation angle versus incidence for smooth blades; comparison with Whitehouse (1993) and Abbott (1993). 


\subsubsection{Effect of Roughness}

Prior to investigating new roughness configurations, attempts were made by this author to reproduce the data of Abbott (1993) for three specific roughness configurations at design incidence; namely, Trial 9a, 10a and 11a.

Figure 5.9 shows a comparison between Abbott (1993, and the present study of the total-pressure loss for two different roughness sizes and two different roughness band widths at design incidence. The present data shown in Figure 5.9 indicates that for the most part the loss measurements were consistent with the data of Abbott (1993). Although, with large roughness height (i.e. $k / c=0.00628$ ) and large band width the difference between the two values was found to be the greatest (i.e. $8 \%$ ). Examination of the spacing parameter, $\lambda$, showed that it differed by approximately $55 \%$ between the two cases and this may well be the reason for the discrepancy.

The deviation angle data, corresponding to the pressure-loss data shown in Figure 5.9, are given in Figure 5.10. The data of Abbott (1993) agree well with the present data. although there is some discrepancy once again with the large rough. iess height and large band width case. This may be attributed to the large ditterence i the spacing parameter. Abbott (1993) noticed the same trends in pressure loss and deviation when investigating the repeatability of his data for Trial $7 \mathrm{a}$ and Trial 11 a for different values of the spacing parameter.

\subsection{Design Incidence}

Figure 5.11 shows the vanation of total-pressure loss data from the present study with roughness height for various roughness band widths and locations. The legend of Figure 5.11 shows the location and width of the roughness bands with the corresponding 

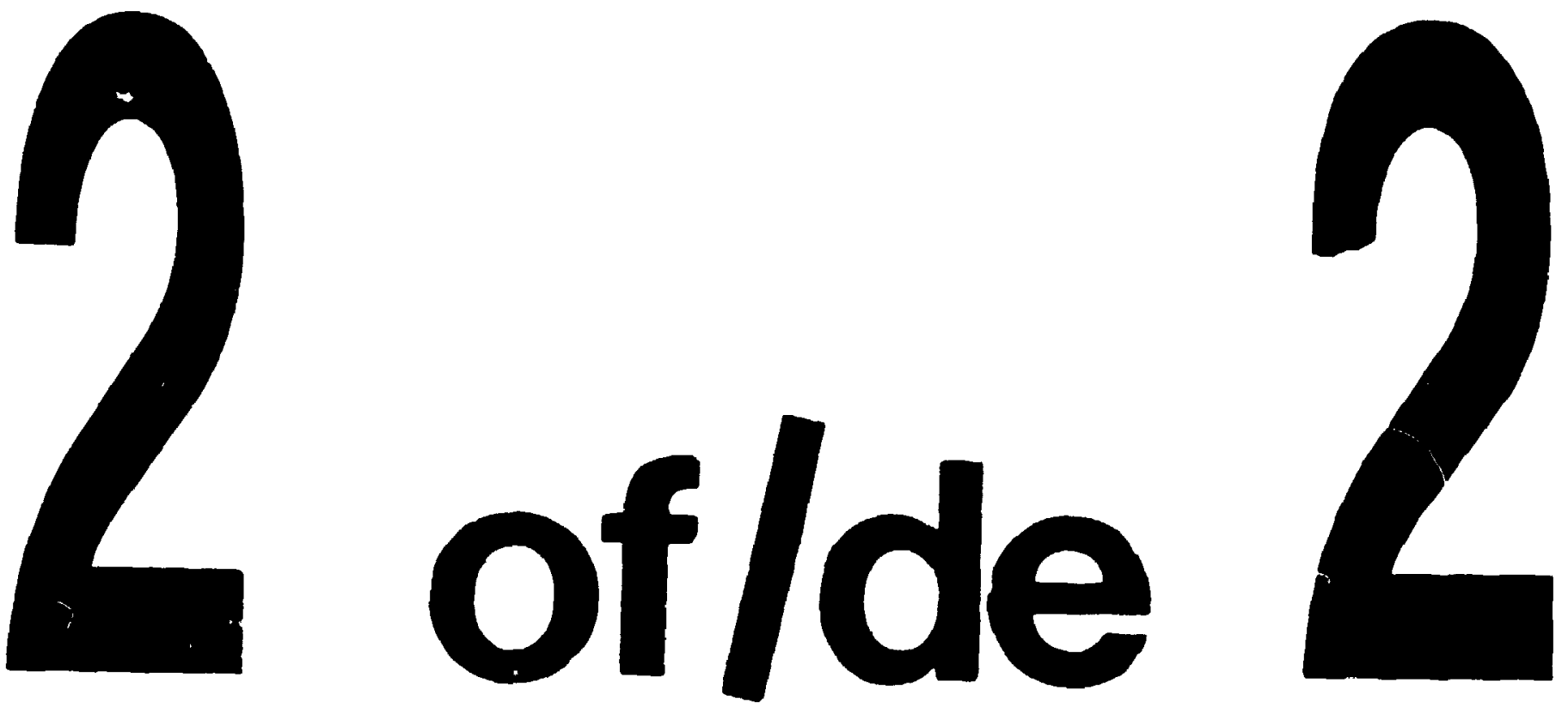

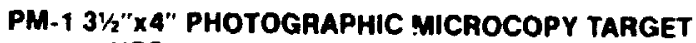
NBS 1010a ANSI/ISO \#2 EQUIVALENT

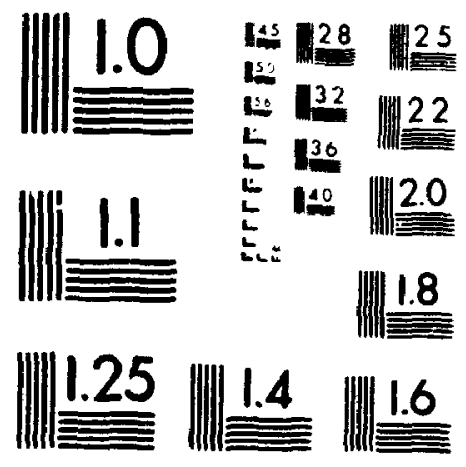

PRECISIONSM RESOLUTION TARGETS 


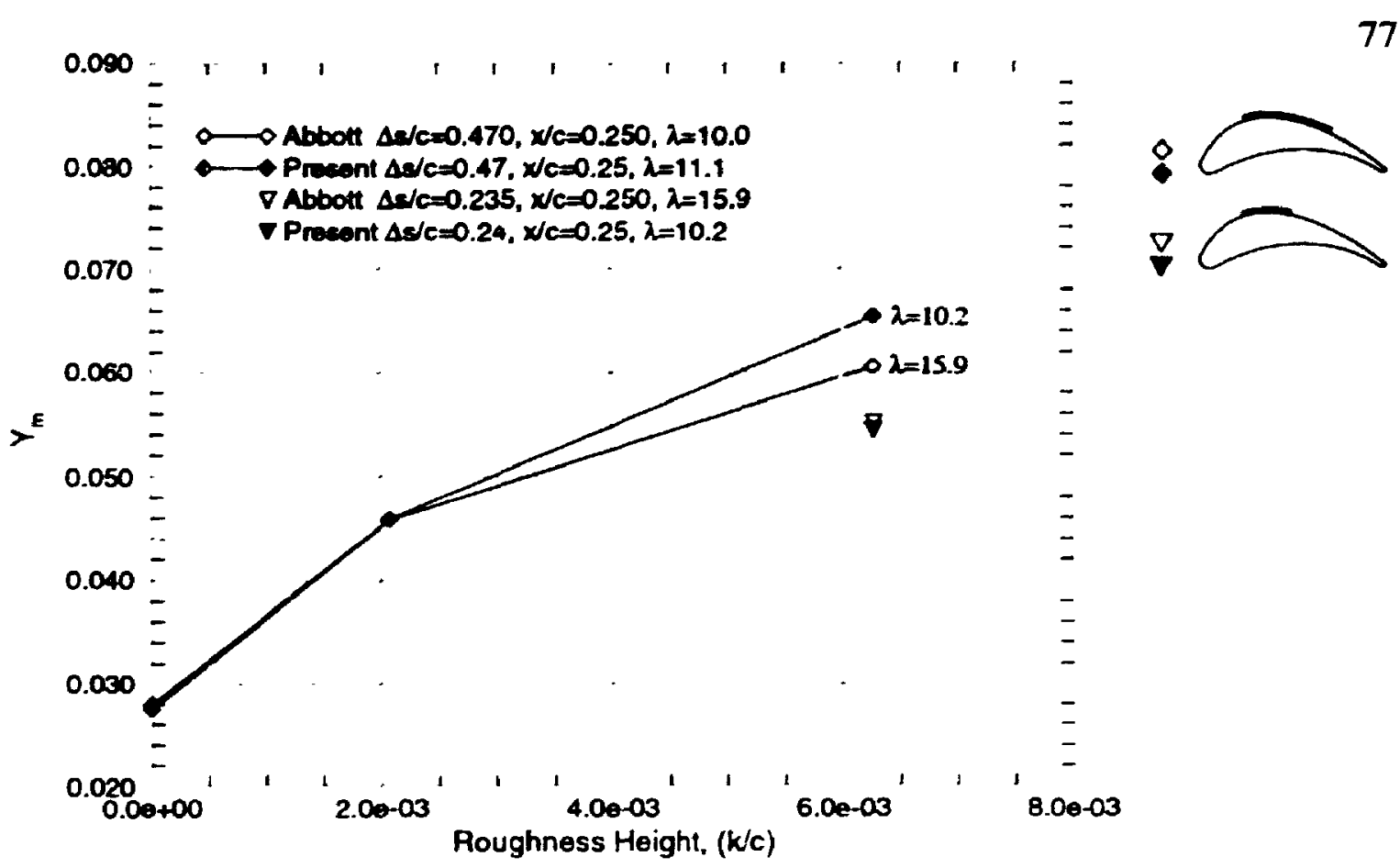

Figure 5.9: Total-pressure-loss coefficient versus roughness height, for design incidence; comparison with Abbott (1993).

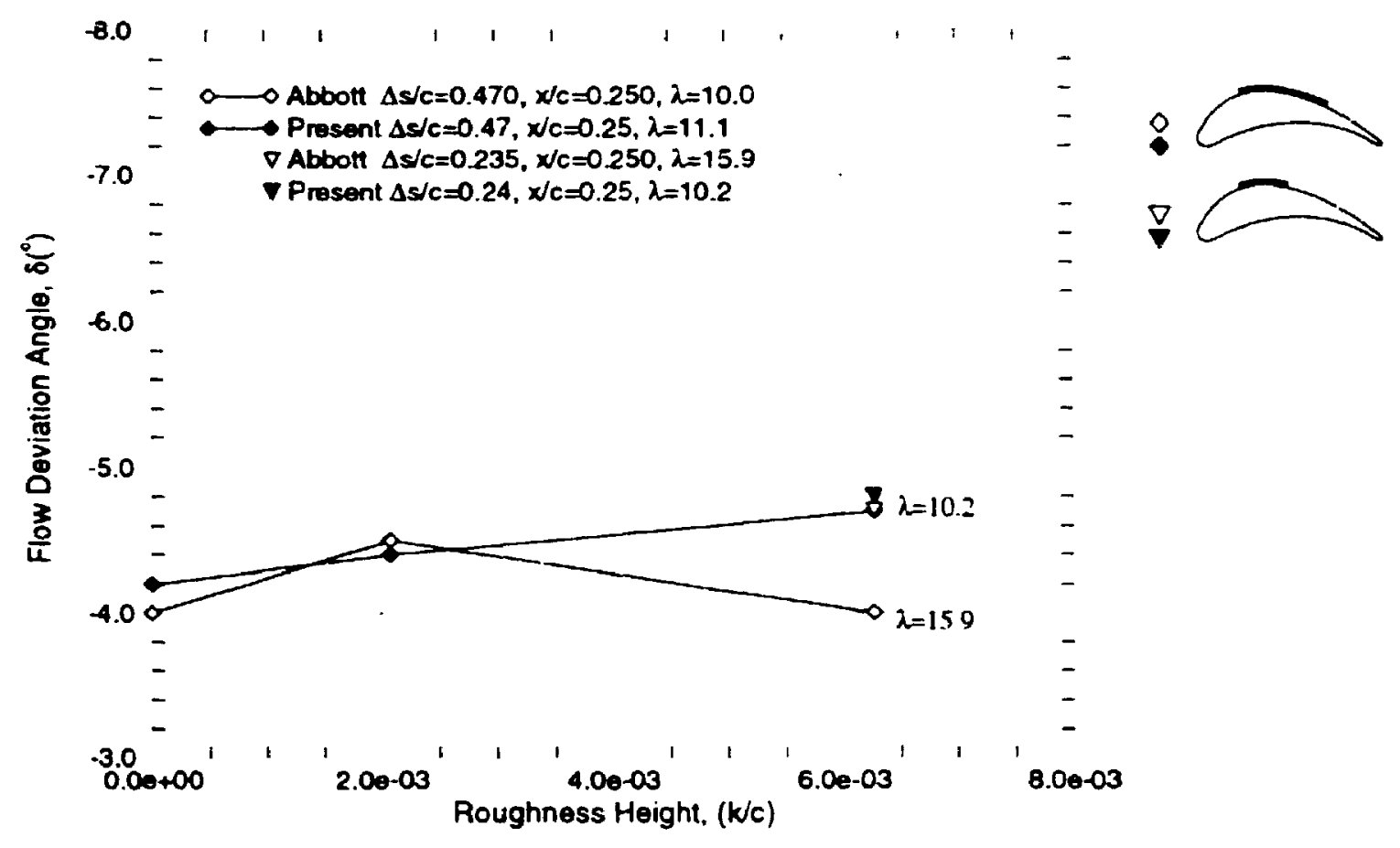

Figure 5.10: Flow deviation angle versus roughness height, for design incidence; comparison with Abbott (1993). 


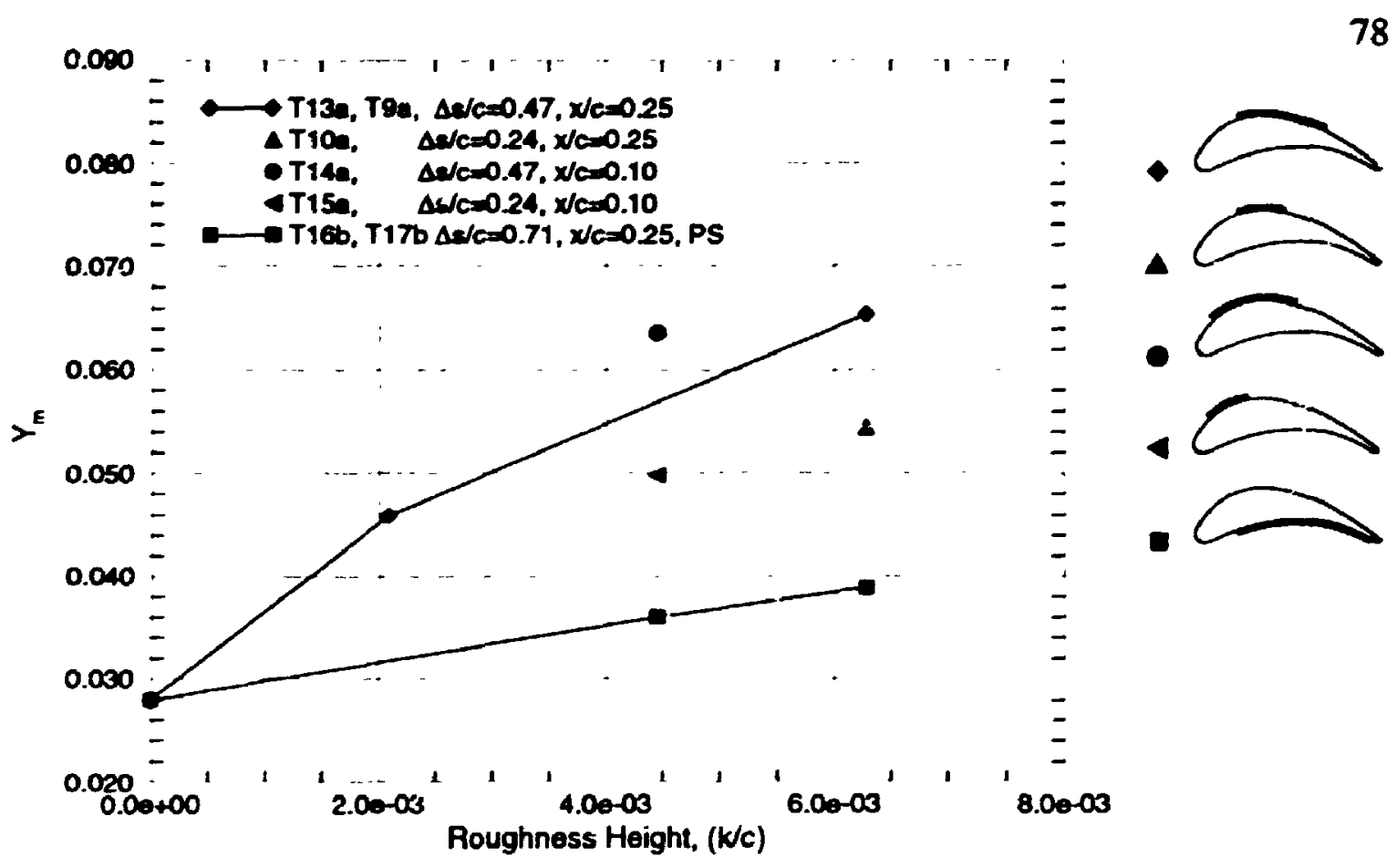

Figure 5.11: Total-pressure-loss coefficient versus roughness height, for design incidence.

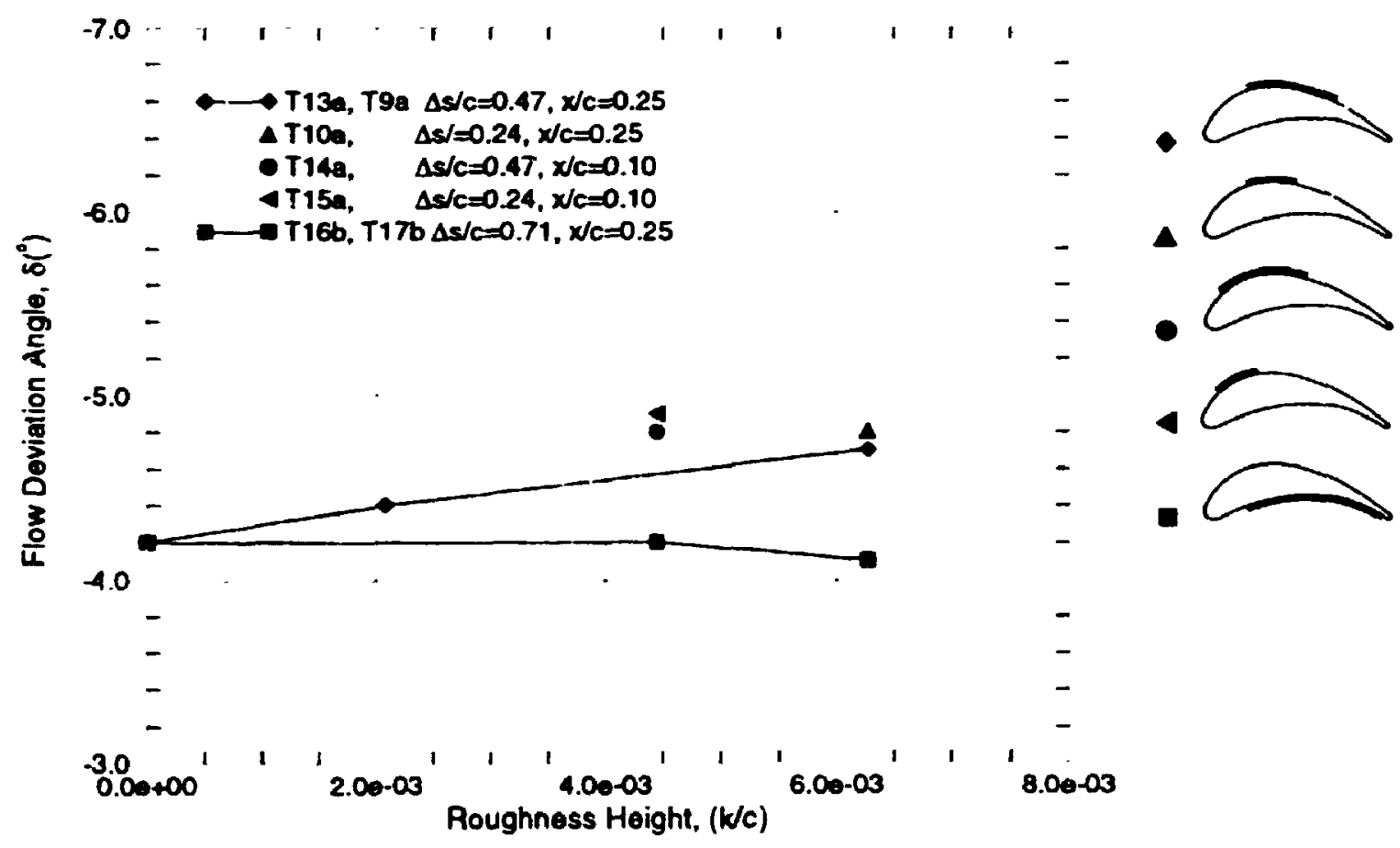

Figure 5.12: Flow deviation angle versus roughness height, for design incidence. 
trial numbers from Table 5.1. It is evident from Trials $13 \mathrm{a}$ and $9 \mathrm{a}$, and Trials $16 \mathrm{~b}$ and $17 \mathrm{~b}$, that the total-pressure loss increases approximately linearly with increasing roughness height. Abbott (1993) showed the same linear trend for different roughness configurations. Therefore, in an effort to reduce the number of experimental investigations, single data points are used to show the shift in total-pressure loss and a linear trend is assumed through the points.

Trial 14a, with the roughness leading edge located at $0.10 x / c$, and covering 0.47 $\Delta s / c$ of the blades' suction surface resulted in the largest increase in total-pressure loss. Roughness applied over $0.71 \Delta s / c$ of the pressure surface resulted in the smallest increase in loss.

Figure 5.12 shows the variation cî the outlet-flow deviation angle for different roughness heights corresponding to the pressure-loss values shown in Figure 5.11. A small increase in the deviation angle with roughness height is evident for roughness applied to the suction surface. Very small changes in the deviation angle were recorded with roughness applied to the pressure surface; a slight decrease in the deviation angle was recorded for the large roughness sample. The decrease in the deviation angle supports the increase in the blade loading on the sirtion surface shown in Figure 5.3. Nonetheless, the variation in the outlet-flow deviation angle is less than $1^{\circ}$ for all the cases shown. As stated previously, the accuracy to which the probe can be aligned is $\pm 0.5^{\circ}$.

\subsection{Off-Design Incidence}

Figures 5.13 and 5.14 show the losses for various roughriess configurations at offdesign incidence angles. Figure 5.13 shows the change in loss, $Y_{m}$, when the width of the roughness band is changed for roughness on the suction surface. The leading edge of the 
roughness bands are located at $0.10 x / c$ for both Trials 14 and 15 . The width of the roughness band for Trial 15 is $0.24 \Delta s / c$, half of Trial 14. The curves show that increasing the width of the roughness band causes a uniform increase in loss. This is consistent with the findings of Abbott (1993) for different roughness locations. Figure 5.14 shows the change in the loss curves with roughness applict to the pressure surface and the suction surface. The curves for Trials 9 and 10 are for large size roughness applied to the suction surface with the leading edge of the roughness band located at 0.25 $x / c$. The width of the roughness band for Trial 10 is half of Trial 9. Once again, a uniform increase in loss is observed for a larger roughness band width. Medium and large size roughness were applied to $0.71 \Delta s / c$ of the pressure surface with the leading edge of the roughness band located at $0.25 x / c$. As shown by Trial 16 and 17 the roughness applied to the pressure surface causes a slight increase in loss at $-10^{\circ}, 0^{\circ}$ and $+10^{\circ}$ incidence angle, whereas at an incidence angle of $+15^{\circ}$ the loss increase is significant. A portion of the increase in loss can be attributed to the lower AVR values measured for Trials $16 \mathrm{~d}$ and $17 \mathrm{~d}$ compared to the smooth case. The presence of the roughness on the pressure surface may cause an acceleration of the freestream flow near the trailing edge on the suction surface sufficiently to cause the separation point to move forward increasing the separated region thereby increasing the losses.

The effect of roughness on the deviation angle is shown in Figure 5.15 and 5.16 corresponding to the loss data presented in Figure 5.13 and 5.14. The presence of roughness on the suction surface increases the deviation of the flow for all cases shown. For clarity, only Trial 9 and 17 are shown in Figure 5.16. Large roughness applied to the pressure surface appears to cause a slight decrease in the deviation angle for $0^{\circ}$ and $+10^{\circ}$ incidence angles, as compared to smooth blades. 


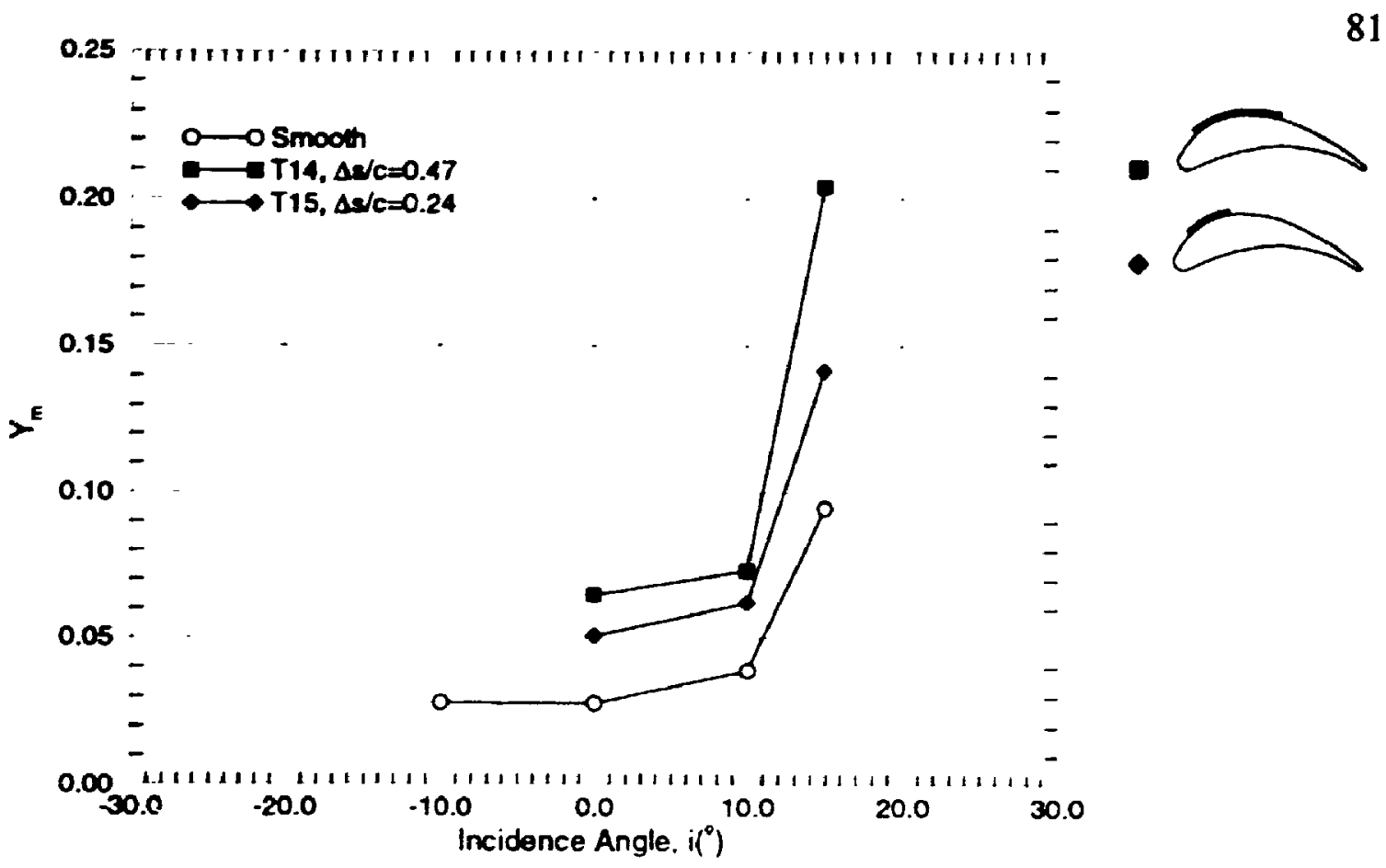

Figure 5.13: Total-pressure-loss coefficient versus incidence for various roughness band widths on the suction surface (with $k / c=0.00445$ (medium), $L E=0.10$ $\mathbf{x} / \mathbf{c})$.

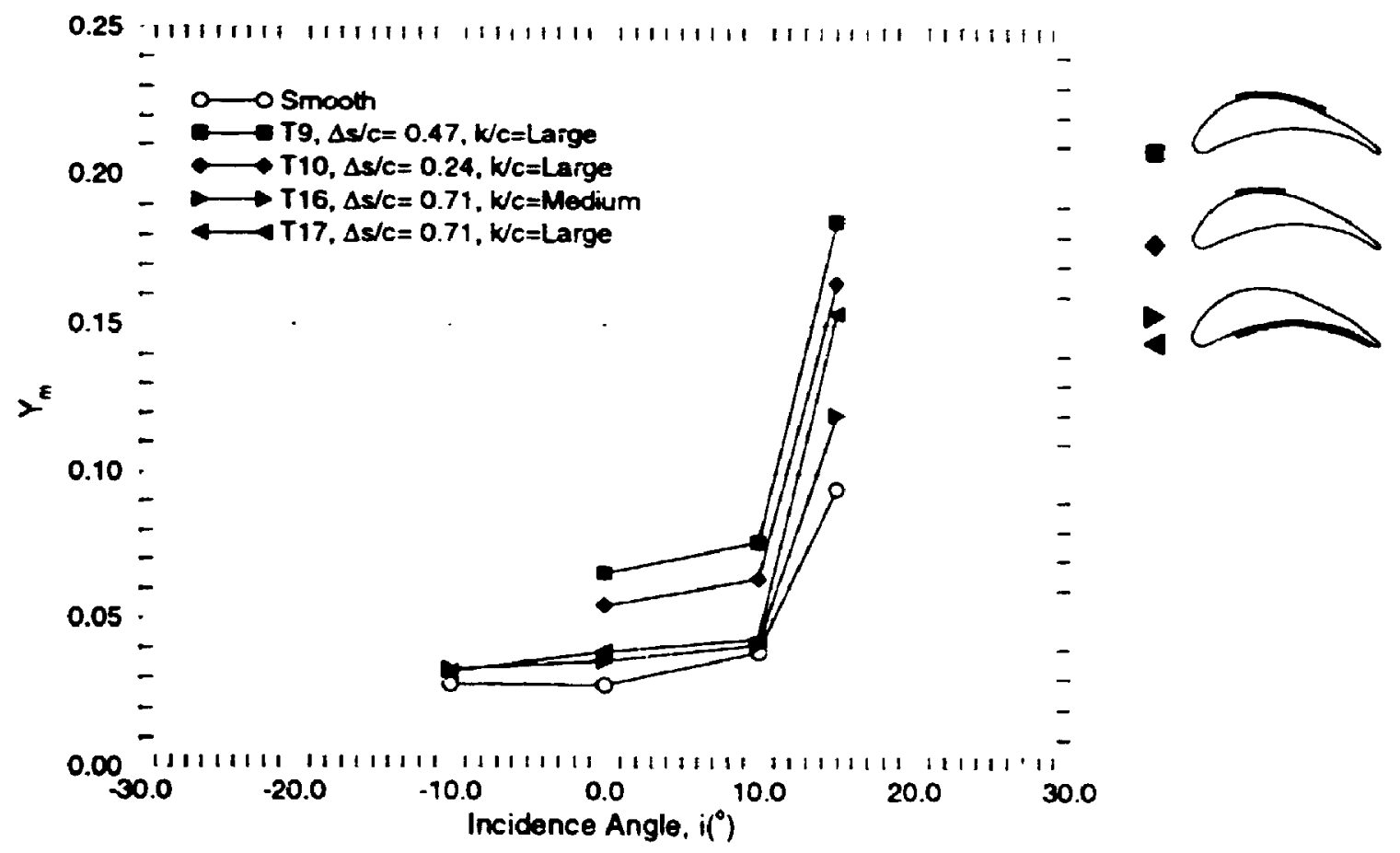

Figure 5.14: Total-pressure-loss coefficient versus incidence for various roughness configurations applied to the suction and pressure surface (LE $=0.25 \mathrm{x} / \mathrm{c}$ ). 


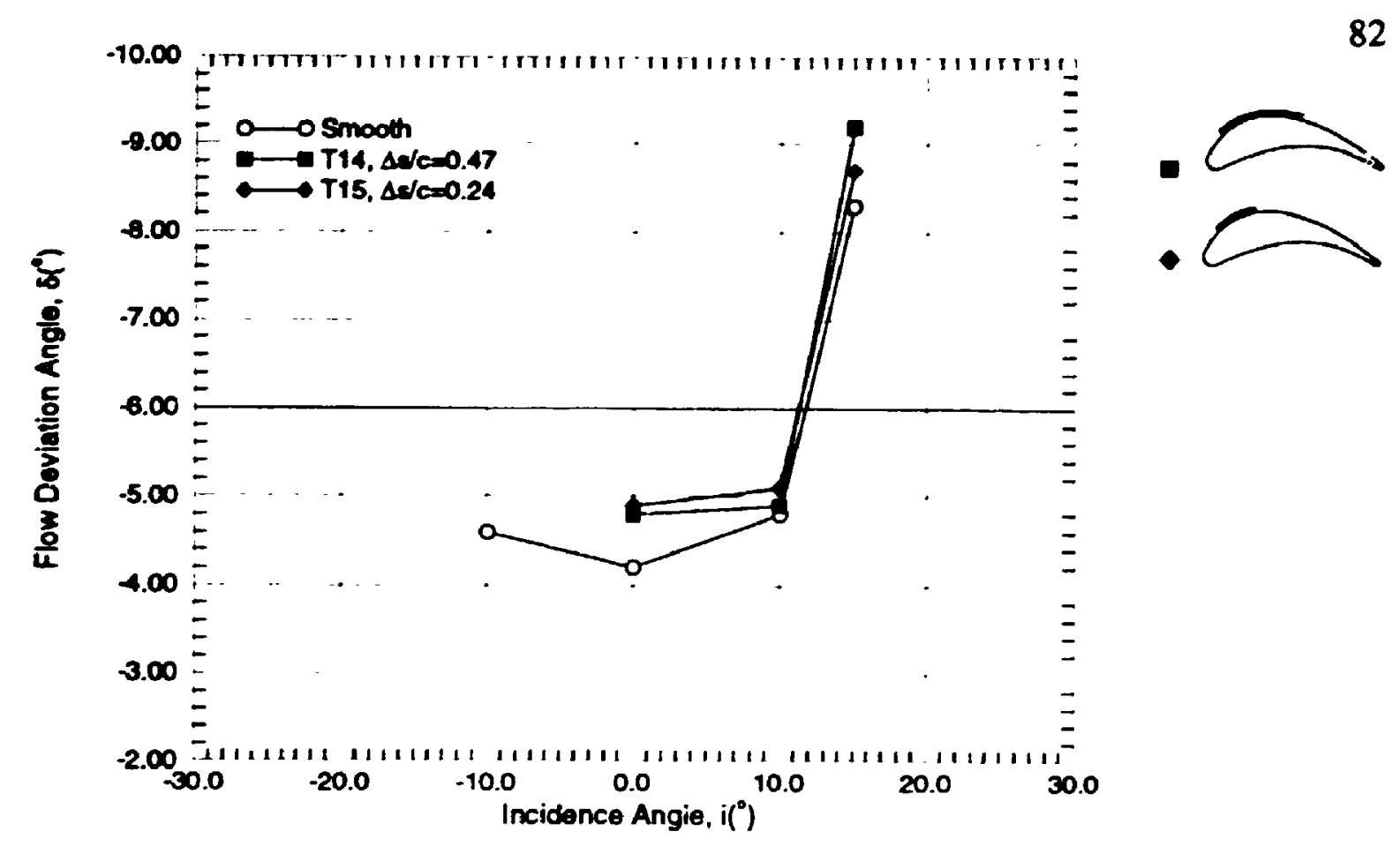

Figure 5.15: Flow deviation angle versus incidence for various roughness band widths on the suction surface (with $k / c=0.00445$ (medium), $L E=0.10 \mathrm{x} / \mathrm{c}$ ).

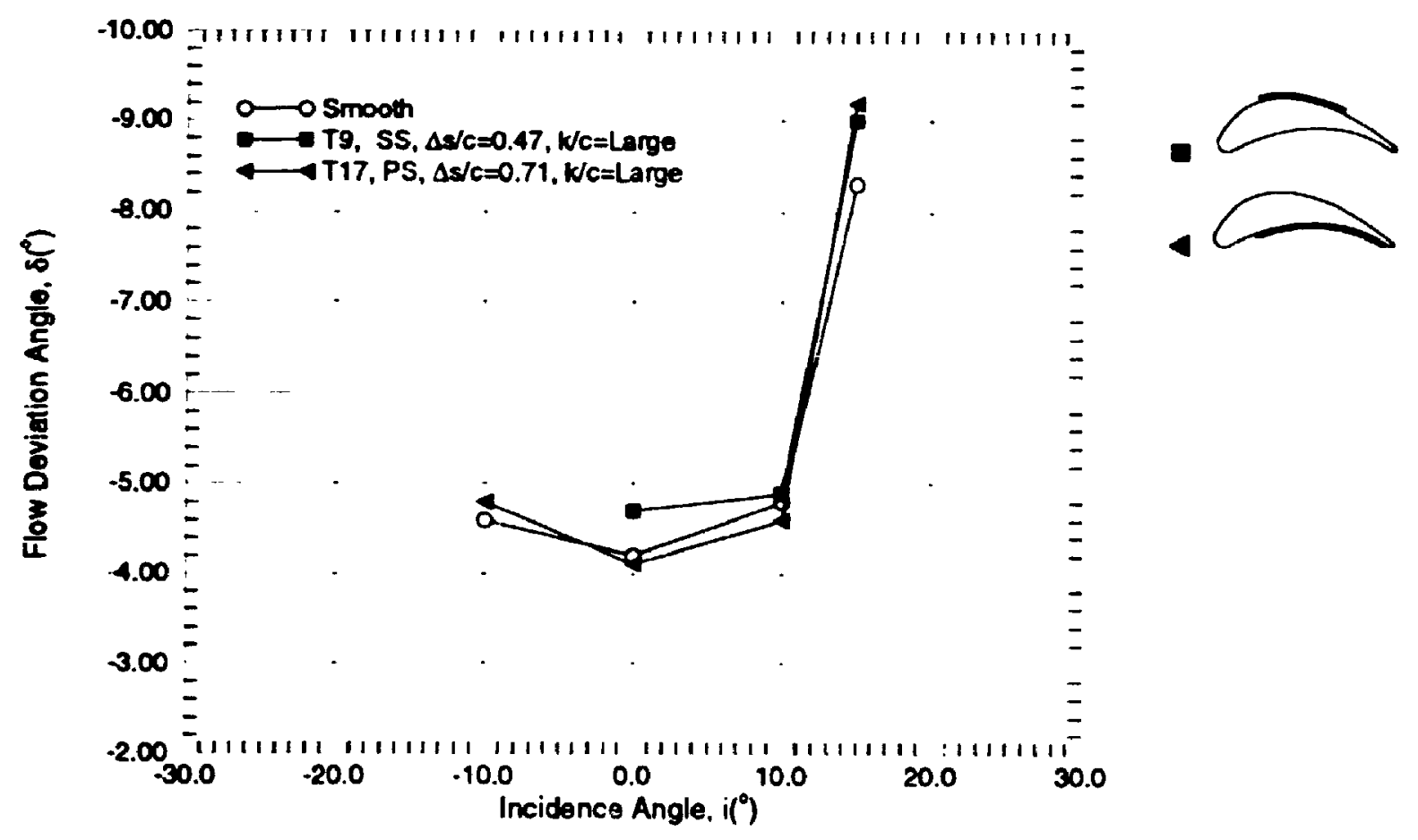

Figure 5.16: Flow deviation angle versus incidence for various roughness configurations applied to the suction and pressure surface (LE $=0.25 \mathrm{x} / \mathrm{c}$ ). 


\subsection{Computational Results}

\subsubsection{Validation of the Inviscid Solution}

The inviscid solution method was checked with Gostellow's (1984) exact solution for a cusped-profile cascade with the purpose of checking the predictions of the pressure distribution and outlet angle. Figure 5.17 shows the Gostellow cusped-profile and the corresponding pressure distributions. The nomenclature for the input parameters shown are provided in Chapter 4, Figure 4.4. The third form of the Kutta condition as described in Section 4.2.2 was used in the analysis. The pressure coefficients provided by Gostellow's exact solution are based on inlet conditions. therefore the results from the computational predictions were post processed as per the method described in Section 4.3.1. As shown in Figure 5.17, the pressure distribution determined by the inviscid solution agrees very well with Gostellow's exact solution and the predicted outflow arigle is within $0.15^{\circ}$ of the exact sulution.

\subsubsection{Computational Results and Comparison with Experimental}

As was the case with the experimental data, not all the data are presented in the following section. A small portion cf the data covering the key aspects is presented.

\subsubsection{Static Pressure Distribution}

\subsection{Smooth Blades}

Figure 5.18 shows the modified blade profile used in this study along with the computationally predicted and experimentally determined midspan pressure distributions 

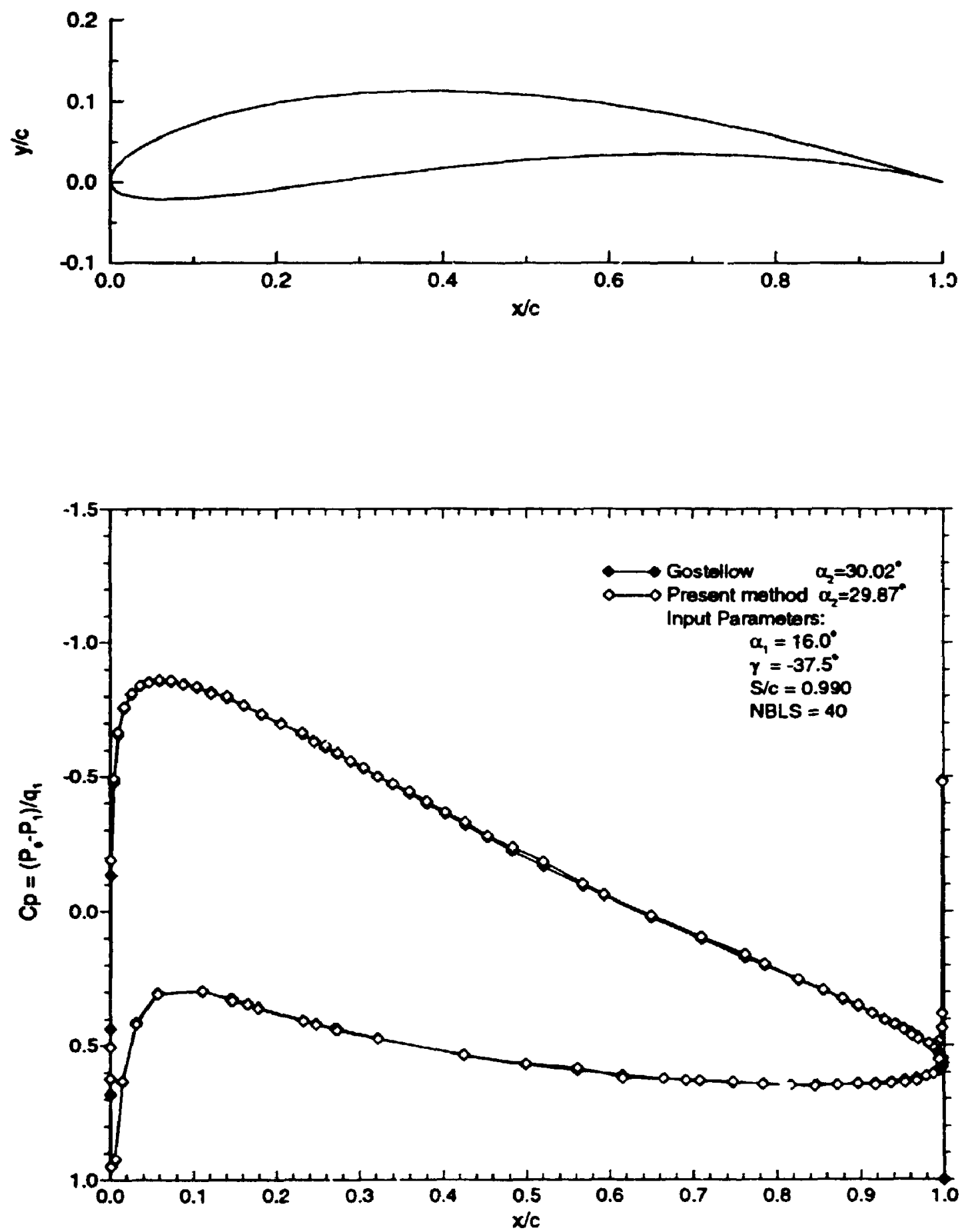

Figure 5.17: Pressure distributions and blade profile for the Gos :llow cusped blade cascade; comparison with inviscid solution. 
at design incidence for a smooth surface cascade. Consider the pressure distributions determined by the computational method. As can be seen, the boundary layer growth reduces the loading on the suction surface progressively from the leading edge to the trailing edge. The pressure side boundary layer effects are negligible. There is good agreement between the experimental and computational results, except near the leading and trailing edges.

The overspeeds at the leading edge predicted by the computational method tend to be somewhat larger than those in the experimental data. This may be due to a small separation bubble occurring near the leading edge on both the pressure and suction surfaces that is not considered in the computational model. As discussed in Section 4.2.3.2 the local velocities predicted by the inviscid computations were manipulated such that the boundary layer analysis did not erroneously predict separation or transition at these points.

Near the trailing edge there is less agreement between the measured pressure distribution and the computationally predicted pressure distribution. in particular the base pressure values differ by more than $40 \%$. The lower base pressure value measured experimentally is likely due to the small separated region developed on the rounded trailing edge. The separated region downstream of the trailing edge creates a low pressure region which is not modelled computationally. Although there is only modest agreement between the computational and experimental pressure distributions at the trailing edge, there is no evidence of velocity spikes which would occur if a blade with a rounded urailing edge was modelled. 

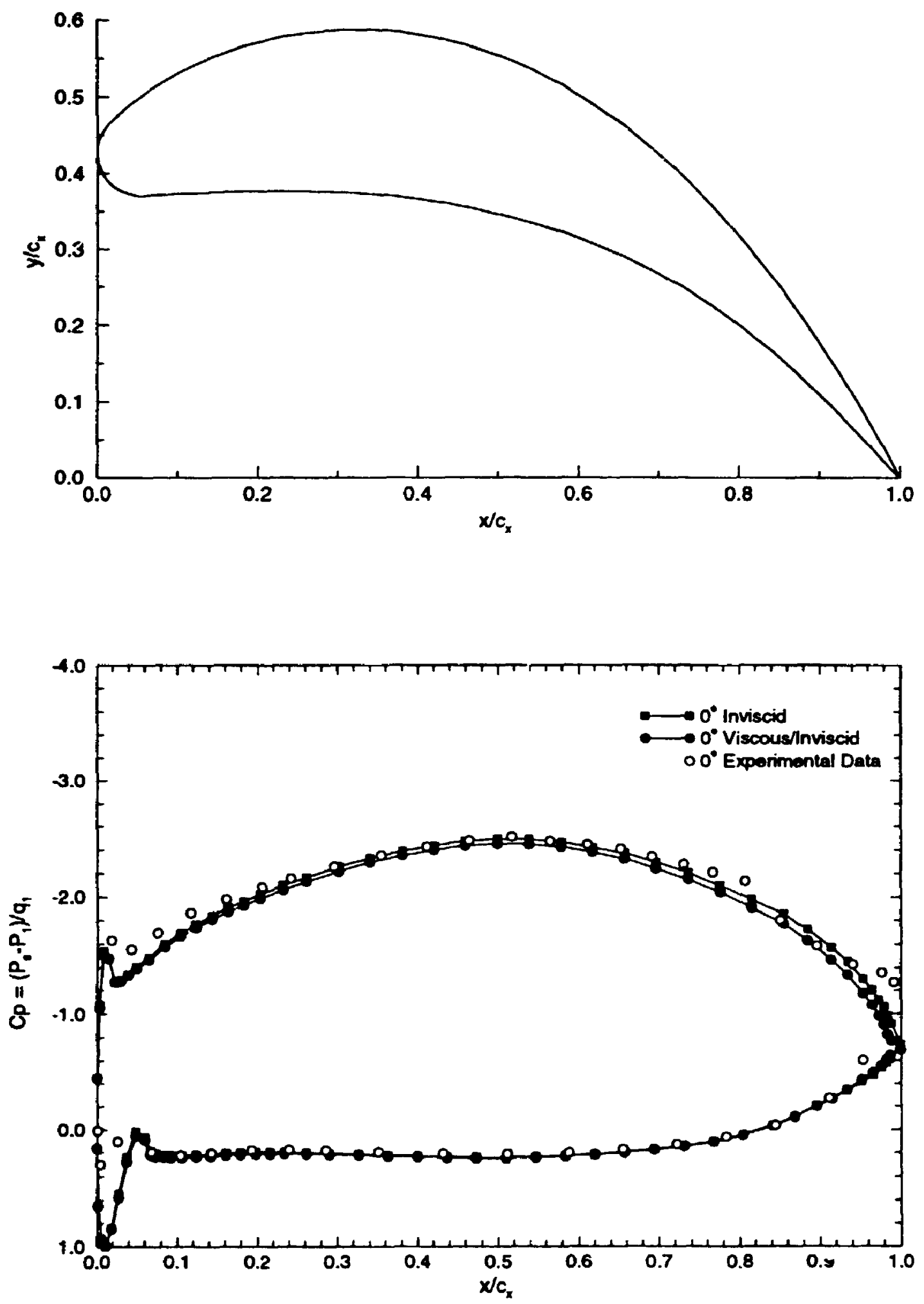

Figure 5.18: Pressure distributions and blade profile for the $\mathrm{CC} 3$ blade cascade; comparison with the experimental results, and the inviscid and viscous/inviscid predictions. 


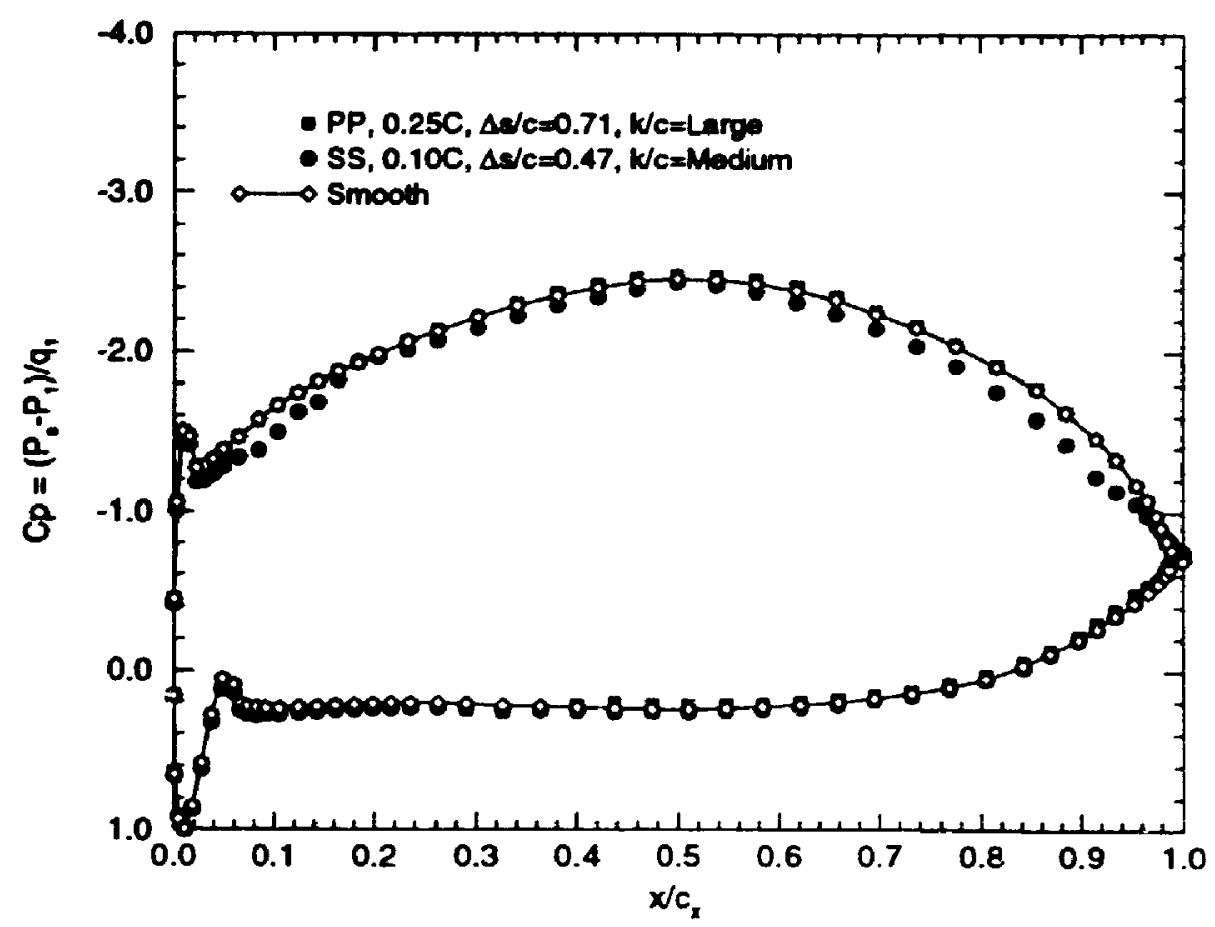

Figure 5.19: Predicted pressure distribution with and without roughness applied to the suction and pressure surface at design incidence.

\subsection{Rough-Surface Blades}

\subsection{Design Incidence}

Figure 5.19 shows the computationally predicted pressure distributions obtained at design incidence with and without roughness applied to the blade surface. The test cases shown are the computational reproduction of the test cases shown in Figure 5.3, that is, one without roughness (i.e. smooth), one with medium size roughness covering approximately $0.47 \Delta s / c$ of the suction surface with the roughness leading edge located at $0.10 x / c$, and one with large size roughness with the roughness leading edge located at $0.25 x / c$ and covering approximately $0.71 \Delta s / c$ of the pressure surface. As with the experimental results, the pressure distributions are not greatly affected by the surface roughness on the blades. The trends found in the experimental results are reproduced by the computational predictions although the predicted increase in suction on the suction 
surface due to roughness on the pressure surface is not as large as actually observed.

Consider the blades with roughness on the suction surface. A reduction in blade loading is apparent near the trailing edge. The boundary iayer analysis predicted laminar to turbulent transition at the leading edge of the roughness band. Comparing the boundary layer predictions for the smooth case and that with roughness on the suction surface showed a definite increase in the boundary layer thickness. For example, at 0.75 $x / c_{x}$ the boundary layer thickness, $\delta^{*}$, for the case with suction side roughness was approximately 3.5 times larger than that for the smooth case. The increase in boundary layer thickness reduces the curvature of the streamlines near the trailing edge and hence reduces the loading. This same trend was evident in the experimental measurements.

With roughness applied to the pressure surface, the computational analysis predicted essentially the same trends as did the experimental measurements. A slight increase in loading is observed near mid chord on the suction side although with not as great magnitude as the experimental measurements. Recall the explanation given in Section 5.1.2.2, that is, the increase in boundary layer thickness reduces the effective passage width which increases the passage velocity and the suction on the suction surface. Although this was somewhat evident in the computational predictions, a test case (not shown here) was performed with a smaller blade spacing to clarify this hypothesis. The pressure distributions were compared with a blade spacing three quarters of the original spacing. The blade loading on the suction surface increased by approximately $3 \%$ at mid chord with large roughness applied to the pressure side. This supports the hypothesis.

\subsection{Off-design Incidence}

Figure 5.20 shows a comparison of the pressure distributions at off-design incidence angles without roughness applied to the blades' surfaces. There is good agreement between the computational predictions and experimental measurements on the 


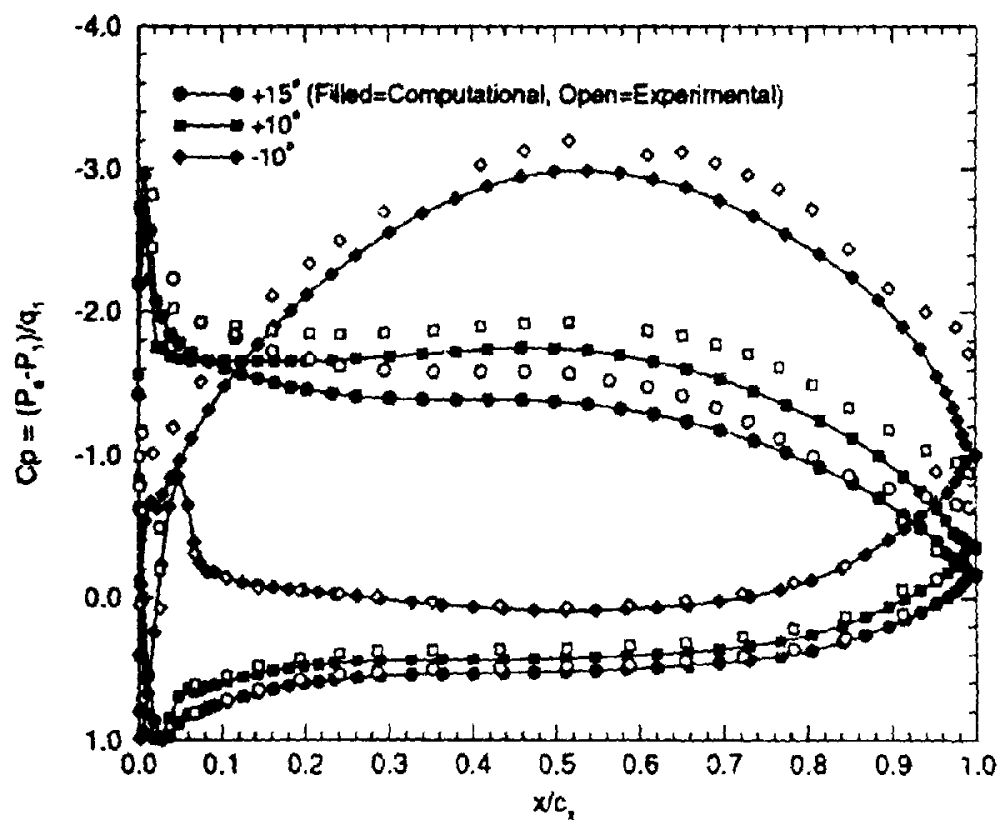

Figure 5.20: Predicted pressure distributions at off-design incidence angle; comparison with experimental data.

pressure surface at all aff-design incidence angles investigated. On the suction surface there is some discrepancy between the experimental measurements and the computational predictions. The discrepancy between the results was initially thought to have been caused by the modifications made to the blade trailing edge in the computational model. Further investigations involving choosing a different trailing edge point on the blade with the rounded trailing edge and then sharpening the blade to the new point did not appear to affect the suction surface pressure distribution significantly. The discrepancies may be due to sensitivity of the experimental measurements to the AVR at off-design incidence angles.

Figure 5.21 shows three predicted pressure distributions at $+10^{\circ}$ incidence. The test cases shown are the computational predictions corresponding to the test cases shown in Figure 5.5. As can be seen, roughness on the pressure surface does not greatly affect the pressure distribution. A decrease in the blade trailing edge loading is evident for the 


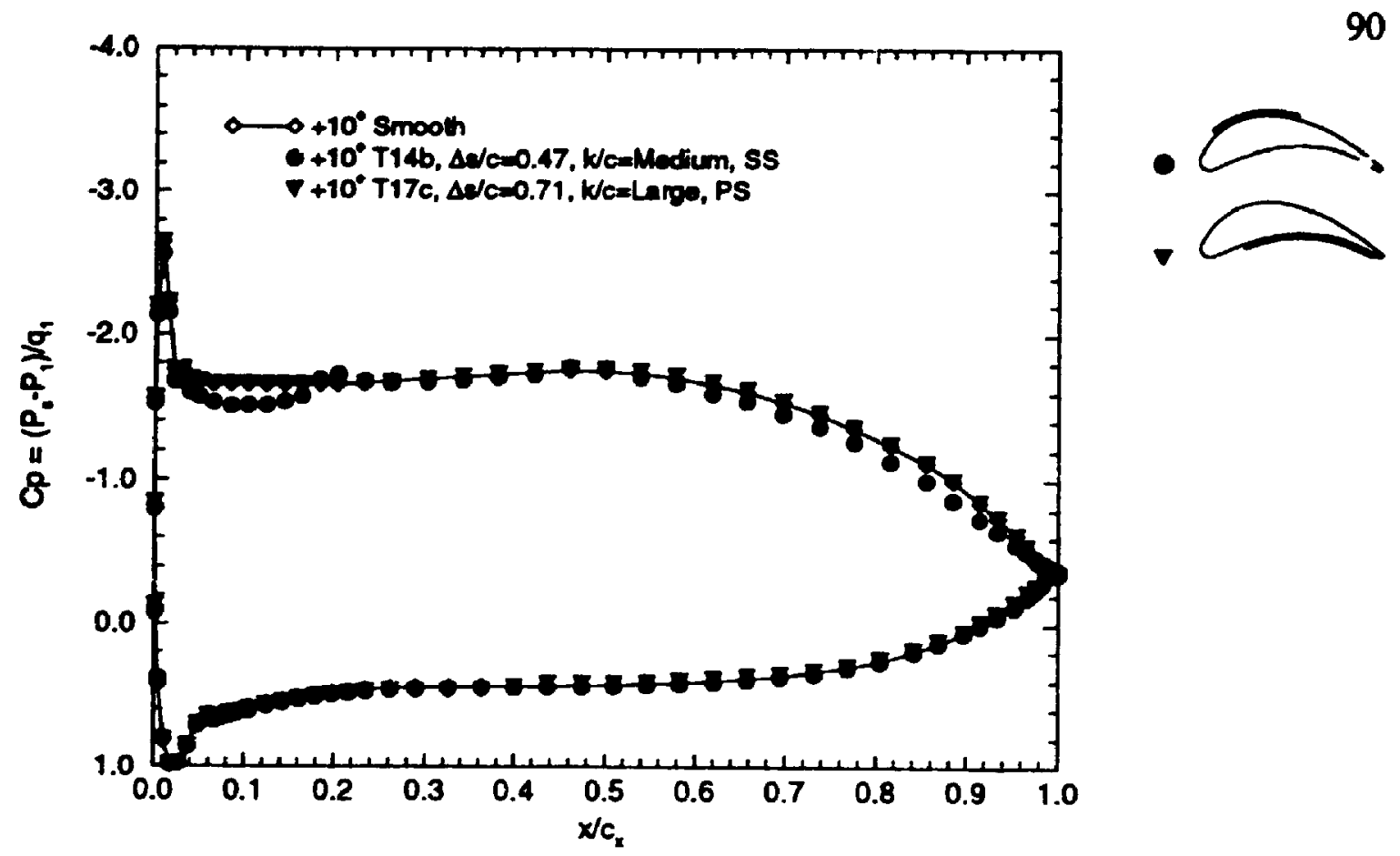

Figure 5.21: Predicted pressure distribution with and without roughness applied to the pressure and suction surfaces at $+10^{\circ}$ incidence .

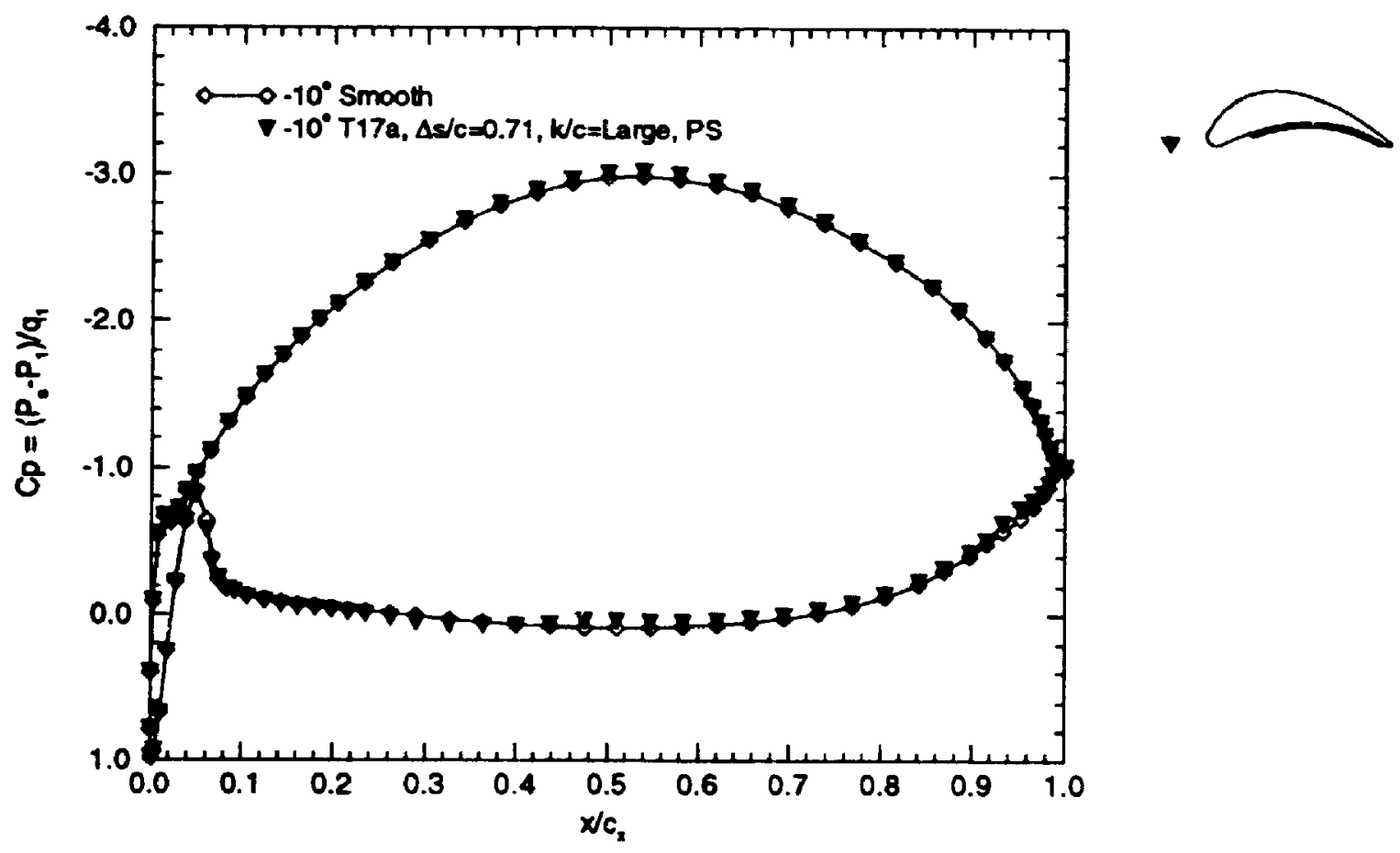

Figure 5.22: Predicted pressure distribution with and without roughness applied to the pressure surface at $-10^{\circ}$ incidence. 
case with roughness applied to the suction surface. These are the same trends evident ir. the experimental measurements.

Figure 5.22 shows two pressure distributions predicted at $-10^{\circ}$ incidence, one for a smooth case the other for large roughness covering approximately $0.71 \mathrm{\Delta s} / \mathrm{c}$ of the pressure surface. A definite increase in blade loading is evident on the suction surface, this was also the trend found in the experimental measurements shown in Figure 5.6.

\subsubsection{Pressure Loss and Flow Deviation}

\subsection{Design Incidence}

Figure 5.23 shows the variation of both measured and predicted loss coefficient $Y_{m}$, with non-dimensional roughness height for various test cases at design incidence. The same trends in loss were apparent in both the experimental measurements and computational predictions.

Figure 5.24 shows the variatio of both the measured and the predicted outlet-flow deviatior: angle, $\delta$, versus non-dimensional roughness height corresponding to the loss values shown in Figure 5.23. The computational predictions show the same trends observed in the experimental data, although magnitudes differ by about one half degree. Insignificant changes to the deviation angle (less than $0.1^{\circ}$ ) were correctly predicted by the computational method with roughness applied to the pressure surface. An increase in the deviation angle in the order of $1^{\circ}$ was predicted for roughness applied to the suction surface; this is slightly more than was observed experimentally. 


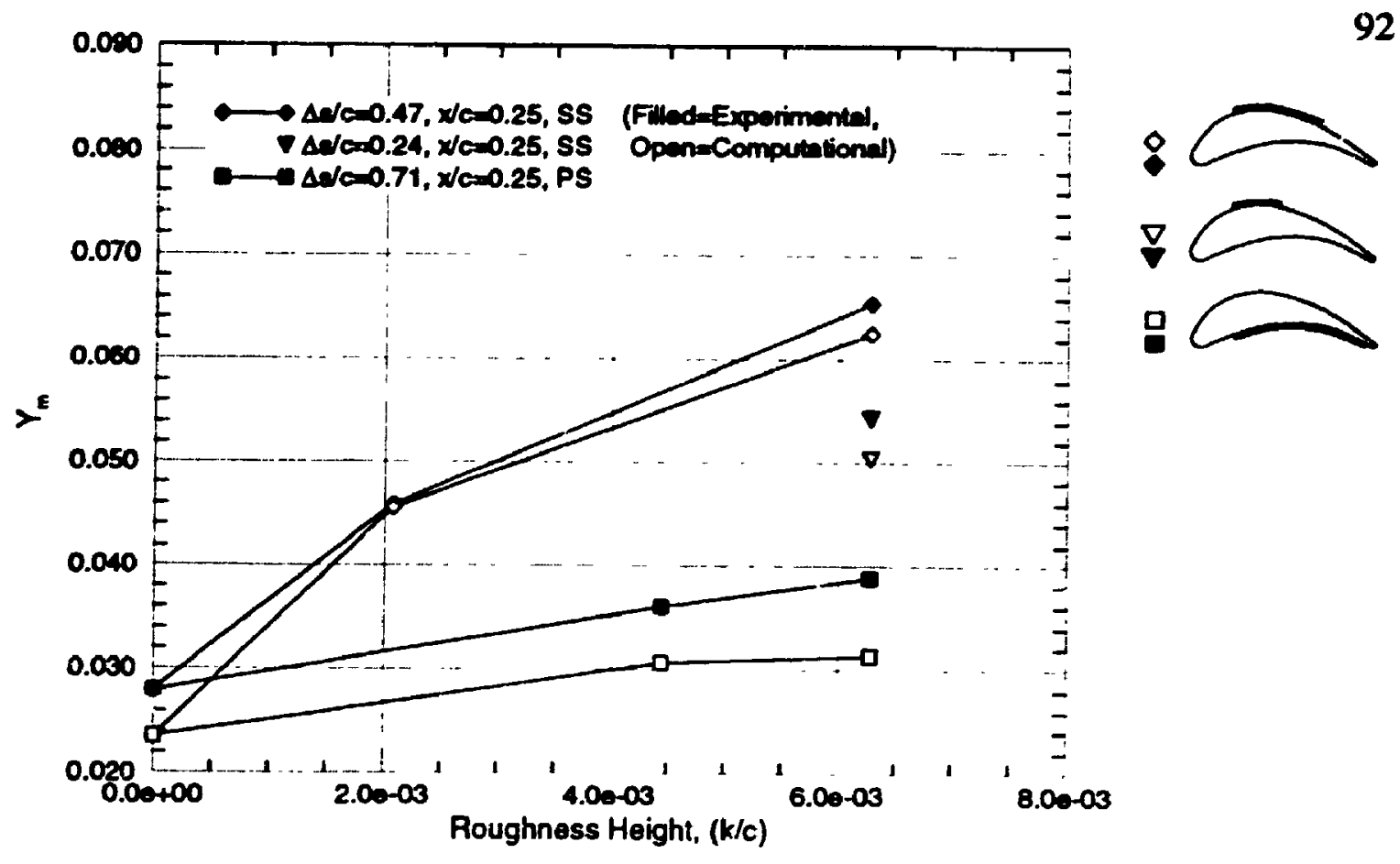

Figure 5.23: Predicted and measured total-pressure-loss coefficient versus roughness height, for design incidence.

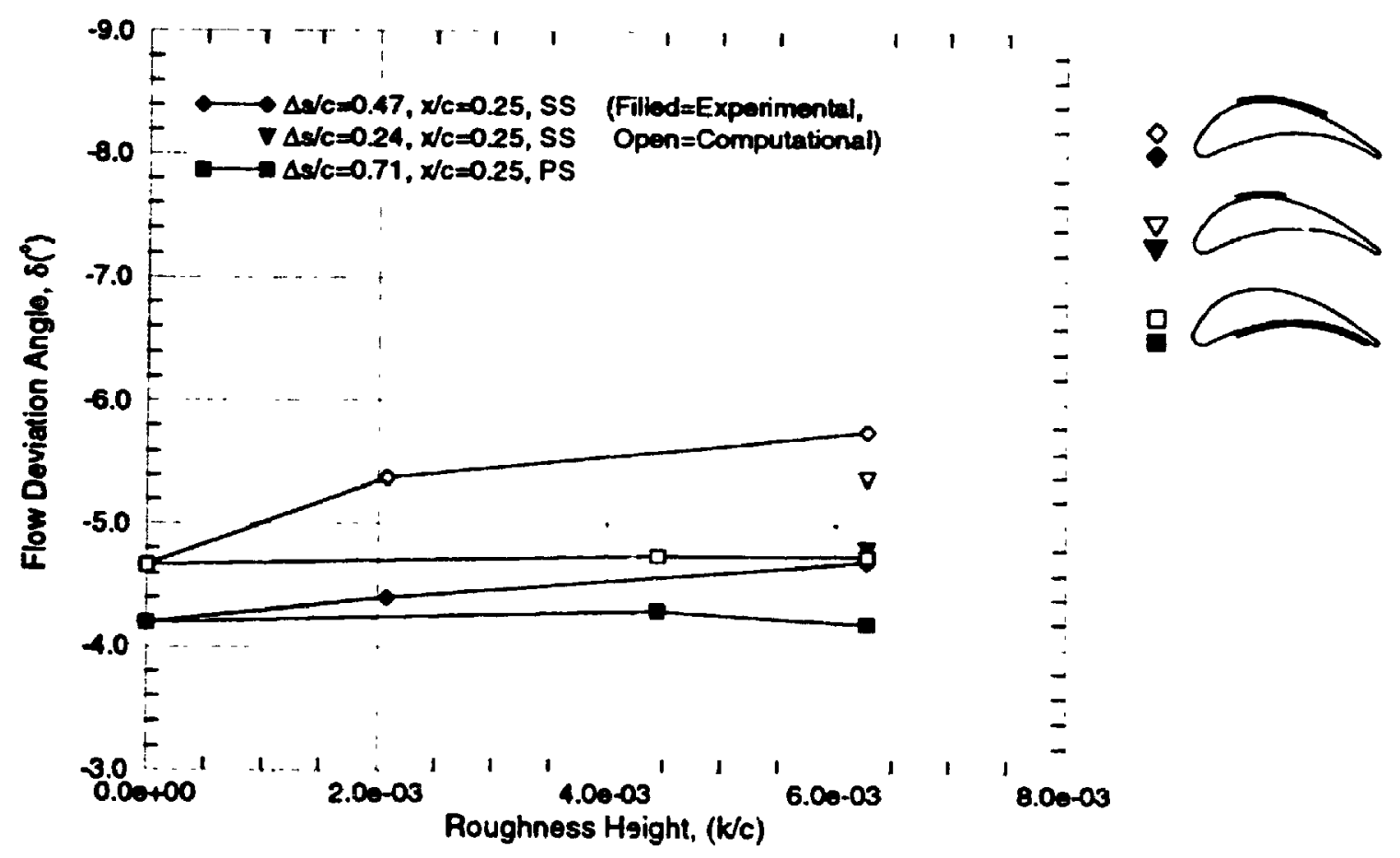

Figure 5.24: Predicted and measured flow deviation angle versus rcughness height, for design incidence. 


\subsection{Off-Design Incidence}

Figure 5.25 and 5.26 shows the variation of both the measured and the predicted loss coefficient for various roughness configurations at various angles of incidence. Also shown in both figures is the variation in the measured and the predicted loss coefficient for smooth blades at various angles of incidence. The computational method is limited to a positive incidence angle of $+10^{\circ}$. At incidence angles greater than $+10^{\circ}$, separated flow occurred and this cannot be modelled by the computational method in its current state of development. As can be seen, the trends in the loss coefficient for smooth blades is essentially the same for both the experimental measurements and computational predictions, although they differ slightly in magnitude. Included in Figure 5.25 are three loss curves illustrating the effect of roughness leading edge location on the suction surface. The roughness data illustrated in this figure are for the same roughness size (i.e. $k / c=0.00445$ ) and band width (i.e. $\Delta s / c=0.24$ ). It is clear from the experimental measurements that as the roughness band is positioned closer to the leading edge of the blade the losses increase. This is consistent with the results of Kind and Lawrysyn (1992) who predicted that roughness closer to the leauing edge of airfoils has a more detrimental effect. This trend is evident in the computational predictions for the roughness leading ediges positioned at $0.65 x / c$ and $0.25 x / c$. With the roughness leading edge located at $0.10 x i c$ a substantial increase in loss is apparent in the experimental data. The increase in loss is most likely due to the boundary layer trailing edge separation point moving upstream, thereby creating a larger separated region. Since separated flow is not modelled computationally, the computational method underpredicts the loss.

Figure 5.26 shows the variation in loss with medium and large size roughness applied to the pressure surface. The large roughness size causes only a slight increase in the losses relative to the medium size roughness for most incidence angles, a slight decrease in the experimentally measured loss was observed at $-10^{\circ}$. The decrease in loss at $-10^{\circ}$ was not observed in the computational predictions. A small increase in loss from 


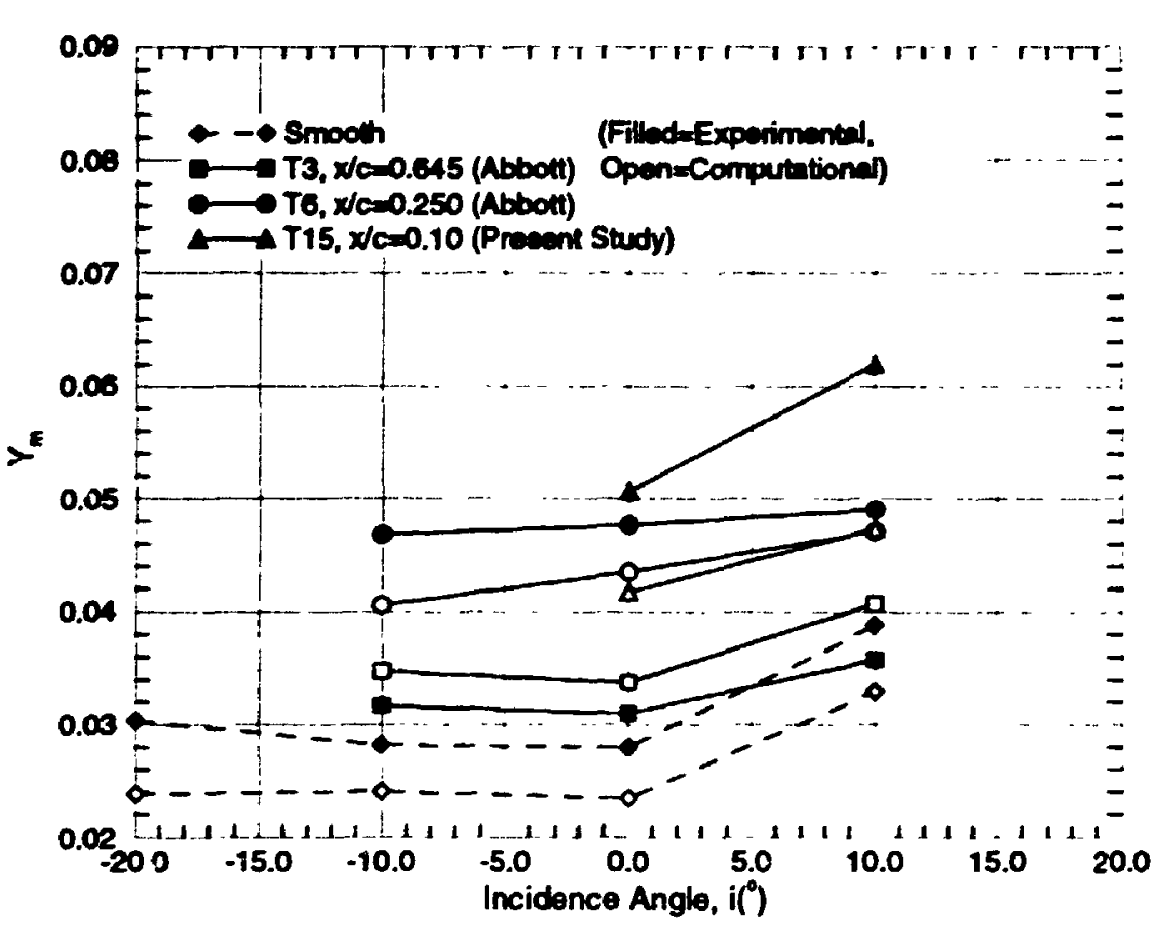

Figure 5.25: Predicted and measured total-pressure-loss coefficient versus incidence for various roughness locations on the suction surface (with $\mathrm{k} / \mathrm{c}=\mathbf{0 . 0 0 4 4 5}$, $\Delta s / c=0.24)$.

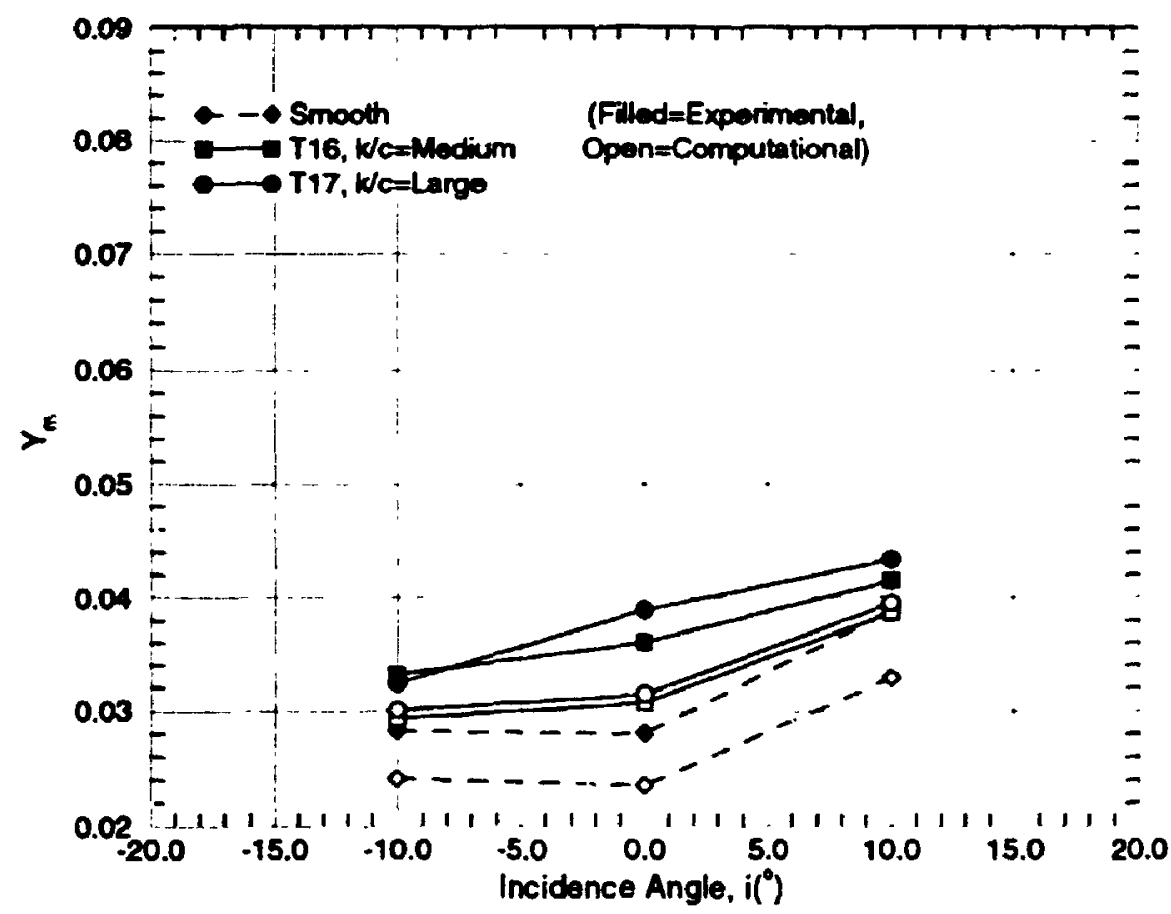

Figure 5.26: Predicted and measured total-pressure-loss coefficient versus incidence for medium and large size roughness on the pressure surface (with $x / c=0.25$ PS, $\Delta s / c=0.71$ ). 


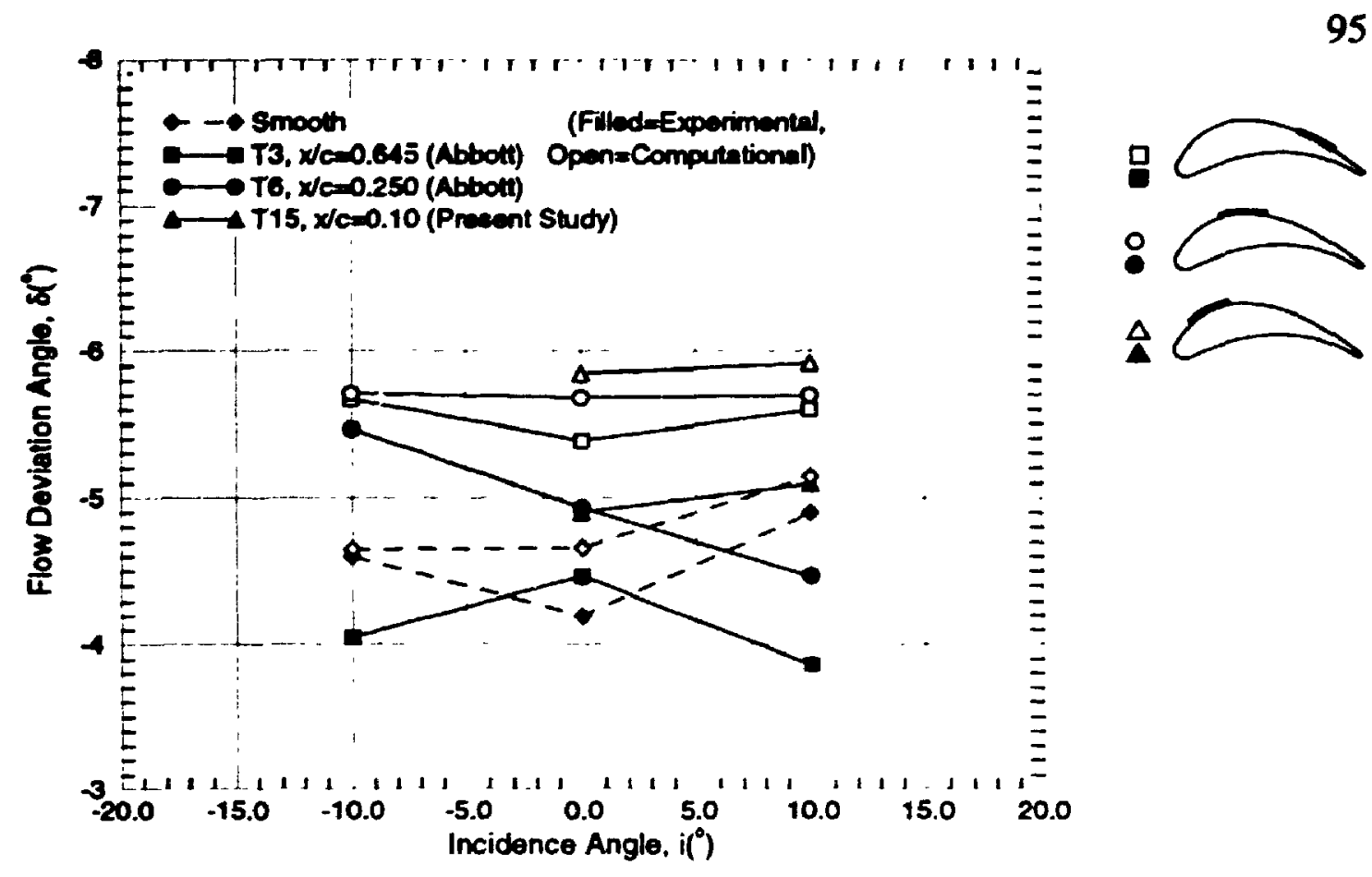

Figure 5.27: Predicted and measured flow deviation angle versus incidence for various roughness locations on the suction surface (with $\mathrm{k} / \mathrm{c}=0.00445, \Delta s \mathrm{c}=0.24$ SS).

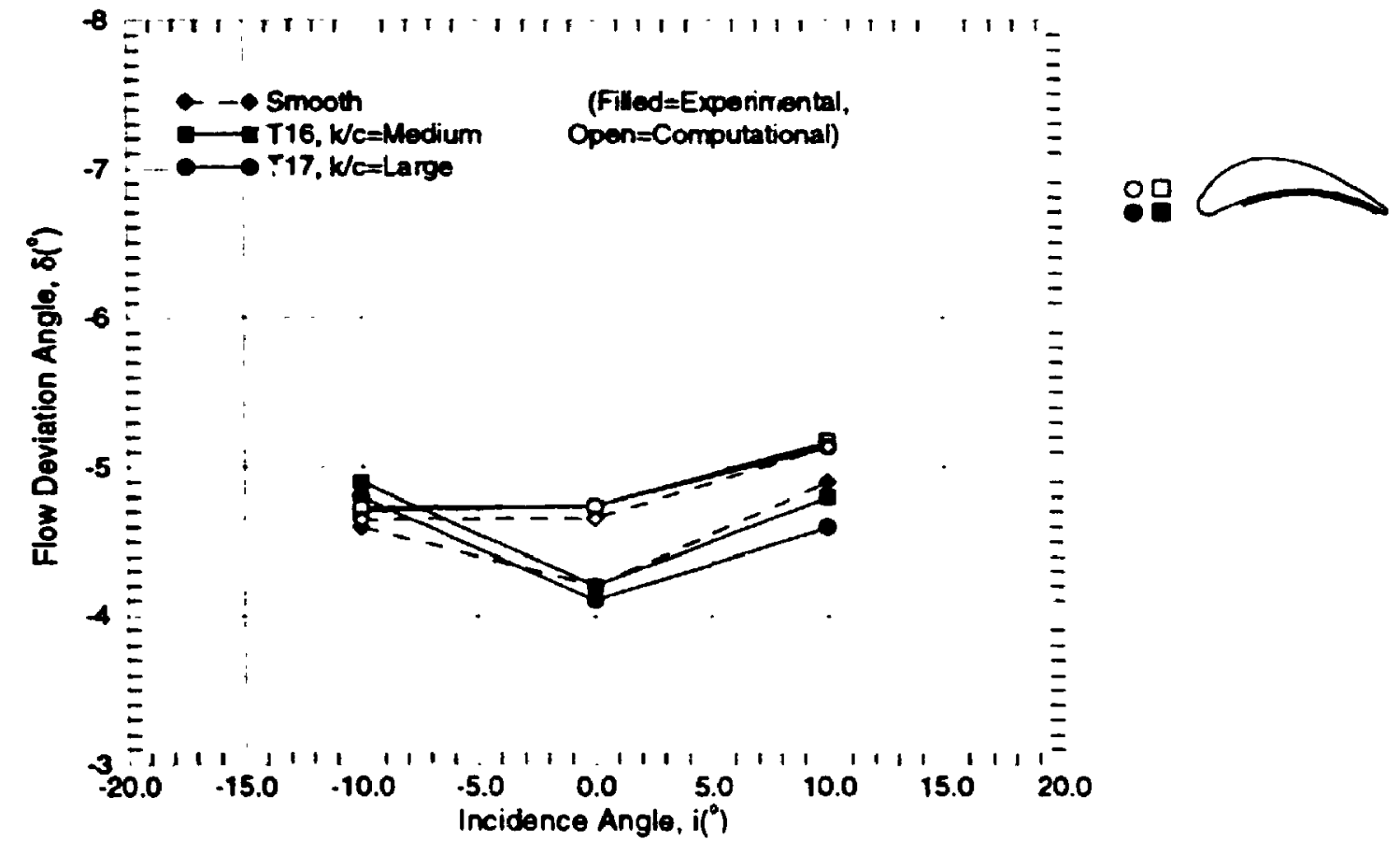

Figure 5.28: Predicted and measured flow deviation angle versus incidence for medium and large size roughness applied on the pressure surface (with $x / c=0.25$ PS, $\Delta \mathrm{s} / \mathrm{c}=0.71$ ). 


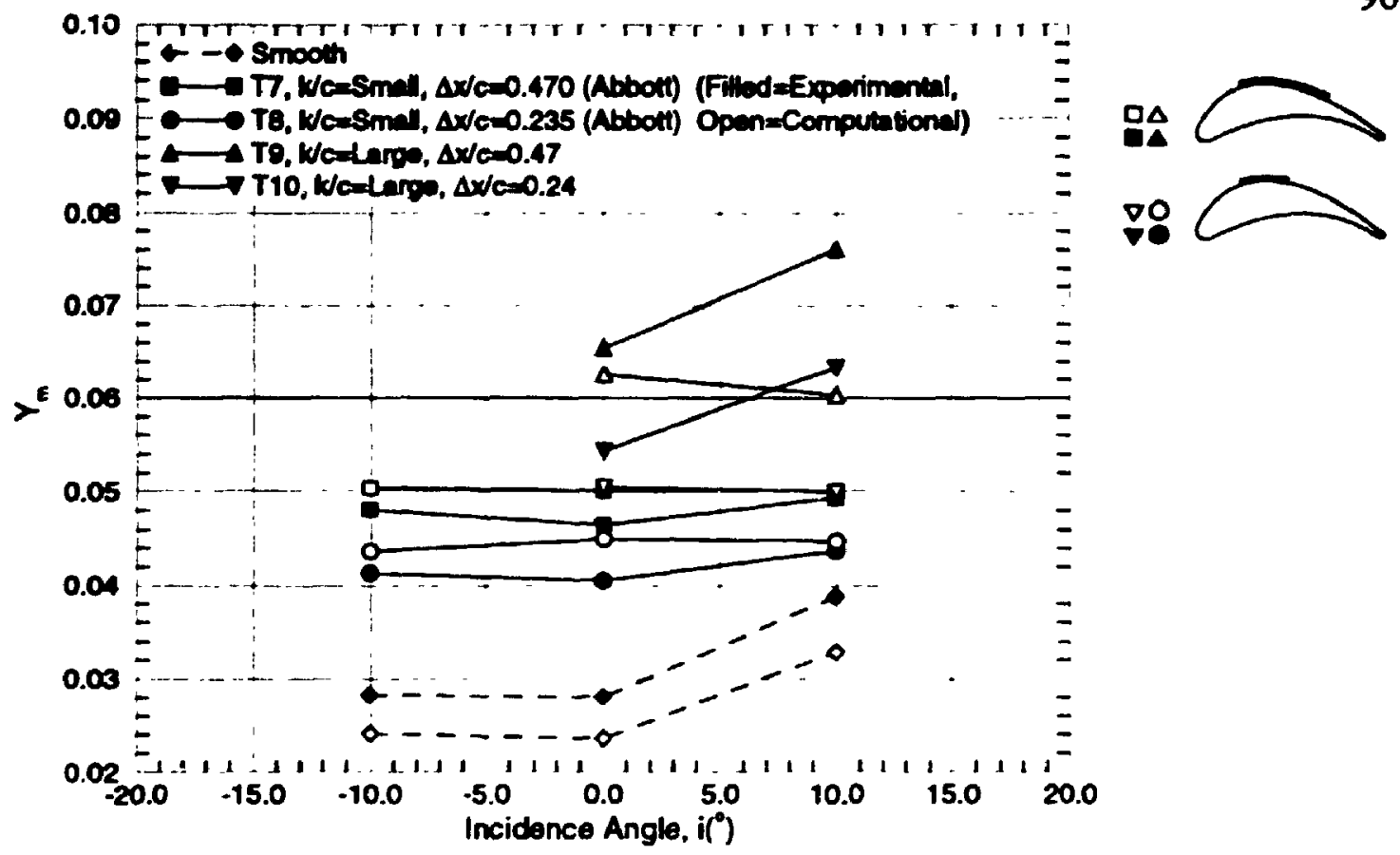

Figure 5.29: Predicted and measured total-pressure-loss coefficient versus incidence for large and small roughness sizes and for $0.24 \Delta \mathrm{s} / \mathrm{c}$ and $0.47 \Delta \mathrm{s} / \mathrm{c}$ roughness band widths $(\mathrm{LE}=0.25 \mathrm{x} / \mathrm{c}$ ).
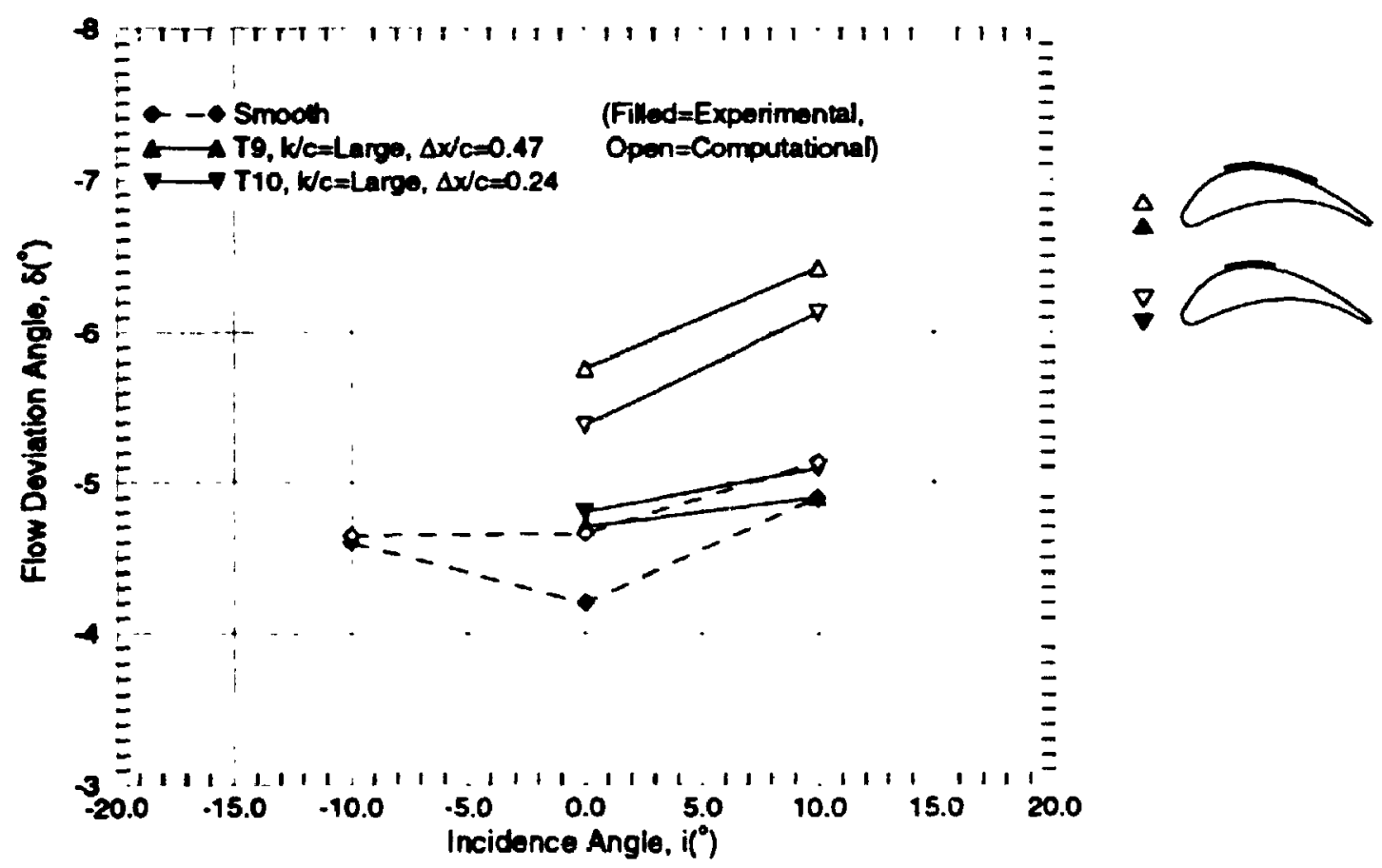

Figure 5.30: Predicted and measured flow deviation angle versus incidence for large and small roughness sizes and for $0.24 \Delta \mathrm{s} / \mathrm{c}$ and $0.47 \Delta \mathrm{s} / \mathrm{c}$ roughness band widths (LE $-0.25 \mathrm{x} / \mathrm{c}$ ). 
the smooth case was observed experimentally and computationally for both cases at all incidence angles.

The effects of roughness on the computationally and experimentally determined deviation angle are shown in Figure 5.27 and 5.28. Also included in the figures are the results for smooth blades. The trend in the experimental data for the smooth blades is captured by the computational method although the magnitude of the deviation angle predicted by the computations is slightly larger. Figure 5.27 shows the effect of roughness location on the deviation angle for the same cases shown in Figure 5.26. Consider the data determined by the computational predictions. An increase in the deviation angles is evident with roughness applied to the suction surface. The predicted deviation angles increase for all incidence angles shown as the roughness location is moved closer to the leading edge. The deviation angles for the case with the roughness leading edge located at $0.10 x / \mathrm{c}$ may not be representative of the actual deviation of the flow due to the large separation region near the trailing edge. The experimental deviation data does not show the same trends. An actual decrease in the deviation angle was recorded by Abbott (1993) for roughness located at $0.65 x / c$ and at $0.25 x / c$.

Figure 5.28 shows the effect of roughness on the deviation angle corresponding to the data shown in Fizure 5.26. As can be seen. the deviation angle is not greatly affected by the roughness on the pressure surface. A slight decrease in deviation from the smooth case is observed in the experimental measurements at design and $+10^{\circ}$ incidence angles. The computational predictions did not show a decrease in deviation at design and $+10^{\circ}$ incidence, although at $+10^{\circ}$ there is virtually no change in deviation from the smooth case.

Figure 5.29 shows the variation of both the measured and the predicted loss coefficients for large and small roughness sizes with the leading edges of the roughness bands located at $0.25 x / c$ and roughness band widths of $0.47 \Delta s / c$ and $0.24 \Delta s / c$ at varying angles of incidence. A uniform increase in loss is evident in the experimental 
measurements with both increasing roughness height and roughness band width. The computational predictions agree well with the experimental measurements for the small roughness samples at all angles of incidence and for large roughness samples at design incidence. At a $+10^{\circ}$ incidence angle the computational predictions of the total-pressure loss for the large roughness samples are substantially too low. As stated earlier, this is thought to be because the computational method does not consider separated flow, which actually occurs for these conditions.

The deviation angles corresponding to the pressure loss data for the large roughness height samples presented in Figure 5.29 are shown in Figure 5.30. A uniform increase in the deviation angles is evident in both the experimental measurements and computational predictions. 


\section{Chapter 6}

\section{CONCLUSIONS AND RECOMMENDATIONS}

\subsection{Conclusions}

The present research has investigated the effect of surface roughening on the aerodynamics of turbine blades. The following conclusions have been drawn from the observations made during this research:

1. The measured midspan static pressure distributions were not greatly affected by the presence of the roughness, although a small decrease in blade loading near the trailing edge was evident with roughness applied to the suction surface and a small increase in suction on the suction surface was evident with roughness applied to the pressure surface.

2. Laminar to turbulent transition occurred for all cases investigated near the leading edge of the roughness bands on both the suction and pressure surfaces.

3. Roughness produces the greatest effects when it occurs on the suction surface. The location of the roughness band on the suction surface was found to be important in determining the magnitude of the losse:s. With the roughness leading edge located at $0.10 x / c$ the increase in loss was the largest, whereas with the roughness leading edge located at $0.65 x / c$ only a small increase in loss was observed. 
4. Roughness applied to approximately $70 \%$ of the pressure surface resulted in only a small increase in losses. A slight decrease in the deviation angle was recorded for some of the cases investigated.

5. The total-pressure loss increases approximately linearly with increasing roughness height. A small increase in the deviation angle with roughness height was evident for roughness applied to the suction surface. Only very small changes in the deviation angle were recorded with roughness applied to the pressure surface.

6. The viscous/inviscid computational method showed great promise as a tool for investigating the aerodynamics of surface roughness on cascade blades. The computational method successfully predicted the trends observed in the measured results. The predicted pressure distributions with and without roughness applied to either the suction- or pressure- surfaces agreed well with the measured midspan pressure distributions. The total-pressure-loss coefficients and the flow deviation angles for the various roughness configurations investigated also agreed well with the data measured in the present study and the data of Abbott (1993).

\subsection{Recommendations for Further Study}

The experimental data obtained by Abbott (1993) in combination with the data obtained from the present study make up a fairly complete data base. No recommendations are made herein for further experimental work, instead the following outlines key areas of concern in the computational method.

1. It is evident from the experimental measurements that there is likely a small separation bubble at the leading edge of the blades for certain incidence angles. These separation bubbles could be modelled computationally with some 
modifications made to the viscous/inviscid methods.

2. The computational method loss and flow-tuming predictions were limited to flows where the separated region at the trailing edge was small. The applicability of the computational method would be improved with the inclusion of a separated flow model for cascades.

3. The computational method could be used to expand from the experimental data set and investigate the effects of other parameters such as blade geomerry, roughness height and spacing, and Reynolds number. 


\section{REFERENCES}

Abbott, M. W. P., 1993, "The Aerodynamic Effect of Roughness on the Performance of a Turbine Cascade," M.Eng. Thesis, Department of Mechanical and Aerospace Engineering, Carleton University, Ottawa, Canada.

Baldwin, B. S., Lomax, H., 1978, "Thin-Layer Approximation and Algebraic Model for Separated Turbulent Flows," AIAA Paper 78-257.

Bammert, K., and Milsch, R., 1972, "Boundary Layers on Rough Compressor Blades," ASME Paper 72-GT-48.

Bammert. K., Sandstede, H., 1980, "Measurements of the Boundary Layer Development Along a Turbine Blade with Rough Surfaces," ASME Journal of Engineering for Power, Vol. 102, pp 978-983.

Benner, M. W., Sjolander S. A., and Moustapha, S. H., 1995, "Influence of Leading-Edge Geometry on Profile Losses in Turbines at Off-Design Incidence: Experimental Results and an Improved Correlation," Submitted for. ASME-IGTI Tubo Expo, Houston.

Bittle, S. A., 1985, "Aı Analysis Method for the Viscous/Inviscid Flow Around Airfoils With Extensive Tro 'ng Edge Separation Including Surface Roughness Effects," M.Eng. Thesis, Department of Mechanical and Aerospace Engineering, Carleton University, Ottawa, Canada.

Boyle, R. J., 1993, 'Prediction of Surface Roughness and Incidence Effects on Turbine Performance," ASME Paper 93-GT-280 (also available in: ASME Journal of Turbomachinery, Vol. 116, pp. 745-751).

Boynton, J. L., Tabibzadeh, R., and Hudson, S. T., 1992, "Investigation of Rotor Blade Roughness Effects on Turbine Performance," ASME Paper 92-GT-297 (also available in: ASME Journal of Turbomachinery, Vol. 115, pp. 614-620).

Cebeci, T., and Chang, K. C., 1978, "Calculation of Incompressible RoughWall Boundary Layer Flows," AIAA Journal, Vol. 16, No.7. pp 730-735. 
Cue, R. W., and Muir, D. E., 1991, 'Engine Performance Monitoring and Troubleshooting Techniques for the CF-18 Aircraft," ASME Journal of Engineering for Gas Turbines and Power, Vol. 113, pp. 11-19.

Dvorak, F. A., 1969, 'Calculation of Turbulent Boundary Layers on Rough Surfaces in Pressure Gradient," AIAA Journal, Vol. 7, No. 9, pp 1752-59.

Goobie, S. M., 1989, "A Test Section for Investigating the Off-Design Performance of Planar Cascades," M.Eng. Thesis, Department of Mechanical and Aerospace Engineering, Carleton University, Otawa, Canada.

Gostellow, J. P., 1984, Cascade Aerodynamics, Pergamon Press Ltd., Toronto.

Head, M. R., 1953, "Entrainment in the Tlurbulent Boundary Layer," A.R.C. R\&M 3152.

Head, M. R., and Patel, V.C.. 1968, "Improved Entrainment Method for Calculating Turbulent Boundary Layer Development," R\&M 3643.

Issacs, D. P., 1994, "The Aerodynamics of Turbine Blades with Trailing Edge Damage," M.Eng. Thesis, Department of Mechanical and Aerospace Engineering. Carleton University. Ottawa, Canada.

Kacker, S. C., and Okapuu, U.. 1982, "A Mean Line Prediction Method for Axial Flow Turbine Efficiency." ASME Journal of Engineering for Power. Vol. 104. pp. 111-119.

Kind. R. J., 1973, "An Improved Surface-Vorticity Method of Solution for Potential Flow Over Arbitrary Airfoils." Internal Report. Carleton University. Ottawa. Canada.

Kind. R. J., 1992. Addendum to. "An Improved Surface-Vorticity Method of Solution for Potential Flow Over Arbitrary Airfoils," Internal Report, Carleton University, Ottawa, Canada.

Kind, R. J.. and Lawrysyn, M. A.. 1992, 'Performance Degradation Due to Hoar Frost on Lifting Surfaces," Canadian Astronautics and Space Journal, Vol. 38. No. 2. June 1992, pp. 62-70.

Lewis, W. E., 1966, "Fixed-Direction Probes for Aerodynamic Measurements." Proceedings of the Institution of Mechanical Engineers. Vol. 180. Pt. 3J. 1965-66. pp. 141-152. 
Rodger, P., 1992, 'Establishing Two-Dimensional Flow in a Large-Scale Planar Turbine Cascade," M.Eng. Thesis, Department of Mechanical and Aerospace Engineering, Carleton University, Ottawa, Canada.

Sjolander, S. A., Issacs, D. P., and Klein, W. A., 1993, "Aerodynamics Turbine Blades with Trailing-Edge Damage: Measurements and Computations," Proceedings, XI International Symposium on Air Breathing Engines, Vol. II, pp. 13271334.

Speidel, L., 1954, "Emfly der Oberflăchenraichigkeit auf die Strömungsverluste in ebenen Schaufelgittern," Forschung auf dem Gabiete des Ingenieurwesens, Band 20, Nr. 5, 1954, pp. 129-140.

Squire, H. B., and Young, A. D., 1938, "The Calculation of the Profile Drag of Aerofoils," ARC R\&M 1838.

Suder, K. L., Ch:ma, R. V., Strazisar, A. J., and Roberts, W. B., 1994, "The Effect of Adding Roughness and Thickness to a Transonic Axial Compressor Rotor," ASME Paper 94-GT-339.

Thwaites, B., 1949, "An Approximate Calculation for the Laminar Boundary Layer," Aeronautical Quarterly, I, 245.

Tremblay, B., 1989, "Measurement and Correlation of Off-Design Performance of Axial-Flow Turbines," M.Eng. Thesis, Department of Mechanical and Aerospace Engineering, Carleton University, Ottawa, Canada.

Wazzan, A. R., Gazley, Jr. C., and Smith, A.M.O., 1981, "H-R Method for Predicting Transition," AIAA Vol. 19, No. 6, pp. 810-812.

Whitehouse, D. R., 1993, "The Effect of Axial Velocity Ratio, Turbulence Intensity, Incidence and Leading Edge Geometry on the Off-Design Performance of a Turbine Blade Cascade," M.Eng. Thesis, Department of Mechanical and Aerospace Engineering, Carleton University, Ottawa, Canada.

Yaras, M. I., 1987, "Flow in the Tip Gap of a Rectangular Turbine Cascade," M.Eng. Thesis, Department of Mechanical and Aerospace Engineerivg, Carleton University, Ottawa, Canada. 


\section{Appendix A}

\section{Blade and Cascade Geometry}

This appendix tabulates the geometric and aerodynamic parameters of the cascade and the coordinates used to describe the profile.

Table A.1: Summary of geometric and aerodynamic parameters of the cascade blades.

\begin{tabular}{|c|c|}
\hline Inlet flow angle, $\alpha_{1}$ & $28.35^{\circ}$ \\
\hline Exit flow angle, $\alpha_{2}$ & $57.65^{\circ}$ \\
\hline Inlet metal angle, 6 , & $25.35^{\circ}$ \\
\hline Exit metal angle, $6_{2}$ & $57.51^{\circ}$ \\
\hline Metal turming, $\Delta 6$ & $83.0^{\circ}$ \\
\hline Design incidence, $i_{\text {. }}$ & $3.0^{\circ}$ \\
\hline Design deviation, $\delta_{\text {d. }}$ & $-0.145^{\circ}$ \\
\hline Stagger, $\gamma$ & $21.6^{\circ}$ \\
\hline Camber angle, $\theta$ & $82.86^{\circ}$ \\
\hline Chord length, $c$ & $162.3 \mathrm{~mm}$ \\
\hline Axial chord length, $c_{x}$ & $149.4 \mathrm{~mm}$ \\
\hline Pitch (spacing), $\mathbf{S}$ & $110.71 \mathrm{~mm}$ \\
\hline Blade span, $\mathbf{h}$ & $200 \mathrm{~mm}$ \\
\hline Pitch to chord ratio, S/c & 0.68 \\
\hline Aspect ratio, $\mathbf{b} / \mathbf{c}$ & 1.23 \\
\hline Leading edge diameter to chord ratio, $\mathbf{d} / \mathbf{c}$ & 0.1029 \\
\hline Leading edge diameter to pitch ratio, dS & 0.151 \\
\hline Leading edge wedge angle. We & $43.0^{\circ}$ \\
\hline Trailing edge thickness to chord ratio. $/ c$ & 0.0259 \\
\hline Maximum thickness to chord, $t_{\operatorname{man}}$ /c & $19.6 \%$ \\
\hline Throat opening, $\sigma$ & $63.6 \mathrm{~mm}$ \\
\hline
\end{tabular}


Table A.2: Blade profile coordinates.

\begin{tabular}{|c|c|c|c|c|c|c|c|}
\hline \multicolumn{4}{|c|}{ Suction Surface } & \multicolumn{4}{|c|}{ Preasure Surfece } \\
\hline $\boldsymbol{x} / \mathbf{c}$ & $y / c$ & ע/c & $y / c$ & $\boldsymbol{J} / \mathrm{c}$ & $y / z$ & $\mathbf{x} / \mathrm{c}$ & $y / c$ \\
\hline 0.0050 & 0.3578 & 0.4209 & 0.5099 & 0.0050 & 0.3578 & 0.4337 & 0.3133 \\
\hline 0.0015 & 0.3674 & 0.4571 & 0.4977 & 0.0102 & 0.3490 & 0.4674 & 0.3052 \\
\hline 0.0001 & 0.3776 & 0.4933 & 0.4822 & 0.0171 & 0.3415 & 0.5010 & 0.2955 \\
\hline 0.0006 & 0.3878 & 0.5294 & 0.4631 & 0.0254 & 0.3355 & 0.5347 & 0.2840 \\
\hline 0.0032 & 0.3977 & 0.5656 & 0.4402 & 0.0347 & 0.3312 & 0.5684 & 0.2707 \\
\hline 0.0077 & 0.4069 & 0.6018 & 0.4134 & 0.0447 & 0.3288 & 0.6020 & 0.2555 \\
\hline 0.0139 & 0.4150 & 0.6380 & 0.3825 & 0.0549 & 0.3285 & 0.6357 & 0.2381 \\
\hline 0.0162 & 0.4174 & 0.6742 & 0.3473 & 0.0570 & 0.3286 & 0.6694 & 0.2184 \\
\hline 0.0207 & 0.4220 & 0.7103 & 0.3078 & 0.0612 & 0.3289 & 0.7031 & 0.1961 \\
\hline 0.0275 & 0.4286 & 0.7165 & 0.2637 & 0.0675 & 0.3293 & 0.7367 & 0.1709 \\
\hline 0.0365 & 0.4367 & $0.782 i$ & 0.2153 & 00759 & 0.3298 & 0.7704 & 0.1424 \\
\hline 0.0456 & 0.4442 & 0.8098 & 0.1764 & 0.0844 & 0.3303 & 0.7957 & 0.1186 \\
\hline 0.0591 & 0.4544 & 0.8370 & 0.1356 & 0.0970 & 0.3310 & 0.8209 & 0.0922 \\
\hline 0.0772 & 0.4665 & 0.8550 & 0.1076 & 0.1138 & 0.3319 & 0.8377 & 0.0731 \\
\hline 0.0953 & 0.4771 & 0.8731 & 0.0791 & 0.1306 & 0.3326 & 0.8546 & 0.0525 \\
\hline 0.1134 & 0.4863 & 0.8912 & 0.0502 & 0.1475 & 0.3332 & 0.8714 & 0.0303 \\
\hline 0.1315 & 0.4945 & 0.9048 & 0.0285 & 0.1643 & 0.3337 & 0.8840 & 0.0125 \\
\hline 0.1496 & 0.5016 & 0.9116 & 0.0177 & 0.1812 & 0.3340 & 0.8904 & 0.0032 \\
\hline 0.1677 & 0.5077 & 0.9161 & 0.0104 & 0.1980 & 0.3342 & 0.8946 & -0.0031 \\
\hline 0.1858 & 0.5130 & 0.9184 & 0.0068 & 0.2148 & 0.3343 & 0.8967 & -0.0070 \\
\hline 0.2129 & 0.5192 & 0.9197 & 0.0038 & 0.2401 & 0.3340 & n. $8 \mathbf{E}^{\circ} . \dot{0}$ & -0.0095 \\
\hline 0.2400 & 0.5237 & 0.9203 & 0.0005 & 0.2653 & 0.3334 & 0.9016 & -0.0114 \\
\hline 0.2762 & 0.5270 & 0.9200 & -0.0028 & 0.2990 & 0.3317 & 0.9047 & -0.0126 \\
\hline 0.3124 & 0.5273 & 0.9188 & $\Omega 0060$ & C. 3327 & 0.3290 & 0.9080 & -0.0128 \\
\hline 0.3486 & 0.5245 & 0.9169 & -0.0087 & 0.3663 & $0.325 !$ & 0.9113 & -0.0123 \\
\hline 0.3847 & 0.5188 & 0.9144 & -0.0108 & 0.4000 & 0.3199 & 0.9144 & -0.0108 \\
\hline
\end{tabular}




\section{Appendix B}

\section{Static Pressure Blade Geometry}

This appendix lists the blade surface static tap locations and orientation relative to the coordinate system shown in Figure B.1.

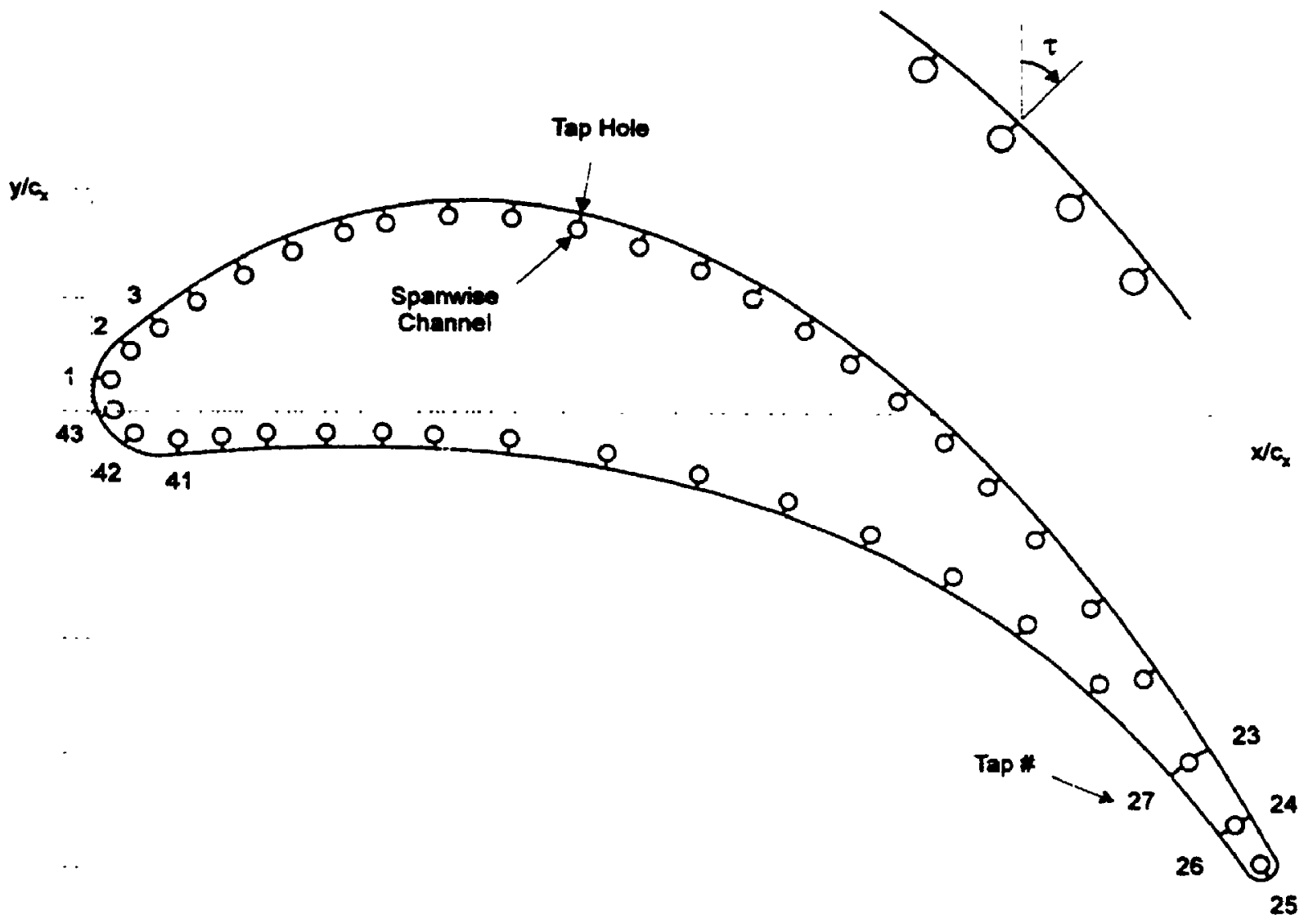

Figure B.1: Static tap location and orientation nomenclature for the instrumented blade. 
Table B.1: Static pressure tap coordinates and orientation.

\begin{tabular}{|c|c|c|c|c|c|c|c|}
\hline \multicolumn{4}{|c|}{ Suction Surfince } & \multicolumn{4}{|c|}{ Pressure Surface } \\
\hline Tap \# & $x / c_{x}$ & $y / c_{x}$ & +9 & Tap * & $x / c$ & $y / c_{2}$ & $\tau(9)$ \\
\hline 1 & 0.0014 & 0.0281 & -77.185 & 43 & 0.0048 & -0.0068 & -113.896 \\
\hline 2 & 0.0182 & 0.0573 & -45.898 & 42 & 0.0264 & -0.0317 & -148.219 \\
\hline 3 & 0.0431 & 0.0805 & -39.928 & 41 & 0.0682 & -0.0394 & 176.210 \\
\hline 4 & 0.0755 & 0.1045 & -33.511 & 40 & 0.1049 & -0.0373 & 176.978 \\
\hline 5 & 0.1170 & 0.1284 & -26.574 & 39 & 0.1430 & -0.0355 & 177.775 \\
\hline 6 & 0.1614 & 0.1476 & -20.210 & 38 & 0.1928 & -0.0341 & 178.941 \\
\hline 7 & 0.2061 & 0.1615 & -14.356 & 37 & 0.2405 & -0.0338 & -179.689 \\
\hline 8 & 0.2423 & 0.1693 & .9 .833 & 36 & 0.2860 & -0.0347 & -178.078 \\
\hline 9 & 0.2961 & 0.1754 & -3.344 & 35 & 0.3521 & -0.0387 & -175.081 \\
\hline 10 & 0.3547 & 0.1750 & 4.202 & 34 & 0.4224 & -0.0489 & -170.208 \\
\hline 11 & 0.4109 & 0.1681 & 10.055 & 33 & 0.5122 & -0.0665 & -164.880 \\
\hline 12 & 0.4638 & 0.1551 & 16.992 & 32 & 0.5880 & -0.0909 & -159.320 \\
\hline 13 & 0.5172 & 0.1355 & 23.365 & 31 & 0.6554 & -0.1199 & -154.071 \\
\hline 14 & 0.5649 & 0.1120 & 28.938 & 30 & 0.7223 & -0.1565 & -148.543 \\
\hline 15 & 0.6113 & 0.0835 & 34.090 & 29 & 0.7835 & -0.1980 & -143.100 \\
\hline 16 & 0.6523 & 0.0536 & 38.357 & 28 & 0.8449 & -0.2492 & -137.264 \\
\hline 17 & 0.6917 & 0.0200 & 42.216 & 27 & 0.9123 & -0.3199 & -129.894 \\
\hline 18 & 0.7309 & -0.0179 & 45.779 & 26 & 0.9533 & -0.3728 & -125.503 \\
\hline 19 & 0.7677 & -0.0578 & 48.821 & 25 & 0.9927 & -0.3968 & 146.633 \\
\hline 20 & 0.8073 & -0.1055 & 51.720 & & & & \\
\hline 21 & 0.8509 & -0.1664 & 54.486 & & & & \\
\hline 22 & 0.8971 & -0.2307 & 56.470 & & & & \\
\hline 23 & 0.9410 & $-0,2987$ & 57.663 & & & & \\
\hline 24 & 0.9774 & -0.3566 & 58.007 & & & & \\
\hline
\end{tabular}




\section{Appendix C}

\section{Calibration Data}

This appendix presents the calibration coefficients and resulting curves for the three-hole probes and the calibration curves for the pressure transducers.

The inlet and outlet three-hole probes were previously calibrated by Abbott (1993) in May 1993. They were recalibrated by the author in May 1994 and August 1994. The calibration data are used to determine the static pressure, dynamic pressure, total pressure and flow direction. The probe was calibrated over a range of yaw angles from $-10^{\circ}$ to $+10^{\circ}$. The measured pressures from the calibration are used to form the following coefficients according to the method of Lewis (1966),

$$
\begin{aligned}
& K_{1}=\frac{\left(P_{1}-P_{s}\right)}{q} \\
& K_{12}=\frac{\left(P_{1}-P_{2}\right)}{q} \\
& K_{13}=\frac{\left(P_{1}-P_{3}\right)}{q} \\
& K_{23}=\frac{\left(P_{2}-P_{3}\right)}{q} \\
& K_{\phi 2}=\frac{\left(P_{1}-P_{2}\right)}{\left(P_{1}-P_{3}\right)} \\
& K_{\phi 3}=\frac{\left(P_{1}-P_{3}\right)}{\left(P_{1}-P_{2}\right)}
\end{aligned}
$$

where: $P_{s}=$ Static pressure at probe $q$ - Dynamic pressure at probe 
The pressure indices correspond to the port numbering shown in Figure C.1. The coefficients are graphically presented in Figures C.2 to C.7 for the outlet probe. Similar results (not shown here) were obtained for the inlet probe. As can be seen, the calibration of the probe has been very stable.

(+) Pitch Angle

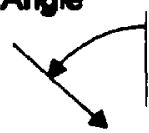

(+) Yaw Angle<smiles>CCCCCCCC</smiles>

Anglo

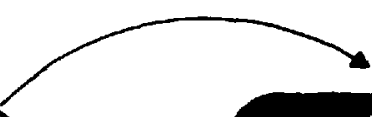

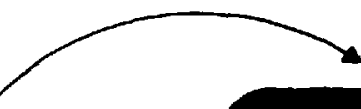




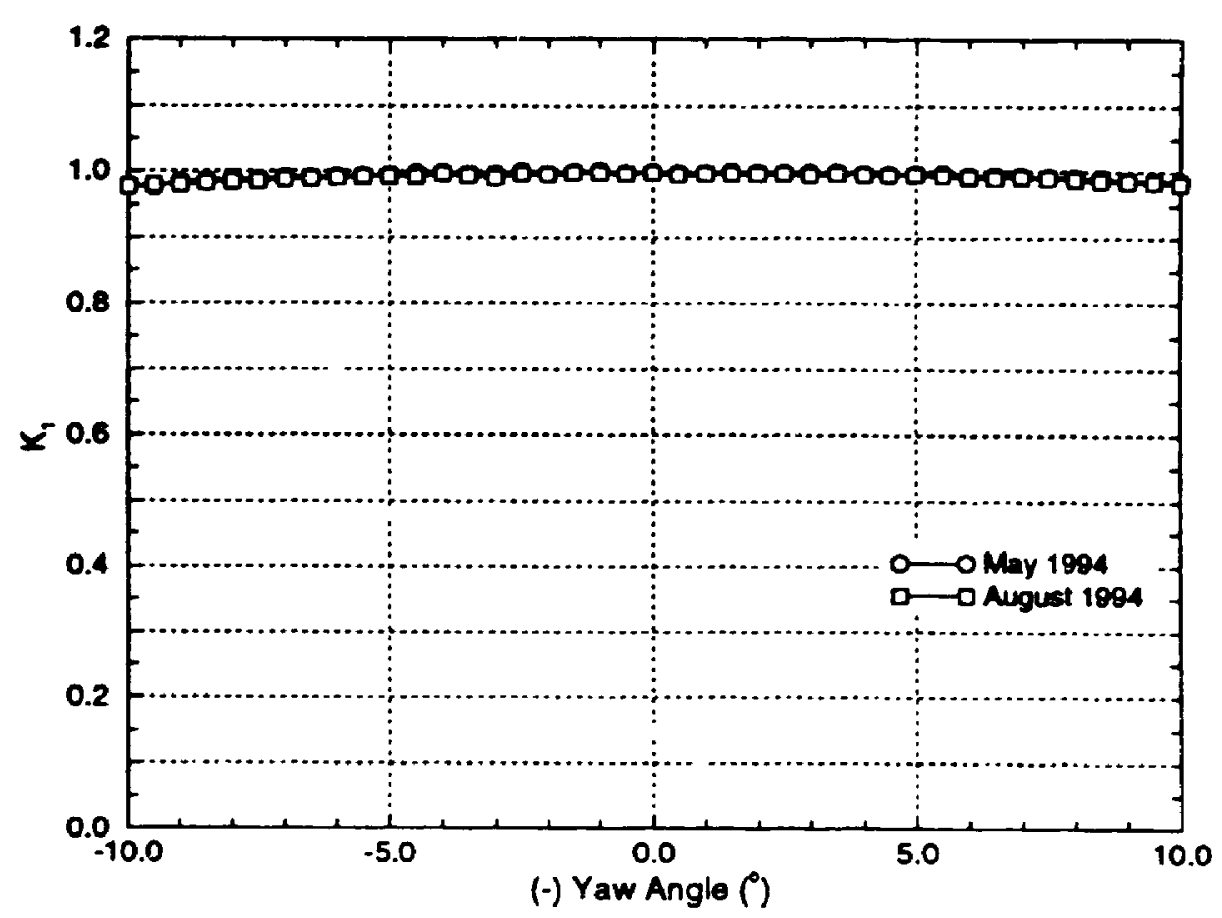

Figure C.2: Variation of $K_{1}$ with yaw angle for the outlet probe.

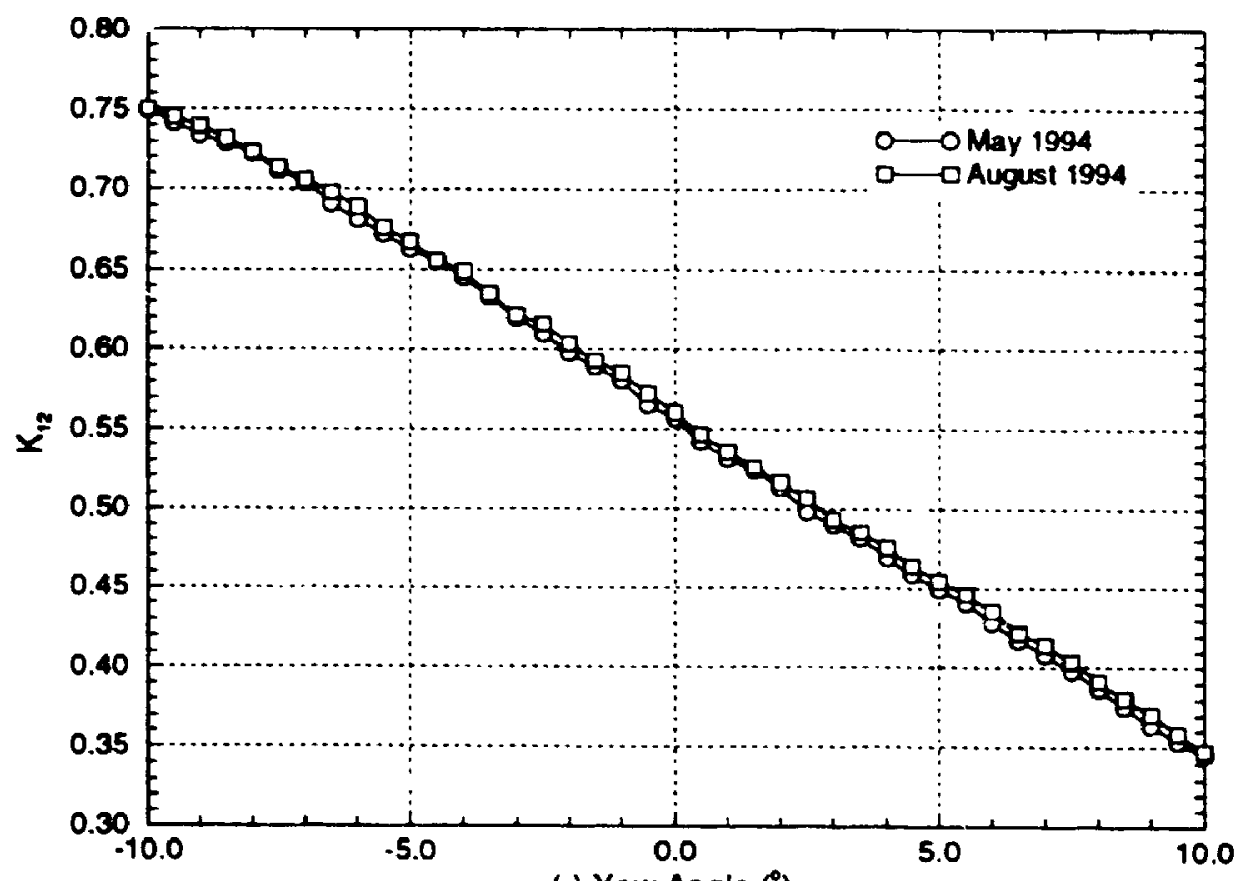

$(-)$ Yaw Angle ( $\left.{ }^{\circ}\right)$

Figure C.3: Variation of $K_{12}$ with yaw angle for the outlet probe. 


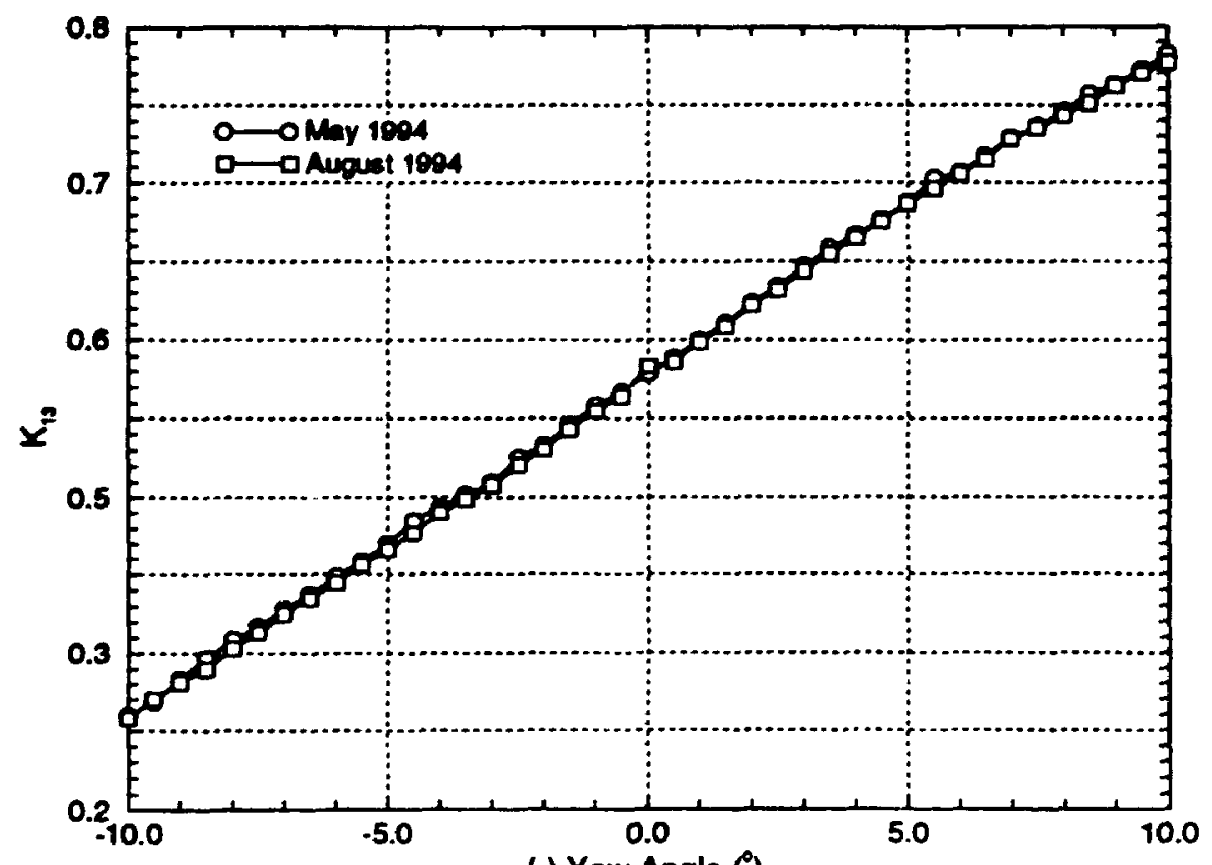

(-) Yaw Angle ()

Figure C.4: Variation of $K_{13}$ with yaw angle for the outlet probe.

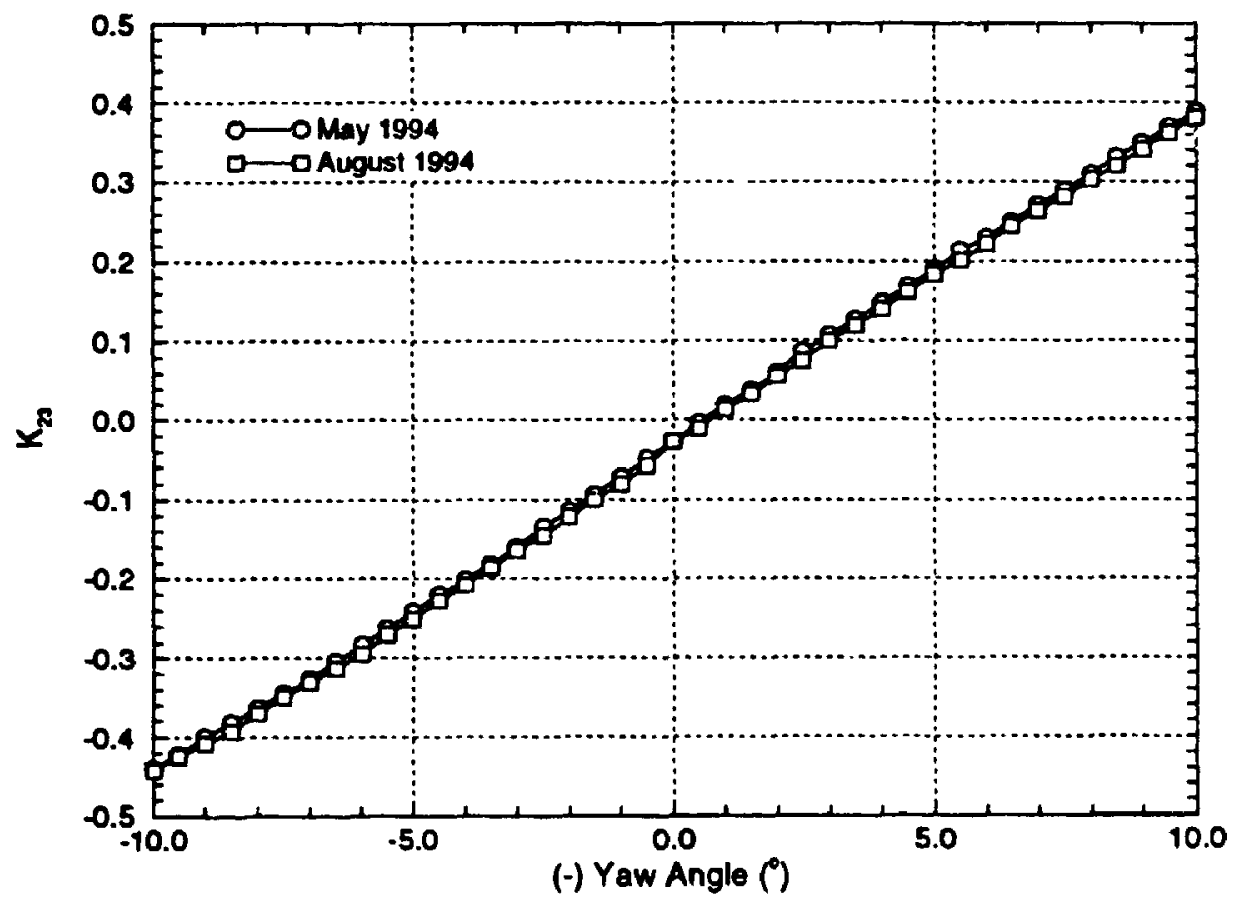

Figure C.5: Variation of $\mathbf{K}_{23}$ with yaw angle for the outlet probe. 


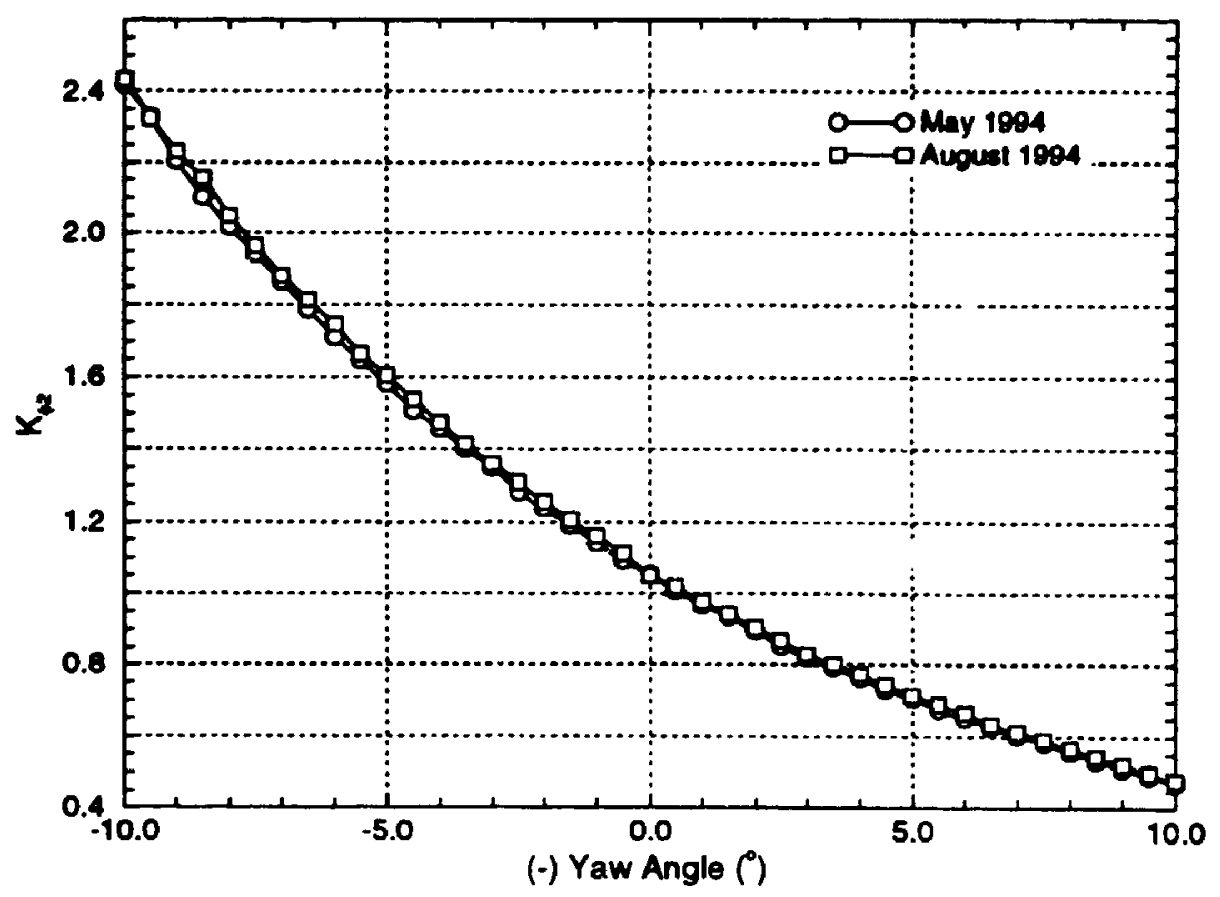

Figure C.6: Variation of $\mathrm{K}_{\phi 2}$ with yaw angle for the outlet probe.

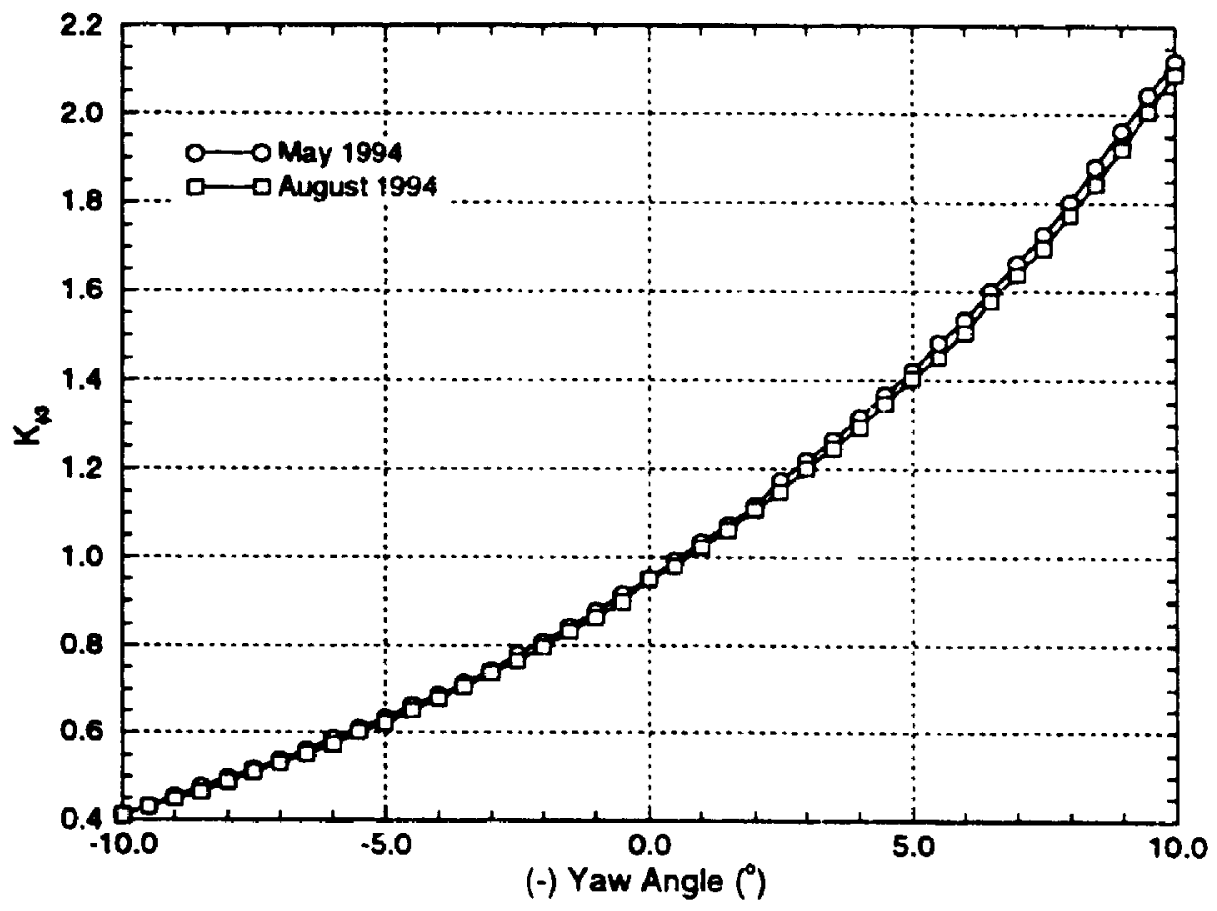

Figure C.7: Variation of $\mathrm{K}_{\phi 3}$ with yaw angle for the outlet probe. 
The calibration curves for both the Rosemount (Model 831 A3 serial \#567) and Baratron (Model 220DC serial \#79508-2) pressure transducers used in this research are presented in Figures C.8 and C.9. The calibration was performed using a micromanometer, a voltmeter and two $100 \mathrm{CC}$ syringes in parallel as a pressure source. The data presented in the figures below were obtained prior to beginning the experiments. Both pressure transducers were checked periodically during the research and found to be very stable.

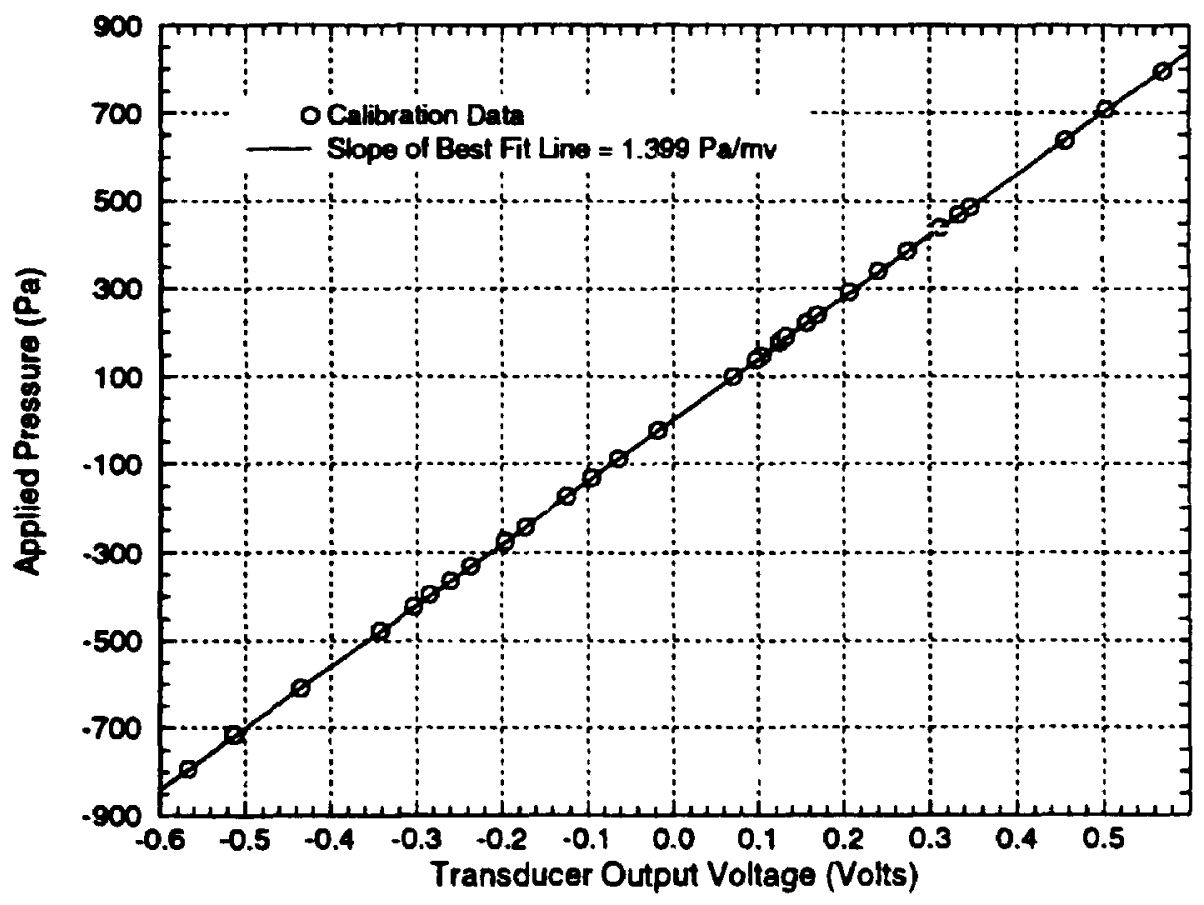

Figure C.8: Rosemount (Serial \#567) pressure transducer calibration curve. 


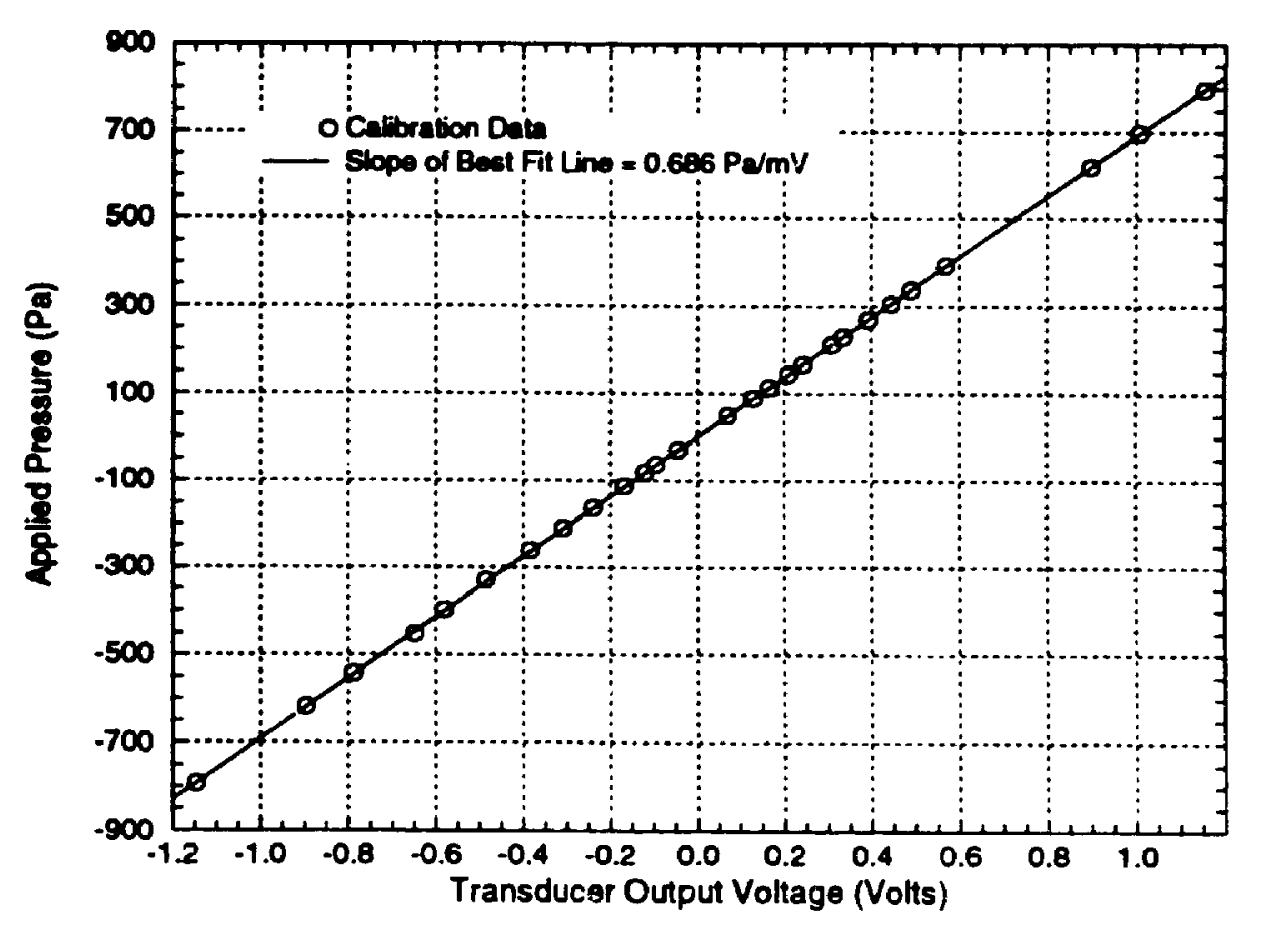

115

Figure C.9: Baratron (Serial \#79508-2) pressure transducer calibration curve. 


\section{Appendix D}

\section{Control Surface Settings}

This appendix tabulates the control surface and endplate initial settings used to establish a uniform, two dimensional flow in the test section for each angle of incidence, $i$, as indicated. A schematic diagram of the endplates and the test section control surfaces is given in Figures D.1 and D.2.

Table D.1: Control surface and endplate settings.

\begin{tabular}{|c|c|c|c|c|c|c|c|c|c|c|c|}
\hline \multirow{2}{*}{$\begin{array}{c}i \\
(9)\end{array}$} & \multicolumn{4}{|c|}{ Endplate Settings } & \multicolumn{7}{|c|}{ Control Surface Settings } \\
\hline & $\begin{array}{c}\mathbf{X 1} \\
(\mathrm{mm})\end{array}$ & $\begin{array}{c}\mathbf{X 2} \\
(\mathrm{mm})\end{array}$ & $\begin{array}{c}\mathbf{X 3} \\
(\mathrm{mm})\end{array}$ & $\begin{array}{c}X 4 \\
(\mathrm{~mm})\end{array}$ & $\begin{array}{l}\text { TF } \\
\text { (") }\end{array}$ & $\begin{array}{l}\mathbf{B F} \\
\text { ()) }\end{array}$ & $\begin{array}{l}\text { TTB } \\
\left({ }^{\circ}\right)\end{array}$ & $\begin{array}{c}\text { BTB } \\
\left(^{\circ}\right)\end{array}$ & $\begin{array}{c}\text { FW } \\
\left(^{\circ}\right)\end{array}$ & $\begin{array}{c}\text { BPI } \\
(\mathrm{mm})\end{array}$ & $\begin{array}{c}\text { BP2 } \\
(\mathrm{mm})\end{array}$ \\
\hline-10 & 32 & 141 & 32 & 139 & 23.0 & 22.5 & 68.0 & 70.0 & -2 & $\max$. & 0 \\
\hline 0 & 34 & 140 & 31 & 143 & 50.5 & 21.5 & 84.5 & 81.0 & -1 & 38 & 29 \\
\hline+10 & 31 & 141 & 31 & 143 & 56.5 & 40.5 & 97.0 & 91.0 & 0 & 50 & 41 \\
\hline+15 & 36 & 134 & 32 & 141 & 68.0 & 46.0 & 97.0 & 90.0 & 1 & 91 & 51 \\
\hline
\end{tabular}

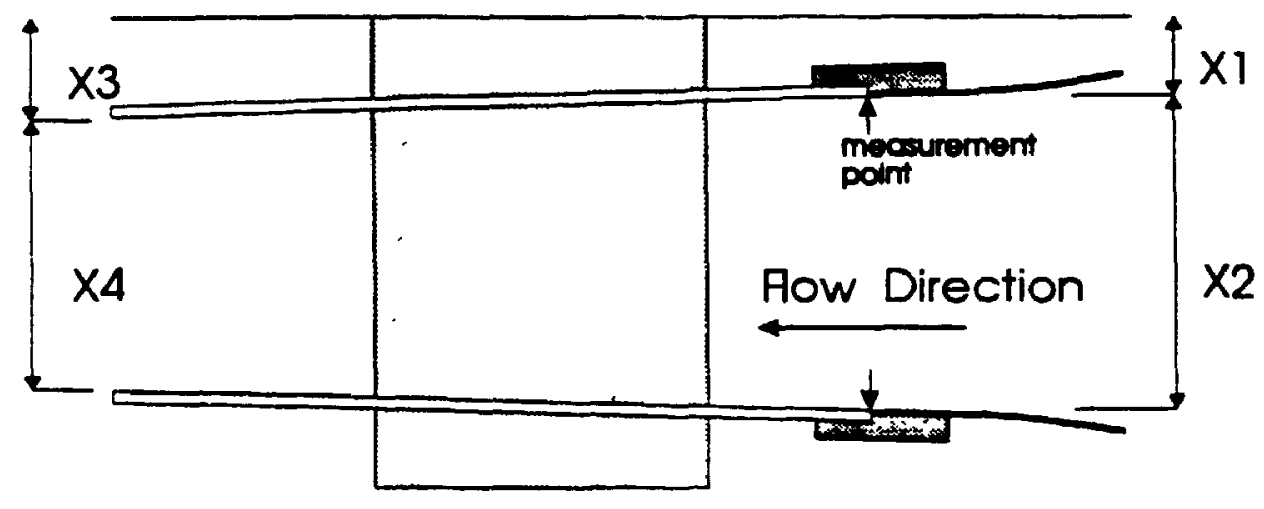

Figure D.1: Endplate nomenclature and settings - reproduced from Whitehouse (1993). 


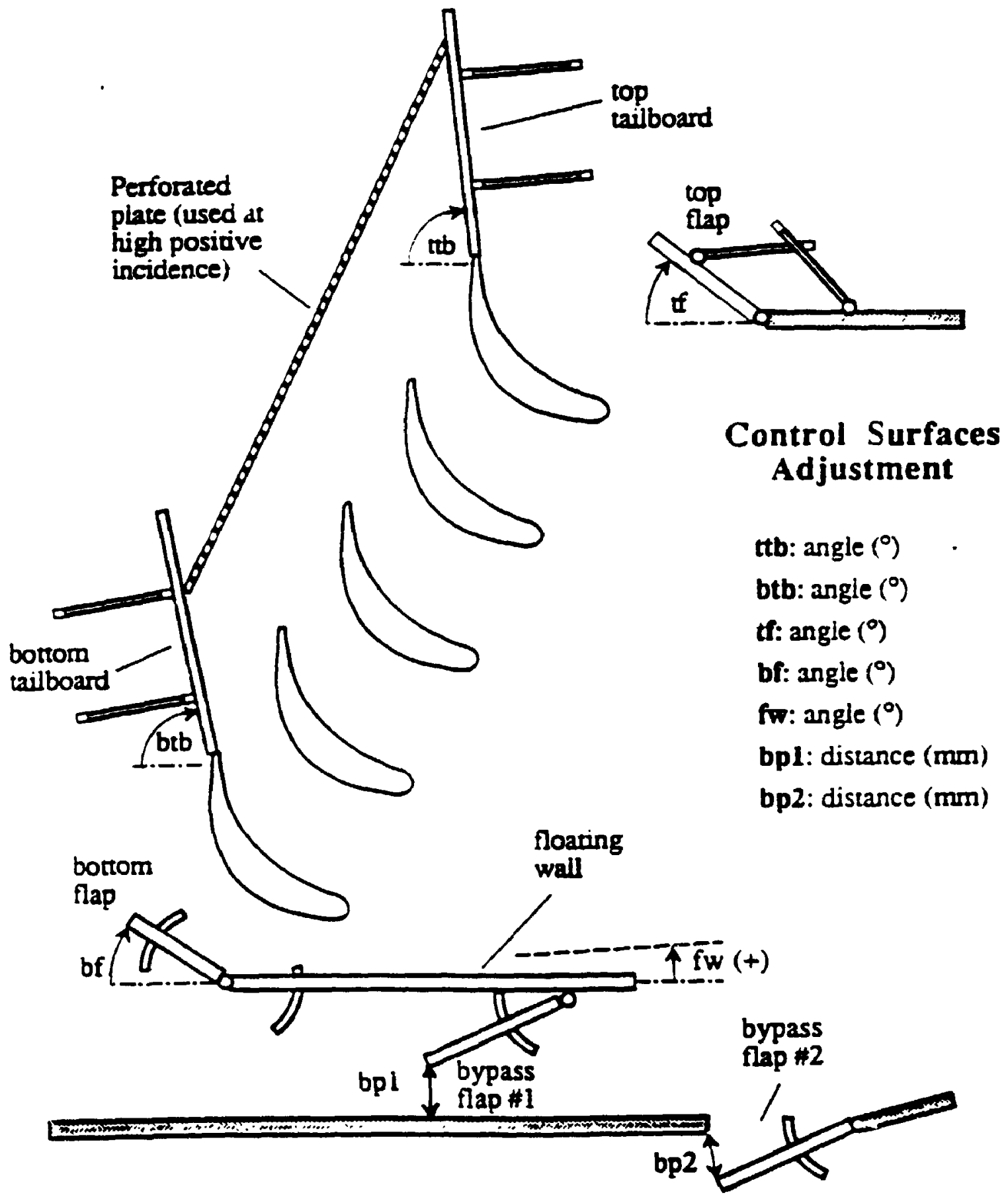

Figure D.2: Control surface locations and labelling - reproduced from Rodger (1992). 


\section{Appendix E}

\section{Spacing Parameter Calculation}

This appendix shows the calculation of the spacing parameter, $\lambda$, for each of the roughness configurations. The measurements were made by identifying the individual sand grains in a square arca of known size, $A_{r}$. Eight different areas were counted on three different blades and the average number of sand grains per blade was recorded (the approximate locations of the eight different areas are shown in Figure E.1). The average of all three blades was used to determine an average for the cascade. The frontal area, $A_{F}$, was calculated assuming that the grains were of spherical shape, and having a nominal diameter as determined by the mesh sizes of the sifting apparatus. Knowing the average particle size and the average number of particles per area, the spacing parameter was then easily determined using the relation:

$$
\lambda=\frac{A_{F}}{A_{T}}
$$


Table E.1: Spacing parameter calculation data.

\begin{tabular}{|c|c|c|c|c|c|c|c|c|c|c|c|c|c|c|}
\hline \multirow{2}{*}{$\begin{array}{c}\text { Trial } \\
\#\end{array}$} & \multirow{2}{*}{$\begin{array}{c}\text { Blade } \\
\qquad\end{array}$} & \multicolumn{8}{|c|}{ Measurements } & \multirow{2}{*}{$\begin{array}{l}\text { Blade } \\
\text { Ave.. }\end{array}$} & \multirow{2}{*}{$\begin{array}{c}\text { Cascade } \\
\text { Ave. }\end{array}$} & \multirow{2}{*}{$\begin{array}{c}A_{T} \\
\left(\mathrm{~cm}^{2}\right)\end{array}$} & \multirow{2}{*}{$\begin{array}{l}\text { Grain } \\
\text { Size } \\
(\mu \mathrm{m})\end{array}$} & \multirow{2}{*}{$\lambda$} \\
\hline & & 1 & 2 & 3 & 4 & 5 & 6 & 7 & 8 & & & & & \\
\hline \multirow{3}{*}{13} & 2 & 129 & 99 & 95 & 110 & 91 & 87 & 87 & 101 & 100.0 & \multirow{3}{*}{100.5} & \multirow{3}{*}{1} & \multirow{3}{*}{337.5} & \multirow{3}{*}{11.1} \\
\hline & 3 & 93 & 103 & 103 & 113 & 104 & 91 & 108 & 96 & 101.4 & & & & \\
\hline & 4 & 96 & 92 & 111 & 93 & 94 & 107 & 95 & 111 & 100.1 & & & & \\
\hline \multirow{3}{*}{$\begin{array}{c}9 \\
\& \\
10\end{array}$} & 2 & 77 & 87 & 86 & 102 & 98 & 126 & 134 & 121 & 103.9 & \multirow{3}{*}{107.6} & \multirow{3}{*}{9} & \multirow{3}{*}{1020} & \multirow{3}{*}{10.2} \\
\hline & 3 & 105 & 96 & 98 & 108 & 129 & 132 & 127 & 120 & 114.4 & & & & \\
\hline & 4 & 70 & T7 & 85 & 122 & 82 & 131 & 123 & 135 & 104.4 & & & & \\
\hline \multirow{3}{*}{$\begin{array}{c}14 \\
\& \\
15\end{array}$} & 2 & 69 & 89 & 72 & 80 & 69 & 100 & 112 & 111 & 87.8 & \multirow{3}{*}{88.9} & \multirow{3}{*}{4} & \multirow{3}{*}{722} & \multirow{3}{*}{11.0} \\
\hline & 3 & 70 & 83 & 86 & 92 & 85 & 78 & 102 & 79 & 84.4 & & & & \\
\hline & 4 & 92 & 71 & 83 & 105 & 90 & 115 & 110 & 89 & 94.4 & & & & \\
\hline \multirow{3}{*}{16} & 2 & 109 & 94 & 98 & 107 & 104 & 88 & 92 & 96 & 98.5 & \multirow{3}{*}{97.9} & \multirow{3}{*}{4} & \multirow{3}{*}{722} & \multirow{3}{*}{10.0} \\
\hline & 3 & 114 & 91 & 108 & 103 & 96 & 85 & 89 & 105 & 98.9 & & & & \\
\hline & 4 & 98 & 83 & 104 & 92 & 110 & 87 & 94 & 102 & 96.3 & & & & \\
\hline \multirow{3}{*}{17} & 2 & 101 & 94 & 97 & 105 & 115 & 121 & 99 & 111 & 105.4 & \multirow{3}{*}{104.9} & \multirow{3}{*}{9} & \multirow{3}{*}{1020} & \multirow{3}{*}{10.5} \\
\hline & 3 & 110 & 98 & 82 & 108 & 98 & 87 & 102 & 106 & 98.9 & & & & \\
\hline & 4 & 116 & 105 & 91 & 109 & 95 & 124 & 126 & 118 & 110.5 & & & & \\
\hline
\end{tabular}

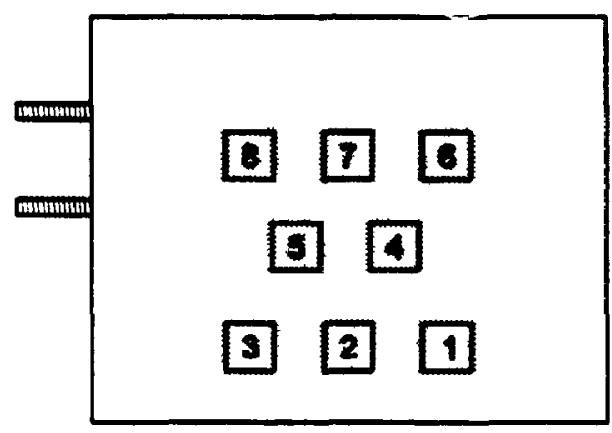

Proseure Surface

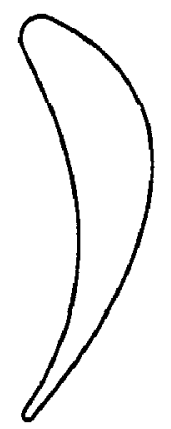

(8) 6 (5) 4 (3) 2

Suction Surface

Figure E.1: Approximate locations of the eight areas investigated for suction- and pressure- surface roughness samples. 


\section{Appendix $\mathrm{F}$}

\section{Three-Hole Probe Drawings}

All dimensions in millimeters $(\mathrm{mm})$.

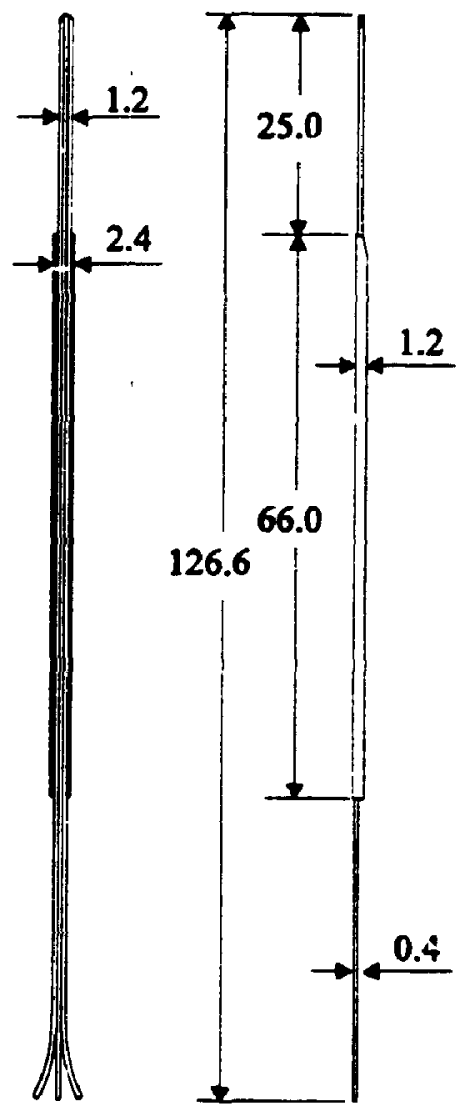

New Outlet Probe Design

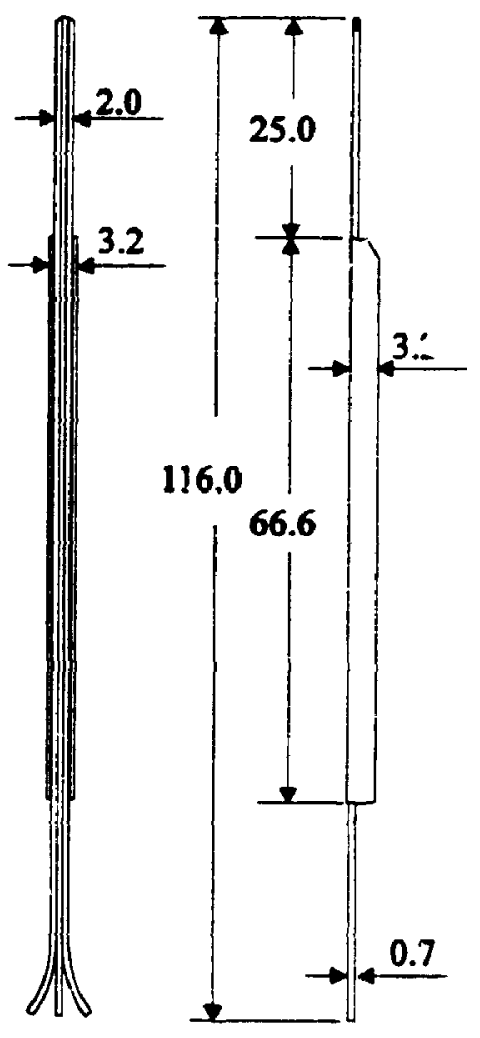

Outlet Probe Used in the Present Study

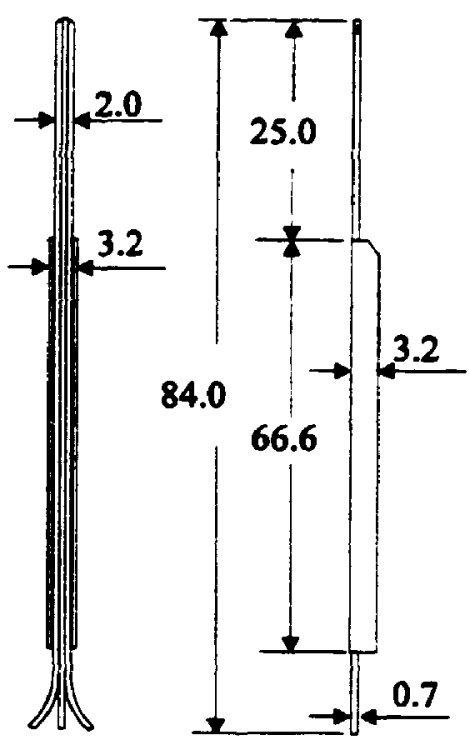

Inlet Probe

Figure F.1: Three-hole probes. 


\section{Appendix G}

\section{Sample Input File of the Modified Blade Geometry}

This appendix lists a sample input file for the modified blade profile used in the computational analysis. The first column lists the $x / c_{x}$ junction point coordinates and the second column lists the corresponding $y / c_{x}$ junction point coordinates. The listed coordinates start at the trailing edge and proceed counter-clockwise around the blade profile ending at the trailing edge. The last line lisis the separation point on both the suction- and pressure-surfaces (i.e. for the list shown the separation points are at the tr.iling e $: y)$.

\begin{tabular}{ll}
91 \\
1.000000 & 0.000000 \\
0.988483 & 0.024105 \\
0.984265 & 0.032400 \\
0.979007 & 0.042550 \\
0.973749 & 0.052500 \\
0.964866 & 0.068930 \\
0.953983 & 0.088600 \\
0.934218 & 0.123207 \\
0.914453 & 0.156200 \\
0.884806 & 0.202932 \\
0.855158 & 0.246429 \\
0.815629 & 0.299494 \\
0.776098 & 0.347635 \\
0.736567 & 0.390891 \\
0.697038 & 0.429309 \\
0.657507 & 0.463077 \\
\hline 0.617978 & 0.492319
\end{tabular}




$$
\begin{aligned}
& 0.5784470 .517329 \\
& 0.5389180 .538241 \\
& 0.4993870 .555234 \\
& 0.459858 \quad 0.568494 \\
& 0.4203270 .578196 \\
& 0.3807980 .584496 \\
& 0.341268 \quad 0.587488 \\
& 0.3017380 .587201 \\
& 0.2622070 .583615 \\
& 0.2325590 .578722 \\
& 0.2029110 .571853 \\
& 0.1831470 .566121 \\
& 0.1633820 .559419 \\
& 0.1436170 .551665 \\
& 0.1238510 .542773 \\
& 0.1040870 .532641 \\
& 0.0843220 .521081 \\
& 0.0645570 .507861 \\
& 0.0497330 .496691 \\
& 0.0398540 .488: 13 \\
& 0.0299680 .479641 \\
& 0.022557 \quad 0.472476 \\
& 0.0151440 .464825 \\
& 0.0083470 .455950 \\
& 0.0034410 .445902 \\
& 0.0006220 .435083 \\
& \begin{array}{lll}
0.000000 & 0.423922
\end{array} \\
& 0.0015990 .412856 \\
& 0.005357 \quad 0.402326 \\
& \begin{array}{lll}
0.011125 & 0.392749
\end{array} \\
& 0.0186740 .384503 \\
& \begin{array}{lll}
0.027707 & 0.377914
\end{array} \\
& 0.0378640 .373243 \\
& 0.0487460 .370676 \\
& 0.0599210 .370314 \\
& 0.0668180 .370772 \\
& 0.073717 \quad 0.371213 \\
& 0.0829140 .371781
\end{aligned}
$$




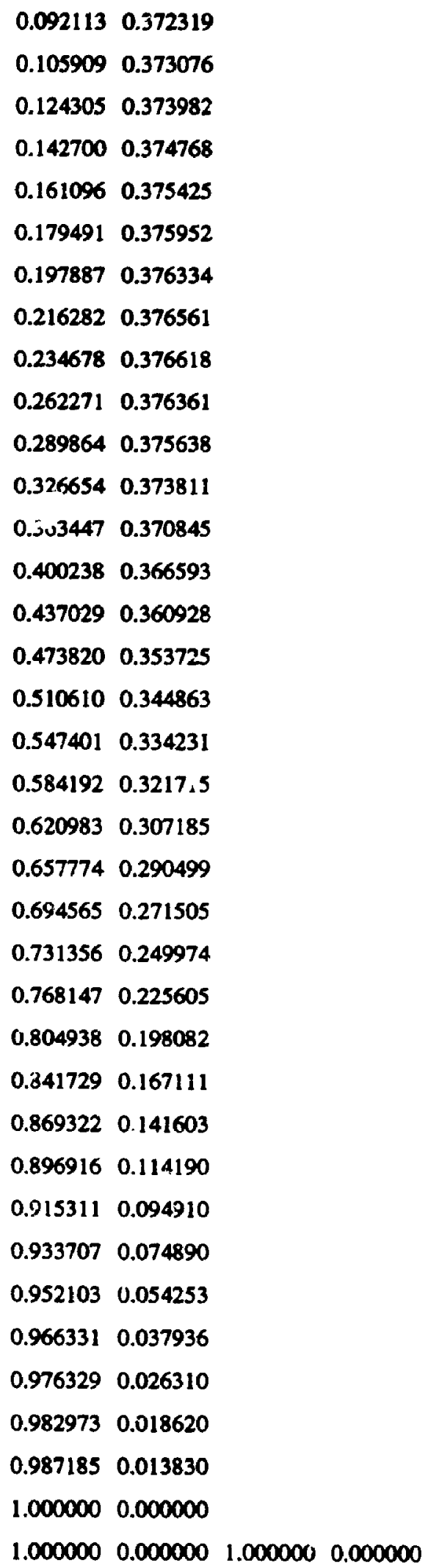




\section{Appendix $\mathbf{H}$}

\section{Derivation of the Computational Loss Coefificient}

This appendix presents a detailed derivation of the loss coefficient used in the computational model.

Consider a cross section of the wake, shown in Figure H.2, far enough downstream of the cascade that the static pressure and flow angle are uniform (i.e. at station 2):

(1)

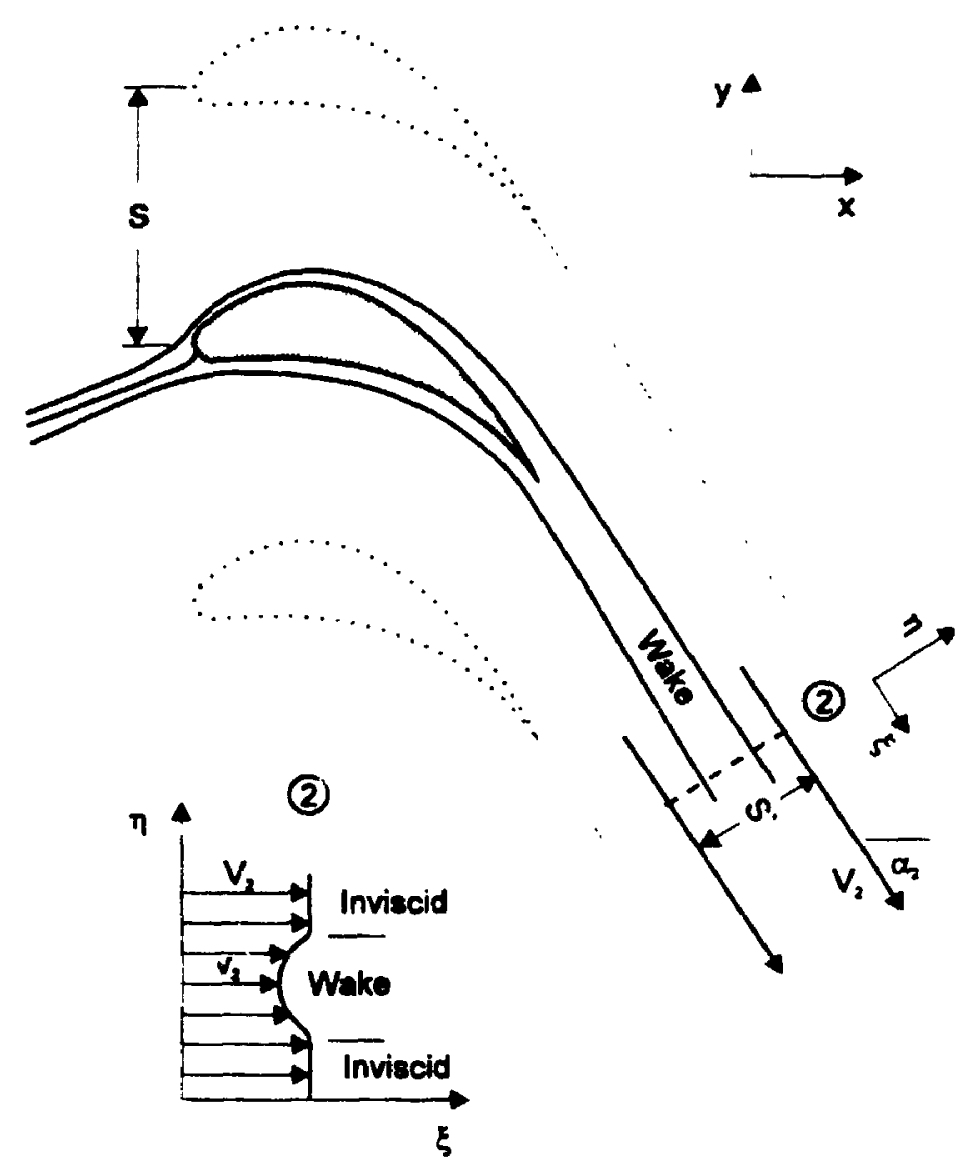

Figure H.2: Cross section of blade wake far downstream. 
where $V_{2}$ is the inviscid velocity and $v_{2}$ is the velocity in the wake.

The velocity distribution at station (2) in the $\eta$ direction is shown at the bottom of Figure H.2.

$$
\Delta P_{0} \equiv \frac{\int_{s^{\prime}}\left(P_{01}-P_{02}\right) d \dot{m}}{\int_{s} d \dot{m}}
$$

where $\int_{S^{\prime}}$ denotes integration in the $\eta$ direction over one space and $\dot{m}$ denotes the mass flow rate. Since $P_{01}=P_{2}+1 / 2 \rho V_{2}^{2}$ by Bernouilli and $P_{02}=P_{2}+1 / 2 \rho v_{2}^{2}$ by definition for any streamtube. Assuming the velocity at station (2) equals $v_{2}$ in general the above equation can be rewritten as:

$$
\begin{aligned}
& \Delta P_{0}=\frac{\int_{S} \rho v_{2}\left(\frac{1}{2} \rho V_{2}^{2}-\frac{1}{2} \rho v_{2}^{2}\right) d \eta}{\int_{S} \rho v_{2} d \eta} \\
& \Delta P_{0}=\frac{\frac{1}{2} \rho V_{2}^{3} \int_{S} \frac{v_{2}}{V_{2}}\left(1+\frac{V_{2}}{V_{2}}\right)\left(1-\frac{v_{2}}{V_{2}}\right) d \eta}{V_{2}\left(S^{\prime}-\delta_{n}^{*}\right)}
\end{aligned}
$$

where $\delta_{n}{ }^{*}$ is the displacement thickness of the wake. In the far wake, $\left(V_{2}-v_{2}\right)$ is small, therefore $v_{2} / V_{2} \approx 1$ and the approximation that $\left(1+v_{2} / V_{2}\right)=2$ could be made. giving.

$$
\Delta P_{0}=\frac{\frac{1}{2} \rho V_{2}^{2}}{\left(S^{\prime}-\delta_{*}^{*}\right)} 2 \theta_{\text {wahe }}
$$
where $\theta_{\text {wake }} \equiv \int_{S} \frac{v_{2}}{V_{2}}\left(1-\frac{v_{2}}{V_{2}}\right) d \eta$ is the momentum-tefect thickness of the wake. If
$\delta_{n} \cdot<S^{\prime}$ is assumed then, 


$$
\frac{\Delta P_{0}}{\frac{1}{2} \rho V_{2}^{2}}=\frac{2 \theta_{\text {wake }}}{S^{\prime}}
$$

since $S^{\prime}-S \cos \alpha_{2}$,

$$
\frac{\Delta P_{0}}{\frac{1}{2} \rho V_{2}^{2}}=\frac{2 \theta_{\text {wake }}}{S \cos \alpha_{2}}
$$

Therefore, if we can determine $\theta_{\text {wake }}$ and $\alpha_{2}$ from the computational analysis we can determine the loss coefficient based on the outlet conditions. The momentum thickness on the upper and lower surfaces, $\theta_{s s}$ and $\theta_{i}$ - respectively, are given from the boundary layer analysis. The problem is $\theta_{\text {wate }} \neq\left(\theta_{s S}+\theta_{P S}\right)$ unless the velocity at the trailing edge equals the velocity far downstream (ie. $U_{t e}=U_{\text {far }}$ ).

Between the trailing edge and far downstream the wake must obey the integralmomentum equation, with $c_{j}=0$ since there is no solid surface:

$$
\left.\frac{d \theta}{d x}\right|_{\text {wake }} ^{\text {plong }}+\frac{\theta}{U} \frac{d U}{d x}(H+2)=0
$$

This can only be solved if we have a relation between $H$ and $U$. Squire and Young (1938) used an empirical relation based on data for airfoils at small angles of attack. Since we don't have any corresponding data for cascades, and since we'd expect a cascade blade wakt to develop in essentially the same way as the wake of an isolated airfoil we will use the same result due to Squire and Young:

$$
\theta_{\text {wake }}=\left(\theta_{S S}+\theta_{P S}\right)\left(\frac{U_{k}}{U_{f a r}}\right)^{3.2}
$$


where $U_{f a r}=V_{2}=\frac{V_{1} \cos \alpha_{1}}{\cos \alpha_{2}}$ by continuity, neglscting the displacement effect of the wake. 

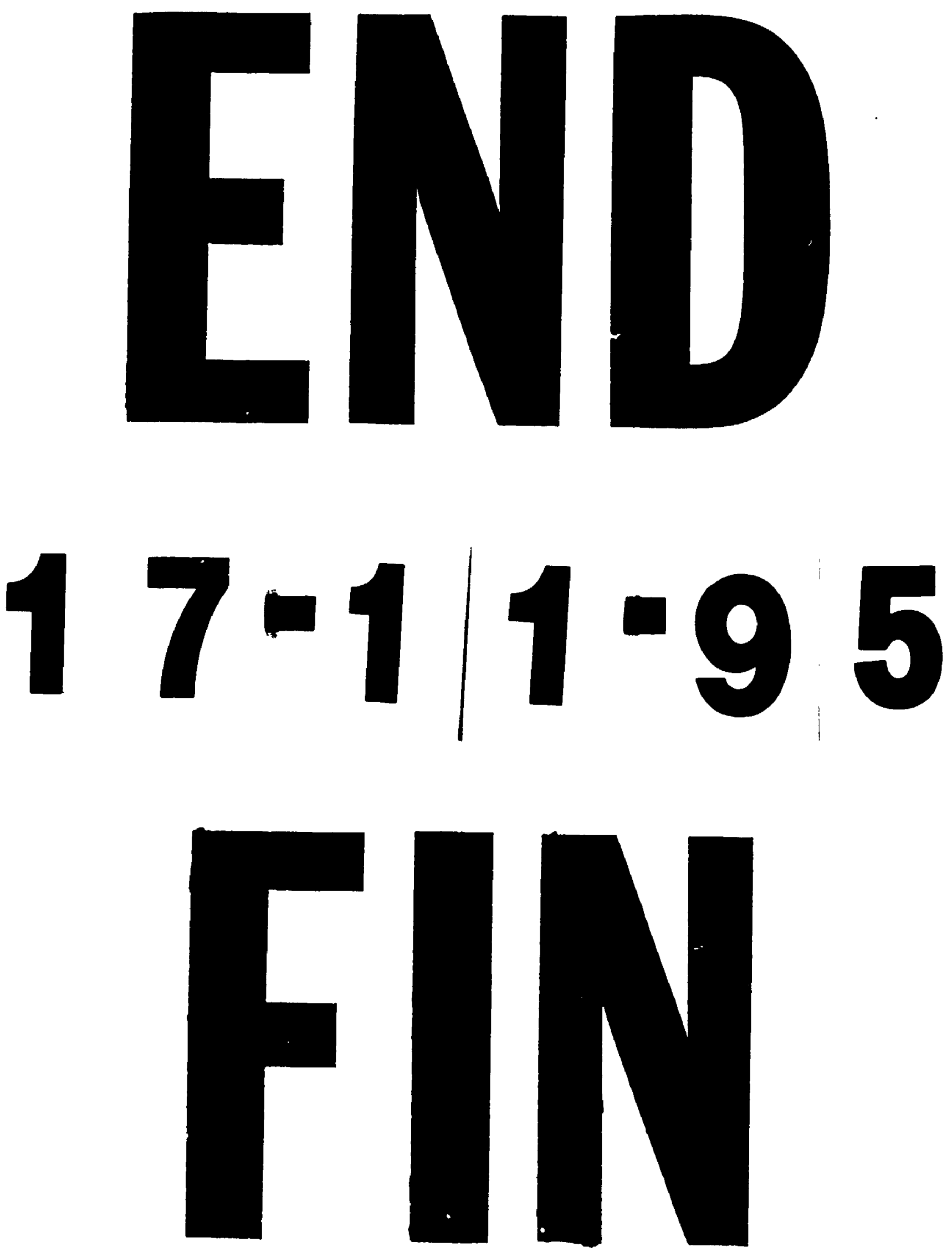